\title{
Understanding Phonon Interactions with Defects in Functional Oxide Materials
}

\author{
A Dissertation \\ Presented to \\ the faculty of the School of Engineering and Applied Science \\ University of Virginia \\ in partial fulfillment \\ of the requirements for the degree \\ Doctor of Philosophy \\ by \\ Brian F. Donovan
}

August

2016 


\section{APPROVAL SHEET}

The dissertation

is submitted in partial fulfillment of the requirements

for the degree of

Doctor of Philosophy

Author

The dissertation has been read and approved by the examining committee:

Prof. Pamela M. Norris, chair

Prof. Patrick E. Hopkins, advisor

Prof. Jerrold A. Floro

Prof. Joe Poon

Prof. Elizabeth C. Dickey

Prof. Bryan Kaehr

Accepted for the School of Engineering and Applied Science:

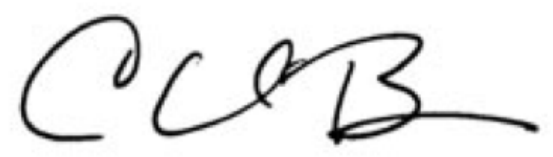

Craig H. Benson, Dean, School of Engineering and Applied Science

August

2016 


\section{Abstract}

Understanding of nano-scale thermal transport has enabled significant advances in a wide variety of fields including microelectronics, alternative energy, optics, and many more [1-4]. Utilized in many of these applications are a class of materials known as functional oxides. These materials are oxygen compounds with specific functional properties of interest (e.g., dielectrics [5-8], piezoelectrics [9-11], photovoltaics [12-14], thermoelectrics [15-19]). Often it is the defects in these materials that enable functionality and, depending on the processing and operating conditions, the concentration and types of defects that are present in these materials can vary widely [20]. In this dissertation, I aim to identify the role that defects play in functional oxide materials with respect to thermal transport.

The major thermal carriers in most functional oxide crystals are collective lattice vibrations, or phonons [21]. Phonon-dominated thermal conductivity is dictated by the scattering of these thermal carriers with a variety of other components or the material system, including defects in the crystal $[22,23]$. I concentrate on three types of defects that are common in functional oxides: boundaries, extrinsic point defects, and intrinsic point defects.

Boundaries such as film boundaries and grain boundaries with nano-scale dimensions can be detrimental to the thermal conductivity of a material. As the length of the uninterrupted crystal reduces to the phonon mean free paths in the crystal, phonons will scatter readily off of nano-scale boundaries and will not be able to propagate as they would in a bulk environment. This becomes particularly relevant in technologies such as the microelectronics industry, where device size scales are well below common phonon mean free paths in the constituent materials. To study this, I turn to one of the more common functional oxides used in the microelectronics industry, $\mathrm{BaTiO}_{3}$.

I study the effects of nano-scale grains on thin films of $\mathrm{BaTiO}_{3}$ grown via chemical solution deposition. The films studied are $150 \mathrm{~nm}$ and range in grain size from $36 \mathrm{~nm}-63 \mathrm{~nm}$. I show that the thermal conductivity of these nano-grained films scales with the grain size and film thickness and demonstrate agreement of this trend with analytical models. This result demonstrates that despite a complex crystal structure, there is a mean free path spectrum of phonons in $\mathrm{BaTiO}_{3}$ that significantly exceeds the dimensions of the grains and film (contrary to the common "gray" mean 
free path assumption seen in literature).

To address the role of extrinsic point defects, or dopants, on the thermal conductivity of functional oxides, I study the effects of dysprosium doping in thin films of cadmium oxide deposited by molecular beam epitaxy. In this experiment, the addition of Dy dopants in $\mathrm{CdO}$ actually increases the thermal conductivity initially, owing to a reduction in the equilibrium concentration of oxygen vacancies (a type of defect that is intrinsic to all oxides). The defect profile, and thus the thermal conductivity, can be further manipulated with more doping to subsequently decrease the phonon thermal conductivity, then increase the electronic thermal conductivity, and then ultimately saturate the system and scatter all thermal carriers leading to a two peak behavior in the thermal conductivity vs. Dy concentration curve. I isolate the role of various thermal carriers with measurements at $80 \mathrm{~K}$ as well as room temperature. I further explore the influence of this complex interplay of point defects with analytical modeling of the trends in phonon thermal conductivity, a process which reveals the signature of defect compensation which was not initially expected.

Change in intrinsic defect concentration in functional oxides can occur both from processing as well as defect evolution while under operation. During prolonged operation under high electric fields, defects in dielectric materials can undergo migration and pile up at the boundaries of the material. This phenomenon of dielectric degradation, which can lead to dielectric breakdown, is a major failure mechanism in electronic components. The buildup of heat can significantly accelerate this process, thus understanding the effects of increased concentration of intrinsic defects on thermal transport in functional oxides is critical.

I address this aspect of phonon-defect interaction in this dissertation with single crystals of rutile $\mathrm{TiO}_{2}$ that have been annealed in controlled oxygen environments to manipulate the intrinsic defect profiles. Using this processing technique, along with an analytical model for the thermal conductivity, I determine the effect that each type of intrinsic defect has on the thermal conductivity of the material. The results of this study show that the thermal conductivity of the $\mathrm{TiO}_{2}$ decreases significantly at defect concentrations exceeding 1 at.\%. As the defect concentration increases beyond 1 at.\%, the rutile crystal structure is eventually lost and new layered Magnéli phases form which have much lower thermal conductivity. This result will help to guide the understanding of the transport processes involved in dielectric breakdown, indicating a defect concentration threshold after which the impact to thermal transport becomes significant. In dielectric degradation, electro-migration 
can drive the formation a local high defect concentration layer on the size scale of $\sim 200 \mathrm{~nm}$, thus in addition to gaining insight into the thermal processes in general, the use of a nano-scale thermal measurement technique in this project can be directly carried over into extensions of this study.

I use time domain thermoreflectance (TDTR) to measure the thermal conductivity in each these experiments. TDTR is an ultra-fast optical pump-probe measurement technique that is ideal for the measurement of nano-scale thermal transport [24-26]. As each of the experiments described above has a component of nano-scale dimensionality (in the case of the $\mathrm{TiO}_{2}$, the extension of the experiment involves nano-scale defected layers), TDTR is an invaluable tool in the pursuit of understanding the effects of defects on thermal transport in these functional oxides.

The results in each of the studies discussed will provide valuable insight into the role of thermal transport in their respective applications. Furthermore, these results demonstrate a framework for understanding the impact defects on thermal transport in functional oxides and will lead to an advanced control of the thermal conductivity of oxides in general. 


\section{Acknowledgements}

I am lucky to have been able to collaborate with each member of my advisory committee, not only as advisors and mentors, but on other projects as well. I have learned a lot from the regular conference calls and discussions with Dean Norris. Professor Floro has been instrumental in challenging me to not only excel as a material scientist, but to concentrate on being an effective educator as well; something that I will take very seriously as I go on to teaching at the Naval Academy. The work that I have done with Professor Poon has been quite helpful in opening my eyes to the interdisciplinary nature of materials physics. Working with Dr. Kaehr, exploring measurement techniques, cross-phase heat transfer, and generally brainstorming in a comfortable intellectual environment has been a great experience. Professor Dickey has not only been a strong collaborator, but also mentor and friend. Working with Professor Dickey, I have developed my presentation and communication skills, solidified my materials science foundation, and gained experience writing a collaborative research proposal. I'd also like to acknowledge the community of scientists working on advancements in functional oxides including Jon Ihlefeld, Geoff Brennecka, Jon-Paul Maria, Clive Randall, Beth Dickey, Ed Gorzkowski, and many more.

The Exsite Lab has truly been a family to me during my time in Charlottesville. I would have never had this opportunity without the help and encouragement of Brian Foley. Brian was instrumental to my start in the lab and is the major reason that I was able to hit the ground running and continue to be productive throughout my career. Ramez Cheaito's excellence and attention to detail when it comes to TDTR has always been an example and personal challenge. It was a proud moment as an experimentalist after I had set up a custom TDTT rig and upon inspection, Ramez gave his relatively rare stamp of approval, "Ahhh very nice". John Gaskins was as critical to my success in the lab as he is to the success of the lab as a whole. While John's help quite literally enabled me to make my measurements, his unfailing support and camaraderie helped carry me through difficult times of my $\mathrm{PhD}$. It is not often that you find a colleague that can make searching for a micrometer sized hole in a gold film an enjoyable activity. Ash Giri has been like a brother to me; a brother that can seamlessly move between in depth discussions of the nature of vibrational transport across multiphase interfaces to laughing at the simple comedy of solar powered sumo wrestler that never stops dancing on your desk. Ash's work ethic and productivity have served as a challenge to drive myself to achieve the most of my potential while never overstepping into contention. I have really enjoyed the new additions to the lab as well, whether brainstorming ideas with a fresh approach or helping solidify concepts by explanation, having Jeff, Chet, Kelsey, Mallory, and Lei in the lab has been a great experience.

The best thing that a graduate student can hope for is a good fit with their advisor. In my case, "good fit" is quite an understatement. Working with Patrick has not only led to a successful PhD experience, but I have grown substantially as a person, researcher, teacher, academic, and the list goes on. Brainstorming new thermal characterization methods and interesting thermophysical systems has been a particular pleasure. Patrick has done an amazing job of enabling me to take personal charge of my projects, instilling me with a sense of ownership, allowing me to explore while still guided, and importantly, enabling me to connect with 
collaborators to make a name for myself. Thanks to Patrick's encouragement and guidance, my writing and presentation skills have vastly improved over the past three years. This final year of my PhD, I have been lucky enough to teach alongside Patrick, and that has been an amazing experience. In Patrick I know I have found not only a mentor and future collaborator, but I have found a lifelong friend.

Of course, without my parents there is no way I would be in the position that I am today. My parents have instilled an unrelenting work ethic in me, a resolute sense of confidence, and a strong passion for my work and community. My dad taught me that if there is a task at hand, you put your head down and do it, regardless of whether you like it or not. While sometimes this can mean suffering through a chore, it often meant teaming up and finding the best way to not only solve the problem, but to enjoy doing it in the meantime. From writing college essays with my step-mom to fixing the car in the middle of the Wal-Mart parking lot, I cannot thank my parents enough for teaching me to embrace the situation that I am in and face it head on. My mother's unconditional love for her community and pursuit of education, both in learning and teaching, have always been an example for me. My mom and step-dad have shown me what it means to give everything you have to support those around you. My mother's passion for education has pushed me towards the place that I am in today, whether I like it or not. She has always pursued knowledge and, while she is proud of me for everything I do, has always been particularly proud of my accomplishments as a teacher and an academic. My brothers are a source of stability in my life. My older brother, Steven, has always been there to keep me grounded, to sympathize with any complaint, and to celebrate in any victory. Growing up, we were always together and (almost) always a team. He is a constant reminder to me of what it means to be proud of who you are and stand strong in whatever it is you are doing. My step-brother, Tommy, is an enduring source of support and love. No matter how long it has been since I have last seen him, or how far apart we are, I know I can always count on him to be the brother that he has been since we were 11 years old. I could not have asked for a better family foundation to have come from, and I hope that all of the members of my family from my parents, to my brothers, to my nieces, aunts, uncles, and grandparents, know how thankful I am for their support.

More recently, I have been lucky to add more immediate family to the list. My mother and father-in-law, Mary and Ken, and sister-in-law, Michelle, have graciously accepted me as one of their own and I am grateful for the fact that I truly feel like part of the family.

Most importantly, I must thank the center of my life, my wife Samantha. Coming home to Sam and our dogs, Zoey and Gibbs, every day over the past three years has been unbelievable. I often wonder what I did right in a past life to deserve the family that I have. Samantha has supported me intrinsically throughout everything that I have done, she encourages me to be the best person that I possibly can, to pursue everything that makes me happy, and has proudly stood by me along this journey. With Sam I can delve into complex material science problems or complain about monotonous assignments and know that I have a truly sympathetic shoulder to lean on. Sam provides me with perspective in life, a constant reminder of what is most important. If that isn't nice, what is? 
Ting-a-ling! 


\section{Table of Contents}

1 Introduction $\quad 11$

1.1 Phonon Defect Scattering . . . . . . . . . . . . . . . . . 13

1.2 Thermal Transport in Functional Oxides . . . . . . . . . . . . . . . . . . . . 15

1.3 Applications of Thermal Transport in Functional Oxides . . . . . . . . . . . . . . . . . 19

1.3.1 Functional Oxide Applications in Thermal Mitigation . . . . . . . . . . . . 19

1.3.2 Functional Oxide Applications in Thermal Insulation . . . . . . . . . . . . . . 20

1.3.3 Functional Oxide Applications in Thermal Tuning . . . . . . . . . . . . . . . 24

1.4 Statement of Objectives and Impact . . . . . . . . . . . . . . . . . . . . 27

2 Background $\quad 31$

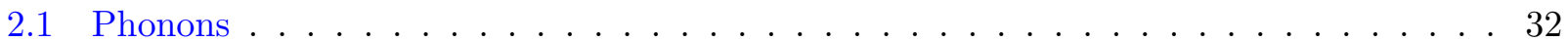

2.2 Modeling Phonon Transport . . . . . . . . . . . . . . . . . . 35

2.3 Formation of Point Defects in Oxides . . . . . . . . . . . . . . . . . . . 44

2.4 Interaction of Point Defects in Reduced $\mathrm{TiO}_{2} \ldots \ldots \ldots \ldots$. . . . . . . . . 53

2.5 Dielectric Breakdown . . . . . . . . . . . . . . . . . . . 58

3 Measurement of Nanoscale Thermal Transport $\quad 63$

3.1 Frequency Domain Thermoreflectance . . . . . . . . . . . . . . . 63

3.2 Time Domain Thermoreflectance . . . . . . . . . . . . . . . . 66

3.3 Thermal Transport in a Layered Structure with a Modulated Source . . . . . . . . . . 73

3.4 Analysis of Lock-in Detection of a Thermoreflectance Signal . . . . . . . . . . . . . . . 80

3.5 TDTR Sensitivity Analysis . . . . . . . . . . . . . . . . 87

4 Phonon Interactions with Grain Boundaries in $\mathrm{BaTiO}_{3} \quad 89$

4.1 Literature Thermal Conductivity of $\mathrm{BaTiO}_{3} \ldots \ldots \ldots \ldots$. . . . . . . . 90

$4.2 \mathrm{BaTiO}_{3}$ Film Fabrication and Characterization . . . . . . . . . . . . . . . 92

4.3 Thermal Measurements and Phonon-Grain Boundary Interactions . . . . . . . . . . 95 
5 Phonon Interactions with Extrinsically Controlled Point Defects in CdO

5.1 CdO Synthesis and Defect Control . . . . . . . . . . . . . . . . . . . . 104

5.2 Modeling Phonon-Point Defect Interactions in CdO . . . . . . . . . . . . 106

5.3 Experimental Evidence of Phonon-Point Defect Interactions . . . . . . . . . . . . 107

6 Phonon Interactions with Intrinsic Point Defects in $\mathrm{TiO}_{2} \quad 115$

6.1 Applications and Background of $\mathrm{TiO}_{2} \ldots \ldots \ldots \ldots$

6.2 Measurement and Control of Reduced $\mathrm{TiO}_{2} \ldots \ldots \ldots \ldots$. . . . . . . 117

6.3 Modeling Phonon Transport in Rutile $\mathrm{TiO}_{2} \ldots \ldots$. . . . . . . . . . . . . . 119

6.4 Impact of Defects on the Thermal Conductivity of Reduced $\mathrm{TiO}_{2} \ldots \ldots$. . . . . . 125

7 Summary $\quad 133$

7.1 Opportunities for Further Investigation _ . . . . . . . . . . . . . . . . . . 135

7.2 Contributions to the Scientific Body of Knowledge . . . . . . . . . . . . 138

7.3 Published Work . . . . . . . . . . . . . . . . . . . 139

\section{List of Figures}

1.1 Illustration of defect types . . . . . . . . . . . . . . . . . 12

1.2 Spectral thermal conductivity and defect interactions . . . . . . . . . . . . . 14

1.3 Rocksalt \& perovskite crystal structures . . . . . . . . . . . . . . 16

1.4 Thermal conductivity of complex oxide crystals . . . . . . . . . . . . 17

1.5 Thermal conductivity of thermal barrier coating materials . . . . . . . . . . . 21

1.6 Literature results of thermal conductivity tuning using non-stoichiometry in $\mathrm{SrTiO}_{3} \quad$. 25

1.7 Thermal conductivity tuning using coherent crossover in superlattices . . . . . . . . 26

2.1 Illustration of a phonon $\ldots \ldots \ldots \ldots \ldots \ldots$

2.2 Phonon dispersion diagrams for some common crystals . . . . . . . . . . . . . 34

2.3 Bose-Einstein Distribution . . . . . . . . . . . . . . . . . . 37

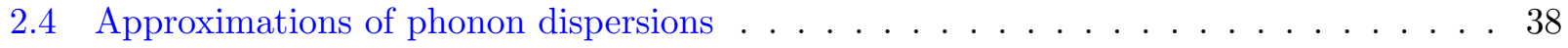

2.5 Thermal conductivity of $\mathrm{TiO}_{2}$ by phonon scattering regime . . . . . . . . . . . 43

2.6 Change in Gibbs free energy due to vacancy formation . . . . . . . . . . . . . 46 
2.7 Brouwer diagram for a general metal oxide . . . . . . . . . . . . . 51

2.8 Crystallographic shear planes in reduced $\mathrm{TiO}_{2} \quad \ldots \ldots \ldots \ldots \ldots$

2.9 Phase diagram for the titanium-oxygen . . . . . . . . . . . . . . . . 54

2.10 X-ray diffraction patterns of Magnéli phases . . . . . . . . . . . . . . 55

2.11 TEM Images of the Magnéli phase structure . . . . . . . . . . . . . . 56

2.12 Electron diffraction image of Magnéli phase . . . . . . . . . . . . . . . . 57

2.13 Dielectric breakdown imaging . . . . . . . . . . . . . . . . . . 59

2.14 Effects of processing on dielectric breakdown . . . . . . . . . . . . . . . 60

2.15 Example of dielectric breakdown measurement . . . . . . . . . . . . . 61

2.16 Schematic of the dielectric breakdown process $\mathrm{in}^{\mathrm{SiO}_{2}} \ldots \ldots \ldots$

3.1 Pulse-CW FDTR schematic . . . . . . . . . . . . . . . . . . 63

3.2 Thermal penetration depth vs. modulation frequency . . . . . . . . . . . . . 65

3.3 Typical TDTR scan . . . . . . . . . . . . . . . . 67

3.4 TDTR schematic . . . . . . . . . . . . . . . . . . . 68

3.5 Unit circle representing the in-phase and out-of-phase TDTR signals . . . . . . . . 72

3.6 Sensitivity to in-plane thermal conductivity of $\mathrm{BaTiO}_{3}$ films . . . . . . . . . . 75

3.7 Flowchart of thermoreflectance information . . . . . . . . . . . . . 80

3.8 Sensitivity of TDTR to Thermal Boundary Conductance \& Thermal Conductivity . . 87

4.1 XRD patterns of $\mathrm{BaTiO}_{3}$ films . . . . . . . . . . . . . . . . . . 94

4.2 SEM images of $\mathrm{BaTiO}_{3}$ films . . . . . . . . . . . . . . . . . . 95

4.3 AFM images of $\mathrm{BaTiO}_{3}$ films $\ldots \ldots \ldots \ldots \ldots \ldots$

4.4 Thermal conductivity measurements of nano-grained $\mathrm{BaTiO}_{3} \ldots \ldots \ldots$

4.5 Temperature dependent thermal conductivity of nano-grained $\mathrm{BaTiO}_{3} \ldots \ldots$

5.1 Transport characteristics with increasing Dy concentration in $\mathrm{CdO} \ldots$. . . . . . 105

5.2 Thermal conductivity of CdO:Dy at various concentrations of Dy . . . . . . . . 109

5.3 Phonon dispersion of $\mathrm{CdO} \ldots \ldots \ldots \ldots \ldots \ldots$

5.4 Temperature dependent thermal conductivity of undoped $\mathrm{CdO} \ldots$. . . . . . . . 112

5.5 Phonon thermal conductivity of CdO:Dy at various concentrations of Dy $\ldots 113$

6.1 Rutile unit cell . . . . . . . . . . . . . . . . . . . . 116

$6.2 \mathrm{TiO}_{2}$ Brouwer diagram . . . . . . . . . . . . . . . . . . . 119 
6.3 Phonon dispersion of $\mathrm{TiO}_{2} \ldots \ldots \ldots \ldots \ldots$

6.4 Model of impact of defects on the thermal conductivity of $\mathrm{TiO}_{2} \ldots \ldots$. . . . . . . 124

6.5 Contribution of types of intrinsic defects to the thermal resistivity of $\mathrm{TiO}_{2} \ldots \ldots$

6.6 Measured thermal conductivity of reduced $\mathrm{TiO}_{2} \ldots \ldots \ldots \ldots$

6.7 Sub-stoichiometry of $\mathrm{TiO}_{2}$ vs. $\mathrm{pO}_{2} \ldots \ldots \ldots \ldots \ldots \ldots$

6.8 X-ray diffraction patterns of defected $\mathrm{TiO}_{2} \ldots \ldots \ldots \ldots \ldots \ldots$

6.9 Electron diffraction pattern of $\mathrm{Ti}_{n} \mathrm{O}_{2-n} \ldots \ldots \ldots \ldots \ldots \ldots$

6.10 Additional electron diffraction pattern of $\mathrm{Ti}_{n} \mathrm{O}_{2-n} \ldots \ldots \ldots \ldots$

6.11 TEM image and electron diffraction patterns of the onset of layered defects in $\mathrm{TiO}_{2} \quad .130$

\section{List of Tables}

1 Phonon scattering factors . . . . . . . . . . . . . . . . . . 42

2 Functional form of the lock-in for various pump-probe schemes $\ldots \ldots \ldots 6$

$3 \quad$ Literature values of thermal conductivity of $\mathrm{BaTiO}_{3} \ldots \ldots \ldots \ldots 2$

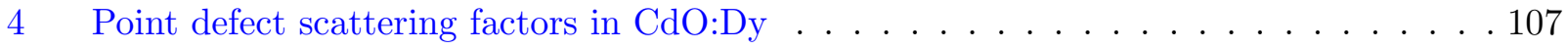




\section{$1 \quad$ Introduction}

Many recent developments in materials technology are centered around nanoscale applications which generate a significant amount of energy, such as integrated microelectronics, or capture generated energy, such as photovoltaics and thermoelectrics. In these applications, where size scales of technological dimensions and thermal carriers begin to intersect, thermal characterization becomes critically important. In this regime, nanometer sized defects can significantly change the way in which heat moves through a material. There are many different types of materials that are critical to applications where nanoscale thermal characterization is needed. In the case of microelectronics, we need to know the thermal properties of the metal interconnects, the semiconducting active layers, and the dielectric functional oxides, to name a few. For each of those components, understanding how changes in nanoscale properties affect thermal transport is crucial to enabling optimal performance.

In this dissertation, my goal is to determine how thermal transport is affected by defects in functional oxide materials. To accomplish this, we must understand what functional oxides are, be able to control the defects present in them, and be able to measure and understand the thermal transport properties in these defected materials of interest. I will present the necessary fundamental concepts and use representative experiments to build an understanding of the interaction between thermal carriers and defects in functional oxides.

Functional oxides, such as $\mathrm{TiO}_{2}$ or $\mathrm{BaTiO}_{3}$, are a class of materials that are oxygen compounds, often with metals, which have some intrinsic electronic or magnetic property of technological interest [27]. These properties can include semiconductivity [28, 29], ferroelectricity [30-32], ferromagnetism [29, 33-36], or superconductivity [37-39]. Functional oxides are used in applications including microelectronics [5, 8, 40-45], photovoltaics [12, 13, 46, 47], piezoelectrics [9-11], and thermoelectrics [16, 18, 19, 48-51]. I will discuss much more about the applications, and specifically the thermally driven aspects of the applications utilizing functional oxide materials below and in the chapters to come. In addition to the diversity in functionality and application, these materials have been fabricated in a slew of different methods and can be configured into geometries ranging from bulk single crystals to nanoparticles. This flexibility in processing techniques will allow us to dictate the parameters of our study in a way that isolates thermal transport properties and highlights the dynamics between 


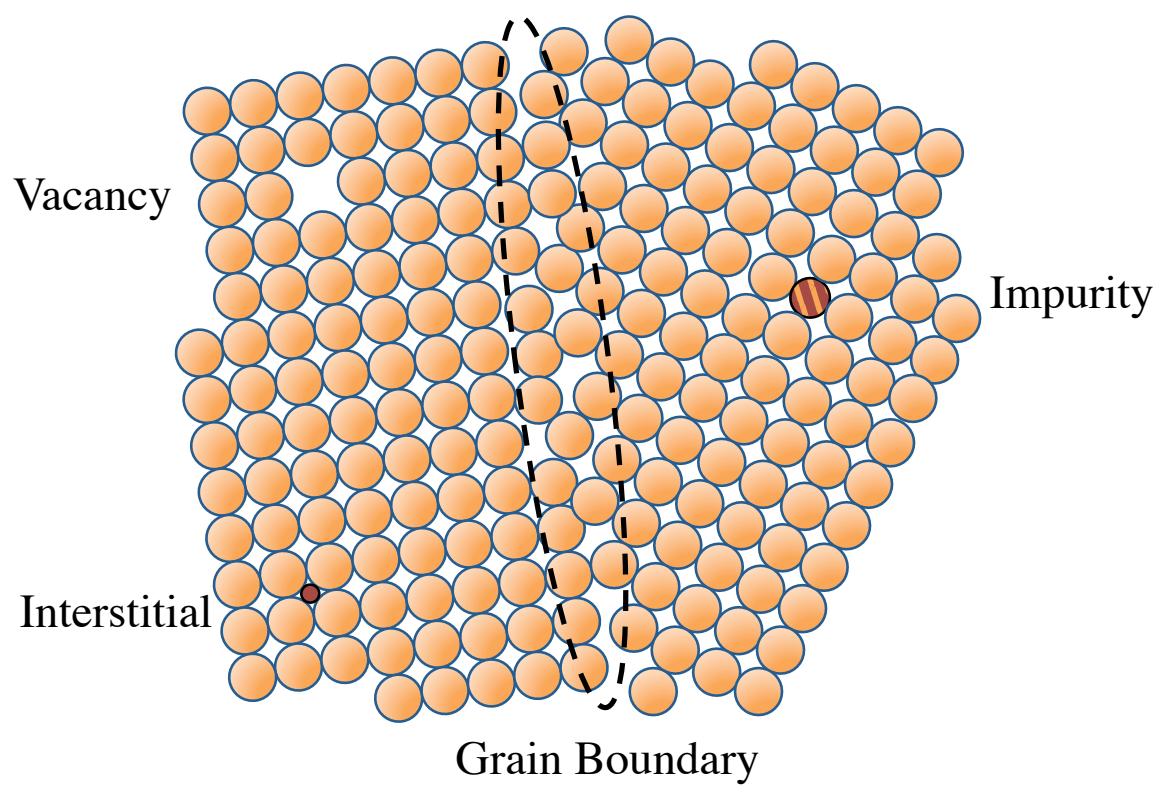

Figure 1.1: Illustration of major types of oxide crystal defects addressed in this dissertation, including grain boundaries, interstitial atoms, substitutional impurities, and vacancies.

thermal transport and defects.

All materials have defects $[52,53]$. The common types of microstructural defects in fully dense oxides can be generally separated into three major categories: boundaries, line defects, and point defects. Boundaries are essentially dictated by the degree of crystallinity, where a single crystal has boundaries defined by the sample and polycrystalline materials can have smaller grain or domain boundaries. In this dissertation, I will be working commonly with boundaries in materials that range from nanometers (with thin and nano-grained films) to millimeters (with bulk single crystals). Line defects are usually dislocations in crystalline materials, a topic which I will not cover in this dissertation. Lastly, there are point defects, which are extremely important in functional oxides. Point defects are atomic scale imperfections consisting of missing atoms (vacancies), the wrong types of atoms (substitutional defects), or atoms where there should not be atoms (interstitials). The presence of point defects in a crystal is thermodynamically driven, that is to say that any crystal in equilibrium will have point defects. In functional oxides, point defects can lead to functionality and dictate conducting properties via the transport of species such as oxygen vacancies or mobile cations. Additionally, the concentration of point defects in oxides is environmentally dependent, dictated by the amount of oxygen surrounding the material, which is critically important since we live in an oxygen rich atmosphere. 
The last piece of the puzzle to introduce is thermal transport. In crystalline materials, heat is largely transported in one of two ways, either by mobile electrons, or by vibrations of the atoms in the crystal $[21,22]$. If a material is electrically conductive, most of the heat is usually transported by electrons, since they are able to move significantly faster than the atomic vibrations can propagate. If this is not the case, the heat is carried by atomic vibrations. With a crystalline lattice and finite boundaries, atomic vibrations can only take on certain wavelengths and frequencies (the longest possible wavelength dictated by the crystal boundary and the shortest wavelength by the atomic spacing). We define the various modes of these vibrations, each with their given wavelength and frequency, as phonons. I will not go into extreme depth into the definition of a phonon, but it is important to have some idea of this heat-carrying quasiparticle, as phonons are the major thermal transport mechanism in many functional oxides.

In phonon-dominated thermal transport, we can define the thermal conductivity using an equation taken from the kinetic theory of gasses,

$$
\kappa=\frac{1}{3} C v l=\frac{1}{3} C v^{2} \tau
$$

where the thermal conductivity, $\kappa$, is equal to the product of the energy the thermal carrier can store, or the heat capacity, $C$, the velocity of the thermal carrier, $v$, and the distance the thermal carrier can travel before scattering given by the mean free path, $l$, or the scattering time, $\tau$.

This last component, the phonon mean free path, is the major aspect that is impacted by defects in a material. Taking into account defects, we see that phonons can only travel a certain distance before they will eventually be impeded and scatter. This concept of phonon defect scattering will lead us to be able to selectively control phonon transport in materials depending on the types of defects present, and is central to understanding thermal transport in functional oxides.

\subsection{Phonon Defect Scattering}

In determining the total mean free path, or alternatively the total scattering time associated with phonon thermal conductivity, we add all of the contributions to phonon scattering using Matthiesenn's rule, as follows 


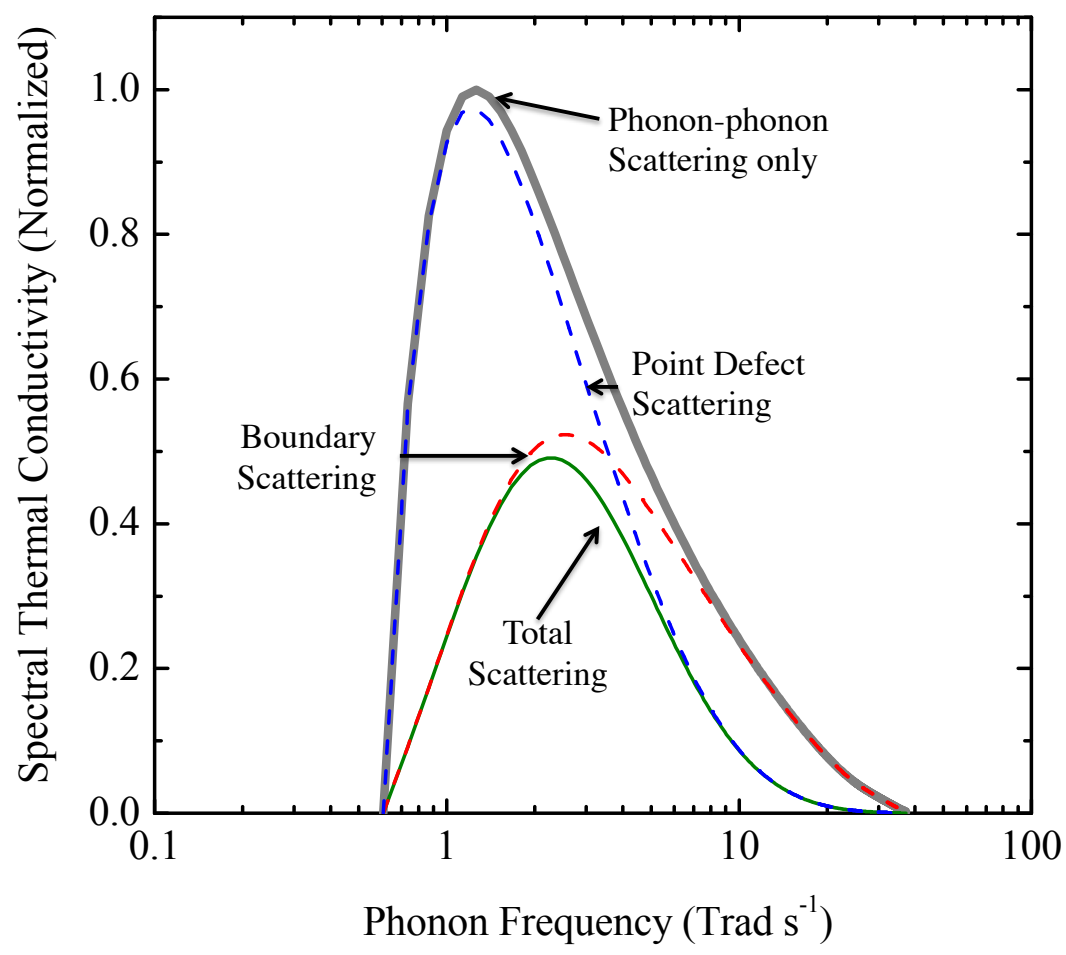

Figure 1.2: Spectral thermal conductivity dictated by the phonon wavelength (normalized to the maximum value) altered from just phonon-phonon scattering (gray, solid) by increased boundary scattering (blue, dotted), increase point defect scattering (red, dotted), and increase in both boundary and point defect scattering (green, solid). We see that different scattering mechanisms alter different aspects of the phonon spectrum, leading to a phonon filtering effect.

$$
\tau=\left(\frac{1}{\tau_{1}}+\frac{1}{\tau_{2}}+\ldots+\frac{1}{\tau_{n}}\right)^{-1}
$$

where $\tau$, the total scattering time is determined by all of the different mechanisms that can scatter phonons in a material, $\tau_{1}-\tau_{n}[22]$.

While I will discuss each scattering mechanism present in much more depth throughout this dissertation, in this introductory chapter, it is important to point out that different types of defects will scatter phonons in different ways. Point defects, for instance, will preferentially scatter phonons with high frequencies while boundaries will limit the propagation of the lower frequency, long wavelength phonons. This means that we can manipulate the intrinsic phonon spectrum such that we scatter at long wavelengths, short wavelengths, or both, and essentially create short-pass, long-pass, or notched "phonon filters" as illustrated in Fig. 1.2.

The spectral thermal conductivity is the component of the thermal conductivity that is determined by certain frequency phonons. This parameter can be determined by analyzing the phonon 
frequency-dependent components of Eq. 1 (described in greater detail in Ch. 2.2). We can visualize the phonon spectrum of a material by plotting this against the phonon frequency as in Fig. 1.2 and, given various scattering mechanisms, we see the impact of phonon defect scattering on different wavelength phonons. The area under the curve plotted in Fig. 1.2 is the total phonon thermal conductivity. Naturally introduction of additional scattering mechanisms will reduce that area. In the case of boundary scattering, we see that long wavelength, low frequency phonons contribute much less to the thermal conductivity, but the high frequency phonons are unaffected. This makes sense in that defects such as grain boundaries will stop phonon propagation if the phonons would otherwise exceed the boundary length, but high frequency phonons will already scatter on much shorter length scales such that their resistance is not dominated to that boundary. Point defects will have the opposite effect. High frequency phonons will be greatly perturbed by these defects which are on the size scale of high frequency phonon mean free paths and low frequency phonons are not impacted. As illustrated by the black dotted line in Fig. 1.2, multiple defect mechanisms can be combined to limit the spectrum of the phonons in a material and reduce the thermal conductivity in a controlled manner through phonon defect scattering.

\subsection{Thermal Transport in Functional Oxides}

As the goal of this dissertation is to determine the impact of phonon defect interactions specifically in functional oxides, it is important to introduce the characteristics of thermal transport in these materials before moving forward.

Since phonons are collective vibrations of the atoms on a crystal lattice, it makes sense that complex crystal structures will yield more complexities in phonon transport. Thermal transport in functional oxides, which by definition have at least two (and often more) atomic species involved in their crystal structure, can be significantly more complex than that in simple, monatomic materials. Two common crystal structures for functional oxides relevant to the studies that will be discussed in this dissertation and shown in Fig. 1.3 are rocksalt (e.g., $\mathrm{MgO}, \mathrm{CdO}, \mathrm{NiO}, \mathrm{CuO}$ ) and the perovskite structure (e.g., $\mathrm{BaTiO}_{3}, \mathrm{SrTiO}_{3}, \mathrm{CaTiO}_{3}$ ). While complexities in oxide atomic structure lead to significant variation in phonon behavior, we will see that it also opens the avenue for wide variability in point defect dynamics among the variety of atomic species involved.

The structures depicted in Fig. 1.3 are cubic, however non-centrosymmetric structures are also 

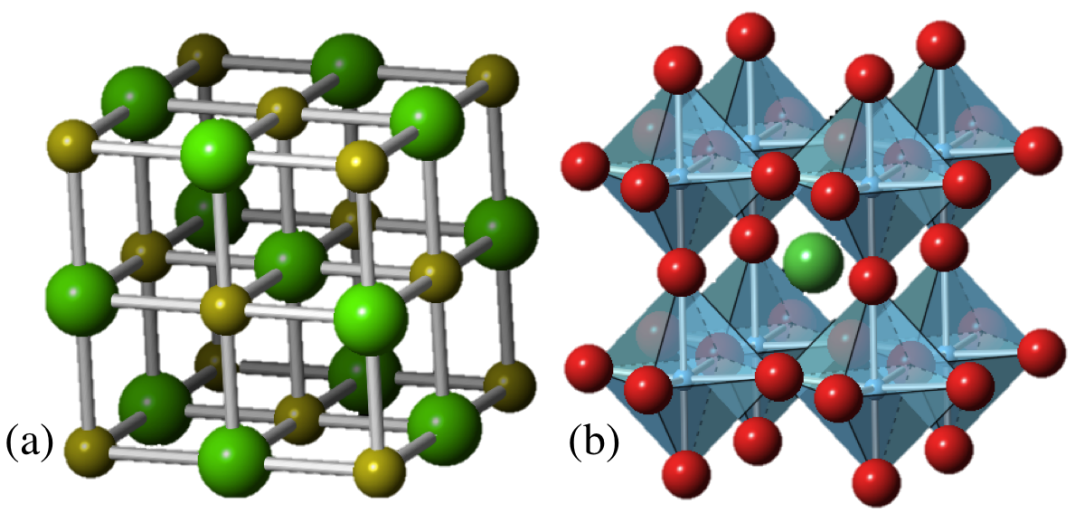

Figure 1.3: Common functional oxide crystal structures are the (a) rocksalt and (b) perovskite crystal structures. Visualized using CrystalViewer ${ }^{\mathrm{TM}}$ by CrystalMaker Software Ltd.

quite common in functional oxides. Centrosymmetry is an important property which encompasses phases that are dictated by relatively small shifts in equilibrium sites that can lead to ferroelectric polarizing behavior and give rise to electronic domains. $\mathrm{BaTiO}_{3}$ is an example of a noncentrosymmetric material. In bulk, $\mathrm{BaTiO}_{3}$ transitions from a low temperature rhomobohedral structure to orthorhombic to tetragonal just below room temperature, and finally to cubic at the Curie temperature, $393 \mathrm{~K}$, all of these phases are dictated by a small shift in the cation equilibrium locations in the unit cell [54]. The Curie temperature is the temperature below which a crystal is polarizable, whether electrically or magnetically.

Functional oxides can be fabricated via a number of different processing techniques, yielding a wide variety of quality, ranging from single crystal growth via methods such as the Verneuil process [55] or molecular beam epitaxy [56], to polycrystalline growth by methods including sintering [57] or chemical solution deposition [58]. This processing variability in itself offers the opportunity to prescribe well defined defect profiles and tune thermal conductivity. In addition, functional oxides are often processed to have specific electrical properties and can be tuned to be electrically insulating, semiconducting, or conducting $[5,29,53,59]$. With such a vast set of variables, it is not surprising that the thermal properties of these materials have been studied for decades [42, 60-69].

From a strictly thermal perspective, even stoichiometric functional oxides can exhibit a wide array of behaviors. In Fig. 1.4, I show the trend in thermal conductivity over temperature for a number of different bulk oxides. Some functional oxides behave as ordinary phonon dominated systems, with a peak thermal conductivity in the 10's of Kelvin range where phonon scattering mechanisms 


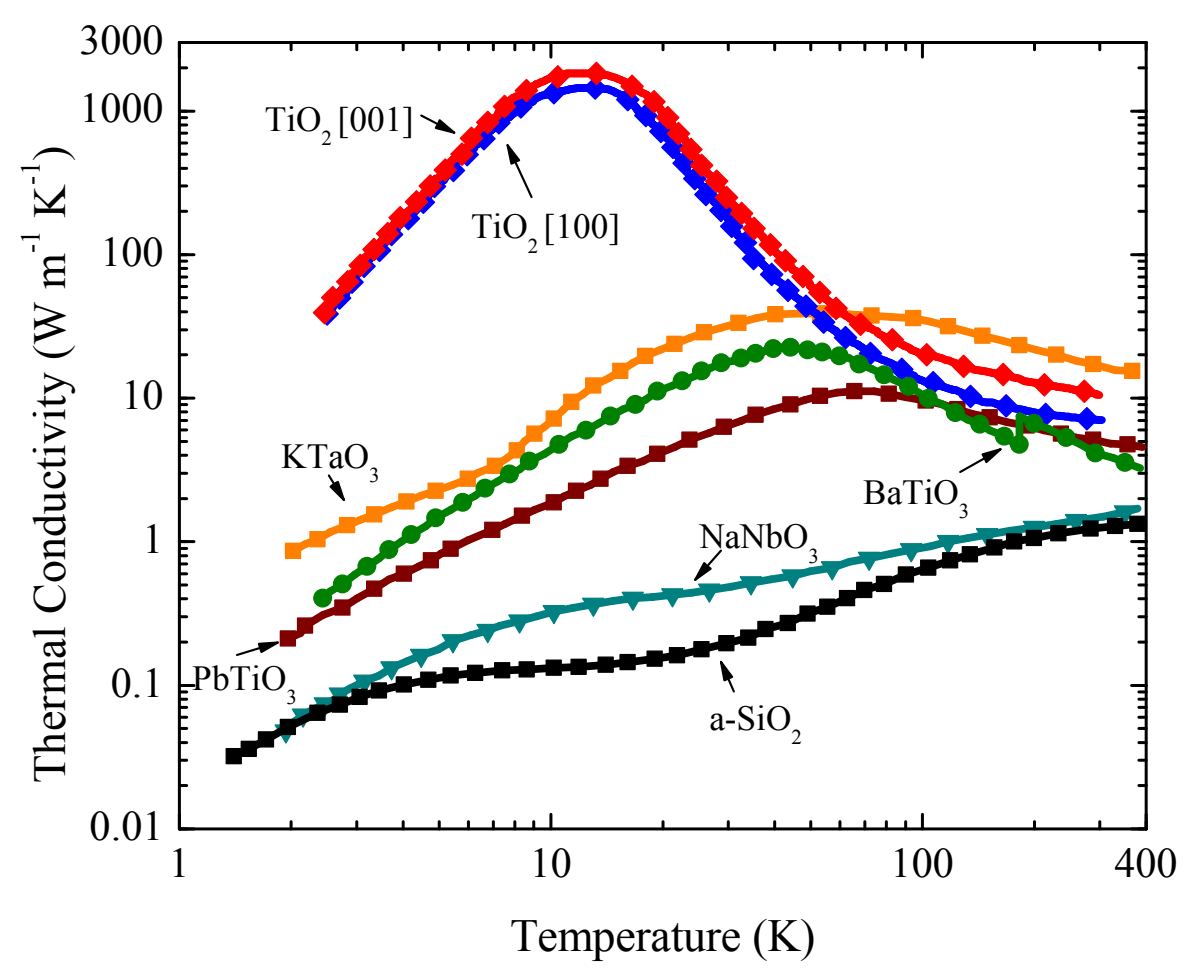

Figure 1.4: Thermal conductivity of a number of perovskite crystals (along with $a$-Si for reference) from Tachibana $e t$ al. [69] along with the rutile $\mathrm{TiO}_{2}$ along the [001] and [100] directions from Thurber et al. [15]

shift from mainly boundary and impurity limited transport to phonon-phonon scattering. There is added complexity in materials such as $\mathrm{BaTiO}_{3}$ which exhibit phase transitions, some of which have a distinct impact on thermal transport. In addition, there are functional oxides that, while not much different chemically and still fully crystalline, exhibit extremely low thermal conductivity, approaching that of typical amorphous materials.

Historically, the underlying physics of thermal transport in oxides was observed experimentally using bulk techniques involving a variety of macroscale heaters and temperature sensors [15, 61, 62, 70, 71]. This method has been somewhat standardized with tooling such as Quantum Design's Physical Property Measurement System (PPMS), which is the system used in the Tachibana et al. studies featured in Fig. 1.4 [69, 72, 73]. Another common bulk measurement technique is the laser flash method which is a transient method based on thermal decay from absorbed illumination but can be susceptible to losses from radiation, especially in high temperature measurements [74].

Theory and modeling of the phonon thermal conductivity of functional oxides have evolved alongside the advancements in experimental measurements. Much of this is covered in Ch. 2.2, but will 
be briefly discussed here in historical context. Starting in the 1950's, alongside Callaway [75-77], Klemens derived the functional form of phonon scattering with point defects, and specifically oxide vacancies $[78,79]$. He continued to refine this theory and demonstrate its application throughout his career extending his theory into thin films and polycrystalline ceramics of various compositions [65, 80-82]. With advancements in computational power, many groups went beyond the analytical models pioneered by Klemens and developed molecular dynamics simulations that accurately predict phonon behavior in complex oxides [83, 84]. These modeling techniques, along with ab-initio methods, allow for computational development of the theory revolving around phonon interaction with nano-scale boundaries $[85,86]$ and point defects $[87,88]$. It should be noted that these examples give significant insight into the physical system, but due to the complexity of functional oxides, accurate modeling of these systems is often a heavy task and leads to a relative scarcity of examples in literature.

To study the effects of defects and small scale structuring of complex materials, we turn to measurement techniques that provide micro- and nano-scale resolution. The first of these techniques to become vetted in the literature was pioneered by Cahill and based on modulated electrical resistivity and thermometry $[89,90]$. The $3 \omega$ technique, was used early on to provide thermal transport measurements in very low thermal conductivity oxides. These data were used to determine transitions in behavior between glassy and crystalline behavior, the span of which was discussed previously with regards to the data presented in Fig. 1.4. This technique provides the resolution necessary to resolve phonon dynamics in materials such as crystals with layered oxide unit cells resulting in variable bonding between layers [91] and strained thin film oxides [92]. The drawback of the $3 \omega$ technique however, is its spatial resolution, with sampling depths on the order of micrometers, it necessitates complex experimental design in order to determine properties of micro- and nano-structured materials. Furthermore, $3 \omega$ requires the precise patterning of microelectronic components on the surface of the oxide in question.

The evolution of thermal transport measurement of functional oxides in the literature has led largely to the use of time domain thermoreflectance (TDTR) to understand the more complex aspects of phonon physics in thin film and nano-structured materials. TDTR is uniquely well equipped to measure thin film systems, allowing for experimental validation of the thermal impact of novel architectures and additional complexities. In our group, we have used TDTR to measure transport in 
complex oxide systems ranging from layered single crystal systems [93] to nano-grained thin films [94, 95] and many more. As all of the materials being analyzed in this dissertation involve nanometer size scales (or in the case of the $\mathrm{TiO}_{2}$, will be used to eventually analyze thermal transport in materials with nanoscale layering), the functional oxide thermal characterization technique that I use is TDTR.

\subsection{Applications of Thermal Transport in Functional Oxides}

The fundamentals of thermal transport, defects, and functional oxides are the foundation of this dissertation. However, without applications, the motivation and focus of this work would be incomplete. The following discussion will focus on applications centered around three concepts: thermal mitigation, thermal insulation, and thermal control.

\subsubsection{Functional Oxide Applications in Thermal Mitigation}

In terms of thermal mitigation, the microelectronics industry is a major driver of technological advancement. The buildup of heat is a limiting factor to the efficient operation of large scale integrated circuits, and with Moore's law driving relevant size scales into the nanometer range, understanding thermal transport in transistor architectures is of critical importance [3].

This application was initially on the minds of oxide thermal engineers with respect to degradation and breakdown of gate dielectrics, the quintessential application of functional oxides in transistor architectures [43, 96-98]. These works highlight the consideration of the phonon response not only with degraded gate oxides, but also on hot electron collisions during operation [96, 97]. The processes of dielectric breakdown and the thermal acceleration of breakdown are a major motivator of this dissertation and as such, will be discussed in detail in Ch. 2.5.

Advances in the fabrication of gate oxide materials led to the implementation of much thinner "high-k" dielectrics, which opened up the thermal community to exploration of relatively novel materials such as halfnium (IV) oxide $[44,99,100]$. In this time period, the community experienced an increase in the attention to thermal transport in fully integrated devices [101, 102].

Analysis of thermal transport in the oxide based components of microelectronic devices has resulted in a number of technological advancements. In many device structures, a layer of $\mathrm{SiO}_{2}$ is used to electrically isolate the active layer of the transistor architecture from the substrate. Bresson et al. have proposed alternative substrate isolation schemes using oxide layers that have significantly 
higher thermal conductivities, thus decreasing thermal buildup [101]. Similarly, Maitra et al. have suggested using gate dielectric materials that are chosen specifically to take into account thermal transport [102]. Nowadays, thermal transport is often considered early in the design process, for example in the application of graphene field effect transistors $[103,104]$ or thermally assisted novel memory solutions $[105,106]$. The microelectronics industry is putting significant concentration on nano-scale thermal solutions, and with that comes similar considerations in all of the fields that feed into that application.

\subsubsection{Functional Oxide Applications in Thermal Insulation}

The other extreme of oxide thermal engineering lies not in heat efficiently exiting a material, but in engineering materials that can efficiently trap heat. The two major oxide-based applications that we see in this case are thermal barrier coatings and thermoelectric materials.

Thermal and environmental barrier coatings are the thick film layers that are used to protect turbine blades from the hot gas streams in engines [67]. The materials considerations for these coatings are often centered in thermal insulation, but must also take into account stability in harsh environments and extremely high operating temperatures [107]. Since many engines are operated in oxidizing environments, and efficiency is increased with operating temperature, oxides are the generally accepted solution for these protective coatings. Processing-wise, materials in question must be compatible with common deposition techniques, the most popular of which is plasma-spray. The most common material for thermal barrier coatings is yttria-stabilized zirconia (YSZ) which, as seen

in Fig. 1.5, has a thermal conductivity below $3 \mathrm{~W} \mathrm{~m}^{-1} \mathrm{~K}^{-1}$ at relevant operating temperatures [108]. The dependence of the thermal conductivity on the deposition technique demonstrates the role of porosity on the thermal insulation through these thick films. Air plasma spray (APS) coating results in a relatively porous material, which will exhibit additional scattering of phonons from boundaries and defects, thus yielding a very low thermal conductivity.

The intrinsic low thermal conductivity of YSZ is quite interesting in itself. YSZ is essentially heavily doped $\mathrm{ZrO}_{2} . \mathrm{ZrO}_{2}$ has many polymorphs and goes through phase changes at high temperatures, however, the introduction of yttria forces a stabilization of the high temperature cubic phase across all relevant operating temperatures. It would follow, then, that the reduced thermal conductivity of YSZ is a result largely of impurity scattering. Simulations of phonon transport in YSZ, 


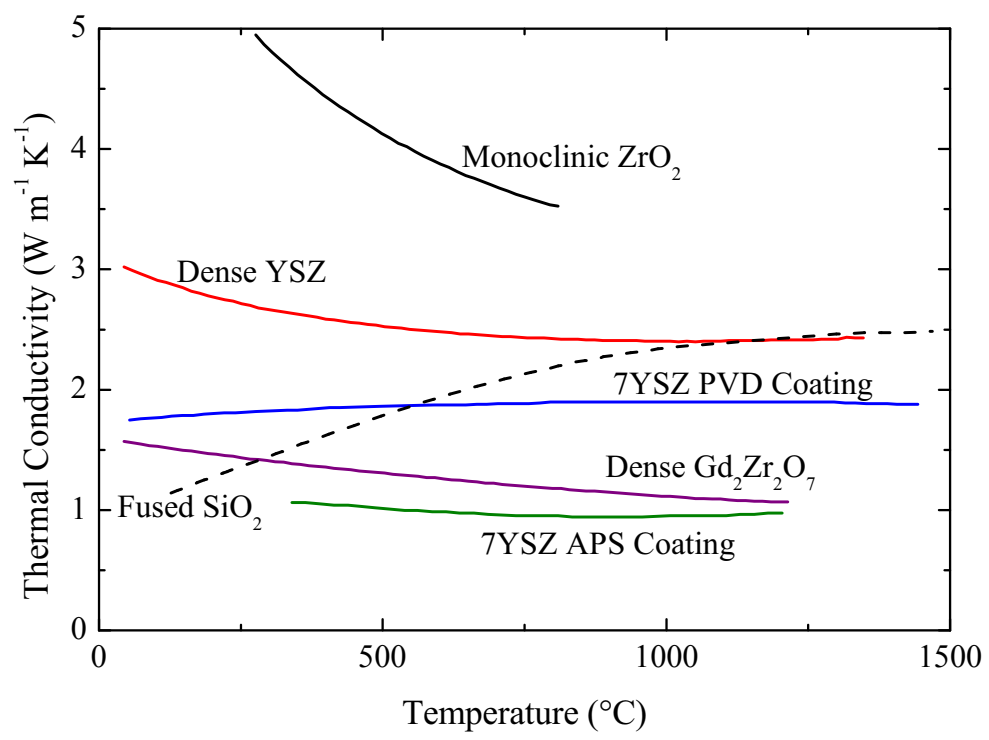

Figure 1.5: Thermal conductivity of materials used in thermal barrier coatings using various deposition techniques, all exhibiting very low thermal conductivities at high temperatures. Thermal conductivity of fused silica is included as a reference [67].

however, have actually led to the conclusion that the heat carrying vibrations in the material behave more like amorphous heat carriers than phonons [109]. This results in a very low thermal conductivity since the mean free paths of the heat carriers are extremely short and interaction between heat carriers is not dictated strictly by typical phonon interactions.

In this vain, a course of investigation for new thermal barrier coating materials lies in exploration of oxides with very complex crystal structures that will reduce phonon transport to an amorphous limit $[68,93,110]$. Additionally, investigations into adding phonon scattering mechanisms such as nano-scale boundaries have led to considering a variety of deposition techniques and materials [51, 111]. These efforts are quite challenging for a number of reasons. Operation at high temperatures puts materials well into the regime where phonon-phonon scattering dominates, so incremental changes to other scattering mechanisms may not have as profound an effect as they would at room temperature. In the commercial implementation of new thermal barrier coating materials, one must take into account many more factors aside from the phonon dynamics. Material constraints such as environmental stability and ease of manufacturing processing which directly impact the reliability and cost of the technology severely limit the flexibility in implementing new materials for thermal barrier coatings.

Alternatively, an application for low thermal conductivity materials that is recently getting a lot 
of attention and has significant potential for innovation is thermoelectric materials [17-19, 48-51, 112-127]. Thermoelectrics are materials that generate a significant electrical bias from a thermal gradient [48]. These materials are being explored for implementation in electrical generators from waste heat as well as solid state refrigerators. Since a thermal gradient is necessary to operate a thermoelectric device, having a low thermal conductivity material is crucial to the device design. For energy generation, as in any heat driven generator, the hotter the device can run the more efficient it will be, thus many materials considered for thermoelectrics are required to operate in the 100's 1000 's ${ }^{\circ} \mathrm{C}$ range. While many non-oxide materials are currently being explored for this application (common materials currently being used range from simple SiGe compounds to complex materials such as layered $\mathrm{PbTe}$ and skutterdites [18]), the combination of high temperatures and low thermal conductivities steer us towards oxides as a potential solution.

The materials component of the device efficiency is characterized by a figure of merit, $Z T$ [16]

$$
Z T=\frac{\sigma S^{2} T}{\kappa_{t}}
$$

where $\sigma$ is the electrical conductivity, $S$ is the Seebeck coefficient, $T$ is temperature, and $\kappa_{t}$ is the total thermal conductivity. The Seebeck coefficient is the proportionality constant between the generated voltage and thermal gradient (with units of $\mathrm{V} / \mathrm{K}$ ) [128]. The total thermal conductivity is determined by the combination of the contribution to the thermal conductivity both from electrical transport and phonon transport as follows

$$
\kappa_{t}=\kappa_{e l}+\kappa_{p h}
$$

where $\kappa_{p h}$ is the lattice or phonon contribution to the thermal conductivity and $\kappa_{e l}$ is the electronic contribution to the thermal conductivity, which can be related to the electrical conductivity using the Wiedemann-Franz law [129]

$$
\kappa_{e l}=\sigma L T
$$

where $L$ is the Lorenz number which is a proportionality constant $\left(L=2.44 \times 10^{-8} \mathrm{~W} \Omega \mathrm{K}^{-2}\right)$. 
From this, one can see that thermoelectrics provide an interesting materials engineering challenge. An increase in electrical conductivity results in an increase in the electrical contribution to the thermal conductivity, which will have opposing effects on ZT. Meanwhile, the mechanisms in which we use to reduce the phonon contribution to the thermal conductivity can also adversely effect the electronic mobility, decreasing $\sigma$, and potentially having significant opposing effects on $Z T$. Thus it is left up to the materials scientists and engineers to find an optimal balance of electronic mobility and phonon hinderance that will maximize the $Z T$ at a given temperature.

Complex oxides offer a tunability and control that is not seen in many other materials. Even in the mid-1900's, the thermoelectric properties of $\mathrm{TiO}_{2}$ were being investigated with modification of extrinsic and intrinsic defect levels to alter the thermopower $[15,130]$. More recently, careful processing techniques have allowed researchers to identify the role of various dopants on the thermoelectric properties of common complex oxides such as $\mathrm{SrTiO}_{3}$. Double doping effects have led to relatively high figures of merit by doping $\mathrm{SrTiO}_{3}$ with both La and $\mathrm{O}$ vacancies [119, 121] as well as La and Dy [131]. In these applications, phonon scattering from impurities is able to drive up the $Z T$ and enhance the potential for the thermoelectric efficiency of devices using these materials. Bhattacharya et al. have investigated these specific scattering mechanisms and have analyzed the role of strain field scattering of phonons in $\mathrm{Y}$ doped $\mathrm{SrTiO}_{3}$ [132]. Other dopant schemes have been investigated in $\mathrm{BaTiO}_{3}$, for instance, substitutional doping to replace $\mathrm{Ti}$ cations with $\mathrm{Nb}$ resulting in changes in thermopower [112]. Investigations into $\mathrm{TiO}_{2}$ have revealed that $Z T$ and the Seebeck coefficient can be maximized using defect engineering, moving into reduction as low as transitions into Magnéli phases $[17,133,134]$. The $Z T$ values for all of these studies mentioned have yet to exceed 0.4 by defect engineering alone, but there are other favorable aspects of complex oxides that include tuning conduction mechanisms between electrons and holes to establish n-type and p-type legs of the thermoelectric devices, relatively abundant source materials, and well known bulk processing techniques that can still maintain control over defect profiles in a scalable way.

Aside from point defect scattering, common strategies to reduce thermal conductivity for thermoelectrics lie in nano-structuring $[49,117,118]$ and introduction of nano-particles [114, 135]. Advances with respect to complex oxide thermoelectrics have been made in superlattice structures with interrupted interfaces [51] as well as incorporation of nano-scale grain boundaries and inclusions into already defected oxides [127]. 
Complex oxide materials are beginning to push into the regime where thermal and electrical engineering are enabling architectures that make for efficient thermoelectric devices. With further understanding of the interaction of phonons with engineered defects in these materials, we will be able to map out the future direction of oxides into thermoelectric and thermal barrier applications which utilize these well-known and abundant materials.

\subsubsection{Functional Oxide Applications in Thermal Tuning}

Beyond maximizing or minimizing thermal transport, functional oxide materials can also be used to tune thermal conductivity continuously. This can be extremely useful optimizing operating temperatures of nano structured electronic and energy generating devices [113, 115, 136], and is a crucial concept in the creation of thermal devices such as thermal rectifiers [137, 138].

As mentioned previously, the primary means of tuning the thermal conductivity in complex oxide materials lies in using defects to control thermal transport. While each of the studies that I present in my dissertation focus specifically on their own primary applications (multi-layer capacitors, infrared optics, and dielectric breakdown), the findings shown could easily be applied to thermal tuning applications. In functional oxide materials, we are able to introduce defects that will not only continuously decrease thermal transport, but bi-directionally tune the thermal conductivity using various defect compensation mechanisms and by introducing electronic thermal carriers.

As will be discussed in Ch. 2.3, intrinsic defect concentrations in oxides can be modified by control over the partial pressure of oxygen in growth or annealing environments. These partial pressures can be continuously controlled, thus the concentration of defects can be smoothly modulated. This was demonstrated by Luckyanova et al. where Pr-doped $\mathrm{CeO}_{2}$ was reduced continuously to map out a thermal conductivity trend that goes from intrinsic to $50 \%$ of the intrinsic thermal conductivity by annealing at various partial pressures of oxygen [139]. Bi-directional thermal conductivity tuning was demonstrated by Brooks et al. in $\mathrm{SrTiO}_{3}[140]$ by moving through a continuous range of nonstoichiometry that induces multiple layered phases (Ruddleson-Popper phases) [141, 142]. This growth was performed using molecular beam epitaxy, and so the flux of the constituent atomic species can be controlled, which enables the thermal conductivity tuning seen in Fig. 1.6 of up to an $80 \%$ reduction in the intrinsic thermal conductivity.

Utilizing molecular beam epitaxy, Sachet et al. doped CdO with Dy such that substitutional 


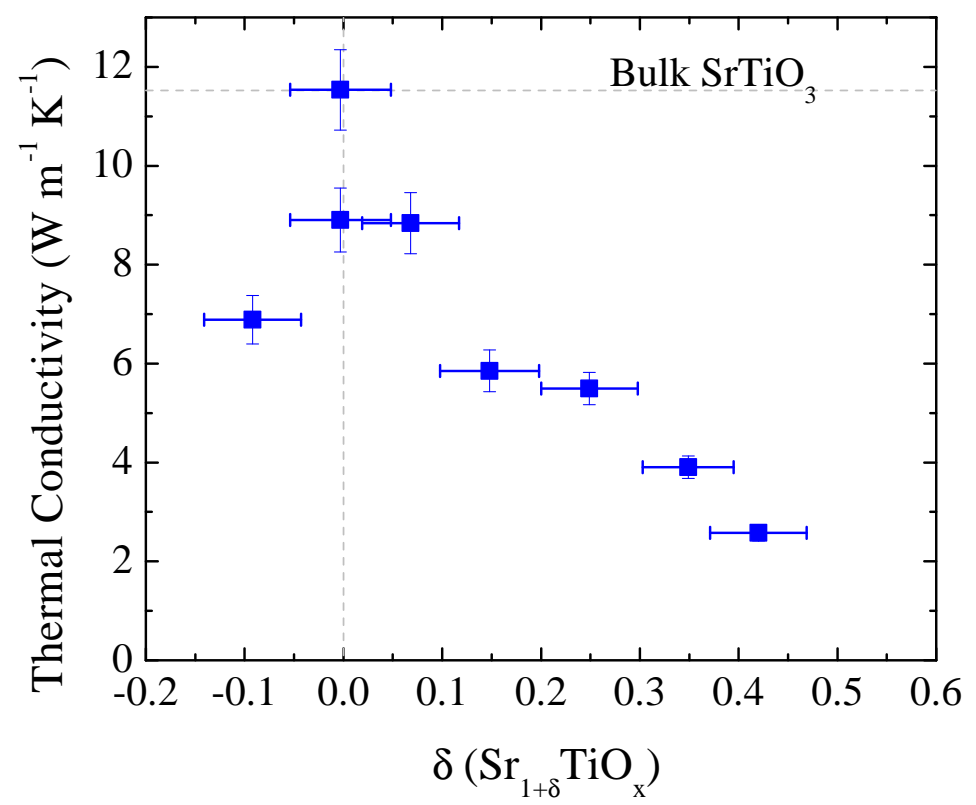

Figure 1.6: Tuning of the thermal conductivity of $\mathrm{SrTiO}_{3}$ using control over Sr stoichiometry in molecular beam epitaxy. Extreme reductions in the thermal conductivity arise from the presence of new layered phases [140].

doping increased the concentration of free electronic carriers. In this collaborative work, I demonstrated that the thermal conductivity of this system can be driven up by a factor of two beyond the intrinsic value for $\mathrm{CdO}[143,144]$. Since this work is part of this dissertation, I will explain this in much more depth in Ch. 5, however, I introduce this now since it is a prime example of thermal conductivity tuning using point defects. Specifically, it represents a unique case in which the total thermal conductivity can be tuned above the intrinsic level and then back down by using one type of dopant and the interplay of various point defect equilibria in an oxide.

Alternative tuning mechanisms lie in the continuous spanning of nano-structured dimensions that spectrally modify rates of boundary scattering. In the case of nanoscale grains, the well-known and scalable method of chemical solution deposition can be used to fine tune grain sizes in oxides spanning sizes from 10's to 100's nm grains $[58,145,146]$. This fine control over grain size enables an expansion of mobile mean free paths of phonons in the system. As seen in previous publications from our group [94] as well as the work in this dissertation [95], thermal conductivity tuning using nano-grained oxides is a viable solution to thermal transport control.

Similar effects can be accomplished using nano-scale layering found in superlattices. Oxide super- 


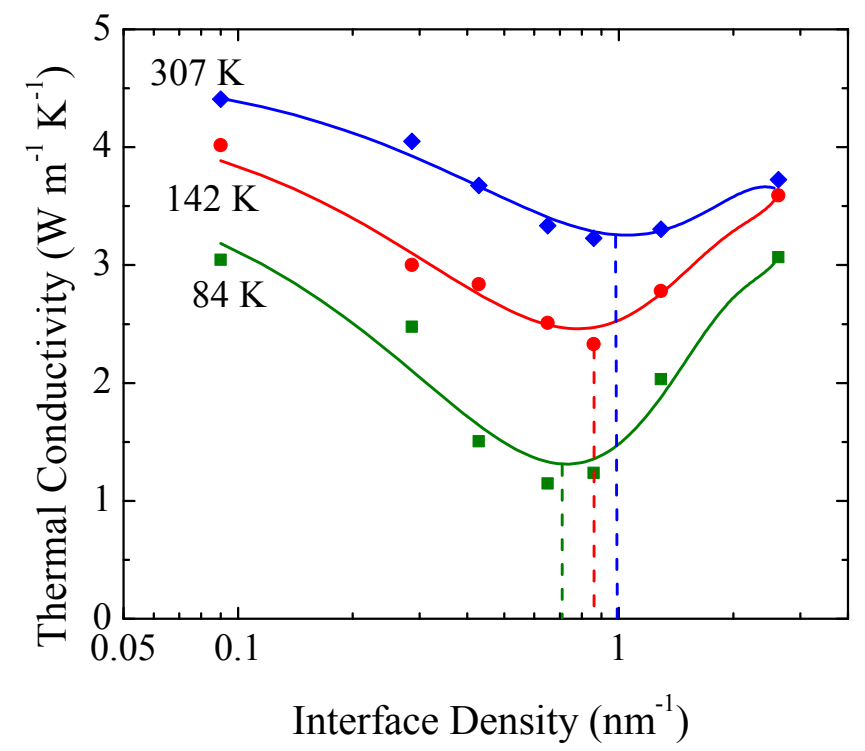

Figure 1.7: Thermal conductivity controlled by interface density of $\mathrm{CaTiO}_{3} / \mathrm{SrTiO}_{3}$ superlattice interface density at three different temperatures. Cross over into the coherent regime shifts and becomes more pronounced from changes in phonon spectrum with temperature. Lines included to guide the eye [147].

lattices exhibiting low thermal conductivites have been grown using a number of techniques including atomic and molecular layer deposition [51], molecular beam epitaxy [147], and even bulk sintering techniques for naturally occurring superlattices [91, 148]. In this case, a continuous decrease in the thermal conductivity follows the increase of boundary density. The exception to this is shown by Ravichandran et al., where bi-directional thermal conductivity tuning is achieved through a crossover into a coherent phonon regime when the interface density approaches the coherence length of the phonons [147]. Figure 1.7 shows this in the thermal conductivity of $\mathrm{CaTiO}_{3} / \mathrm{SrTiO}_{3}$ superlattices. As the interface density increases (period thickness decreases), the phonons scatter incoherently at each boundary, which results in a decrease in the thermal conductivity with decreasing boundary thickness. However, as the superlattice layers become thin enough, the system begins to act essentially as having a new unit cell that encompasses multiple superlattice layers. This resulting effect is referred to as coherent behavior with respect to phonon transport and, upon entering this regime, we begin to see the thermal conductivity increase with increasing interface density.

An extension of thermal tuning that can be accomplished with oxides is spatial thermal tuning, that is, thermal rectification. The idea behind this is to have a material, or device, with thermal transport properties that differ in a forward direction compared to a reverse direction [137]. There are many mechanisms that can be used to achieve thermal rectification, the first example of which 
was demonstrated many decades ago with an oxide-metal interface [149]. In that example, it is the electronic transfer at the interface that enables heat to move more easily from the metal to the oxide than the reverse. From a phonon standpoint, other mechanisms that are oxide-relevant have been suggested and demonstrated to accomplish thermal rectification. One such mechanism is strain gradients, where a lattice mismatch can establish a gradient in strain that will preferentially scatter phonons. This could be accomplished easily in an oxide thin film using a variety of processing techniques and graded concentrations of defects in layers.

The results of the studies shown in this dissertation can be used to determine the relevant concentrations needed to accomplish a point defect gradient induced rectification, which was demonstrated in the thick-layer limit using $\mathrm{LaCoO}_{3}$ and $\mathrm{Sr}$ doped $\mathrm{LaCoO}_{3}$ [150]. Kobayashi et al. showed that with a bi-layer heterojunction (in a bulk series configuration) the difference in the thermal conductivity between the two directions was a factor of two $\left(1.5 \mathrm{~W} \mathrm{~m}^{-1} \mathrm{~K}^{-1}\right.$ and $3.3 \mathrm{~W} \mathrm{~m}^{-1} \mathrm{~K}^{-1}$ for LCO and LSCO, respectively), and resulted in a change in thermal flux of $150 \%$ in the forward direction compared to the reverse direction. Alternative mechanisms include geometrical limitations, utilizing changes in boundary scattering to direct thermal transport and establish a gradient in the thermal conductivity. Since the relevant size scales need to be small enough to exhibit size effects, this is often seen in patterned graphene and graphene oxide that is shaped to have variable size effect based phonon scattering $[151,152]$. As will be discussed in Ch. 4, the results of this dissertation lead to a potential path forward for accomplishing geometrically graded thermal rectification using common oxide materials.

Clearly, the manipulation of thermal transport is important to a wide variety of applications. The results put forward in this dissertation will not only answer questions posed by their respective motivating applications, but can be extended to further the understanding of phonon-defect interactions in functional oxides in general.

\subsection{Statement of Objectives and Impact}

This dissertation will demonstrate the manipulation of phonon transport in functional oxides using defect scattering. Through a fundamental understanding of phonon-defect interactions and precise processing conditions, I will show that defect scattering can selectively scatter different spectral windows of the heat carrying phonons, allowing control over the thermal conductivity of complex 
oxide materials. I will dedicate the next chapter of this dissertation to discussing some fundamental ideas involved in thermal transport and functional oxides. This will be followed by a chapter on the measurement of nanoscale thermal transport. The experimental findings that I will discuss are based around three different types of oxide defects: grain boundaries, extrinsic point defects, and intrinsic point defects, each with its own chapter. Through these studies I will demonstrate the form of phonon-defect interactions, defining the thermal impact with respect to the applications of the materials as well as providing additional insight into the relevant physical dynamics by analysis of the trends in thermal transport.

- Chapter 2 - Background - In the background section I will address some key concepts in depth that will be used throughout the three studies discussed in later chapters. I will cover the definition of a phonon and the mathematical modeling of phonon thermal conductivity. I will also address the manipulation of oxide point defect profiles, a concept which is key to the final study that I will discuss. As mentioned above, I will also go into depth regarding the application of dielectric breakdown in oxides. The dielectric breakdown process is the major failure mechanism of oxides under high electric fields and directly motivates the final study of this dissertation. Each chapter covering the results of the studies conducted will also include some background on the specific subject and applications associated with that material and defect system.

- Chapter 3 - Measurement of Nanoscale Thermal Transport - The measurement technique used for the studies in this dissertation is time domain thermoreflectance. This non-contact, ultrafast, pump-probe measurement technique is ideal for the measurement of nanoscale thermal transport. In this chapter, I explain the various components that go into a thermoreflectance measurement, discussing not only time domain, but also frequency domain thermoreflectance technique as well. I will go over the physical aspects of the system, as well as a full treatment of the analysis of thermoreflectance data.

- Chapter 4 - Phonon Interactions with Grain Boundaries in $\mathrm{BaTiO}_{3}$ - Phonon boundary scattering is a crucial component to thermal engineering of nano-structured materials. In this study we analyze the effects of boundary scattering at nanometer size scales in $\mathrm{BaTiO}_{3}$, a material that is a primary constituent in multilayer ceramic capacitors and other electronic components 
that necessitate size scaling for technological advancement. We find that the grain boundaries scatter long wavelength phonons, demonstrating the spectral nature of phonons in oxides (a characteristic that has been refuted in literature focused on complex oxides).

- Chapter 5 - Phonon Interactions with Extrinsically Controlled Point Defects in CdO - Complex point defect interactions are common in oxides and can have a large impact on thermal conductivity. In this chapter, we utilize molecular beam epitaxy to control the concentrations of substitutional dopants and manipulate the defect profile resulting in tunability of the thermal conductivity of $\mathrm{CdO}$. We see that with the interaction between the extrinsic and intrinsic point defects, we are able to reduce total phonon scattering, increase total phonon scattering, increase electronic thermal conductivity, or decrease the total thermal conductivity with control simply by Dy dopant concentration. We present a model that captures the data in various doping regimes, indicating dominance of various defect interactions that occur over different dopant concentrations. This study demonstrates not only thermal tunability, but the use of thermal transport findings to further understand the complex defect interactions in oxides.

- Chapter 6 - Phonon Interactions with Intrinsic Point Defects in $\mathrm{TiO}_{2}$ - A major failure mechanism of functional oxide materials is dielectric breakdown under extreme conditions. During breakdown, large concentrations of point defects have been shown to pile up at the electrodes, which are suspected to lead to the buildup of heat from phonon scattering and accelerate the breakdown process. In this chapter, we manipulate intrinsic defect concentrations to experimentally determine the change in phonon transport properties in functional oxides. We anneal bulk single crystals under various partial pressures of oxygen to determine the impact of point defect scattering on the thermal conductivity. This study will provide insight into the changing thermal properties during the dielectric breakdown process.

These findings will help to guide the use of functional oxides in the context of thermal engineering. Whether being engineered to increase thermal gradients for energy harvesting, or to dissipate as much heat as possible under high energy density electronic operation, the trends and models presented in this work will help to provide a framework for understanding the phonon scattering mechanisms in these materials. Functional oxides provide an ideal medium to explore the manipulation of phonon transport through defect scattering thanks to the variety of processing techniques and well-known 
control of resulting defect profiles. We have shown that, using three different sample fabrication methods, in both nano-structured and bulk, and various dimensionalities of point defects, that defect control is an effective means of manipulating phonon profiles and controlling thermal conductivity.

The specific results shown in Ch. 4-6 will be published in a total of four publications. A number of other publications relating to nanoscale thermal transport and following up from these studies are listed in the final chapter of this dissertation. 


\section{$2 \quad$ Background}

This chapter will lay the groundwork and fill in the gaps of the reader as they progress on to the specific projects addressed in my dissertation. I will begin by addressing the definition of a phonon and some salient characteristics of phonons that are critical to the work that I have done. In addition, each project described in this dissertation is accompanied by insight from an analytical model. I will outline the basic process for arriving at that model in this chapter, and in each of the following chapters I will delve into the specifics that are critical for the respective findings.

Aside from thermal transport concepts, I outline two major topics regarding functional oxides. One is the formation of point defects in oxides. Point defects give rise to functionality in oxides and allow for a significant engineering opportunity. In Ch. 5 we will tune point defect profiles using extrinsic defects. In Ch. 6 we control point defect profiles using environmental controls during annealing. A somewhat advanced knowledge of point defect formation and interaction is required to understand these processes in depth, and so I will cover point defect formation in this chapter. The other oxide related topic covered in this chapter is dielectric breakdown. Dielectric breakdown is the motivating application for the work done in Ch. 6. In this chapter I delve into the prior work that has been done in the field as well as the physics behind the breakdown process. This background will help draw the connection between the study that I have performed in $\mathrm{TiO}_{2}$ and the dielectric breakdown process, namely the importance of understanding the impact of significant point defect concentrations on thermal transport in oxides.

Together, the topics covered in this chapter will provide the reader with a working knowledge of the concepts discussed throughout the rest of this dissertation. Each of the results-based chapters will also include some background information for the respective subject in a way that keeps the specifics of that subject fresh in the reader's mind throughout that chapter. In this way, the combination of common foundational knowledge in this chapter and specific motivation and supplemental background in subsequent chapters will come together to paint an impactful story for each study presented in this work. 


\subsection{Phonons}

Heat moves through electrically insulating crystals in the form of collective oscillations of atoms about their equilibrium positions [21]. These oscillations have maximum and minimum wavelengths dictated by the boundaries of the crystal and the atomic spacing between atoms, respectively. Since the vibrational characteristics are dictated by fixed atomic positions, the various modes of oscillations can be quantized into quasi-particles known as phonons.

Phonons are often conceptualized using a ball and spring model, where atoms are represented by a ball of a given mass and the bonds between atoms in a crystal are the springs. Figure 2.1 gives a visualization of this concept in one dimension. In a three dimensional crystal, there exist $3 \mathrm{~N}$ phonon modes, where $N$ is the number of atoms. These modes consist of three different polarizations; one longitudinal and two transverse. The longitudinal vibrations are essentially compressions and expansions of the bonds between atoms. The transverse modes are deviations from the equilibrium atomic position either vertically or horizontally with respect to the direction of phonon propagation. The restoring forces that keep these vibrations propagating come from the atomic bonds in the material. Bonding characteristics, along with the mass of the constituent atoms, dictate the nature of the phonon modes present.

Phonons can be classified into two types; acoustic phonons or optical phonons. In simple monatomic crystals only acoustic phonons exist, these are lower frequency vibrational modes that tend to a constant speed at long wavelengths (which is the sound speed in the crystal). If a crystal has more than one atom in its basis, then optical phonons will also exist. These are out of phase oscillations between the two different basis atoms and are generally higher frequency than acoustic phonons (on the order of magnitude of common optical frequencies) [23].

These concepts are captured well using a dispersion diagram, examples of which are seen in Fig. 2.2. These diagrams contain a wealth of information regarding thermal transport in a crystalline material, so I will use these examples to guide an explanation of some of the complexities of phonons that are important to this dissertation. Each branch in the dispersion diagram corresponds to a type and polarization of phonon mode. In the case of gold, a monatomic material with a face centered cubic lattice, there are three acoustic branches, one longitudinal and two transverse, with two different characteristic trends of wave vector vs. frequency. The dispersion diagram gives us a map of the 


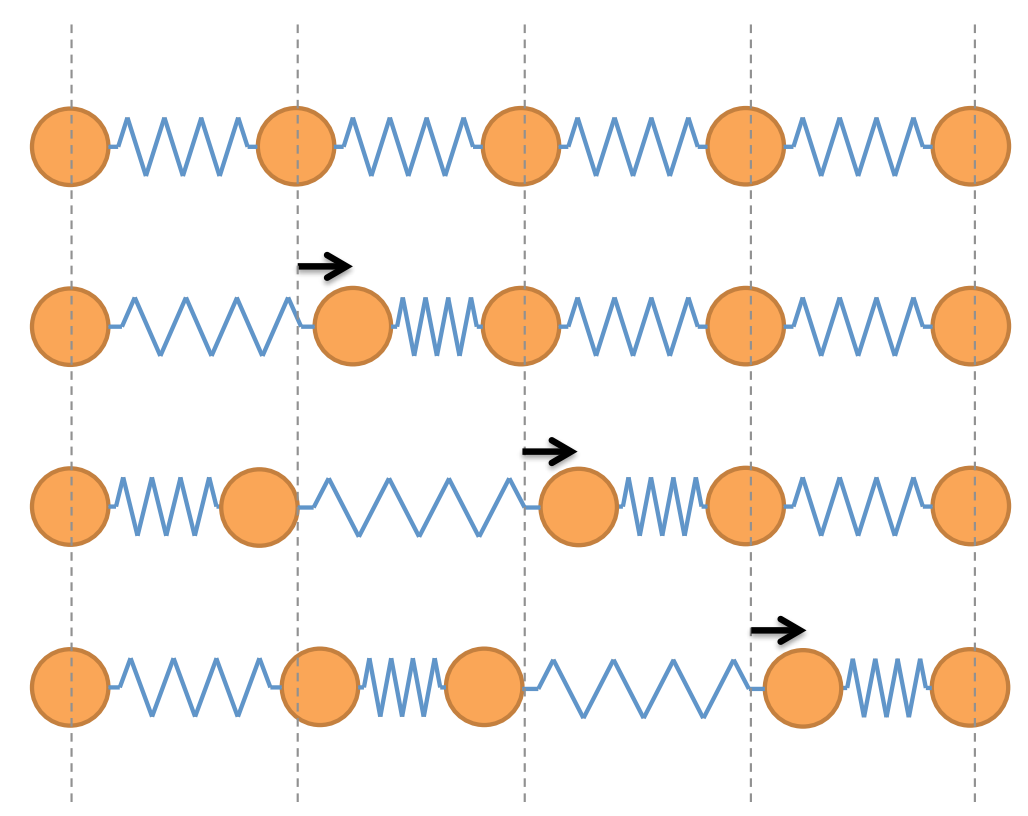

Figure 2.1: Illustration of lattice vibrations, or phonons, in a one dimensional crystal using the ball and spring model for atomic bonding.

energy of any given phonon mode with respect to the associated wave vector (or inverse wavelength). The wave vectors that exist range from nearly zero (corresponding to a long wavelength, or the crystal boundary) up to a constant defined by the lattice parameter (corresponding to the minimum wavelength). The energy of a given phonon mode, $E$, at a certain wave vector is defined in the dispersion diagram by the phonon frequency, $\nu$, and Planck's constant, $\hbar$, using the Planck-Einstein relation similar to photons where $E=\hbar \nu$.

The group velocity of phonon propagation can also be determined from the dispersion diagram as it is defined by $v=\partial \omega / \partial k$. As can be seen in Fig. 2.2, the dispersion of each acoustic branch is linear at long wavelengths, corresponding to a constant phonon group velocity, which as mentioned previously, is the sound speed in the crystal.

If there is more than a one atom basis for the unit cell of a material, then additional types of phonon modes will exist. We see this in the silicon phonon dispersion in Fig. 2.2. Aside from the acoustic phonon branches, optical phonon branches also exist at higher frequencies that correspond to $180^{\circ}$ out of phase vibrations at the zone center between the two different atoms of the basis.

Additional complexities in atomic structure of a crystal will result in more complex phonon characteristics, as can be seen in $\mathrm{TiO}_{2}$, where the phonon dispersion has 18 branches total and the two transverse phonon branches are not degenerate. I will explore this behavior in much greater depth 

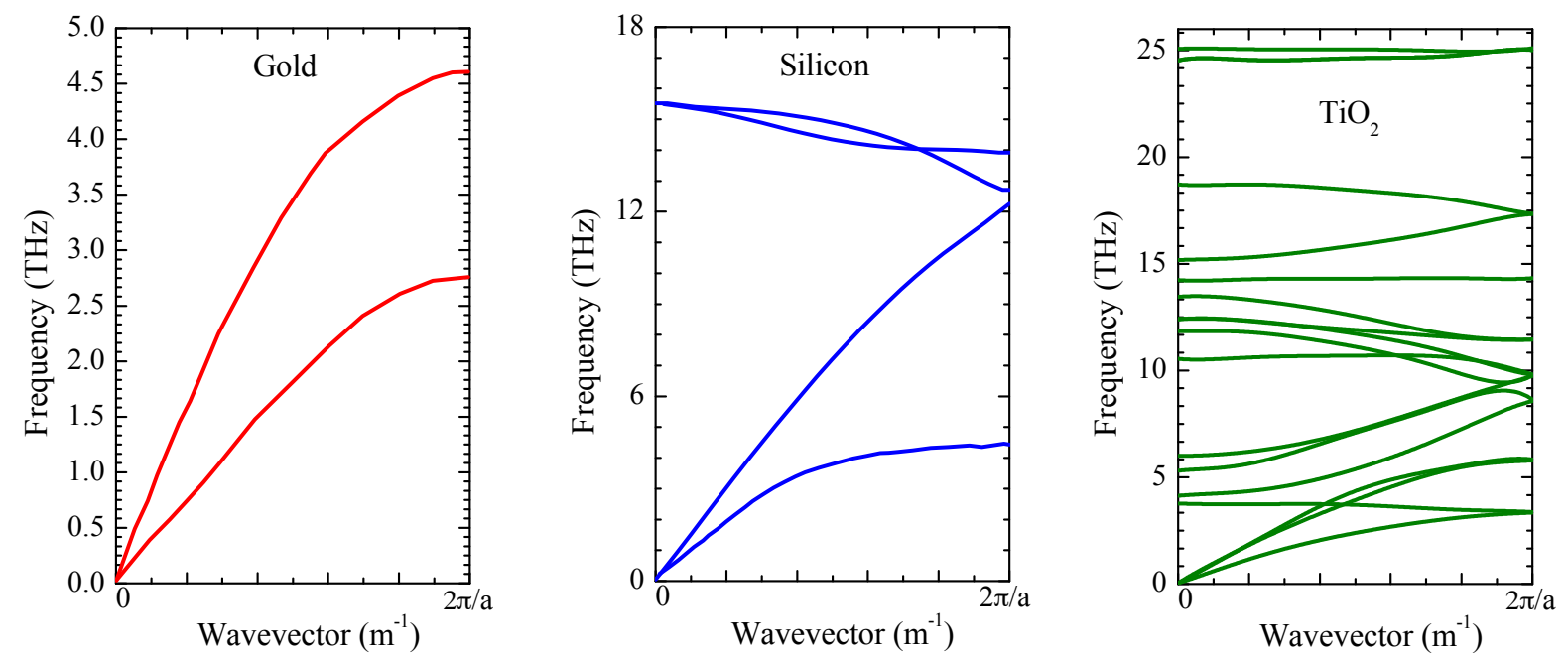

Figure 2.2: Phonon dispersion diagrams for gold, silicon, and $\mathrm{TiO}_{2}$. These materials have increasingly complex crystal structures leading to additional features in phonon characteristics [23, 153, 154].

in Ch. 6, but I will mention here that this complexity arises generally from the number of atoms in the unit cell along with an anisotropic crystal structure. The number of branches corresponds to the rutile unit cell with a six atom basis (three branches per atom in the basis) and the non-degeneracy arises from the difference in lattice parameter between the [100] axis and the [001] axis (this also results in a different dispersion relationship for these two directions).

From these basic phonon characteristics it is clear that phonon transport in functional oxides, even just considering the differences in mass and bonding of the constituent atoms in stoichiometric oxides, is quite complex and can vary significantly from oxide to oxide.

With this understanding of phonons, we can start to assemble a picture of thermal transport in functional oxides which will consist of the energy a phonon can transport, the speed at which the phonon can propagate, and the interaction that a phonon has with any non-idealities in the crystal. This dissertation is centered around the last of these three components. It is taken into account by a characteristic length that a population of phonons can travel freely without losing phase or momentum, or a mean free path. The mean free path of a phonon is dictated by a number of different physical aspects of a crystal (the presence of other phonons, the concentration of impurities in the crystal, changes in crystal structure or orientation, etc.) which will be discussed further in the following chapters. In the next section, I will show how thermal transport can be quantified in an analytical model for the phonon thermal conductivity of a crystal. 


\subsection{Modeling Phonon Transport}

In order to better understand the trends in thermal conductivity that I measure, in each study presented in this dissertation, I model the phonon thermal conductivity as a function of defect concentration. In this chapter, I will describe the general approach to modeling frequency dependent phonon thermal conductivity. I will use a simple, yet powerful and widely applied model for phonon thermal conductivity, known as the Callaway model [77]. As this is an analytical model, it lacks the complexity that approaches such as molecular dynamics or first principles models incorporate. This choice of a simple, closed form model is a conscious choice that is justified by an attempt to limit assumptions and fitting parameters that may obscure the physical nature of the model. I attempt to make few enough assumptions, limiting the model to no more than three fitting parameters, such that the model is sufficient to capture the magnitude and general trend in the data, but not over parameterized. While this may lead to fitting that appears less than ideal, it allows me to isolate important physical components of the model and identify the role they play without being masked by adjustable parameters compensating for inaccurate modeling.

Defects influence the thermal conductivity by limiting the phonon mean free path, which I model using a phonon scattering time that takes into account various defect scattering mechanisms. We combine each of these components and integrate the product over each phonon frequency and wavelength to model the total phonon thermal conductivity (i.e., the quantity that we measure). As can be seen from the chapters that follow this background, these models can capture trends in phonon transport rather well and lead to a significant improvement in understanding the mechanisms behind defect-phonon interactions in the functional oxides that I have measured.

The model I use consists of a phonon wavelength-dependent product of the energy of a given phonon mode, the velocity of this phonon mode, and the distance that this phonon mode will traverse through a crystal before scattering and losing phase or momentum [77]. The component of thermal transport that takes into account the energy of a phonon mode is the volumetric heat capacity, $C$, the phonon propagation velocity is given by the group velocity, $v$, and the distance traveled before scattering is the mean free path, $l$, which can be written as the product of the group velocity and a scattering time, $\tau$. Integrating these parameters over all phonon frequencies, $\omega$, and each branch of the phonon dispersion, $i$, yields 


$$
\kappa(\omega)=\sum_{i} \int C v l d \omega=\sum_{i} \int C v^{2} \tau d \omega
$$

This formulation will be used repeatedly throughout this dissertation.

I will start by discussing the first term in this integral, the volumetric heat capacity. The definition of the volumetric heat capacity is the change in internal energy with respect to temperature at a constant volume [52].

$$
C=\left(\frac{d U}{d T}\right)_{v}
$$

The energy in the system is the product of the vibrational energy of a given phonon mode, the density of states, and population of phonons at a given temperature. Thus the heat capacity for the phonon modes present in the material is

$$
\begin{aligned}
C & =\frac{d}{d T} \int \hbar \omega * \operatorname{DOS}(\omega) * \mathcal{F}_{B E}(T, \omega) d \omega \\
& =\int \hbar \omega * \operatorname{DOS}(\omega) * \frac{d \mathcal{F}_{B E}(T, \omega)}{d T} d \omega
\end{aligned}
$$

where $\hbar$ is Planck's constant, $\omega$ is the phonon frequency, $T$ is the temperature, DOS is the phonon density of states, and $\mathcal{F}_{B E}$ is the Bose-Einstein distribution function, which is the only component of the formulation that is temperature dependent and takes the form

$$
\mathcal{F}_{B E}=\frac{1}{\exp \left(\frac{\hbar \omega}{k_{B} T}\right)-1}
$$

where $k_{B}$ is Boltzmann's constant.

We see the form of this function in Fig. 2.3. An important aspect of this distribution is the change in frequency dependance with temperature. As temperature increases, the distribution shifts towards higher frequencies. Conversely, at low temperatures, the phonon spectrum is more heavily populated with long wavelength vibrational modes. Many phonon interactions that we will study depend heavily on phonon frequency, thus manipulation of the phonon spectrum in a material can help to identify the physical mechanisms dictating phonon transport. This concept drives the study 
of temperature dependent thermal transport in the experiments conducted in this dissertation.

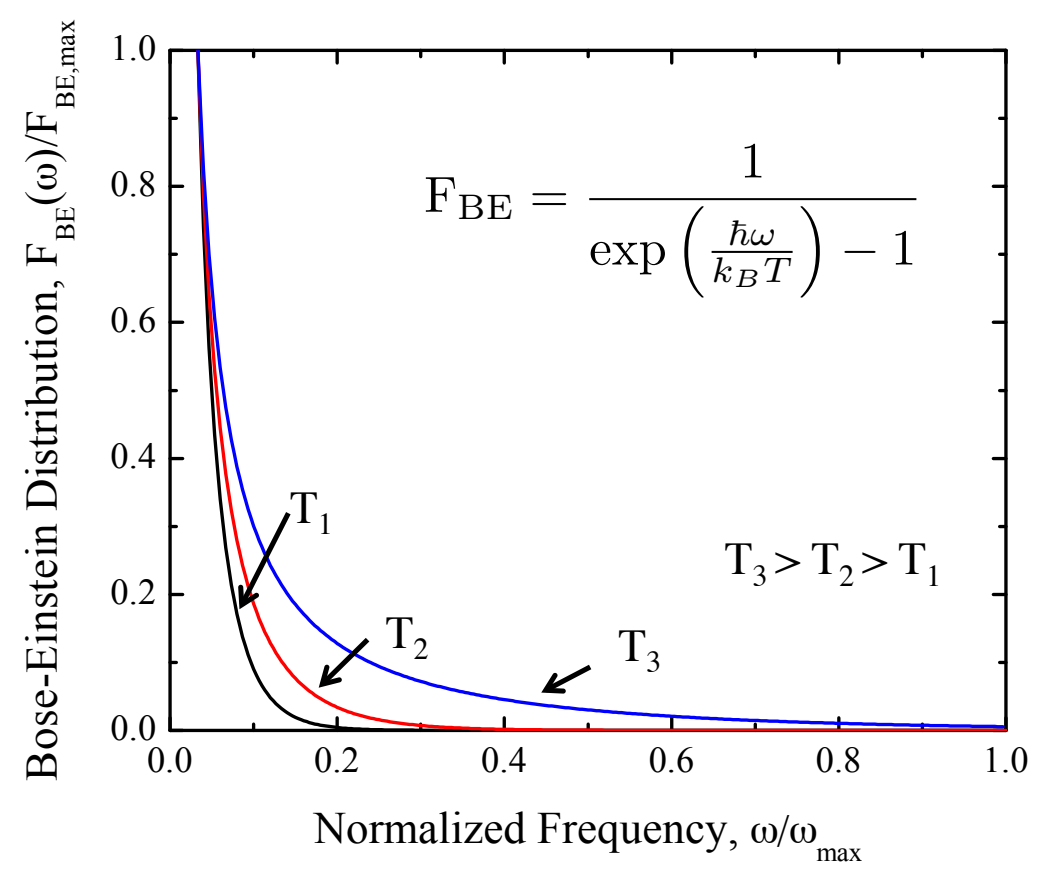

Figure 2.3: The functional form of the Bose-Einstein distribution at three different temperatures, illustrating the shift in distribution vs. frequency as temperature of the system increases.

The other major component dictating the population of phonons in a given material is the phonon density of states [21]. The spectral density of states for a three dimensional material is defined by the number of allowed states per unit volume at a given energy. Combined with the distribution of the population of phonons at a given frequency (determined from the distribution in Eq. 10), the total population of phonons at a certain frequency can be determined.

To mathematically determine the density of states we define a volume, $\Omega$, of $\mathrm{k}$-space over which we will determine the density of allowed energy states. The density of states is then the derivative of this volume with respect to energy.

$$
\operatorname{DOS}(\omega)=\frac{d \Omega}{d \epsilon}
$$

where $\Omega$ is the $k$-space volume and energy is $\epsilon=\hbar \omega$. Geometrically, this corresponds to determining the number of allowed states in a differential surface area, SA, of the volume $\Omega$ at a given energy per 
unit volume.

$$
\operatorname{DOS}=\frac{\mathrm{SA} d k}{\left(\frac{2 \pi}{a}\right)^{3} L^{3} \hbar d \omega}
$$

In isotropic crystals, we assume a spherical Brillouin zone, which yields a density of states given by

$$
\operatorname{DOS}=\frac{4 \pi k^{2} d k}{\left(\frac{2 \pi}{a}\right)^{3} L^{3} \hbar d \omega}
$$

In order to quantify the relationship between phonon frequency, $\omega$, and the wave vector, $k$, we must make some simplifying assumptions about the phonon dispersion. Various approximations of the phonon dispersion include the Einstein approximation, the Debye approximation, the sine-type dispersion, and a polynomial approximation.

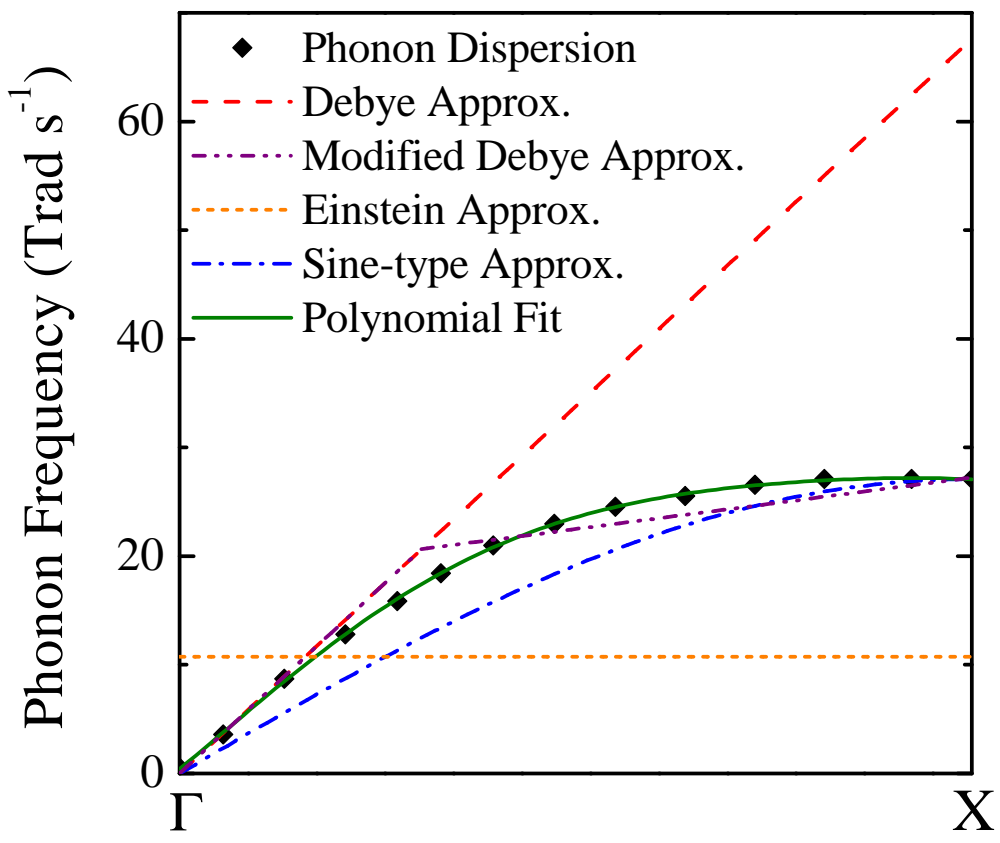

Figure 2.4: Common models used for approximating the phonon dispersion of a material, in this case the transverse branch of Si (black, diamonds), including the Einstein approximation (orange, dot), the Debye approximation (red, dash), the modified Debye approximation (purple, dot dot dash), the sine-type approximation (blue, dot dash), and a polynomial fit (green). 
The first two are named after the their creators and represent the state of understanding of vibrations in crystalline solids in the earlier part of the $20^{\text {th }}$ century. Einstein approximated a crystalline solid as a lattice of uncoupled harmonic oscillators, whereas Debye extended this approximation to take into account the coupling of atomic vibrations with their neighbors. Thus the Einstein approximation treats all of the atomic vibrations in a solid as having one frequency. With the Debye approximation, we assume that all of the phonons in the system have a constant group velocity up to a cutoff frequency at the edge of the Brillouin zone. Depending on the material system, and the phonon dynamics of interest, the Debye approximation can be a very useful first pass estimation of phonon transport. As seen in the comparison between dispersions in Fig. 2.4, the Debye approximation lacks the true dispersive nature of phonons towards the edge of the Brillouin zone. Since the thermal conductivity of a material is proportional to the group velocity (see Eq. 6), this overestimation of the contribution of these phonons to the thermal conductivity can lead to significant deviations of the modeled system from the physical system. To account for this, a modified Debye approximation is often assumed, where a prescribed transition frequency is determined at the point where the true dispersion begins to deviate from a linear relationship [155, 156]. Beyond this frequency, a second linear dispersion with a much lower group velocity is used to mimic the non-linear dispersion of the physical system.

In the work presented in this dissertation, I investigate phonon interaction with defects, which are highly dependent on phonon frequency. This leads to the necessity to go beyond these simple approximations and turn to more complex relationships that model the physical system more accurately. The first of these is the sine-type approximation, in which the acoustic phonon branches are modeled by a sine function. This dispersion relationship appropriately mimics the shift in the group velocity, with the dispersion flattening out towards the edge of the Brillouin zone. In addition, this approximation can be easily treated mathematically, as I demonstrate in Ch. 4. This approximation does not take into account the optical phonons in the crystal. In many cases, the group velocity of these phonons is small and they have a negligible contribution to the thermal conductivity.

The last approximation that I discuss is the polynomial fit to the phonon dispersion [157]. This is rather straight forward, provided the phonon dispersion for the material is available in the literature (often determined computationally with lattice dynamics and/or experimentally with neutron scattering $[154,158,159]$ ), one can fit a higher order polynomial (usually $4^{\text {th }}$ or $5^{\text {th }}$ order) to the 
data and use this polynomial in the calculations of the model. This method does not easily lead to the simple analytical solutions resulting from the Debye or sine-type approximations, but is easily handled computationally. A major advantage of using a polynomial fit to the phonon dispersion is the accurate representation of the phonon dispersion along a given, high symmetry direction of the Brillouin zone. The polynomial fit can achieve extremely close fitting to known dispersions and can be used to take into account effects of the optical phonon branches as well, which is not the case for other simpler approximations. We will employ the polynomial fit to the phonon dispersion of both $\mathrm{CdO}$ and $\mathrm{TiO}_{2}$ in Ch. 5 and Ch. 6 , respectively. Since we are interested in phonon scattering with point defects in these systems, which is known to depend heavily on the phonon frequency, it is imperative that we represent the phonon dispersion as accurately as possible.

In the remainder of this chapter I will leave the density of states component as the symbolic function $\operatorname{DOS}(\omega)$, for which the appropriate approximation mentioned above should be used in the computation of the heat capacity.

$$
C(\omega)=\int \hbar \omega * \operatorname{DOS}(\omega) * \frac{\hbar \omega}{k_{B} T^{2}} \frac{\exp \left(\frac{\hbar \omega}{k_{B} T}\right)}{\left(\exp \left(\frac{\hbar \omega}{k_{B} T}\right)-1\right)^{2}} \mathrm{~d} \omega
$$

Using this formulation of the heat capacity in terms of $\omega$, we can determine the thermal conductivity related to each phonon polarization as a function of phonon frequency.

$$
\kappa(\omega)=\int \hbar \omega * \operatorname{DOS}(\omega) * \frac{\hbar \omega}{k_{B} T^{2}} \frac{\exp \left(\frac{\hbar \omega}{k_{B} T}\right)}{\left(\exp \left(\frac{\hbar \omega}{k_{B} T}\right)-1\right)^{2}} v^{2} \tau \mathrm{d} \omega
$$

As mentioned previously, the phonon group velocity, $v$, can be determined using the phonon dispersion as follows

$$
v=\frac{\partial \omega}{\partial k}
$$

thus for each branch of the dispersion, the derivative of the frequency with respect to wave vector 
will give the group velocity for that type and polarization of phonon. A good sanity check for when assuming any phonon dispersion is to verify that the derivative of the zone center acoustic phonon branches yields literature values for the sound velocity of the given material.

The only parameter left to model the thermal conductivity is the phonon scattering time, $\tau$. The phonon scattering time is dictated by a number of different mechanisms, which include interactions of phonons with other phonons, $\tau_{P h}$, interactions of phonons with the film or crystal boundary, $\tau_{B o u n d}$, as well as the grain boundaries, $\tau_{\text {Grain }}$. Phonons also will scatter with any point defects. In any real crystal there will be a certain concentration of unintentional impurities present, which I refer to as baseline impurities, with a corresponding phonon scattering time, $\tau_{\text {Imp }}$. Depending on the application there may also be dopants or intentional added defects, referred to by the scattering time $\tau_{\text {Def }}$. Additionally, we can take into account scattering from known concentrations of vacant atomic sites by the scattering time $\tau_{V a c}$. Of course, there are many other mechanisms which influence the phonon scattering time (electron drag, domain boundary scattering, magnon interactions, etc.[160165]) so it is important to analyze the material system of interest and determine which will dominate the total scattering dynamics along with what mechanisms can be neglected without losing the physical picture of the system.

In determining the total phonon scattering time, I use Matthiessen's rule to combine the various phonon scattering mechanisms [22].

$$
\frac{1}{\tau}=\frac{1}{\tau_{P h}}+\frac{1}{\tau_{\text {Imp }}}+\frac{1}{\tau_{\text {Bound }}}+\frac{1}{\tau_{\text {Grain }}}+\frac{1}{\tau_{\text {Def }}}+\frac{1}{\tau_{\text {Vac }}}
$$

I have summarized the functional form of each of these scattering mechanisms in Table 1.

The scattering of phonons with other phonons in the system, often referred to as "Umklapp" scattering [169], is dependent on the phonon frequency as $\omega^{2}$ and has a temperature dependence that leads to a $1 / T$ characteristic in the thermal conductivity, seen in Table 1 . Phonon-phonon scattering often dominates thermal transport at higher temperatures since the phonon population increases with temperature (given by the Bose-Einstein distribution). This can be seen in Fig. 2.5 where the higher temperature thermal conductivity of bulk $\mathrm{TiO}_{2}$ decreases due to phonon-phonon scattering. To determine the impact from phonon-phonon scattering in the materials that I work with, I use fitting parameters, $A$ and $B$, and perform a least squares fit on data such as that included 
Table 1: Phonon Scattering Factors with Descriptions, Mathematical Forms, and References

\begin{tabular}{c||c|c|c} 
Scattering Type & Factor & Mathematical Form & Reference \\
\hline Phonon-Phonon & $\frac{1}{\tau_{P h}}$ & $A T \omega^{2} \exp \left(\frac{-B}{T}\right)$ & {$[75]$} \\
Baseline Impurity & $\frac{1}{\tau_{\text {Imp }}}$ & $C \omega^{4}$ & {$[76]$} \\
Thin Film Boundary & $\frac{1}{\tau_{\text {Bound }}}$ & $\frac{v}{d_{\text {film }}}$ & {$[166]$} \\
Grain Boundary & $\frac{1}{\tau_{\text {Grain }}}$ & $\frac{\nu}{d_{\text {gb }}}$ & {$[167]$} \\
Known Point Defects & $\frac{1}{\tau_{\text {Def }}}$ & $\omega^{4} x_{\text {Def }}\left[\left(\frac{\Delta M_{\text {Def }}}{M_{\text {Host }}}\right)^{2}+2\left[\left(\frac{\Delta G_{\text {Def }}}{G_{\text {Host }}}\right)-6.4 \gamma\left(\frac{\Delta \delta_{\text {Def }}}{\delta_{\text {Host }}}\right)\right]^{2}\right]$ & {$[168]$} \\
Known Vacancies & $\frac{1}{\tau_{\text {Vac }}}$ & $\omega^{4} x_{\text {Vac }}\left[\left(\frac{\Delta M_{\text {Vac }}}{M_{\text {Host }}}\right)^{2}+2\left[\left(\frac{\Delta G_{\text {Vac }}}{G_{\text {Host }}}\right)-6.4 \gamma\left(\frac{\Delta \delta_{\text {Vac }}}{\delta_{\text {Host }}}\right)\right]^{2}\right]$ & {$[80]$}
\end{tabular}

in Fig. 2.5.

At low temperatures, where phonon populations are not high enough to cause significant phononphonon scattering, the major limitations to the mean free path of phonons are boundary and defect scattering, which are temperature independent, resulting in a trend dictated by the heat capacity $\left(\propto \mathrm{T}^{3}\right)$. Scattering with point defects follows the frequency dependence of Rayleigh scattering, or $\omega^{4}$ [170]. As seen in Table 1, I use the fitting parameter, $C$, to account for scattering from an unknown baseline concentration of impurities in the crystal. In my experiments, I fit the thermal conductivity data of the control sample to determine this value for the set of samples that I am measuring.

For known impurities, on which much of this dissertation will concentrate, we formulate trends in phonon scattering that do not depend on any fitting parameters, but are dictated by the perturbations of the defects with respect to mass, bond strength, atomic radius, etc. These dependencies will be discussed in great detail in the respective results-based chapters. The scattering term maintains the Rayleigh scattering behavior and is also dependent on the concentration of defects, $x_{d e f}$, and the squares of the differences of known materials parameters such as the difference in mass between the defect and the average host atom, $\Delta M_{D e f}$, compared to the mass of the average host atom, $M_{D e f}$. Differences in atomic strain are taken into account by the shear strength, $G$, and the atomic 


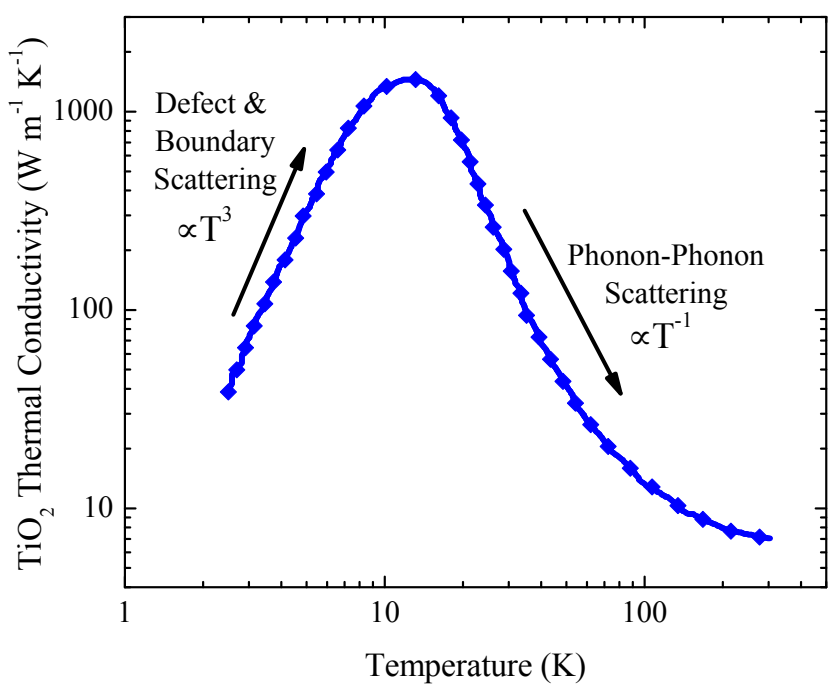

Figure 2.5: The thermal conductivity of [100] oriented rutile $\mathrm{TiO}_{2}$ over temperature with the dominant defect scattering mechanisms and temperature trends highlighted [15].

radii, $\delta$, in a similar way by the form outlined in the last two rows of Table 1 which also includes the Grüneisen parameter, $\gamma$. This scattering time is similar for known point defects and vacancies, except that the mass difference for vacancies is as follows

$$
\frac{\Delta M_{V a c}}{M_{\text {Host }}}=-\frac{M_{A}}{M_{\text {Host }}}-2
$$

where $M_{A}$ is the mass of the missing atom [81].

Boundary scattering takes a relatively simple form. Phonons scattering with film boundaries, crystal boundaries, and grain boundaries do so in proportion to the dimension of the coherent crystal. Thus the mean free path of the phonon is dictated by the coherence of the crystal, which has been demonstrated consistently in literature $[4,75,78,166,167,171,172]$, and will be shown to hold true in complex systems such as nano-grain $\mathrm{BaTiO}_{3}$.

With a formulation for the total scattering time of phonons in a system, and the use of approximations to the phonon dispersion to determine the group velocity and the heat capacity of the material, we have the full analytical picture of the thermal conductivity in a phonon dominated system. This process to determine the thermal conductivity will be used in each study outlined in this dissertation. Any variations on this formulation will be discussed in respective chapters. In Ch. 4 we will use this formulation to understand the physics of phonon scattering at grain boundaries 
of $\mathrm{BaTiO}_{3}$ by assuming a sine-type dispersion, determining baseline scattering parameters from a single crystal film, and comparing the dependance of our thermal transport model to experimental findings. In Ch. 5 we will use this model for thin film thermal conductivity of $\mathrm{CdO}$ with known substitutional Dy impurities to understand the phonon dynamics and draw additional insight into the interplay of point defect species that arise in various doping regimes. Finally, in Ch. 6 we will demonstrate that given known intrinsic defect concentrations in bulk $\mathrm{TiO}_{2}$, these models can predict thermal conductivity over a wide range of defect levels and can be used to predict thermal property changes under extreme conditions.

\subsection{Formation of Point Defects in Oxides}

A significant aspect of the presented work revolves around manipulation of point defect concentrations and their interactions with phonons. While the thermal interactions are the novel aspect of this work, it is important to understand some basic concepts with regard to point defects in oxides. In this section, we will address the thermodynamic origin of point defects in crystals and the background behind controlling point defect profiles using atmospheric controlled anneals.

The presence of point defects in materials at equilibrium is a thermodynamic phenomenon that is driven by the entropic contribution to the Gibbs free energy. In an elemental solid, the creation of a point defect, such as a vacancy, will come at a certain energetic cost, but will also increase the number of configurations that a system can take, which lowers the total energy of the system. This balance is defined by the enthalpy of formation of a defect and the configurational entropy associated with that defect formation [53].

The enthalpy of formation for a vacancy is given by the energy necessary to break the bonds surrounding an atom in a crystal and move that atom to the surface of the crystal. Since there will be an accompanied change in entropy with this process, driven by an increase in the vibrational states of the system from the introduction of the vacancy, the free energy change associated with vacancy formation, $\Delta G_{\text {form }}$, is given by

$$
\Delta G_{\text {form }}=n\left(\Delta H_{v}-T \Delta S_{v}\right)
$$

where $n$ is the number of vacancies, $\Delta H_{v}$ is the enthalpy change associated with forming a vacancy, 
$T$ is temperature, and $\Delta S_{v}$ is the change in entropy from the vacancy formation. In addition to this change in free energy of the system, formation of a vacancy will also result in an increase in configurational entropy or entropy of mixing. This configurational entropy is given by Boltzmann's equation [173].

$$
\Delta S_{c}=k_{B} \ln W
$$

where $\Delta S_{c}$ is the change in configurational entropy given by $W$, the change in the number of microstates for a given macrostate and $k_{B}$, the Boltzmann constant $\left(\sim 1.38 \times 10^{-23} \mathrm{~J} \mathrm{~K}^{-1}\right)$. In the case of a certain number of vacancies, $n$, and a certain number of lattice sites, $N$,

$$
\begin{gathered}
W=\frac{(n+N) !}{n ! N !} \\
\Delta S_{c}=k_{B} \ln \frac{(n+N) !}{n ! N !}
\end{gathered}
$$

where the factorial components of this equation can be simplified using an approximation based on large sets of numbers [174] to yield

$$
\begin{gathered}
\ln N !=N \ln (N)-N \\
\Delta S_{c}=k_{B}[(n+N) \ln (n+N)-N \ln (N)-n \ln (n)]
\end{gathered}
$$

where Eq. 24 is the previously referred to approximation known as "Stirling's Approximation". With this entropic component, we can determine the overall change in Gibbs free energy for the presence of a vacancy, $\Delta G_{v}$.

$$
\Delta G_{v}=n \Delta H_{v}-n T \Delta S_{v}-T k_{B}[(n+N) \ln (n+N)-N \ln (N)-n \ln (n)]
$$

where the equilibrium concentration of vacancies, dictated by $n$, will be given at the minimum in the change in $\Delta G_{v}$ with respect to $n$. This can be seen in the example of aluminum in Fig. 2.6 where 


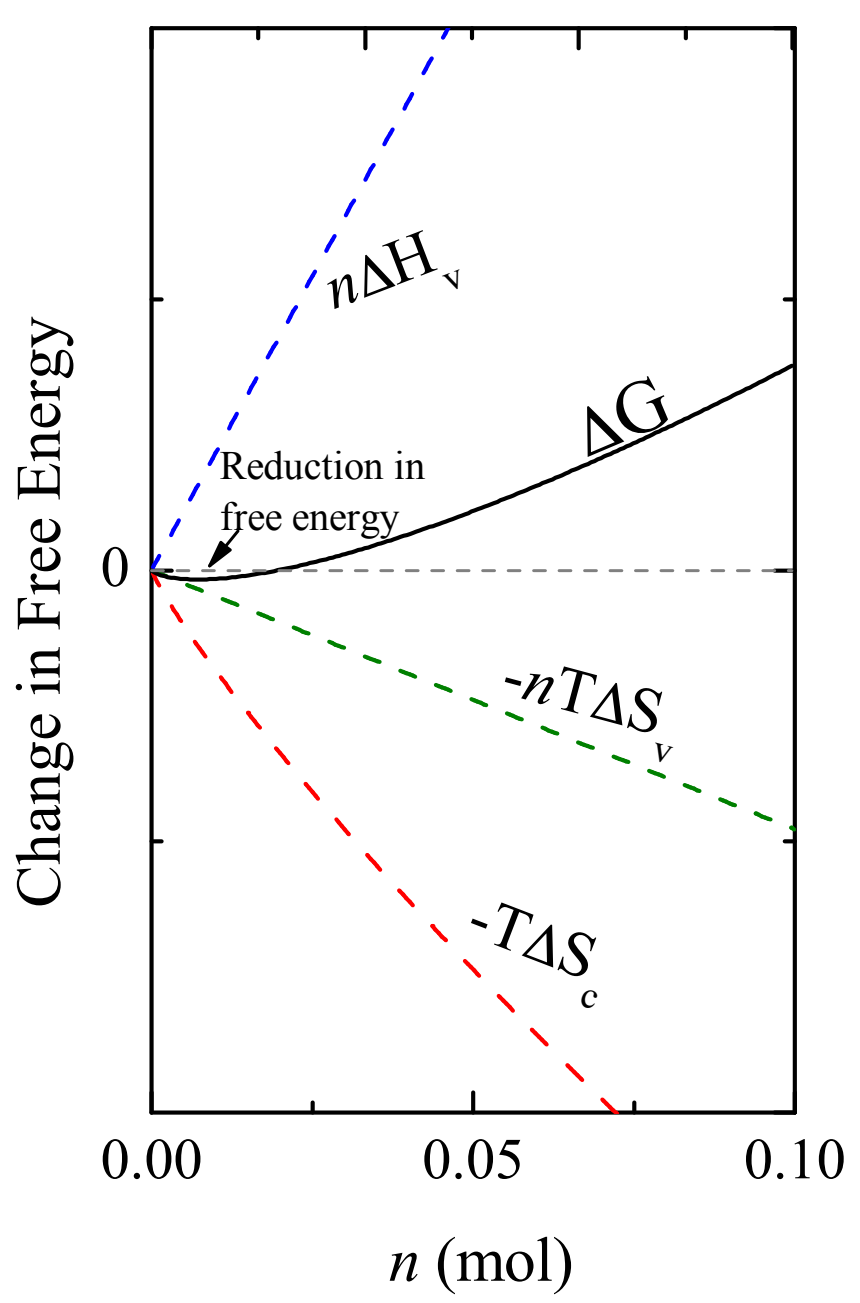

Figure 2.6: The change in the Gibbs free energy (solid, black) of Al near its melting point due to addition of vacancies along with the thermodynamic components that make up this value including the component from the enthalpy of formation (dashed, blue), the component from the increase in vibrational entropy (dashed, green), and the component from the increase in configurational entropy (dashed, red).

the separate components for the change in Gibbs free energy with respect to vacancy formation are calculated with respect to number of vacancies near the melting point [175].

This dependence of the change in Gibbs free energy with vacancy concentration, which is similar for other point defects, indicates that, as long as the system is above $T=0 \mathrm{~K}$, there will be a nonzero concentration of point defects that lowers the overall Gibbs free energy of the system. Thus, the creation of point defects such as vacancies is a thermodynamically driven process and a system in equilibrium will have a given concentration of point defects determined by the physical parameters of the material and the environment. 
In oxides, there are many types of point defects that will exist beyond vacancies. Moving forward, we will discuss these types of defects using Kröger-Vink notation where $M_{N}$ represents an atom $M$ on the crystal site where the atom $N$ should be, a vacant $N$ site is denoted as $V_{N}, M$ on an interstitial site is $M_{i}$, and the associated electronic charge is defined by superscripts of ${ }^{\times},{ }^{\prime}$, and $\bullet$ for neutral, negative, and positive charge, respectively [176]. This notation is quite useful as it allows for concise representation of the formation of various defect species in terms of defect equilibrium reactions (similar to chemical reactions) and to quickly ensure maintenance of charge conservation, mass conservation, and site conservation.

An example of one such of these defect reactions is the formation of a $\mathrm{Ti}^{4+}$ interstitial in $\mathrm{TiO}_{2}$. Considering only the $\mathrm{Ti}$ sublattice, this reaction is expressed as

$$
\mathrm{Ti}_{\mathrm{Ti}} \times \longleftrightarrow \mathrm{Ti}_{i}{ }^{4 \bullet}+V_{\mathrm{Ti}}^{4^{\prime}}
$$

where a neutral Ti atom on a Ti site becomes a quadruply positive interstitial, $\mathrm{Ti}_{i}^{4 \bullet}$, and a quadruply negative vacant $\mathrm{Ti}$ site, $V_{\mathrm{Ti}}{ }^{4^{\prime}}$.

In defect equilibrium reactions, we can use conservation of mass in the reaction to determine an equilibrium constant which is dependent on the concentrations of the constituent defects or structural components. For the reaction shown in Eq. 27, this constant would be the product of the concentrations of the resulting defects divided by the concentration of $\mathrm{Ti}$ on $\mathrm{Ti}$ sites, denoted by

$$
K_{1}=\frac{\left[\mathrm{Ti}_{i}{ }^{4 \bullet}\right]^{1}\left[V_{\mathrm{Ti}^{4}}\right]^{1}}{\left[\mathrm{Ti}_{\mathrm{Ti}}{ }^{\times}\right]^{1}}
$$

where the concentrations are denoted by brackets, and the first power is written explicitly to emphasize that this part of the mass action constant is determined by any prefactor that the component has in the defect equilibrium reaction. In this specific case, the concentration of Ti atoms on Ti sites is expected to be extremely high and can be approximated to be unity

$$
K_{1}=\left[\mathrm{Ti}_{i}^{4 \bullet}\right]\left[V_{\mathrm{Ti}^{4}}{ }^{\prime}\right]
$$

Physically, these defect equilibrium constants are associated with a change in Gibbs free energy associated with each reaction (similar to that discussed for the case of an elemental vacancy above) 
via

$$
K_{1}=\exp \left(\frac{-\Delta G_{1}}{k_{B} T}\right)
$$

where $\Delta G_{1}$ is the change in Gibbs free energy associated with the reaction given in Eq. 27.

In the case of other constituents in the reaction, we can relate the electrical conductivity to concentrations in reactions that involve the formation of charge carrying particles, such as electrons and holes. We can relate the activity of gaseous constituents in the reaction to the partial pressure of gas involved, which in this case is oxygen. This concept is demonstrated well in the case of the formation of an oxygen vacancy, given by

$$
\mathrm{O}_{\mathrm{O}} \times \longleftrightarrow \frac{1}{2} O_{2}(g)+V_{O}^{\bullet \bullet}+2 e^{\prime}
$$

where $\mathrm{O}_{\mathrm{O}}{ }^{\times}$is an oxygen on an oxygen site that is lost as half of a gaseous oxygen molecule, $\frac{1}{2} O_{2}(g)$, and has left behind a positively charged vacant oxygen site, $V_{O} \bullet \bullet$, and two electrons, $2 e^{\prime}$. Remembering that the concentration of oxygen atoms on oxygen sites is treaded as unity, we can determine the equilibrium constant for this reaction by the constituents in the product of the reaction.

$$
K_{a}=\left(p O_{2}\right)^{1 / 2}\left[V_{O}^{\bullet \bullet}\right] n^{2}
$$

where the concentration of the gaseous oxygen is determined by the partial pressure of oxygen in the environment, $p \mathrm{O}_{2}$, and the concentration of electrons is represented as the variable $n$, in accordance with literature.

Here we see that, given these equilibrium constants, we can start to formulate the trends of equilibrium defect concentrations given by partial pressure of oxygen in the environment. Experimentally, this means that by controlling the amount of oxygen in an annealing environment, and allowing the system time to equilibrate, we can dictate the concentration of point defects in a material such as $\mathrm{TiO}_{2}$.

Depending on the material in question, there will be a number of defect reactions that can be defined that characterize the defect profile of the system. For example, in the case of a generic metal-oxide, $M O$, where the metal has a valency of +2 , and the majority defect type are Schottky 
defects [53], there will be two intrinsic mechanisms that cause the separation of electrons and holes and the formation of the Schottky defects (vacant metal and oxygen sites),

$$
\begin{gathered}
n i l \longrightarrow V_{M}^{\prime \prime}+V_{O}^{\bullet \bullet} \\
n i l \longrightarrow n^{\prime}+h^{\bullet}
\end{gathered}
$$

where $V_{M}^{\prime \prime}$ is a vacant metal site and $h^{\bullet}$ is an electron hole with equilibrium constants

$$
\begin{gathered}
K_{b}=\left[V_{M}^{\prime \prime}\right]\left[V_{O}^{\bullet \bullet}\right] \\
K_{c}=n p
\end{gathered}
$$

where $p$ is the concentration of holes. Thus given equilibrium constants $K_{a-c}$ and the partial pressure of oxygen, we have four unknowns $\left(\left[V_{M}^{\prime \prime}\right],\left[V_{O}^{\bullet \bullet}\right], n, p\right)$. We can determine a fourth equation from conservation of electrical charge, given by

$$
n+2\left[V_{M}^{\prime \prime}\right]=p+2\left[V_{O}^{\bullet \bullet}\right]
$$

where the concentration of electrons and holes is dictated by the concentrations of the charge carrying defects. This final equation can be simplified in different regimes of defectivity. By moving through these regimes, and determining the relationship between the defects of interest, we can construct a full picture of the dependance on partial pressure of oxygen. These diagrams are known as Brouwer diagrams and are quite common in literature [177].

In this general example, there are three major regimes: (I) electron dominated conduction, (II) ion dominated conduction, and (III) hole dominated conduction. The corresponding electroneutrality relationships are 


$$
\begin{aligned}
& \text { I }: n=2\left[V_{O}^{\bullet \bullet}\right] \\
& \text { II }:\left[V_{M}^{\prime \prime}\right]=\left[V_{O}^{\bullet \bullet}\right] \\
& \text { III }: 2\left[V_{M}^{\prime \prime}\right]=p
\end{aligned}
$$

where we have dropped the minority concentrations from the full relationship given in Eq. 37 for each regime. Thus, in regime I, Eq. 32 becomes

$$
\begin{gathered}
K_{a}=\frac{1}{2}\left(p O_{2}\right)^{1 / 2}(n) n^{2} \\
n=\left(2 K_{a}\right)^{1 / 3} p O_{2}^{-1 / 6}
\end{gathered}
$$

where the concentration of electrons (and the oxygen vacancy concentration) follows a trend of $p \mathrm{O}_{2}^{-1 / 6}$. Similar relationships can be determined (except with a $p O_{2}^{1 / 6}$ dependence) for the concentrations of holes and metal vacancies in regime III. In regime II, we use Eq. 37 and Eq. 36 to determine the concentration of electrons (and subsequent holes) via

$$
\begin{gathered}
K_{b}=\left[V_{O}^{\bullet \bullet}\right]\left[V_{O}^{\bullet \bullet}\right] \\
n=\frac{K_{a}^{1 / 2}}{p O_{2}^{1 / 4}\left[V_{O}^{\bullet \bullet}\right]^{1 / 4}} \\
n=K_{a}^{1 / 2} K_{b}^{-1 / 4} p O_{2}^{-1 / 4}
\end{gathered}
$$

From this, and similar calculations for the other defect concentrations, we know the functional forms of the defect profiles with respect to partial pressure of oxygen and, if plotted on a log-log scale, as in Fig. 2.7, we can immediately identify the relevant slopes for the different regimes.

Given these thermodynamic equilibrium conditions, we can precisely dictate the intrinsic defect concentration by equilibrating a material, such as $\mathrm{TiO}_{2}$, at a high temperature under a certain partial pressure of oxygen. In our experiments, which will be covered in Ch. 6, we use forming gas to reduce 


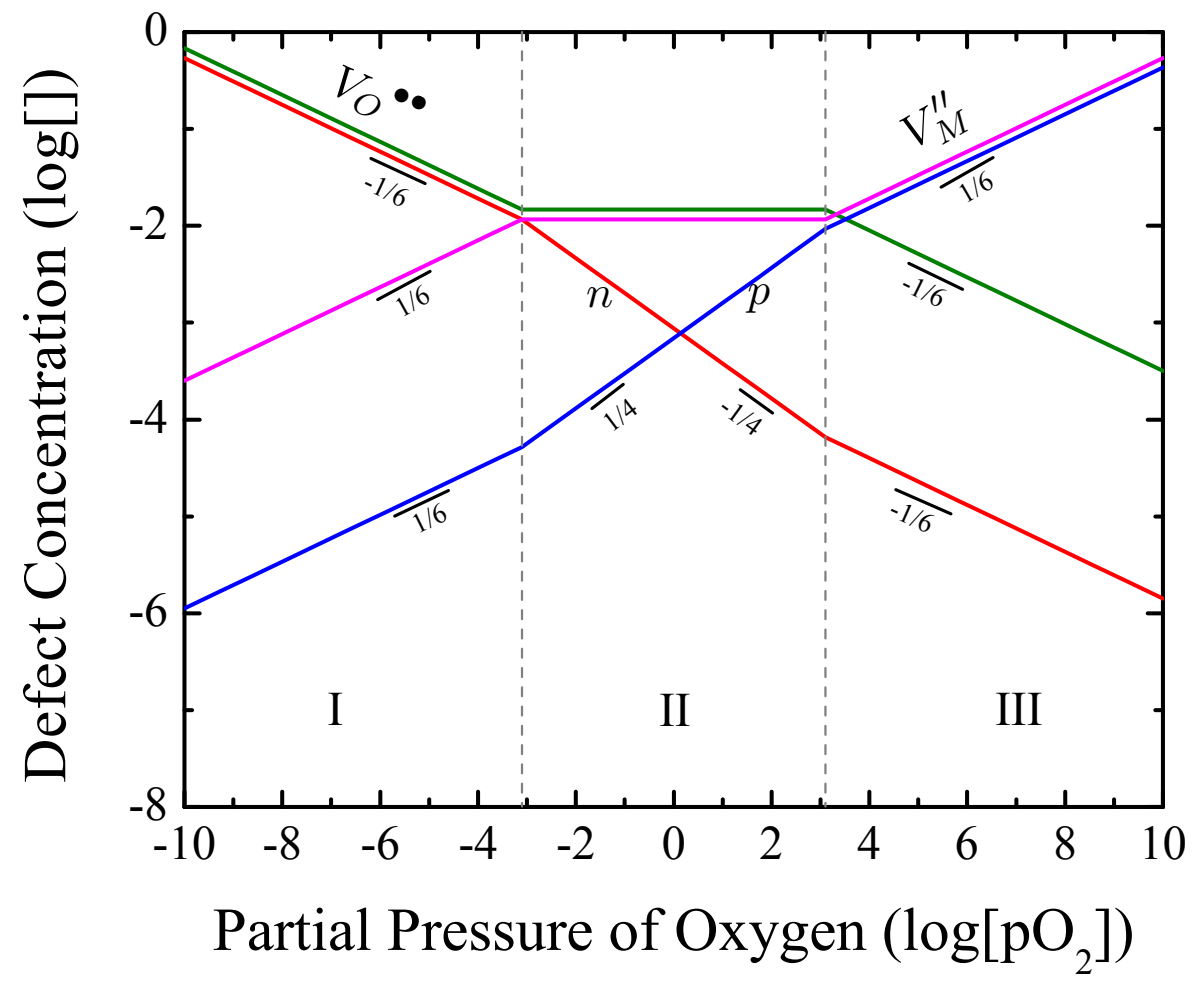

Figure 2.7: Brouwer diagram of logarithmic defect concentration vs. logarithmic partial pressure of oxygen of a general metal oxide $(\mathrm{MO})$ for relevant vacancies and the charge carrying electrons and holes. 
the partial pressure of oxygen in the environment of a tube furnace and, by annealing for long times, we can dictate the defect profile of single crystals of $\mathrm{TiO}_{2}$ to determine the impact of these defects on thermal transport. Thus we can understand the impact of interactions between phonons and the intrinsic defects in our system in a controlled way. 


\subsection{Interaction of Point Defects in Reduced $\mathrm{TiO}_{2}$}

At high concentrations, the assumption of non-interacting dilute point defects breaks down and point defects can be influenced by neighboring point defects to form larger scale order. This concept is particularly relevant to the work in this dissertation which is centered around $\mathrm{TiO}_{2}$ [178-180].

In highly reduced $\mathrm{TiO}_{2}$, the point defects aggregate along specific crystallographic planes to form what are known as crystallographic shear planes [181]. These planes are shifts in the rutile crystal structure that align along the $\{121\}$ planes. At a high enough defect concentration, the density of crystallographic shear planes reaches a point where a new material phase is formed. These new phases, with composition $\mathrm{Ti}_{n} \mathrm{O}_{2 n-1}(3 \leq n \leq 38)$, are referred to as Magnéli phases [178, 182]. Liborio et al. provide a good visualization of the crystal structure of a Magnéli phase, $\mathrm{Ti}_{4} \mathrm{O}_{7}$, by looking at a plane of cations and anions in an idealized rutile structure along the [100] direction [183]. Shown in Fig. 2.8a, the small black titanium cations and large gray oxygen anions are an idealized rutile structure. The crystallographic shear plane arrises when titanium interstitials occupy sites that cause a shift in the phase of the rutile structure, seen in Fig. 2.8b along with the line marking the (121) plane in the rutile structure.

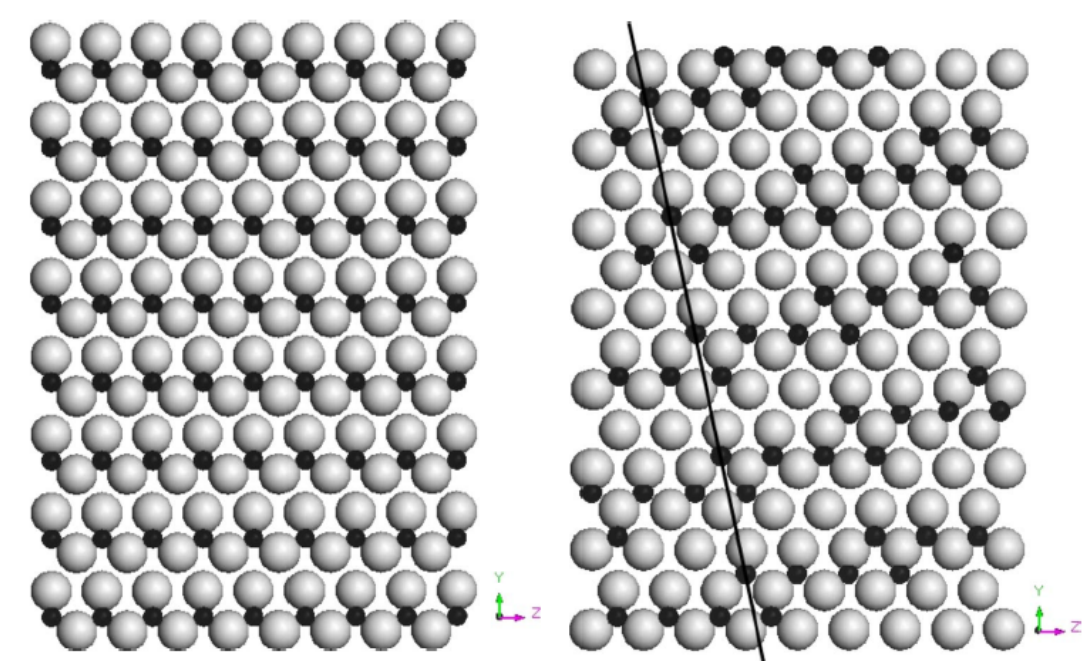

Figure 2.8: Comparison of (a) an idealized rutile crystal structure along the [100] direction and (b)the $\mathrm{Ti}_{4} \mathrm{O}_{7} \mathrm{Magnéli}$ phase with (121) oriented crystallographic shear planes defined by the ordering of titanium interstitials [183].

The extent of the sub-stoichiometry will dictate the density of the crystallographic shear planes, corresponding to many different Magnéli phases that are thermodynamically stable. These phases are the line compounds below the $66 . \overline{6} \%$ stoichiometry seen in the titanium-oxygen phase diagram 
in Fig. 2.9. Due to the variety of closely related structures, the formation of an isolated Magnéli phase from reduced $\mathrm{TiO}_{2}$ is unlikely $[184,185]$.

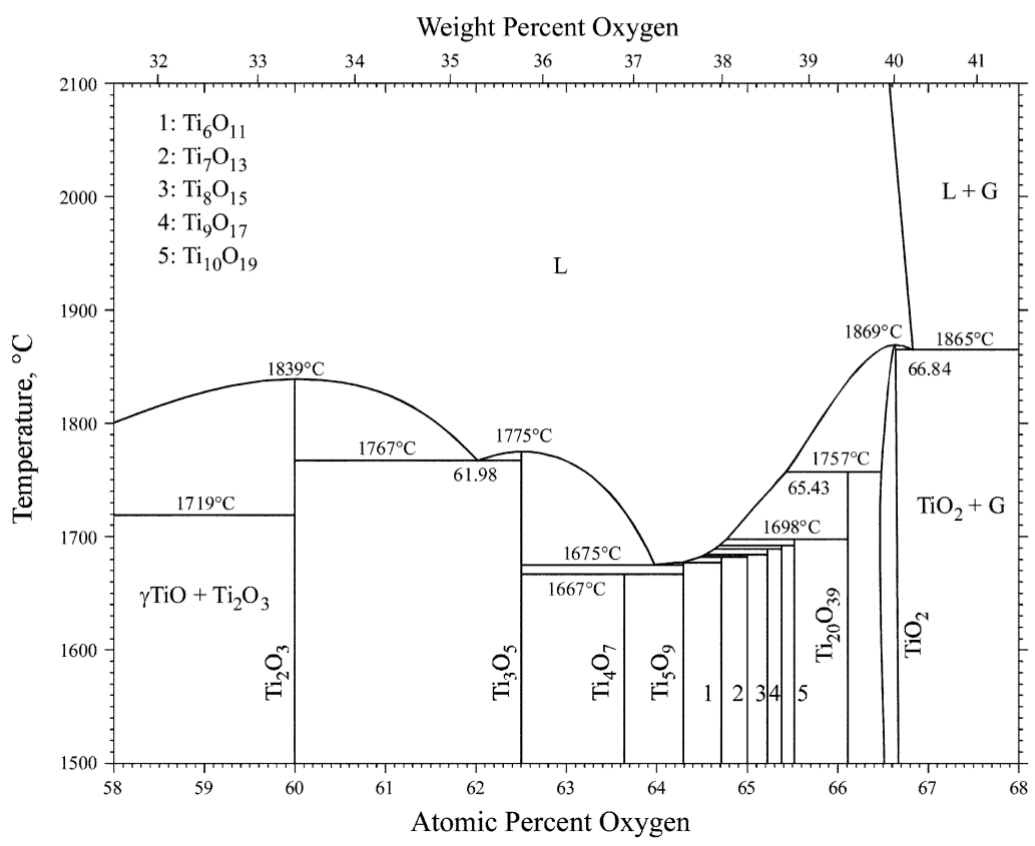

Figure 2.9: Phase diagram for the titanium-oxygen in the neighborhood of $\mathrm{TiO}_{2}$ stoichiometry. Line compounds seen at oxygen concentrations below this stoichiometry are referred to as Magnéli phases.

The exact crystallographic orientation of the layered Magnéli phase will depend on the extent of the sub-stoichiometry with the associated phase. Original studies of Magnéli phase structures analyzed layered phases that were layered parallel to the $\{132\}$ direction $[182,186]$. These layered phase orientations are a combination of the stacking faults described above which order along the $\{121\}$ planes as well as the phase boundaries that are parallel to the $\{011\}$ planes $[187,188]$. From this combination, it follows that Magnéli phase layering will occur parallel to $\{h k l\}$ planes where

$$
(h k l)=m(121)+n(011)
$$

where $m$ and $n$ are integers which will depend on the type of Magnéli phase. For example, in the case of layered planes along the (132) plane, $m$ and $n$ would both be equal to one.

The fabrication of Magnéli phases is generally done by annealing rutile $\mathrm{TiO}_{2}$ in a reducing atmosphere $[178,189,190]$. The mechanism for formation is reliant on the diffusion of titanium interstitials [191] and the lowest formation energy phase is $\mathrm{Ti}_{4} \mathrm{O}_{7}$, however achieving a sample that 


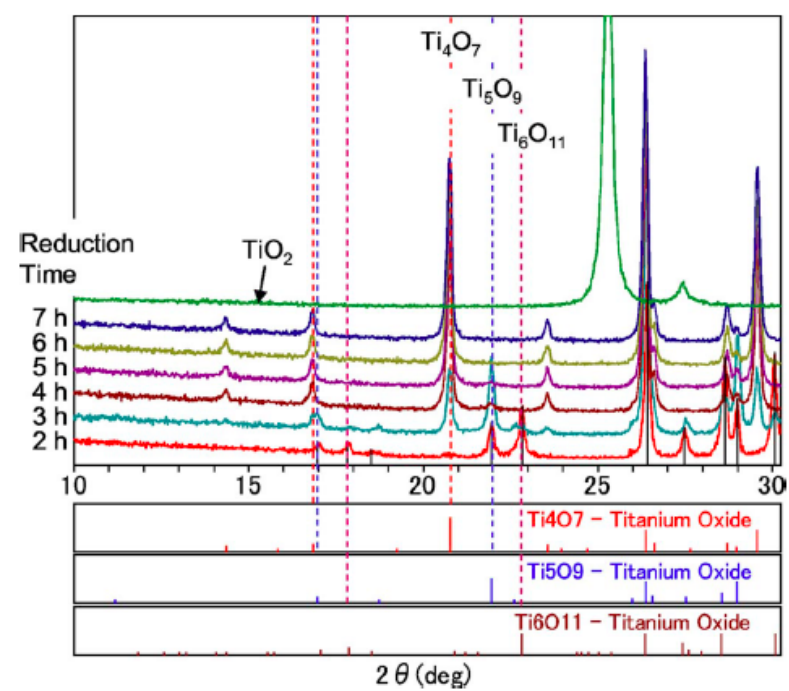

Figure 2.10: X-ray diffraction patterns of Magnéli phases arising from reduced $\mathrm{TiO}_{2}$ powders [200]

is nearly pure $\mathrm{Ti}_{4} \mathrm{O}_{7}$ has only been demonstrated by annealing for times lasting on the order of weeks $[181,188,192,193]$.

The most common characterization method for the identification of the presence of Magn'eli phases is by diffraction imaging [182, 186, 187, 190, 194-200]. In polycrystalline samples, X-ray diffraction has been used to identify the formation of of new Magnélie phases in $\mathrm{TiO}_{2}$ [190, 198-200]. Seen in Fig. 2.10, Ioroi et al. demonstrate this method of characterization while investigating $\mathrm{TiO}_{2}$ powders and are able to identify the presence of a variety of Magnéli phases that vary in concentration depending on the length of a high temperature reducing anneal [200]. In this case it appears that the nanoparticles shift to nearly pure Magnéli phase with various phases dominating at different anneal temperatures. After two hours of annealing, the $\mathrm{TiO}_{2}$ crystal structure is lost and a mix of Magnéli phases dominates the diffraction scan. At seven hours, $\mathrm{Ti}_{4} \mathrm{O}_{7}$ peaks dominate the diffraction pattern, indicating that the nanoparticles have transitioned into that specific Magnéli phase.

Transmission electron microscopy offers the ability to visualize the structure of the sample as well as analyze the diffraction pattern to gain information about crystallographic orientation. The atomic scale layering of Magnéli phases makes TEM an ideal means of investigating the structure of these materials, as seen by the wealth of such studies revolving around TEM in the literature [182, 186, 187, 194-197]. With high resolution and scanning TEM imaging, the layered nature of the Magnéli phases can be directly visualized, examples of both HR-TEM and STEM images are 


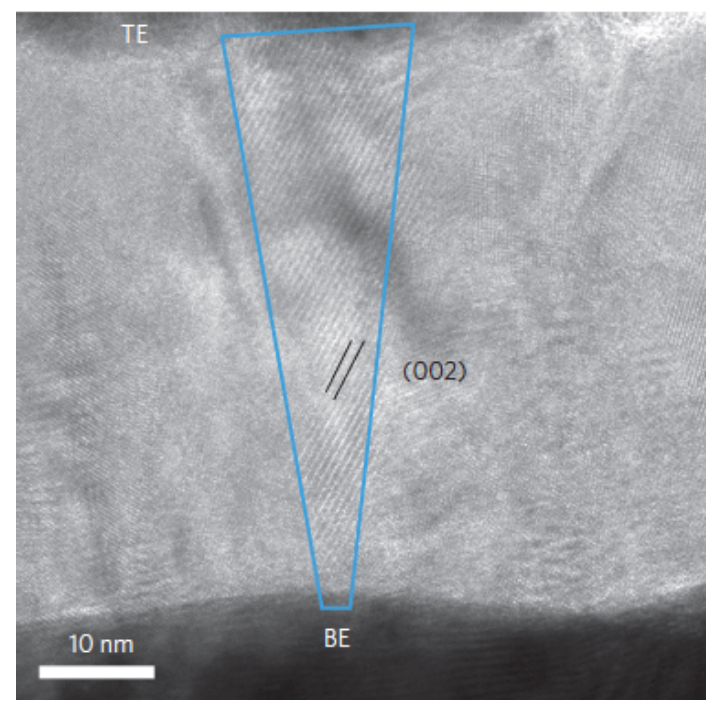

(a) HR-TEM Magnéli phase

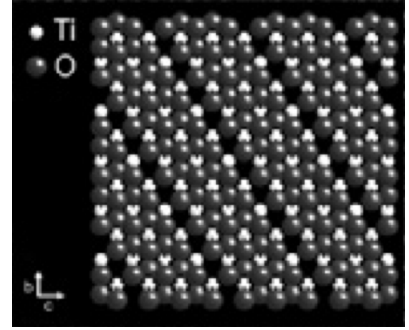

(b) STEM Magnéli phase

Figure 2.11: TEM visualization of the structure of a Magnéli phase seen (a) by high resolution TEM [195] and (b) in a scanning TEM [197]

included in Fig. ??. The HR-TEM image shows the layered Magnéli phase which has formed in a thin $\mathrm{TiO}_{2}$ film as a conducting pathway between two electrodes [195]. The STEM image gives a clear atomic view of the periodic layering of the Magnélie phase structure [197]. In this image, the periodic planes of oxygen vacancies along the [011] direction can be clearly seen.

In addition to direct visualization, TEM offers selected area electron diffraction (SAED) to verify the existence of Magnéli phases as well. Evidence of these phases is seen in the form of crystallographically oriented features (streaking and weak diffraction peaks) in addition to the primary peaks in an SAED image $[182,186,194-196]$. An early example of this is seen in Fig. 2.12, where the presence of Magnéli phases parallel to the $\{132\}$ planes manifests itself as streaks made up of weak intensity peaks oriented in the corresponding directions in the diffraction pattern [182].

Common applications for Magnéli phase $\mathrm{Ti}_{n} \mathrm{O}_{2-n}$ are in the form of ceramic electrodes [185, 200204]. The high concentration of oxygen vacancies results in significant electrical conductivity, which coupled with the corrosion resistance of the oxide make Magnéli phase oxides desired materials. Additionally, with the ability to decrease phonon thermal conductivity by defect scattering while increasing electrical conductivity, Magnéli phase $\mathrm{Ti}_{n} \mathrm{O}_{2-n}$ can be used in thermoelectric materials engineering as well $[133,134,205,206]$.

In Ch. 6, these layered phases will play a large role in the limitation of thermal transport in 


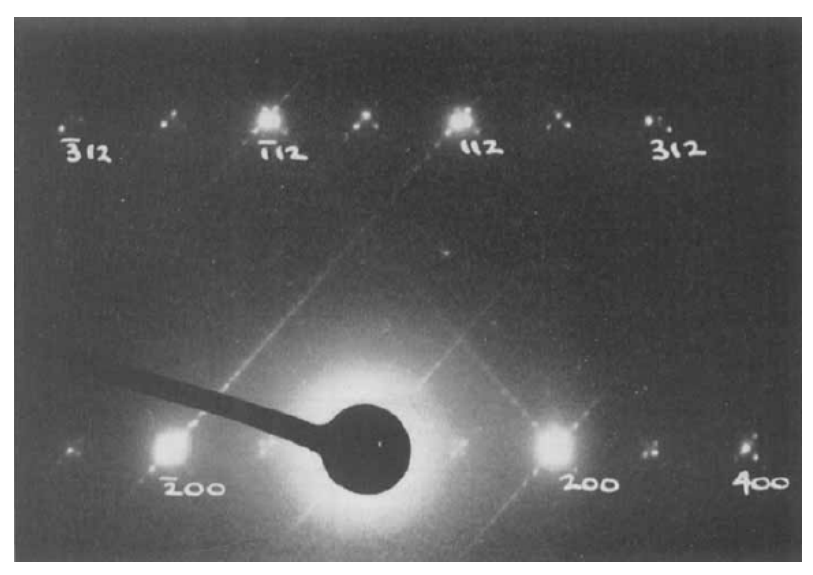

Figure 2.12: Electron diffraction image of Magnéli phase in rutile with clear signature of streaking between peaks and secondary weak diffraction peaks associated with a layered phase parallel to the $\{132\}$ crystallographic planes [182].

reduced $\mathrm{TiO}_{2}$. Previous studies that have showed changes in thermal conductivity due to substoichiometry and Magnéli phases in reduced $\mathrm{TiO}_{2}$. Early studies showed a reduction of the thermal conductivity with annealing at high temperatures for long times [15, 64]. More recent studies have endeavored to better understand the physical dynamics behind these observations [81]. Motivated by the ability to control thermal transport by phonon scattering in the layered Magnéli phases, Portehault et al. induced sub-stoichiometry via spark plasma sintering and achieved thermal conductivities ranging from $1 \sim 1.6 \mathrm{~W} \mathrm{~m}^{-1} \mathrm{~K}^{-1}$ [207]. Harada et al. utilize hot pressing of $\mathrm{TiO}_{2}$ and $\mathrm{TiO}$ powders in order to induced the formation of Magnéli phases and measure thermal conductivity as low as $3.15 \mathrm{~W} \mathrm{~m}^{-1} \mathrm{~K}^{-1}$.

The study presented in Ch. 6 of this dissertation will utilize comparative thermal modeling in order to highlight and help isolate the effects of Magnéli phase formation and defect agglomeration on the thermal conductivity of reduced rutile. Furthermore, by controlling the defect concentration using the partial pressure of oxygen in the annealing environment, we can use single crystal samples to further exaggerate the effect of the formation of off-axis phase formation on thermal transport to help identify the role of defect agglomeration in phonon scattering. 


\subsection{Dielectric Breakdown}

A major failure mechanism of dielectric materials in microelectronics, and many other common applications, is dielectric breakdown. This process, in which conducting pathways are established through normally insulative materials, can result in catastrophic failure. As this failure mechanism is the major motivation behind the final study in my dissertation, I will go into some background behind the dielectric breakdown process. The findings of my work, assessing the impact of defects on thermal transport, will aid in determining the thermal profile of materials that are undergoing extreme fields and defect redistribution prior to full dielectric breakdown.

The first major advances in understanding dielectric breakdown in solids came in the 1930's with the study of primitive bulk dielectric materials such as ordinary paper [208, 209]. These studies brought about the general observations of dependance on electric field and temperature with respect to the minimum voltage necessary to induce breakdown. Zener [208] outlines the concept of cascading excitation of electrons and Whitehead and Nethercot [209] are the first to pay specific attention to the thermal instabilities at play during the breakdown process. Whitehead and Nethercot note that above a critical temperature there is a change in the dielectric breakdown characteristics.

These concepts were revisited with respect specifically to electronic materials in the 1970's and 80's as the microelectronics boom was beginning to take off [43, 210-213]. During this time, advances in characterization technology had allowed for studies that identified the various regimes of dielectric breakdown. Budenstein puts forth a progression of the process that proceeds as formation, "tree" initiation, "tree" growth, and then a return streamer [212]. These concepts were developed using then-novel imaging techniques of scattered light collection during the breakdown process, leading to images such as Fig. 2.13 which shows the fractal-like propagation of the aptly named "tree" initiation stage of dielectric breakdown.

This demonstrates the advancements in understanding of processes such as dielectric breakdown that are available with new characterization techniques. The layout of a multi-step breakdown process makes the issue more tangible, allows for subsequent research to address specific issues, and advances the understanding of the field in general [213]. In the work shown in this dissertation, we will address the change in material properties during the formation stages of dielectric breakdown; the stages that are the precursors to the onset of the catastrophic failure process. 


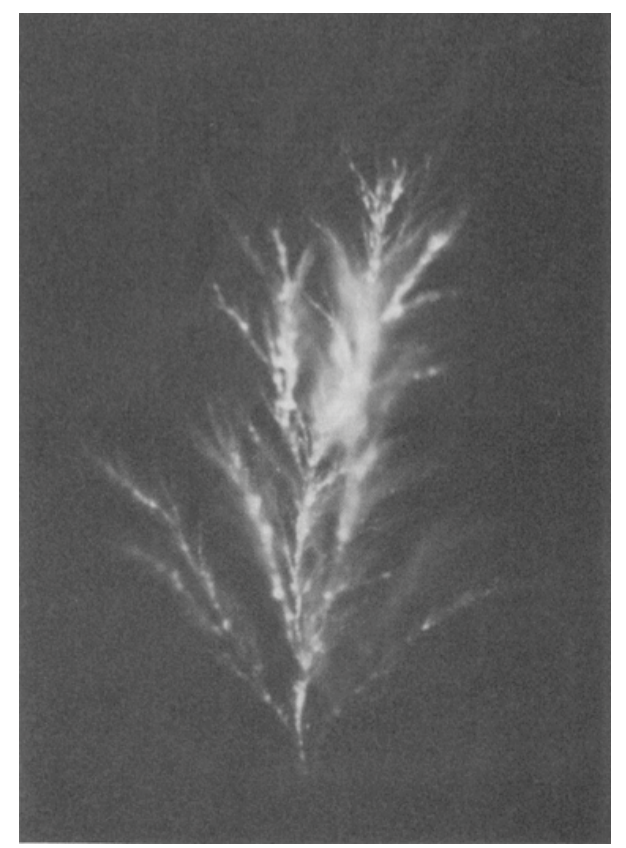

Figure 2.13: Photograph of the "tree" initiation stage of dielectric breakdown using backscattered light into a microscope $[212]$.

The issue of dielectric breakdown in manufacturing applications brought about empirical studies that utilize relatively large amounts of data to identify root causes and understand physical mechanisms of breakdown [43, 211]. These experiments yielded a number of interesting results showing the dependence of time, thickness, device area, device structure, and operation history on breakdown characteristics. The relationship between breakdown and operation history, demonstrated in Fig. 2.14, serves as further motivation that defect migration and long term material rearrangement plays a significant role in breakdown. In this example, Anolick and Nelson show that breakdown threshold decreases substantially after long term, sub-breakdown operation [211]. While many different mechanisms are likely at play in this result, the drift of point defects and resulting changes in material properties are expected to play a large role in this change.

This research began a trend towards understanding the physical and materials mechanisms which lead to dielectric breakdown, and it is DiStefano [214] who emphasized the role of ionic diffusion in the formation and development of dielectric breakdown. This concept has been shown in more recent work to be a fundamental part of the breakdown process and work presented in this dissertation is motivated by exploring the effects of this process $[197,215,216]$. O'Dwyer highlighted the thermal aspects of dielectric breakdown [210,213]. His findings show that high temperatures may not directly 


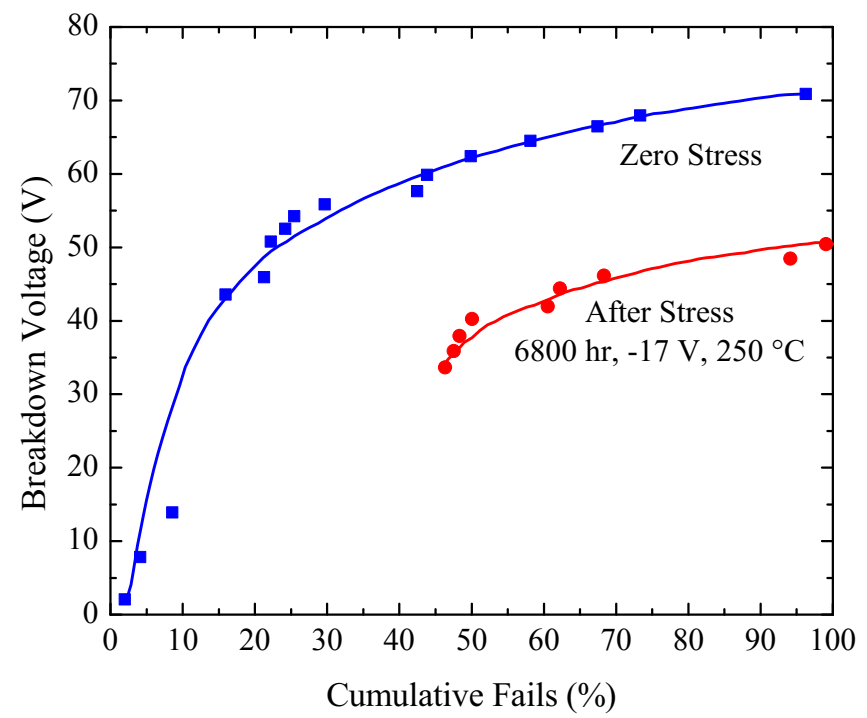

Figure 2.14: Probability of failure and breakdown voltage for samples before operation (blue, square) and after a significant time of operation at below-breakdown conditions (red, circle) [211]. Curves included to guide the eye.

cause dielectric breakdown, but lead to an acceleration of the process and can lower the dielectric breakdown threshold.

As the microelectronics community realized the need to replace $\mathrm{SiO}_{2}$ gate dielectrics in transistors, research into alternative materials, and thus the influence of various material properties on dielectric breakdown, led to substantial new understanding [215, 217-220]. One of the early materials tested was $\mathrm{TiO}_{2}$, where Kim et al. demonstrated a nice example of breakdown threshold testing, looking for the sharp discontinuity in a current vs. voltage curve, seen in Fig. 2.15 [219].

Digging deeper into the physical mechanics behind dielectric breakdown, McPherson et al. investigated a wide array of dielectric materials and determined that there is an inverse square-root relationship between breakdown strength and dielectric constant. Others have shown that the breakdown strength of a material can be improved significantly using nano-structuring [221-223]. Through this evolution in the understanding of dielectric breakdown, we can extract an agreed upon general form of the time to failure due to dielectric breakdown,

$$
t_{\text {fail }}=\exp \left(\frac{\Delta H_{0}}{k_{B} T}-\gamma E\right)
$$

where $t_{\text {fail }}$ is the time to dielectric breakdown, $\Delta H_{0}$ is the activation energy for bond breakage in the solid, $k_{B} T$ is the thermal energy in the system given by the product of Boltzmann's constant and 


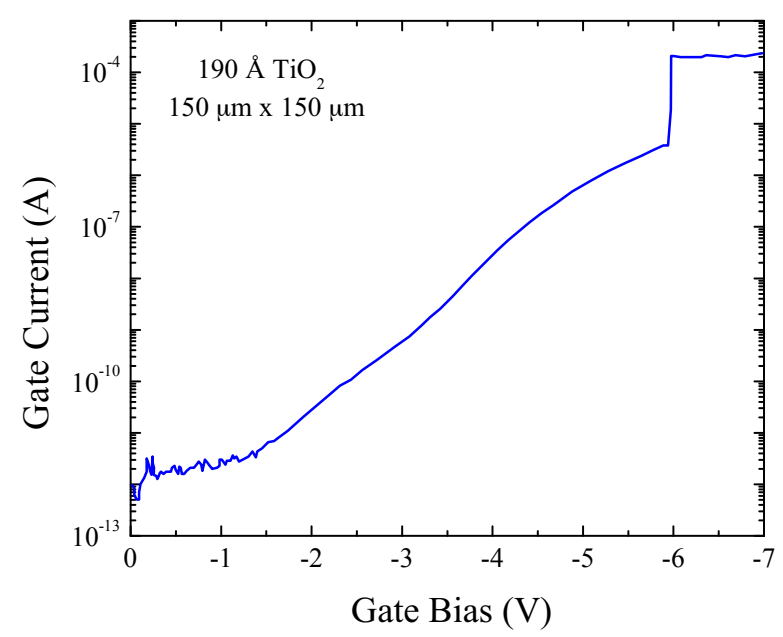

Figure 2.15: Example of a measurement of dielectric breakdown, conducted by Kim et al. on $\mathrm{TiO}_{2}$ gate structures $[219]$

(a)

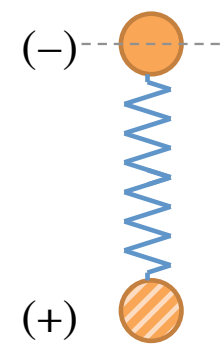

No field

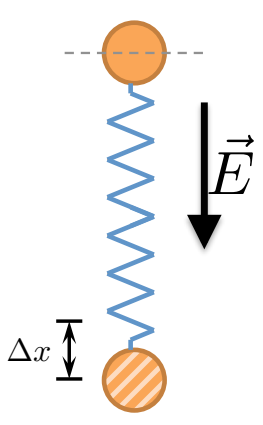

Parallel field:

Bond dilation

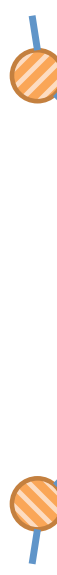

(b)

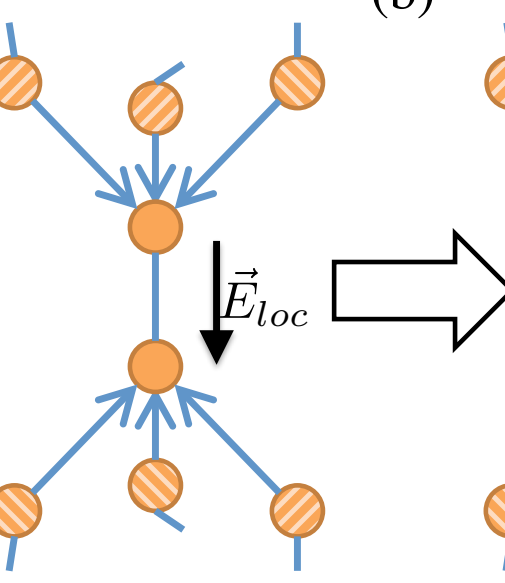

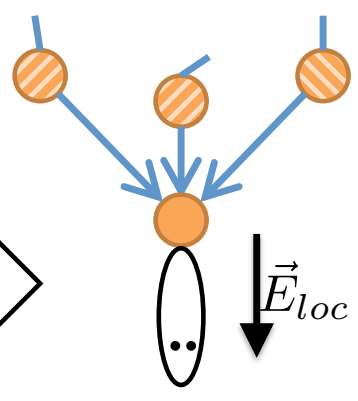

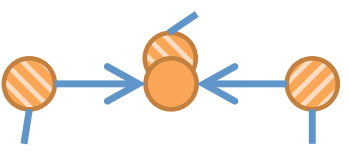

Figure 2.16: Schematics of the bond changes before (a) and after (b) the dielectric breakdown process in $\mathrm{SiO}_{2}$ [224].

temperature, $E$ is the electric field, and $\gamma$ is a field acceleration parameter that is dependent on a number of physical aspects of the system including bonding, defect concentration, and temperature.

McPherson details this energetic process well [224]. Physically, under an applied electric field, a polarization will occur and there will be an induced dipole between atoms that will result in strained bonds. As demonstrated in Fig. 2.16a, if the dipole is oriented parallel to the electric field this will stretch the associated bonds. This effect has been shown to be as large as a $2 \%$ strain in $\mathrm{SiO}_{2}$, which in comparison to the $7 \%$ strain associated with bond breakage is quite large [224]. The straining of these bonds leads to an overall reduction in the amount of thermal energy needed to break the bonds (given at zero field to be the activation energy $\Delta H_{0}$ in Eq. 47 ). 
When the strained dipoles under the electric field actually separate (i.e., when the thermal energy in the system exceeds the activation enthalpy for bond breakage), dielectric breakdown is said to occur. This process, shown for $\mathrm{SiO}_{2}$ in Fig. 2.16b, leads to a loss of the electrically insulative state normally seen in dielectrics and ultimately failure of the associated device.

The atomic-scale effects of thermal energy in this process come into play in terms of phonon interactions with polarized dipoles [215]. If atoms in a dipole already have a strained bond, interaction with a phonon may impart a high enough perturbation to break the bond. Energetically, the phononstrained bond interaction brings the system to a state where it is in a lower energy state if the local molecule is separated. Bond separation can lead to mobile electrons and point defects that alter the conductivity of the material. The presence of point defects in this system will not only change the energetic state of the local molecules, but may lead to a shift in local phonon frequencies that could severely impact the threshold to dielectric breakdown.

Further advances in characterization have led to recent studies that utilized techniques such as atomic force microscopy, scanning electron microscopy, transmission electron microscopy, scanning tunneling, and even ultra-fast lasers to characterize the breakdown process [197, 225-228]. There have also been recent advances in the modeling of dielectric breakdown [216, 229, 230] that, along with the experimental characterization, point towards a filament driven breakdown process. This process is essentialy an extension of the "tree" characteristics that were used to characterize breakdown decades before, but extend our understanding by identifying the mechanisms behind the conductive paths that would enable dielectric breakdown. Many of these studies point directly to defects in dielectric materials as the source of filament formation and development [197, 231].

Using advanced nanoscale thermal characterization techniques, such as TDTR, we will be able to identify the phonon-defect interactions and help to further the base of understanding that goes into addressing the issue of dielectric breakdown in functional oxides. 


\section{$3 \quad$ Measurement of Nanoscale Thermal Transport}

In order to measure thermal transport on length scales in the nanometer to micrometer range, and thermal carriers with scattering times of picoseconds, we must turn to ultra-sensitive techniques such as Time Domain Thermoreflectance (TDTR) [24-26] and/or Frequency Domain Thermoreflectance (FDTR) $[232,233]$. These optical pump-probe measurement techniques rely on a frequency modulated heating event that is sampled by an unmodulated probe beam. A typical TDTR sample consists of a material of interest coated with a thin (nominally $80 \mathrm{~nm}$ ) metal transducer that converts the modulated optical pump beam into a modulated thermal event penetrating into the sample. A component of the previously unmodulated probe beam, if aligned with the pump, will then be modulated at the prescribed frequency due to changes in reflectivity with temperature [234].

Using a lock-in amplifier, we are able to isolate the frequency component of the reflected probe beam and accurately measure the changes in thermoreflectance at the surface of the sample from the modulated heating event, penetrating 10's of nm to micrometers into the sample (depending on the material).

\subsection{Frequency Domain Thermoreflectance}

Both TDTR and FDTR utilize a frequency modulated optical pump beam for the thermal excitation. In FDTR, the pump and probe beams can be either pulsed or continuous wave (CW), allowing for a variety of experimental configurations, all of which fall under the FDTR technique. We will first address FDTR, since frequency modulated heating is common to both techniques and is easier conceptualized without pulsed sampling.

Frequency modulated heating for thermoreflectance characterization is the key component to

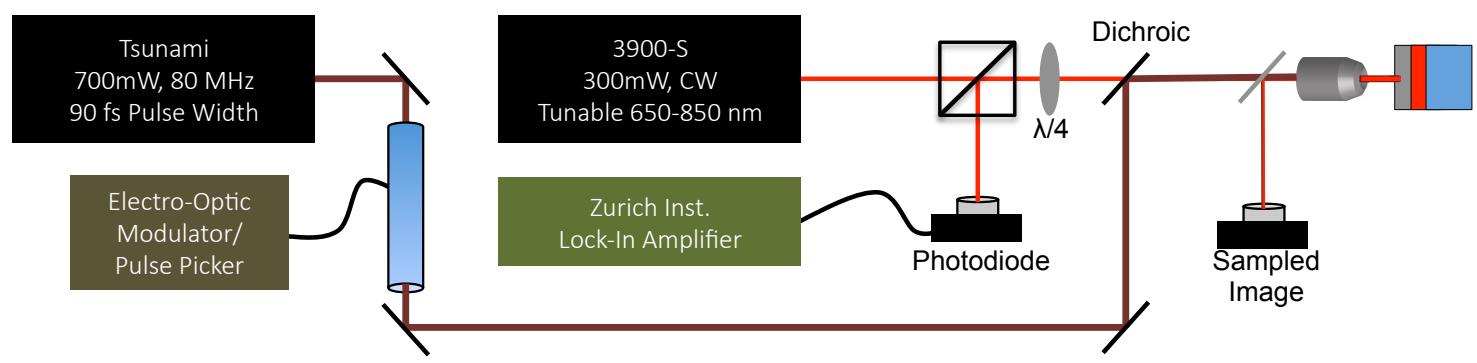

Figure 3.1: Schematic of a pulse-CW Frequency Domain Thermoreflectance system. 
these multilayer nanoscale measurement techniques. One reason for this is the ability to use lockin detection to isolate the frequency component of the probe that samples the temperature of the surface of the multilayer material. Changes in the reflectivity of a material with temperature are on the order of $1 \times 10^{-5}-2 \times 10^{-4}$ [234], so without the amplification of the signal from lock-in detection, we would not be able to detect the thermal response.

Additionally, the modulated heating event establishes a steady state control volume through the thickness of the sample, which we take as the sampled depth into the multilayer stack. In our modeling and sensitivity analysis, discussed in later sections, our visibility into the system is limited by this thermal penetration depth.

In comparison, an unmodulated beam simply heats the entire sample and will continue to do so until it reaches a steady state with the ambient temperature, and a single shot of a heating event will decay into the sample rather quickly (depending on the thermo-physical properties of the sample). For frequency modulated heating, for which we utilize either sinusoidal modulation or modulation by a square wave (on/off type of modulation), the situation results in a controlled thermal penetration depth. As mentioned, this depth defines the physical volume of which our system samples. With frequency modulated heating of a pulsed-pump, we actually have a combination of all of these effects. We will discuss various physical aspects of the resulting signal in later sections, but depending on the component of the resulting signal, we can isolate the single shot characteristics from the pulse, the steady state temperature rise from the continuous input of energy, or the accumulation of the pulsed heating separately.

In terms of on/off time of the heating, it follows that the lower the modulation frequency, the longer the heater is on, and the deeper into the sample the thermal gradient will reach. Quantitatively, this can be estimated as

$$
d_{\text {therm }}=\sqrt{\frac{\kappa}{\pi C_{v} f_{\text {mod }}}}
$$

where $\kappa$ and $C_{v}$ are the thermal conductivity and volumetric heat capacity of the layer of interest, respectively, and $f_{m o d}$ is the heating modulation frequency. To give an idea of orders of magnitude, for a pure silicon substrate (assuming $\kappa=145 \mathrm{~W} \mathrm{~m}^{-1} \mathrm{~K}^{-1}$ and $C_{v}=1.6 \mathrm{MJ} \mathrm{m}^{-3} \mathrm{~K}^{-1}$ ) and an 8.8 $\mathrm{MHz}$ modulation frequency, the thermal penetration depth will be approximately $1.8 \mu \mathrm{m}$. In 


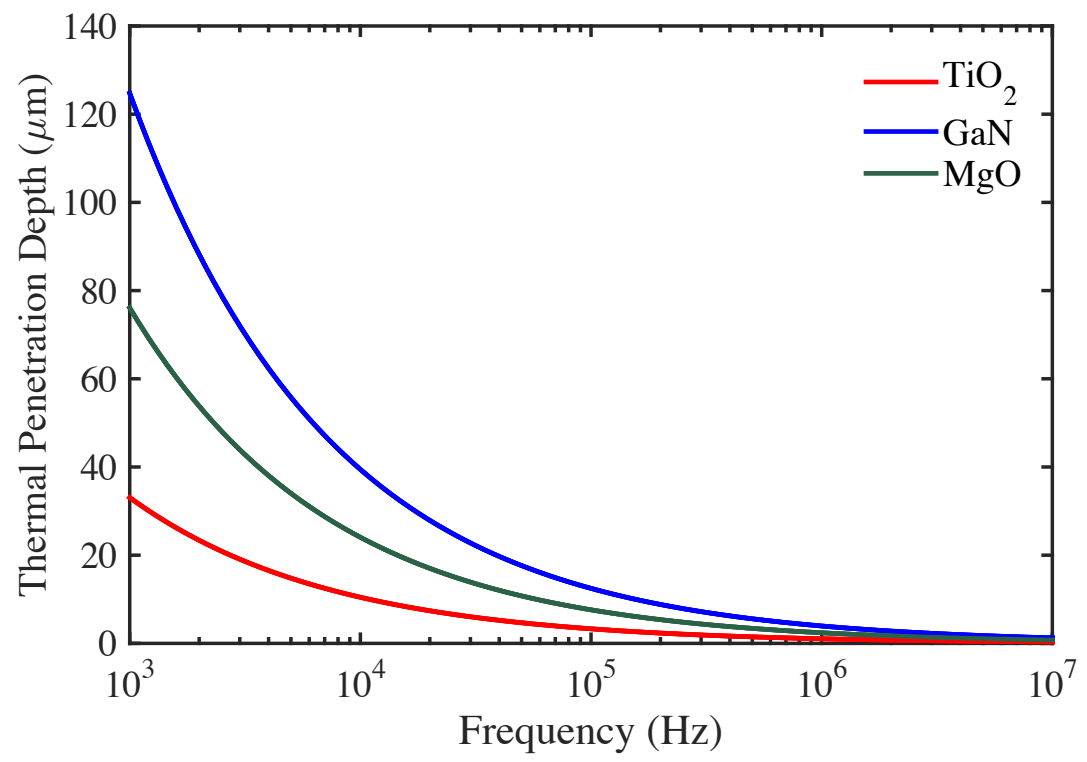

Figure 3.2: Dependence of thermal penetration depth for a modulated heating event on modulation frequency for $\mathrm{TiO}_{2}$ (red, $\kappa \approx 7 \mathrm{~W} \mathrm{~m}^{-1} \mathrm{~K}^{-1}$ ), $\mathrm{MgO}\left(\right.$ green, $\kappa \approx 60 \mathrm{~W} \mathrm{~m}^{-1} \mathrm{~K}^{-1}$ ), and GaN (blue, $\kappa \approx 160 \mathrm{~W} \mathrm{~m}^{-1} \mathrm{~K}^{-1}$ )

less thermally conductive materials, this depth will be significantly less $\left(\sim 375 \mathrm{~nm}\right.$ in $\left.\mathrm{TiO}_{2}\right)$ and can dictate the ability to measure effects of interfaces other than the interface between the transducer and the top layer. The change in thermal penetration depth with respect to modulation frequency corresponds to a relatively wide range of sampling volumes. Seen in Fig. 3.2, for a frequency sweep between $\mathrm{kHz}$ and $\mathrm{MHz}$ (which is not uncommon in an FDTR scan) the sampled volume can change from 100's of $\mu \mathrm{m}$ to 100's of nm. Often this change in depth is exploited to account for resulting sensitivity shifts associated with this change in sampling volume, such as fitting in-plane versus cross-plane thermal conductivity in the different penetration depth regimes.

Fig. 3.1 shows a schematic of an FDTR system, which, in this example is a pulsed pump-CW probe FDTR. We see the crucial components in this example are the pump beam, probe beam, electrooptic modulator, and lock-in amplifier. The data that is collected during an FDTR scan consists of the modulation frequency, the magnitude of the thermoreflectance signal, and the phase of the thermoreflectance signal. In addition to these parameters, we also collect the magnitude of the signal when the pump beam is blocked in order to record and subtract the noise floor during postprocessing. The most common signal analyzed in FDTR data in the literature is the phase offset, or the difference in phase of the frequency modulated pump and the thermoreflected probe. In order to determine this offset, and to correct the other parameters that may be of interest for analysis, 
we must first determine the shift in phase at each frequency due to the electronics. To do this, we follow the method outlined by Yang et al. where the pump is reflected directly into the detector (with the filters removed) and an FDTR scan is executed [235]. The strictly electronic phase shift is then used to normalize other data using the same scan parameters to determine the real shift in phase and correct the other signal components. It is crucial to mention here that all electronics stay constant between the reference scan and the scan of interest, even a change of cording can alter the electronic phase shift in your system.

In order to extract materials properties from an FDTR or a TDTR measurement, we must be able to model the frequency-dependent heating response of the system. In Sect. 3.4 we delve into the analysis process which takes into account the thermo-physical properties of a multilayer system that is undergoing a modulated heating event.

Opportunity for Further Investigation: The FDTR configuration featured in Fig. 3.1 is one that has been specifically built for some interesting potential experiments. Utilizing the pulsed-pump with the CW probe, it is theoretically possible to reconstruct the time domain signal of the pulsed heating event by analyzing the pulse frequency component of the CW probe. With a large enough frequency bandwidth on a lock-in detector along with a boxcar averaging system that can recreate the pulse window, one should be able to resolve the thermoreflectance decay between pulses.

\subsection{Time Domain Thermoreflectance}

While we can use FDTR to determine the frequency-dependent thermal response of a multi-layer system, adding in the ability to monitor the temporal response offers some distinct advantages. We use time domain thermoreflectance (TDTR) to elucidate information about the thermal carriers in a system over characteristic times ranging from picoseconds to nanoseconds. Utilizing the time component of the thermal response of a system allows us to glean information about electron-phonon dynamics, acoustic strain wave propagation, thermal conductance across boundaries, and thermal conductivity within various layers at spatial resolutions on the order of nanometers all in one scan, an example of which is shown in Fig. 3.3. In addition, TDTR offers a direct method to eliminate systematic electronic noise from gathered data as well as the ability to use advanced optics to more 
easily isolate the pump and probe (broad enough spectrum for wavelength separation, pulsed beam for frequency doubling, etc.).

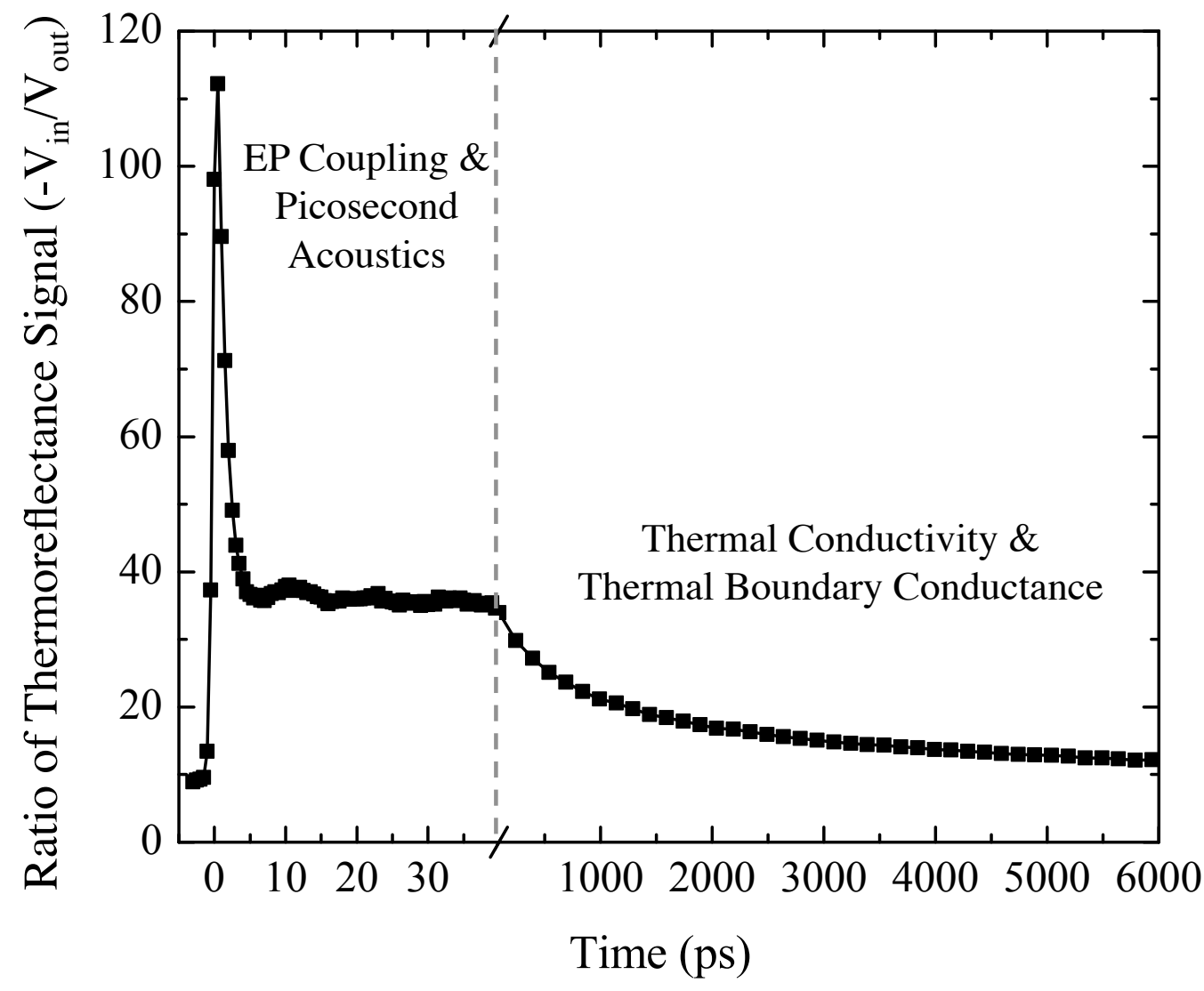

Figure 3.3: A representative Time Domain Thermoreflectance (TDTR) scan for $30 \mathrm{~nm}$ of Au on a glass substrate. The ratio of the in-phase and out-of-phase signals collected by the lock-in amplifier are plotted vs. pump-probe delay time.

Laid out in Fig. 3.4, TDTR is based around a train of ultra-fast laser pulses to thermally stimulate a material system, and a time delayed probe pulse train to measure the change in thermoreflectance due to the decay of the thermal energy deposited by the pump pulse. In our measurements, we utilize sub-picosecond laser pulses emanating from a Ti:Sapph. laser system at $80 \mathrm{MHz}$. A sample of interest is irradiated with a train of modulated pump pulses, while the thermoreflectance of the time delayed probe pulses is monitored for up to $6.5 \mathrm{~ns}$ after the pump heating event on the sample surface.

This is accomplished by splitting the pulse train emanating from the oscillator into two separate beam paths, these paths are generally split such that the pump path has significantly higher power than the probe path, since the pump will be inducing the heating event, and the probe will be measuring said event and ideally not heating the sample significantly. The pump then travels through 


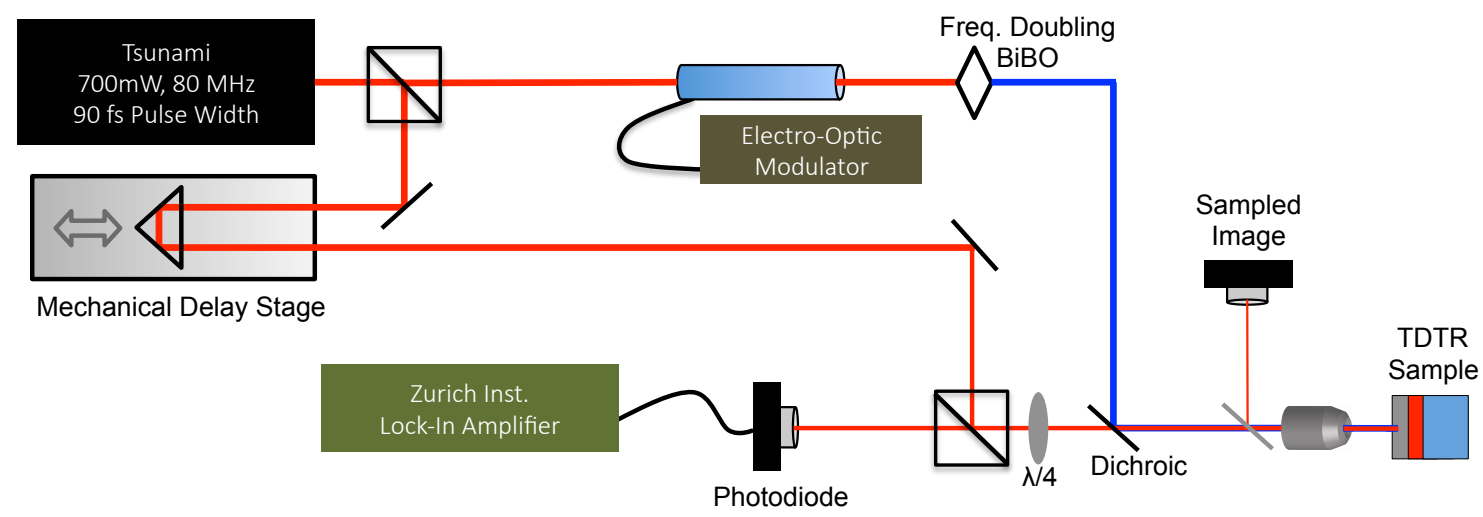

Figure 3.4: Schematic of a two-color Time Domain Thermoreflectance system.

an electro-optic modulator [236]. This is a Pockels cell which is driven at a prescribed frequency, and amplitude modulates the beam with either a square wave or a sine wave modulation, dictated by an external signal generator. In the system described here, the pump is then focused through a second harmonic generating crystal made of $\mathrm{BiB}_{3} \mathrm{O}_{6}$ where a two-photon excitation event occurs doubling the frequency of the emitted light and converting it from light with an $800 \mathrm{~nm}$ wavelength to a 400 nm wavelength [237].

We then steer the beam through a beam compressor (not shown in Fig. 3.4) before it is joined co-linearly with the probe beam using a dichroic mirror. A dichroic mirror is an optical component that is designed to either reflect or transmit light based on wavelength; in the case of this dichroic, $400 \mathrm{~nm}$ light is reflected while $800 \mathrm{~nm}$ light is transmitted; this is known as a cold mirror. The pump is then focused onto the sample using an objective lens and it is this focused spot that creates the modulated heating event (at the frequency prescribed by the input to the electro-optic modulator).

From the separation of the pump and probe, the probe travels first to a mechanical delay stage. This delay stage is a set of translated reflecting mirrors that physically change the path length that the probe travels before it arrives at the sample surface. Doing this enables us to change the time that the pulses from the probe are sampling the pulsed, modulated heating event. After the probe travels through the delay stage it is directed to a polarizing beam cube where the S-polarized light is reflected towards the objective to be joined with the pump. Before joining the pump, the probe passes through a $\lambda / 4$ wave-plate where the polarization is rotated such that, upon being reflected and passing through the $\lambda / 4$ wave-plate a second time, the now P-polarized probe passes through the polarizing beam cube. The probe beam, once co-linear with the pump, goes through the objective 
and is focused at the center of the pump spot (heating event). Since the alignment of the probe with respect to the detection optics must stay static, we use the cold mirror to steer the pump beam and monitor the thermoreflectance signal to ensure true overlap of the pump and probe at the sample surface.

After the probe has arrived on the sample surface, it is reflected back down the objective to be collected (the magnitude of this reflectance is dictated in part by the temperature of the sample surface, thus the pump heating event). We can use a beam sampler along this path to steer a component of this beam into a CCD camera to monitor the surface of the sample and to ensure that the probe is focused as tightly as possible. The reflected probe, carrying information about the modulated heating event that it has sampled is ultimately collected by a photodiode that sends a response to a lock-in amplifier. In this experimental setup the photodiode is a simple Si-based detector purchased from ThorLabs (DET10A) and the lock-in detector is from Zurich Instruments (HF2LI). Depending on the noise levels in the system, a number of filters, amplifiers, and chokes can be employed between the photodetector and the lock-in in order to ensure a high signal-to-noise ratio. In addition, despite the majority of the pump being reflected away from the returning probe path by the cold mirror, there is an optical filter (in this case, based on color) that ensures that no pump light arrives at the photodetector. An easy experimental check for this "pump bleed-through" lies in comparing the magnitude of the signal in the lock-in detector when both beams are blocked, when the pump only is blocked, and when the probe only is blocked. If there is no pump light leaking into the photodetector, all of these scenarios should result in the same low level of background noise.

The DC signal going into the photodetector should be used to determine the final alignment of the reflected probe beam and the actual photodiode; for a constant probe power, this value should be maximized based on alignment of the probe focused into the detector to ensure the best possible signal. Once the signal arrives at the lock-in amplifier, it will be compared to a reference signal that is fed in from the signal generator driving the electro-optic modulator and the frequency component of the probe that corresponds to the modulation frequency will be detected. At each pump-probe delay time and frequency, this signal will have a certain amplitude and phase offset from the reference signal. These two pieces of information are collected at each pump-probe delay time in order to form the TDTR decay curve shown in Fig. 3.3.

The mathematical analysis of this signal, as it relates to the physical modulated heating event 
and thermal properties of a multi-layer material will be addressed in Sect. 3.4. However, in order to deconvolve the physical phase offset of the heating event (which contains a substantial amount of information about the thermal dynamics of the sample) from the electronic detection phase offset, we must correct this signal using the following method.

The in-phase, $X$, and out-of-phase, $Y$, signals can be determined using the detected amplitude, $R$, and phase, $\phi$ of the lock in amplifier where

$$
\begin{gathered}
R \exp (i \phi)=X+i Y=R \cos \phi+i R \sin \phi \\
X=R \cos \phi \\
Y=R \sin \phi
\end{gathered}
$$

and the ratio of the in-phase and out-of-phase signals, or $-X / Y$, are generally analyzed in a TDTR experiment. The output signals from the lock-in amplifier need to be modified to account for the added phase offset before taking the ratio of these components. In FDTR, this is done by referencing the pump beam directly and taking extra data [235]. In TDTR, we can utilize the known nature of the out-of-phase signal in order to determine the phase offset. As the pump-probe delay time passes through $t_{0}$, or the time at which both the pump and probe arrive at the sample simultaneously, the $Y$ signal should not change $[25,26]$. This is due to the fact that the $Y$ signal is dominated by the response from thermal accumulation between pulses and not significantly from the heating of individual pulses (which is captured largely in the $X$ signal). Knowing this, we can assign any change in the "uncorrected" $Y$ signal to the phase offset from the electronics and thus determine the value of this phase offset and correct the respective signals. Along these lines, it is good experimental practice to manually alter the phase offset at the lock-in amplifier to minimize the shift in $Y$ through $t_{0}$. This process allows you to gain an understanding of the "real" $Y$ signal as a corollary to the amount of thermal accumulation on the sample based on pump-frequency and powers. After recording this, best practice dictates manually adjusting the additional phase offset so that there is a significant "jump" in the $Y$ signal through $t_{0}$ before starting a TDTR scan. This jump increases ease of identification 
of the phase offset in post-processing, allowing us to be sure that we have accounted for alterations to our signal from the electronics in the detection.

We find the phase offset by subtracting the detected $Y$ signal before $t_{0}$ from the $Y$ signal immediately after $t_{0}$.

$$
\begin{gathered}
\phi_{\text {elec }}=-\tan ^{-1}\left(\frac{\Delta Y}{\Delta X}\right) \\
\Delta Y=\left|Y\left(t<t_{0}\right)-Y\left(t>t_{0}\right)\right| \\
\Delta X=\left|X\left(t<t_{0}\right)-X\left(t>t_{0}\right)\right|
\end{gathered}
$$

where $\phi_{\text {elec }}$ is the offset phase from the electronics. When this has been determined, the correction can be applied to the $X$ and $Y$ signals in order to be used for the subsequent analysis.

$$
\begin{gathered}
X_{\text {corr }}=X_{o} \cos \phi_{\text {elec }}-Y_{o} \sin \phi_{\text {elec }} \\
Y_{\text {corr }}=Y_{o} \cos \phi_{\text {elec }}+X_{o} \sin \phi_{\text {elec }}
\end{gathered}
$$

where $X_{o}$ and $Y_{o}$ are the $X$ and $Y$ signals before the correction and $X_{\text {corr }}$ and $Y_{\text {corr }}$ are the corrected signals to be used in the analysis. Geometrically, this process can be thought of in terms of the unit circle, shown in Fig. 3.5, where the signal vector with magnitude, $R$, given by the lock-in amplitude and angle, $\phi$, given by the lock-in phase are interchangeably defined by the in-phase, $X$, and out-ofphase, $Y$, signals. The mathematical treatment in Eq.'s 55 and 56 is equivalent to rotation of the signal vector by an angle corresponding to the induced phase offset by the detection electronics.

After applying these corrections, you can then use these values of the magnitude, corrected phase, in-phase, and out-of-phase signals to analyze a TDTR decay curve along with the known model for the thermal response of a multilayered system and determine any unknown quantities such as the thermal conductivity of a layer or the thermal boundary conductance between two layers.

We can also use TDTR data to glean thermophysical information beyond the thermal conductivity 


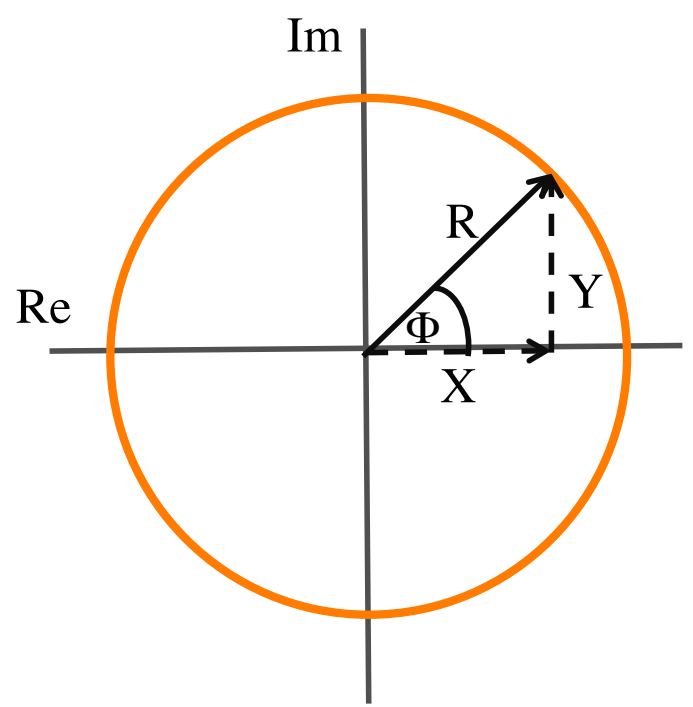

Figure 3.5: The unit circle relating the amplitude and phase of the detected TDTR signal to the in-phase and out-ofphase components. Phase correction is done, geometrically, by rotating this vector by an amount corresponding to the phase offset induced by the detection electronics.

and the thermal boundary conductance of a material, which is normally done by fitting the decay at pump-probe time delays in the nanosecond range. The thermoreflectance signal in early pump-probe delay times (in the picosecond range) can be useful in measuring fast dynamics such as electronphonon coupling and acoustic strain wave propagation.

Electron-phonon coupling is a field in-and-of-itself, with applications ranging from basic electronics to next generation alternative energy sources, characterization challenges that entice experimentalists all over the world, and theoretical understanding that even still allows for significant advances in fundamental understanding of these transport mechanisms [238-245]. My experience with electron-phonon coupling has been peripheral, but TDTR and other femtosecond pump-probe techniques are widely used to study these phenomena as they occur on timescales in the femtosecond to single picosecond regime. Electron phonon coupling analysis involves investigation of the magnitude and characteristics of the thermoreflectance peak at and around $t_{0}$, when the electrons in the system have just absorbed the energy from the optical pump and are still out of equilibrium with the phonons [239].

Another very useful aspect of the early time component of the TDTR scan occurs in the 10's of picoseconds range, and that is acoustic strain wave propagation. As can be seen in Fig. 3.3, effects of a resonating strain wave appear in the TDTR scan in the form of perturbations of the decay of the 
thermoreflectance signal. These perturbations result from an initial expansion of the lattice during the first stages of the pump absorbance that propagates at the speed of sound through the film and, depending on the mechanical properties of the film and the neighboring layer, will reflect off of the interface and change the film reflectance upon returning to the surface that the laser is sampling [246, 247]. This effect, referred to often as picosecond ultrasonics [248] or picosecond acoustics [249], can be used to determine mechanical and bonding properties of interfaces, electron diffusion dynamics, acoustic characterization of multi-phase interfaces, and the list goes on [250-258]. The most common use in our research group is a verification of metal transducer film thickness [249]. With known speed of sound, the time it takes to see a perturbation in the thermoreflectance signal compared to pulse absorption time can be used to determine the thickness of the film being interrogated, since that is the distance the strain wave must travel. This process has been employed in each experimental work described in this dissertation. As long as there is a mismatch in acoustic impedance of the film and the interface, and the film is thin enough so as to not damp out any oscillations that are present, you can determine this sort of information using the short time information of a TDTR scan.

\subsection{Thermal Transport in a Layered Structure with a Modulated Source}

The response of the thermoreflectance signal can be fit to a thermal model to determine the physical properties that dictate how heat will escape the control volume. The foundation of the thermal model that we use for one dimensional conduction in a layered structure follows the method of Carlslaw and Jaeger [259]. In this model, we determine the heat flux and temperature on either side of any given layer by the conditions at each layer's boundaries. Ultimately the system is defined by the boundary conditions prescribed by the optical heating event at the surface of the top layer and an assumed semi-infinite boundary condition at the back of the bottom layer, or substrate.

Generally in this analysis, when using a laser spot as a heating source, a three dimensional, cylindrically symmetric thermal diffusion condition is the most physically accurate representation of the Gaussian beam. However, by using a relatively large spot size for the pump and relatively high modulation frequencies, we stay in the regime of probing strictly one dimensional thermal diffusion in the cross-plane direction. In each experiment shown in this dissertation, the pump spot is larger than $30 \mu \mathrm{m}$ in diameter and the probe is $20 \mu \mathrm{m}$ or smaller, in this case the probe is essentially sampling a radially constant heating event and is not sensitive to any of the thermal diffusion towards the 
outside edge of the pump.

Modulation frequency can also dictate sensitivity to the in-plane dimension of the thermal decay. At high modulation frequencies the shallow depth of the thermal gradient compared to the radius of the heating event result in one-dimensional diffusion throughout the thickness of the probed region. At low modulation frequencies, however, the thermal penetration depth can reach similar orders of magnitude as the radius of the pump and the thermal decay in the radial direction must be taken into account. Again, the experiments presented in this dissertation all use modulation frequencies which maintain one-dimensionally dominated thermal diffusion in the cross-plane direction, ranging from $1 \mathrm{MHz}-8.8 \mathrm{MHz}$.

An easy check for the validity of the one-dimensional solution is a sensitivity analysis of a representative multilayer structure at various spot sizes, comparing the sensitivity to the cross-plane and in-plane thermal conductivities. The process behind the sensitivity analysis and full interpretation of the results of a sensitivity analysis are outlined in Ch. 3.5. Figure 3.6 shows the results of a sensitivity analysis for the $\mathrm{BaTiO}_{3}$ thin film measurement that will be shown in Ch. 4 . The key aspect of this measurement in this case is the relative magnitude of the sensitivity to each component of the thermophysical system.

The analysis conducted for Fig. 3.6 clearly demonstrates that using the spot sizes and modulation frequencies discussed, the assumption of one-dimensional thermal decay is valid for the studies in this dissertation. Since this is the case, and for ease of explanation, I will ignore radial thermal diffusion and discuss the formulation of the analysis of thermoreflectance decay for one-dimensional thermal diffusion only over the course of this chapter.

We start the derivation for this relationship with the one-dimensional heat equation without internal energy generation (the heat flux from the modulated pump will be treated as a boundary condition).

$$
\frac{\partial^{2} T}{\partial z^{2}}=\frac{C_{v}}{\kappa} \frac{\partial T}{\partial t}
$$

where the cross-plane thermal conductivity is $\kappa$, the volumetric heat capacity is $C_{v}, T$ is the temperature of the system, and $z$ and $t$ are depth and time, respectively. As mentioned, we assume one-dimension thermal transport and set boundary conditions assuming we know the temperature 


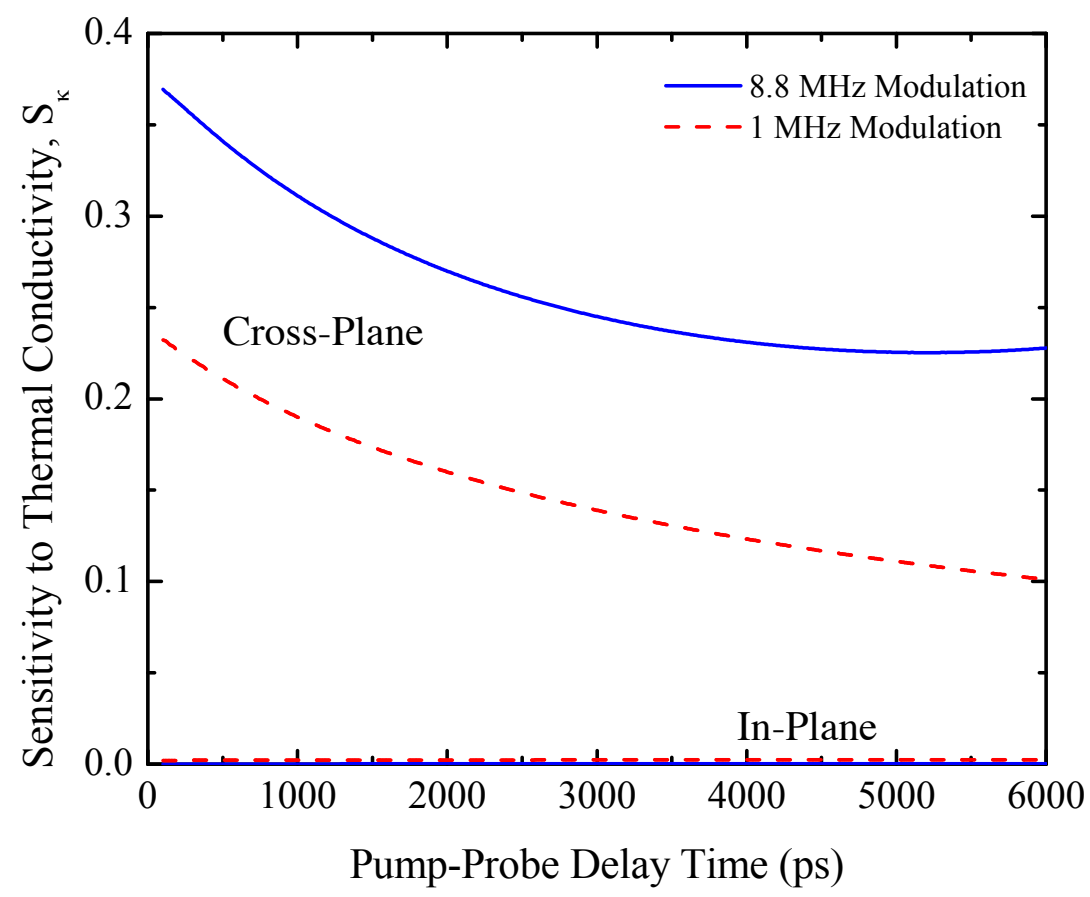

Figure 3.6: Sensitivity analysis of the cross-plane and in-plane thermal conductivity of $\mathrm{BaTiO}_{3}$ thin films with $30 \mu \mathrm{m}$ and $18 \mu \mathrm{m}$ pump and probe spot sizes, respectively, at two modulation frequencies $(8.8 \mathrm{MHz}$ and $1 \mathrm{MHz})$. In both cases, the sensitivity to the in-plane thermal conductivity is negligible and verifies an assumption of one-dimensional thermal diffusion in this TDTR experiment.

and heat flux at the top and bottom boundaries. The boundary conditions at the front face of the layer of interest are determined by the known temperature at the front, $T_{\text {front }}$, and back, $T_{\text {back }}$, of the layer.

$$
\begin{gathered}
@ z=0 \rightarrow T=T_{\text {front }} \\
@ z=d \rightarrow T=T_{\text {back }}
\end{gathered}
$$

where $d$ is the layer thickness. Other boundary conditions are dictated by the heat flux, $q$ at the front and back of the layer by Fourier's Law, $q=-\kappa \frac{d T}{d x}[260]$. 


$$
\begin{aligned}
& @ z=0 \rightarrow \frac{\partial T}{\partial z}=\frac{-q_{\text {front }}}{\kappa} \\
& @ z=d \rightarrow \frac{\partial T}{\partial z}=\frac{-q_{\text {back }}}{\kappa}
\end{aligned}
$$

since we will be dealing with a frequency-dependent heating event, we will transform the time dependent form of the temperature into frequency-space using a Fourier transform. In general,

$$
\mathbf{f}(\omega)=\mathcal{F}\{f(t)\}=\int_{-\infty}^{\infty} f(t) e^{-i \omega t} d t
$$

where $\mathbf{f}$ is the Fourier transform of the arbitrary function, $f, \omega$ is frequency and $t$ is time. In addition, we know that

$$
\frac{d f(t)}{d t}=i \omega \mathbf{f}(\omega)
$$

thus Eq. 57 becomes

$$
\frac{\partial^{2} \mathbf{T}}{\partial z^{2}}=\frac{i \omega C_{v}}{\kappa} \mathbf{T}(\omega)
$$

where $\mathbf{T}$ is the fourier transform of $T$, which is now a function of frequency and depth instead of time and depth. We can substitute in the thermal diffusivity, $\alpha=\frac{\kappa}{C_{v}}$ and rearrange.

$$
\frac{\partial^{2} \mathbf{T}}{\partial z^{2}}-\frac{i \omega}{\alpha} \mathbf{T}=0
$$

which is a differential equation with solutions in the form of

$$
\mathbf{T}(z, \omega)=C_{1} \cosh \left(\sqrt{\frac{i \omega}{\alpha}} z\right)+C_{2} \sinh \left(\sqrt{\frac{i \omega}{\alpha}} z\right)
$$

revisiting the boundary conditions, we will consolidate variables since we are only concerned with dependance on the depth, $z$. We will refer to $\mathbf{T}(z, \omega)$ as $\mathbf{T}(z)$ and $\sqrt{\frac{i \omega}{\alpha}}$ as $b$. 


$$
\begin{aligned}
@ z=0 & \rightarrow \mathbf{T}(0)=T_{\text {front }} \\
& \rightarrow \mathbf{T}(0)=C_{1}=T_{\text {front }} \\
@ z=0 & \rightarrow \frac{\partial \mathbf{T}(0)}{\partial z}=\frac{-q_{\text {front }}}{\kappa} \\
& \rightarrow \frac{\mathbf{T}(0)}{\partial z}=C_{2} b=\frac{-q_{\text {front }}}{\kappa} \\
@ z=d & \rightarrow T=T_{\text {back }} \\
& \rightarrow \mathbf{T}(d)=T_{\text {front }} \cosh (b d)-q_{\text {front }} \frac{\sinh (b d)}{b \kappa}=T_{\text {back }} \\
@ z=d & \rightarrow \frac{\partial \mathbf{T}(d)}{\partial z}=\frac{-q_{\text {back }}}{\kappa} \\
& \rightarrow \frac{\mathbf{T}(d)}{\partial z}=T_{\text {front }} b \sinh (b d)-q_{\text {front }} \frac{\cosh (b d)}{\kappa}=\frac{-q_{b a c k}}{\kappa}
\end{aligned}
$$

this results in the following relationship between the temperatures and heat fluxes on either side of the layer.

$$
\begin{aligned}
& T_{\text {back }}=T_{\text {front }} \cosh (b d)-q_{\text {front }} \frac{\sinh (b d)}{b \kappa} \\
& q_{\text {back }}=-T_{\text {front }} b \kappa \sinh (b d)+q_{\text {front }} \cosh (b d)
\end{aligned}
$$

which can be combined into a matrix for easier treatment. 


$$
\left[\begin{array}{c}
T_{b a c k} \\
q_{b a c k}
\end{array}\right]=\left[\begin{array}{cc}
\cosh (b d) & \frac{-\sinh (b d)}{\kappa b} \\
-\kappa b \sinh (b d) & \cosh (b d)
\end{array}\right]\left[\begin{array}{c}
T_{\text {front }} \\
q_{\text {front }}
\end{array}\right]
$$

through matrix multiplication, we can model a multilayer system and the thermal response with modulated laser heating. In order to determine the thermal boundary conductance (TBC), we add in layers that have no thickness or heat capacity between the finite layers. This simplifies to

$$
\left[\begin{array}{c}
T_{\text {back }} \\
q_{\text {back }}
\end{array}\right]=\left[\begin{array}{cc}
1 & -1 / G \\
0 & 1
\end{array}\right]\left[\begin{array}{c}
T_{\text {front }} \\
q_{\text {front }}
\end{array}\right]
$$

where $G$ is the thermal boundary conductance.

This model allows us to determine the TBC between any given layers as long as the thermal penetration depth of our measurement is large enough and the signal components being used are sensitive to the TBC.

With this formulation for the temperature and heat flux at each boundary of a layered system, we can employ the matrix multiplication of the layers of interest in order to obtain the form of the temperature response (which is directly related to the thermoreflectance signal) of the top layer. For the material system studied in Sect. 5, an $\mathrm{Al}$ film on a $\mathrm{CdO}$ film on a $\mathrm{MgO}$ substrate, the matrix multiplication would look as follows.

$$
\begin{gathered}
{\left[\begin{array}{c}
T_{\text {back }} \\
q_{\text {back }}
\end{array}\right]=\left[\mathcal{L}_{A l}\right]\left[\mathcal{B}_{A l / C d O}\right]\left[\mathcal{L}_{C d O}\right]\left[\mathcal{B}_{C d O / M g O}\right]\left[\mathcal{L}_{M g O}\right]\left[\begin{array}{c}
T_{\text {front }} \\
q_{\text {front }}
\end{array}\right]} \\
{\left[\begin{array}{c}
\left.\mathcal{L}_{A l}\right]= \\
-\kappa_{A l} b_{A l} \sinh \left(b_{A l} d_{A l}\right) \\
{\left[\mathcal{B}_{A l / C d O} \cosh \left(b_{A l} d_{A l}\right)\right.}
\end{array}\right]=\left[\begin{array}{cc}
1 & -1 / G_{A l / C d O} \\
0 & 1
\end{array}\right]}
\end{gathered}
$$

where subscripts denote either the layer, $\mathcal{L}$, or the boundary, $\mathcal{B}$. 
The matrix multiplication of each layer and boundary can be simplified in one large $2 \times 2$ equivalent matrix which represents the thermo-physical properties of the entire multi-layer system.

$$
[\mathcal{K}]=\left[\mathcal{L}_{A l}\right]\left[\mathcal{B}_{A l, C d O}\right]\left[\mathcal{L}_{C d O}\right]\left[\mathcal{B}_{C d O, M g O}\right]\left[\mathcal{L}_{M g O}\right]
$$

where $\mathcal{K}$ is the matrix entries in the representative system. Thus, from Eq. 82

$$
\left[\begin{array}{c}
T_{\text {back }} \\
q_{\text {back }}
\end{array}\right]=[\mathcal{K}]\left[\begin{array}{c}
T_{\text {front }} \\
q_{\text {front }}
\end{array}\right]
$$

Using a semi-infinite boundary condition on the back of the multilayer stack, we are able to determine that the back temperature, $T_{\text {back }}$ will be the ambient temperature, $T_{\infty}$, and there will be no heat flux, $q_{b a c k}$, out of the back side. This enables us to have unknowns only in the materials parameters of interest since the incident heat flux is given by our laser parameters and the temperature response at the top surface is monitored via thermoreflectance. If we consider a temperature offset, $\Theta=T-T_{\infty}$, then the semi-infinite boundary condition yields an equation that can be easily solved for the components of the total thermo-physical system.

$$
\begin{gathered}
{\left[\begin{array}{l}
\Theta_{\text {back }} \\
q_{\text {back }}
\end{array}\right]=\left[\begin{array}{l}
0 \\
0
\end{array}\right]=[\mathcal{K}]\left[\begin{array}{l}
\Theta_{\text {front }} \\
q_{\text {front }}
\end{array}\right]} \\
{\left[\begin{array}{l}
0 \\
0
\end{array}\right]=\left[\begin{array}{ll}
\mathcal{K}_{11} & \mathcal{K}_{12} \\
\mathcal{K}_{21} & \mathcal{K}_{22}
\end{array}\right]\left[\begin{array}{l}
\Theta_{\text {front }} \\
q_{\text {front }}
\end{array}\right]} \\
\Theta_{\text {front }}=\frac{-\mathcal{K}_{22}}{\mathcal{K}_{21}} q_{\text {front }}
\end{gathered}
$$

this functional form can then be used to determine any of the parameters contained in the total thermo-physical system, as long as the other parameters are known. As a reminder these include the volumetric heat capacity, thermal conductivity, and thickness of each layer, as well as the thermal boundary conductance between layers. Of course, this determination is limited by sensitivity of the measurement to these parameters, a concept which will be discussed in Sect. 3.5. 


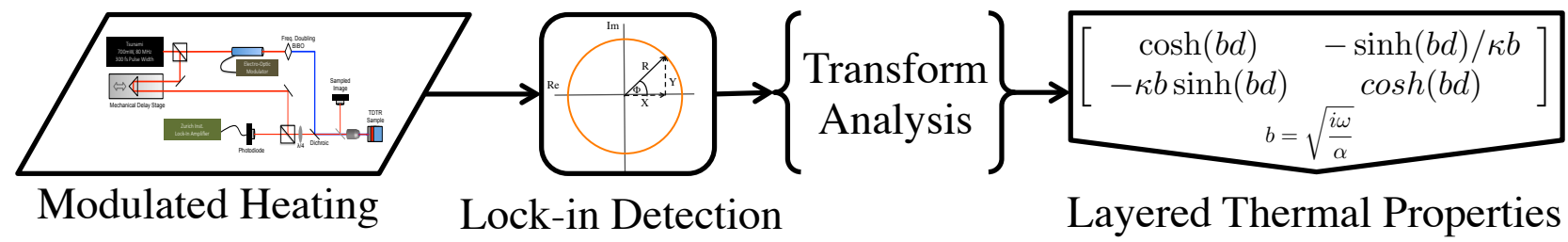

Figure 3.7: Flowchart of the progress of information from the physically sampled heating event, to the lock-in detection, through a transform analysis, ultimately to determine the thermo-physical parameters of a multi-layered system.

As mentioned, this analysis is derived for one-dimensional thermal diffusion, the major difference when taking into account radial thermal diffusion is the $b$ parameter, which would take into account separate thermal conductivity in the radial, or in-plane, direction. In the next part of this chapter, I will go on to use the result found in Eq. 89, keeping in mind a change in the $b$ parameter for radial thermal diffusion, to discuss the general relationship between the thermo-physical parameters and the other aspects of the thermoreflectance signal. This is mathematically valid since the functional form of Eq. 89 holds for radial transport and, if departing from the realm of one dimensional heat transfer, the only change is in the formulation of $\mathcal{K}_{22}$ and $\mathcal{K}_{21}$.

\subsection{Analysis of Lock-in Detection of a Thermoreflectance Signal}

With respect to determining the thermal properties of a multi-layer material system using a modulated thermoreflectance response, we have discussed the nature of the TDTR signal in Sect. 3.2, and the thermo-physical system in Sect. 3.3, but are missing the critical step of the transformation between a thermoreflectance signal and the actual physical system.

The flow of information laid out in Fig. 3.7 shows that this final step in the analysis will bridge the gap between the signal coming in to the detector and the relation to the thermal parameters $T_{\text {front }}$ and $q_{\text {front }}$ discussed in Eq. 89 in Sect. 3.3.

With the size and the incident power of the pump spot (measured by a beam profiler and power meter), we can determine the parameter $q_{\text {front }}$. The incident heating event from the pump absorption will be

$$
q_{\text {front }}=\frac{2 E_{0}}{\pi R_{0}^{2}} \exp \left(\frac{2 r^{2}}{R_{0}^{2}}\right) g(t)
$$

where $E_{0}$ is the absorbed energy from the pump, $R_{0}$ is the pump $1 / \mathrm{e}^{2}$ radius, and the function $g(t)$ 
describes the input of the pump in time. In the case of the physical description of the beam, I leave the formulation in terms of a radial profile, while the end result of this derivation will still yield a valid one-dimensional thermal transport assumption, the process of the derivation is incomplete without taking into account the beam shape. We can take the fourier transform of this function in order to convert the time-dependent nature of the pump into the frequency domain, a shift which will be useful when treating the frequency-dependent response in the FDTR and TDTR signals.

$$
\mathcal{F}\left\{q_{\text {front }}\right\}=\mathbf{q}_{\text {front }}=\frac{2 E_{0}}{\pi R_{0}^{2}} \exp \left(\frac{2 r^{2}}{R_{0}^{2}}\right) \mathbf{g}(\omega)
$$

where $\mathbf{q}_{\text {front }}$ is a function of both the pump radius and the pump frequency, $\omega$. Plugging this into Eq. 89 we see that

$$
\mathbf{T}_{\text {front }}(r, \omega)=\frac{-\mathcal{K}_{22}}{\mathcal{K}_{21}} \frac{2 E_{0}}{\pi R_{0}^{2}} \exp \left(\frac{2 r^{2}}{R_{0}^{2}}\right) \mathbf{g}(\omega)
$$

we now have a formulation for the surface temperature of the multilayer sample that depends on size and frequency of the pump heating event, as well as the thermo-physical parameters of the system. The resulting temperature will be sampled by the probe beam, which takes a form similar to the pump.

$$
\mathbf{q}_{\text {probe }}(r, \omega)=\frac{2}{\pi R_{1}^{2}} \exp \left(\frac{2 r^{2}}{R_{1}^{2}}\right) \mathbf{p}(\omega)
$$

where $\mathbf{q}_{\text {probe }}$ is the profile of the probe in space and frequency, $R_{1}$ is the $1 / \mathrm{e}^{2}$ radius of the beam, and $\mathbf{p}$ is the general frequency-domain form of the probe (which will be constant with a CW probe and depend on the pulse frequency in a pulsed probe). It should be noted here that the absorbed energy from the probe is not taken into account since this is assumed to be very low.

At a given temperature the reflectance of the top layer of the system will be dictated by the thermoreflectance coefficient, $\beta$, which is proportional to the change in temperature [261].

$$
\Delta R=\beta \Delta T
$$

where $R$ and $T$ are the reflectance and temperature, respectively. Thus, given the rise in temperature 
dictated by Eq. 92

$$
\Delta R_{\text {front }}=ß \mathbf{T}_{\text {front }}
$$

In transform space, the probe beam sampling of the surface temperature (dictated by the thermoreflectance) consists of a convolution of Eq. 93 and 95 (Ref. [262]).

$$
\begin{gathered}
\mathbf{q}_{\text {samp }}=ß \mathbf{T}_{\text {front }} * \mathbf{q}_{\text {probe }}=\beta \int_{-\infty}^{\infty} \mathbf{T}_{\text {front }}(r, \psi) \mathbf{q}_{\text {probe }}(r, \omega-\psi) d \psi \\
\mathbf{q}_{\text {samp }}=\beta \int_{-\infty}^{\infty} \frac{-\mathcal{K}_{22}}{\mathcal{K}_{21}} \frac{2 E_{0}}{\pi R_{0}^{2}} e^{\left(\frac{2 r^{2}}{R_{0}^{2}}\right)} \mathbf{g}(\psi) \frac{2}{\pi R_{1}^{2}} e^{\left(\frac{2 r^{2}}{R_{1}^{2}}\right)} \mathbf{p}(\omega-\psi) d \psi \\
\mathbf{q}_{\mathbf{s a m p}}=\beta \int_{-\infty}^{\infty} \mathfrak{R}(r) \frac{-\mathcal{K}_{22}}{\mathcal{K}_{21}} \mathbf{g}(\psi) \mathbf{p}(\omega-\psi) d \psi
\end{gathered}
$$

where $\mathbf{q}_{\text {samp }}$ is the probe beam after it has sampled the heating event and $\mathfrak{R}$ contains the radial dependence of the heating event and probe beams. This thermoreflected probe now contains the information about the modulated heating event that we need to collect using a photodetector and extract using the lock-in detector.

The collected probe beam will be converted into an electrical signal by the photodetector, which integrates the incident power over the space of the detector. In radial coordinates this corresponds to

$$
S_{\text {coll }}(\omega)=\int_{0}^{\infty} \mathbf{q}_{\text {samp }} 2 \pi r d r=\beta \int_{0}^{\infty} \int_{-\infty}^{\infty} \mathfrak{R}(r) \frac{-\mathcal{K}_{22}}{\mathcal{K}_{21}} \mathbf{g}(\psi) \mathbf{p}(\omega-\psi) d \psi 2 \pi r d r
$$

where $S_{\text {coll }}$ is the frequency dependent signal from the photodetector. We can reduce the problem to an integration only in frequency space by employing a treatment with zero order Hankel transforms. The zero order Hankel transform is a spatial transformation using a weighted sum of zero order Bessel functions, shifting from real space to inverse space much like Fourier transforms shift from time to inverse time (or frequency). 


$$
\tilde{f}(k)=\mathcal{H}_{0}\{f(r)\}=\int_{0}^{\infty} f(r) J_{0}(k r) r d r
$$

where $\tilde{f}(k)$ is the Hankel transform of an arbitrary function, $f(k), J_{0}$ is a zero order Bessel function, $r$ is a radial variable and $k$ is the transform variable in inverse distance. Applying this transform to the spatially dependent parameters of Eq. 100

$$
S_{\text {coll }}=\beta \int_{0}^{\infty} \int_{-\infty}^{\infty} \tilde{\mathfrak{R}} \frac{-\tilde{\mathcal{K}}_{22}}{\tilde{\mathcal{K}}_{21}} \mathbf{g}(\psi) \mathbf{p}(\omega-\psi) d \psi 2 \pi r d r
$$

where $\tilde{S}_{\text {coll }}$ is the $k$-dependent collected signal, $\tilde{\mathfrak{R}}$ is the $k$-dependent beam and heating profiles, and $\tilde{\mathcal{K}}_{22}$ and $\tilde{\mathcal{K}}_{22}$ are related to the thermo-physical parameters of the system which in general may depend on $\kappa$. Here the integration over these spatially dependent parameters is in itself a Hankel transformation, so grouping these parameters together we can reduce them to only an integration over $k$.

$$
\mathfrak{B}=\int_{0}^{\infty} \tilde{\mathfrak{R}} \frac{-\tilde{\mathcal{K}}_{22}}{\tilde{\mathcal{K}}_{21}} 2 \pi r d r=\frac{1}{2 \pi} \int_{0}^{\infty} \frac{-\tilde{\mathcal{K}}_{22}}{\tilde{\mathcal{K}}_{21}} e^{\left(\frac{k^{2}\left(R_{0}^{2}+R_{1}^{2}\right)}{8}\right)} d k
$$

where $\mathfrak{B}$ is a function only of frequency due to the frequency dependence of the thermo-physical parameters. Revisiting Eq. 102

$$
S_{\text {coll }}(\omega)=\beta \int_{-\infty}^{\infty} \mathfrak{B}(\psi) \mathbf{g}(\psi) \mathbf{p}(\omega-\psi) d \psi
$$

we now have the mathematical response of the photodetector that is sent to the lock-in amplifier and depends on the geometry of the beams, the temporal characteristics of the beams, and the thermo-physical parameters of the system. The final step is the detection of this signal by the lock-in amplifier.

In the general case with FDTR and TDTR, the pump will be modulated at a given frequency.

$$
\mathbf{g}(\omega)=\mathbf{g}_{o} \delta\left(\omega-\omega_{p}\right)
$$

where $\mathbf{g}_{o}$ is the frequency independent form of the pump and $\delta\left(\omega-\omega_{p}\right)$ is the frequency component of pump beam, modulated at a prescribed frequency combined with any other frequency components 
present. Adding this component to Eq. 104 we see that

$$
S_{\text {coll }}(\omega)=\beta \int_{-\infty}^{\infty} \mathfrak{B}(\psi) \mathbf{g}_{o} \delta\left(\psi-\omega_{p}\right) \mathbf{p}(\omega-\psi) d \psi
$$

with the property of the Dirac delta function

$$
\int_{-\infty}^{\infty} f(\omega) \delta\left(\omega-\omega_{o}\right) d \omega=f\left(\omega_{o}\right)
$$

we can determine that

$$
S_{\text {coll }}(\omega)=\beta \mathfrak{B}\left(\omega_{p}\right) \mathbf{g}_{o} \mathbf{p}\left(\omega-\omega_{p}\right)
$$

Now the probe can also be represented in terms of a product of its frequency dependent components

$$
\mathbf{p}\left(\omega-\omega_{p}\right)=\mathbf{p}_{o} \delta\left(\omega-\omega_{p}-\omega_{b}\right)
$$

where $\omega_{b}$ is a frequency dependent component of the probe that is separate from pump frequency. This general form leads to

$$
\begin{gathered}
S_{\text {coll }}(\omega)=\mathfrak{C}_{\text {gen }} \mathfrak{B}\left(\omega_{p}\right) \delta\left(\omega-\omega_{p}-\omega_{b}\right) \\
\mathfrak{C}_{\text {gen }}=ß \mathbf{g}_{o} \mathbf{p}_{o}
\end{gathered}
$$

where $\mathfrak{C}_{g e n}$ is a general constant dictated by the non-temporal factors in the system and the only frequency component aside from the Dirac delta function is the frequency response of the thermophysical system at the pump frequency. Finally, we can feed this into the lock-in detector, which will isolate only the reference frequency, $\omega_{o}$.

$$
S_{\text {lock }}=D S_{\text {coll }}\left(\omega_{o}\right)
$$

where $S_{l o c k}$ is the signal from the lock-in that we will use for analysis, which can be explicitly determined depending on the other frequency components of the pump and probe and $D$ is a constant 
associated with gain from the electronics.

For a continuous wave pump and probe in FDTR

$$
\begin{gathered}
\mathbf{g}(\omega)=\mathbf{g}_{o} \delta\left(\omega-\omega_{o}\right) \\
\mathbf{p}(\omega)=\mathbf{p}_{o} \\
S_{l o c k}=\mathfrak{C}_{c w} \mathfrak{B}\left(\omega_{o}\right) \\
\mathfrak{C}_{c w}=2 \pi \beta D \alpha E_{0} E_{1}
\end{gathered}
$$

This is the simplest of solutions where the signal is dictated only by the modulation frequency and the signal is proportional to the energy in the pump, $E_{0}$ and probe $E_{1}$ multiplied by the absorptivity, $\alpha$. For a pulsed pump and continuous wave probe in FDTR

$$
\begin{gathered}
\mathbf{g}(\omega)=\mathbf{g}_{o} \sum_{n=1}^{\infty} \delta\left(\omega-\omega_{o}-n \omega_{s}\right) \\
\mathbf{p}(\omega)=\mathbf{p}_{o} \\
S_{\text {coll }}(\omega)=\mathfrak{C}_{c p}^{\prime} \sum_{n=1}^{\infty} \mathfrak{B}\left(\omega_{o}-n \omega_{s}\right) \delta\left(\omega-\omega_{o}-n \omega_{s}\right) \\
S_{l o c k}=\mathfrak{C}_{c p} \mathfrak{B}\left(\omega_{o}\right) \\
\mathfrak{C}_{c p}=\beta D \alpha E_{0} E_{1} \omega_{s}
\end{gathered}
$$

where $\omega_{s}$ is the pulse frequency. Here you can see that the information that the photodetector collects is much more substantial than what the lock-in detector isolates. The lock-in detector will reject all of the $n$ values that do not correspond to the reference frequency, so you still resolve the known FDTR signal. This mathematical result, however, demonstrates that the information with respect to the pulses is carried by the CW probe and, as mentioned previously, one could use alternative detection electronics to reconstruct this information.

Finally, in the case of the pulsed pump and pulsed probe, we must take into account the temporal offset between the two pulses. This is the situation for TDTR. This treatment is more mathematically 
extensive than the final lock-in signal for FDTR, so I will not delve into the derivation of this component since it is well covered in a number of very well known manuscripts [24-26].

$$
\begin{gathered}
\mathbf{g}(\omega)=\mathbf{g}_{o} \sum_{n=1}^{\infty} \delta\left(\omega-\omega_{o}-n \omega_{s}\right) \\
\mathbf{p}(\omega)=\mathbf{p}_{o} e^{-i \omega \tau} \sum_{m=1}^{\infty} \delta\left(\omega-m \omega_{s}\right) \\
S_{\text {coll }}(\omega)=\mathfrak{C}_{p}^{\prime} \sum_{n=1}^{\infty} \sum_{m=1}^{\infty} \mathfrak{B}\left(\omega_{o}-m \omega_{s}\right) \delta\left(\omega-\omega_{o}-(n+m) \omega_{s}\right) e^{-i m \omega_{s} \tau} \\
S_{l o c k}=\mathfrak{C}_{p} \sum_{n=1}^{\infty} \mathfrak{B}\left(\omega_{o}-n \omega_{s}\right) e^{-i n \omega_{s} \tau} \\
\mathfrak{C}_{p}=\frac{\beta D \alpha E_{0} E_{1} \omega_{s}^{2}}{2 \pi}
\end{gathered}
$$

where $\tau$ is the pump-probe delay time. The concise results of these functional forms are summarized in Table 2.

Table 2: Summary of Lock-In Signals for Thermoreflectance Measurements Using Continuous Wave or Pulsed Pump Probe Configurations

\begin{tabular}{c|c||c|c|} 
& & & \\
Pump Type & Probe Type & Lock-in Signal & Prefactor \\
\hline CW & CW & $\mathfrak{B}\left(\omega_{0}\right)$ & $\beta D \alpha E_{0} E_{1}$ \\
Pulse & CW & $\mathfrak{B}\left(\omega_{0}\right)^{\dagger}$ & $\beta D E_{0} E_{1} \omega_{s}$ \\
Pulse & Pulse & $\sum_{n=1}^{\infty} \mathfrak{B}\left(\omega_{o}-n \omega_{s}\right) e^{-i n \omega_{s} \tau}$ & $\beta D \alpha E_{0} E_{1} \omega_{s}^{2} / 2 \pi$
\end{tabular}

$\dagger$ While the lock-in signal does not capture the pulsed response of the pump, the information is collected by the photodiode and can theoretically be extracted using other electronics.

With this formulation, we now have all of the pieces needed to relate the signal output by the lock-in amplifier, $S_{l o c k}$, to the thermophysical parameters contained in the function $\mathfrak{B}$. Specifically, the previously referred to $X$ and $Y$ signals are the real and imaginary parts of $S_{l o c k}$, respectively. This means that at every pump-probe delay time and/or reference frequency, given a certain lock-in signal, the thermo-physical parameters of the multilayer system can be determined. 


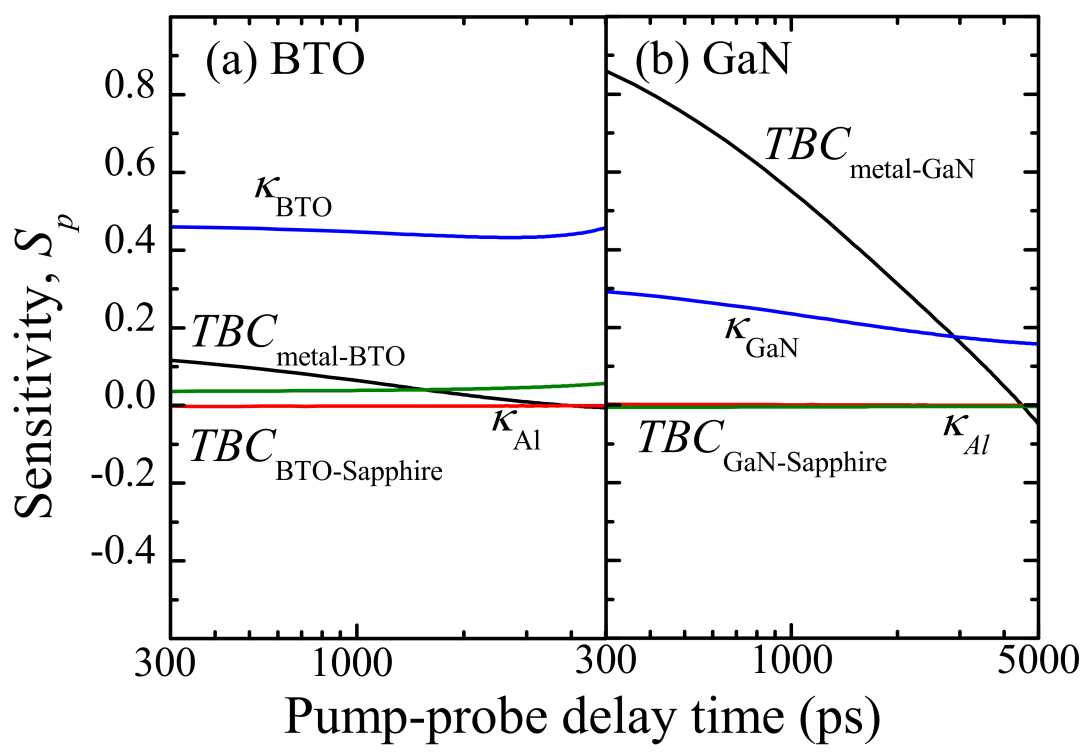

Figure 3.8: Sensitivity, S, as a function of time of our TDTR measurements and analysis to various thermophysical parameters, $p$, in our samples. We plot the thermal sensitivities to the thermal boundary conductances and the thermal conductivities of layers of interest in published (a) GaN and (b) $\mathrm{BaTiO}_{3}$ measurements [95, 263].

In terms of dealing with the constant, $\mathfrak{C}_{x}$, for each of these signals, this is generally handled by either using a component that will involve the ratio of the in-phase and out-of-phase components (which will cancel out the constants) or to normalize the signal and compare a normalized model.

\subsection{TDTR Sensitivity Analysis}

A major consideration when using TDTR to measure thermal properties is the sensitivity of the measurement to a property of interest. In order to determine this sensitivity, we employ a method outlined by Costescu et al. which relates changes in a given property to changes in the modeled decay of the thermoreflectance signal through a logarithmic derivative [264]. Mathematically the sensitivity factor $S_{p}$ is determined as follows

$$
S_{p}=\frac{\delta \ln \mathcal{S}}{\delta \ln p}
$$

where $p$ is a thermo-physical parameter of interest (such as the thermal conductivity or thermal boundary conductance of a layer) and $\mathcal{S}$ is a fitted component of the thermoreflectance decay between pulses (such as the in-phase or out-of-phase signal of the lock-in amplifier, or the ratio of the two). An example of this metric is shown in Fig. 4.4 where we compare the sensitivities to the thermal boundary conductances and the thermal conductivities of two different material systems when fitting 
the ratio of the in-phase and out-of-phase TDTR signals $[95,263]$. Intuitively, the magnitude of the sensitivity parameter indicates how confidently we can measure that parameter. Another aspect of this analysis is the difference in curvature of any two parameters. A general rule of thumb is that distinctly different curvatures allows fitting for those parameters simultaneously, which is the case for measuring the thermal boundary conductance across a metal/GaN interface and thermal conductivity of GaN, as shown in Fig. 4.4b.

The major difference between Fig. 4.4a and Fig. 4.4b demonstrates the dominance of large thermal resistors in a TDTR measurement. One dimensional thermal diffusion through multiple layers and interfaces can be roughly conceptualized by a thermal series resistor network, and TDTR is most sensitive to the largest thermal resistor in that network. This means for a layer with very low comparative thermal conductivity, such as a thin film of $\mathrm{BaTiO}_{3}$ between aluminum and sapphire, the TDTR measurement will be most sensitive to the thermal conductivity in that layer. This is an important consideration to take into account when planning the measurement of a thermophysical parameter in a multilayer stack. In the case of Fig. 4.4a a measurement of the thermal boundary conductance between the $\mathrm{BaTiO}_{3}$ and the metal transducer would be very difficult to make accurately without confidence in the value of the thermal conductivity, since the sensitivity to the thermal conductivity is high and the sensitivity to the thermal boundary conductance is low.

Opportunity for Further Investigation: The sensitivity to the thermal boundary conductance adjacent to low thermal conductivity materials is generally low. For a boundary in series with a low thermal conductivity layer, the decay of the thermoreflectance will be dominated by the thermal conductivity of the layer and small changes in the thermal boundary conductance will result in small changes in the overall signal characteristics. This issue is often overlooked in measurements of thermal boundary conductance adjacent to thermally resistive materials such as solid-liquid thermal boundary conductance. There is an opportunity here to address this issue comprehensively and also use in depth analysis of measurement sensitivity to identify systems that can accurately be measured using multi-layer thermal diffusion techniques such as TDTR. Precise measurement can only be achieved in systems with very low thermal boundary conductances, in the case of liquids this would be a solid-liquid interface with a low work of adhesion such as a hydrophobic solid and water or a an oleophobic solid and oil. 


\section{Phonon Interactions with Grain Boundaries in $\mathrm{BaTiO}_{3}$}

In this chapter I investigate the interaction of phonons with grain boundaries in thin films of barium titanate $\left(\mathrm{BaTiO}_{3}\right) \cdot \mathrm{BaTiO}_{3}$ is a material that is a primary constituent in multilayer ceramic capacitors and other electronic components that necessitate size scaling for technological advancement. Spectrally, we find that the grain boundaries scatter long wavelength phonons, demonstrating the spectral nature of phonons in oxides (a characteristic that has been refuted in literature focused on complex oxides). Much of the work in this chapter is taken from Donovan et al. "Spectral phonon scattering effects on the thermal conductivity of nano-grained barium titanate," which was published Applied Physics Letters in 2014 [95].

Barium titanate $\left(\mathrm{BaTiO}_{3}\right)$ is a widely used material in the electronics and ceramics industry due to its favorable and well known dielectric and ferroelectric properties [5, 32, 45, 265]. For example, $\mathrm{BaTiO}_{3}$ is the primary constituent in multilayer ceramic capacitors [266], a multi-billion dollar industry that necessitates size scaling of their components into the nanometer range for continued growth [8]. While the dielectric and structural properties of $\mathrm{BaTiO}_{3}$ have been widely studied, less is known about the thermal properties. Relatively large discrepancies in the reputed values of the thermal conductivity of $\mathrm{BaTiO}_{3}$ exist in literature; particularly, sub-micron-grained samples show wide variation in the reported thermal conductivity. Having a robust understanding of the effects of nano-scale features on thermal properties is of paramount importance as devices and device packaging continues to scale. $\mathrm{BaTiO}_{3}$ is not only a material system that is technologically relevant with uncertain thermal properties, it is also a system that allows for the exploration of the effects of grain boundaries on phonon transport. With the processing techniques described below, it is possible to extract clear dependancies of grain boundary effects on thermal conductivity.

In a previous work [94], Foley et al. has studied the role of phonon scattering mechanisms on the room temperature thermal conductivity in nano-grained $\mathrm{SrTiO}_{3}$. In this simple perovskite system with a cubic crystal structure, grain sizes as large as $100 \mathrm{~nm}$ can reduce the thermal conductivity of thin film $\mathrm{SrTiO}_{3}$, alluding to the relative length scales of heat carrying mean free paths in this complex oxide. This result suggested that a gray approximation to phonon transport may not be valid in 
ferroelectric oxides $[69,111,132]$. This begs the question whether a spectrum of mean free paths will contribute to the thermal conductivity in ferroelectric materials with more complex crystal structures and complicated atomic arrangements. The phonon transport processes in $\mathrm{BaTiO}_{3}$ with varying grain sizes across a range of temperatures give insight into the fundamental thermal scattering mechanisms and length scales dictating thermal conductivity in $\mathrm{BaTiO}_{3}$ and other ferroelectric perovskites.

To understand phonon scattering mechanisms and their effects on thermal conductivity in $\mathrm{BaTiO}_{3}$, I have studied the influence of grain size on thermal conductivity in thin films over temperatures ranging from 200 to 500 K. In a collaboration with Jon Ihefeld at Sandia National Laboratories, we synthesize a series of nanograined (ng) $\mathrm{BaTiO}_{3}$ thin films via chemical solution deposition [267] and measure their thermal conductivities with TDTR [24-26]. I show a consistent trend in the thermal conductivity of the $\mathrm{BaTiO}_{3}$ films as a function of grain size, similar to what Foley et al. [94] has previously observed in nanograined $\mathrm{SrTiO}_{3}$ films; this consistency in the effects of nano-structuring on the thermal conductivity of $\mathrm{BaTiO}_{3}$ does not appear among previous works [268, 269]. My data agree well with predictions for the grain boundary scattering effects on the thermal conductivity of $\mathrm{BaTiO}_{3}$ using the simplified semi-classical model discussed later in this chapter. The temperature trends suggest that phonon-boundary scattering events, both at grains and the film/substrate boundary, are the dominant source of thermal resistance. This is in line with my model, which assumes a spectrally dependent phonon mean free path in the $\mathrm{BaTiO}_{3}$ system. The influence of 36-63 nm diameter grains on the thermal conductivity of $\mathrm{BaTiO}_{3}$ indicate phonons with this characteristic length scale can contribute to the thermal conductivity of single crystal $\mathrm{BaTiO}_{3}$, which is contrary to the typical kinetic theory calculation of the $\sim 2 \mathrm{~nm}$ mean free path assuming a gray medium. Therefore, this work demonstrates the spectral nature of the thermal mean free paths of phonons in $\mathrm{BaTiO}_{3}$ with typical length scales well beyond that predicted by a gray approximation using kinetic theory [270].

\subsection{Literature Thermal Conductivity of $\mathrm{BaTiO}_{3}$}

In contrast to the variety of conflicting values in current works in literature, this work elucidates the effects of grain size on thermal conductivity of thin films of $\mathrm{BaTiO}_{3}$ using a robust measurement technique, not susceptible to radiative loss, and a consistent sample set with uniform film thickness and well characterized materials. Current literature values [269] regarding the effects of nano-structuring 
on $\mathrm{BaTiO}_{3}$ range from studies with $150 \mathrm{~nm}$ grains exhibiting near-bulk thermal conductivities of 5.1 $\mathrm{W} \mathrm{m} \mathrm{m}^{-1} \mathrm{~K}^{-1}$ to studies with grain sizes of $100 \mathrm{~nm}$ exhibiting thermal conductivities of $10.22 \mathrm{~W} \mathrm{~m}^{-1}$ $\mathrm{K}^{-1}$, which is over twice that reported for the bulk thermal conductivity of $\mathrm{BaTiO}_{3}[71]$.

In terms of the thermal conductivity of polycrystalline/nano-grained $\mathrm{BaTiO}_{3}$, there exists no clear agreement among the reported values in the literature. Jezowski et al. [268] measured the thermal conductivity of polycrystalline $\mathrm{BaTiO}_{3}$. The values reported for a ceramic with $100 \mathrm{~nm}$ grains were nearly twice the thermal conductivity of bulk single crystal values reported elsewhere. This is also in contrast to values reported by Davitadze et al. [269] on $1 \mu \mathrm{m}$ thin films with roughly $150 \mathrm{~nm}$ grains. Jezowski et al. [268] also found that for the ceramics with $30 \mathrm{~nm}$ grains the thermal conductivity was around $1.7 \mathrm{Wm}^{-1} \mathrm{~K}^{-1}$, which is lower than bulk and to be expected with nano-structuring. They explain the wide variation in their data from potential radiation losses and photon thermal conductivity from the surface of their samples due to an optically black ceramic, a characteristic that could also indicate a highly defective material that could result in an increased concentration of electronic carriers as well. Therefore, the measured values reported for their ceramics do not represent the intrinsic phonon thermal conductivity of nano-grained $\mathrm{BaTiO}_{3}$.

Particularly in nano-grained and thin film systems, there have been a number of conflicting values reported in the literature for the thermal conductivity of barium titanate $\left(\mathrm{BaTiO}_{3}\right)$; these are summarized in Table 3. In the mid 1900's, bulk single crystal thermal properties were determined by Mante and Volger using steady state heat flow techniques [71]. More recent measurements of the thermal conductivity of bulk, single crystalline $\mathrm{BaTiO}_{3}$ have reported values slightly lower than the original measurements [69]. In 2004, the thermal conductivity of bulk, ceramic $\mathrm{BaTiO}_{3}$ was shown to have slightly lower values than the single-crystalline material, most likely due to point defects or grain boundaries introduced by the sintering process [271].

The wide variety in values for thermal conductivity illustrates the nuances involved with thermal measurements of ceramics, along with the need for consistent, accepted values of the thermal conductivity of nano-grained $\mathrm{BaTiO}_{3}$. Issues that arise from the processing, measurement, and analysis of these properties such as porosity, defectivity, grain size, impurity contamination, and film thickness can significantly alter findings if the system in question is not fully characterized and understood. The study that I present in this dissertation establishes a consistent set of data for measured thermal conductivity of nanograined and single crystalline $\mathrm{BaTiO}_{3}$. 
Table 3: Various Literature Values of Thermal Conductivity $(\kappa)$ for Bulk and Nano-structured $\mathrm{BaTiO}_{3}$ at $\mathrm{Room}$ Temperature

\begin{tabular}{c|c|c|c} 
Data Source (year) & $\begin{array}{c}\text { Avg. } \kappa \text { of } \mathrm{BaTiO}_{3} \\
\text { @ RT }\left(\mathrm{Wm}^{-1} \mathrm{~K}^{-1}\right)\end{array}$ & Sample Type & Measurement Method \\
\hline \hline Mante \& Volger Physica (1967) & 5.7 & Bulk Single Crystal & Steady State Heat Flow \\
Tachibana et al. APL (2008) & 3.4 & Bulk Single Crystal & Bulk Meas System \\
He et al. Therm Acta (2004) & 2.7 & Bulk Ceramic & $3 \omega$ method \\
\hline Davitadze et al. APL (2002) & 5.1 & $1 \mu \mathrm{m}$ Thin Film w $150 \mathrm{~nm}$ Grains & Axial Stationary Flow \\
Jezowski et al. APL (2007) & 10.22 & Bulk Ceramic w/ 100nm Grains & Axial Stationary Flow \\
Jezowski et al. APL (2007) & 1.73 & Bulk Ceramic w/ 30nm Grains & TDTR \\
& $2.17 \pm 0.22$ & $175 \mathrm{~nm}$ Epitaxial Thin Film & TDTR \\
This Work & $1.71 \pm 0.06$ & $175 \mathrm{~nm}$ Thin Film w/ $63 \pm 2.7 \mathrm{~nm}$ Grain & TDTR \\
& $1.42 \pm 0.07$ & $175 \mathrm{nmThin}$ Film w/ $52 \pm 5.1 \mathrm{~nm}$ Grain & TDTR \\
& $1.27 \pm 0.1$ & $175 \mathrm{~nm}$ Thin Film w/ $47 \pm 7.4 \mathrm{~nm}$ Grain & TDTR \\
& $1.08 \pm 0.07$ & $175 \mathrm{~nm}$ Thin Film w/ $42 \pm 5.0 \mathrm{~nm}$ Grain & TDTR \\
\hline
\end{tabular}

Utilizing the sensitivity of TDTR and well understood processing with full characterization of our materials, I show the effects of grain boundaries on the thermal conductivity of $\mathrm{BaTiO}_{3}$ and illustrate the typical length scales of the thermal phonon mean free paths in the system. Taken with previous work on $\mathrm{SrTiO}_{3}$ [94], this body of work suggests the spectral nature of phonons in complex oxide perovskites in general.

\section{2 $\quad \mathrm{BaTiO}_{3}$ Film Fabrication and Characterization}

Fabrication and characterization of these films were conducted by Dr. Jon Ihlefeld at Sandia National Labs. $\mathrm{BaTiO}_{3}$ thin films were prepared via chemical solution deposition utilizing a varying final anneal temperature to modify the final grain size [145]. A chelate chemistry comprised of barium acetate, titanium isopropoxide, acetylacetonate, propionic acid, and methanol was used and is described in more detail in prior work [272]. Cation precursors were assayed prior to batching to ensure a stoichiometric composition. A single film was spin cast onto a polished polycrystalline alumina substrate (CoorsTek Superstrate), which was chosen to minimize potential texturing effects that may occur due to local epitaxy with a sapphire substrate. Films nine layers in total thickness (ca. $\sim 175 \mathrm{~nm}$ as-fired thickness) were prepared with an $800^{\circ} \mathrm{C}$ anneal after every layer to assure that all carbonaceous species were removed and that a dense film was prepared [267, 273]. The substrate was then diced and each section annealed at temperatures between 850 and $1000^{\circ} \mathrm{C}$ in $50^{\circ} \mathrm{C}$ increments for one hour in air to modify the grain size.

A $175 \mathrm{~nm}$ thick epitaxial $\mathrm{BaTiO}_{3}$ film was grown on a (001)-oriented $\mathrm{SrTiO}_{3}$ single crystalline 
substrate via $30^{\circ}$ off-axis $\mathrm{RF}$ magnetron sputtering from a $75 \mathrm{~mm}$ diameter sintered $\mathrm{BaTiO}_{3}$ target. The $\mathrm{SrTiO}_{3}$ surface was prepared with a $\mathrm{TiO}_{2}$ termination prior to growth [274]. The film was grown with a substrate temperature of $700^{\circ} \mathrm{C}$ in a 20 mTorr, 5:1 ratio argon:oxygen atmosphere with a growth rate of $2.7 \mathrm{~nm} / \mathrm{min}$. The film was allowed to cool in the growth atmosphere to minimize the formation of oxygen point defects.

Film phase assemblage and orientation was assessed with X-ray diffraction using a Philips X'Pert MPD instrument with copper $\mathrm{K} \alpha$ radiation. Figure 4.1a shows representative X-ray diffraction patterns for each film. In each case a phase pure film is identified. The solution deposited films possess no preferred orientation and therefore can be consider randomly oriented and polycrystalline. The epitaxial film possesses just peaks consistent with $c$-axis oriented $\mathrm{BaTiO}_{3}$. No evidence of other $a$-axis or reflections from other planes could be observed. Rocking curves, seen in Fig. 4.1b, measured for the substrate and film 002 peaks possessed full width at half maximum values of $0.092^{\circ}$ and $1.142^{\circ}$, respectively. The increased width of the film peak can be attributed to strain fields around misfit dislocations near the film/substrate interface [275]. 


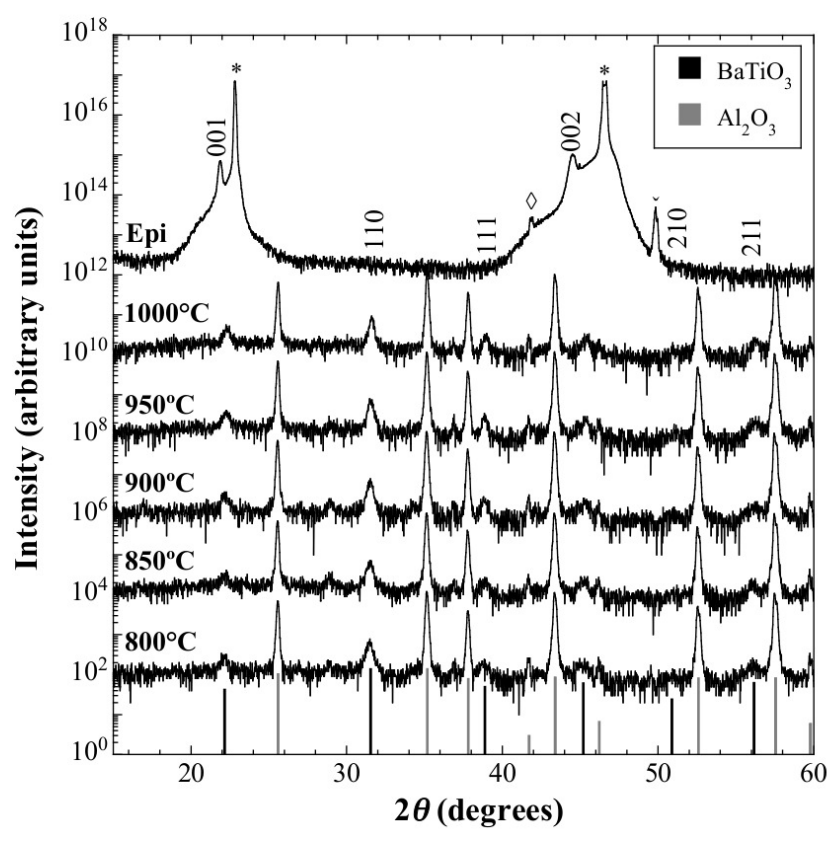

(a) XRD patterns of $\mathrm{BaTiO}_{3}$ films

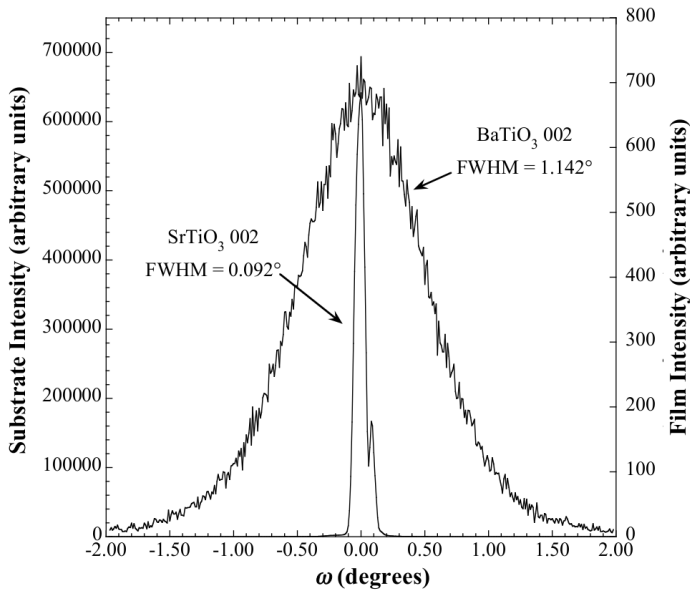

(b) XRD rocking curve $\left(\mathrm{BaTiO}_{3}\right.$ film on $\left.\mathrm{SrTiO}_{3}\right)$

Figure 4.1: (a) X-ray diffraction patterns for $\mathrm{BaTiO}_{3}$ films processed between 800 and $1000^{\circ} \mathrm{C}$ on polycrystalline alumina substrates and an epitaxial film prepared on a 001-oriented $\mathrm{SrTiO}_{3}$ substrate. The peak positions for cubic $\mathrm{BaTiO}_{3}$ and corundum structured $\mathrm{Al}_{2} \mathrm{O}_{3}$ are indicated by the bar markers [276, 277]. * denotes $\mathrm{SrTiO}_{3}$ reflections, $\diamond$ denotes a reflection of copper $\mathrm{K} \beta$ radiation, and 'is an unknown peak that we have previously observed for these $\mathrm{SrTiO}_{3}$ substrates and this diffractometer and is not believed to be a secondary phase in the film. (b) X-ray diffraction rocking curves of the substrate and film 002 reflections. Full width at half maximum (FWHM) values for the substrate and film are $0.092^{\circ}$ and $1.142^{\circ}$, respectively.

Film microstructure was assessed with scanning electron microscopy (Zeiss Supra 55VP in in-lens imaging mode and FEI Varios) and atomic force microscopy (Park Scientific Autoprobe CP). Figure 4.2 shows scanning electron micrographs of the polycrystalline films after annealing at the different temperatures. A clear increase in grain size with increasing annealing temperature is observed. Cross-sectional images reveal relatively dense microstructures (>97\% based upon image analysis, ImageJ, National Institutes of Health) for each condition and smooth interfaces with the substrate. Average grain sizes were assessed from the atomic force microscope images, seen in Fig. 4.3, using the linear intercept method [278]. Error bars on the grain size measurements represent $95 \%$ confidence intervals. 

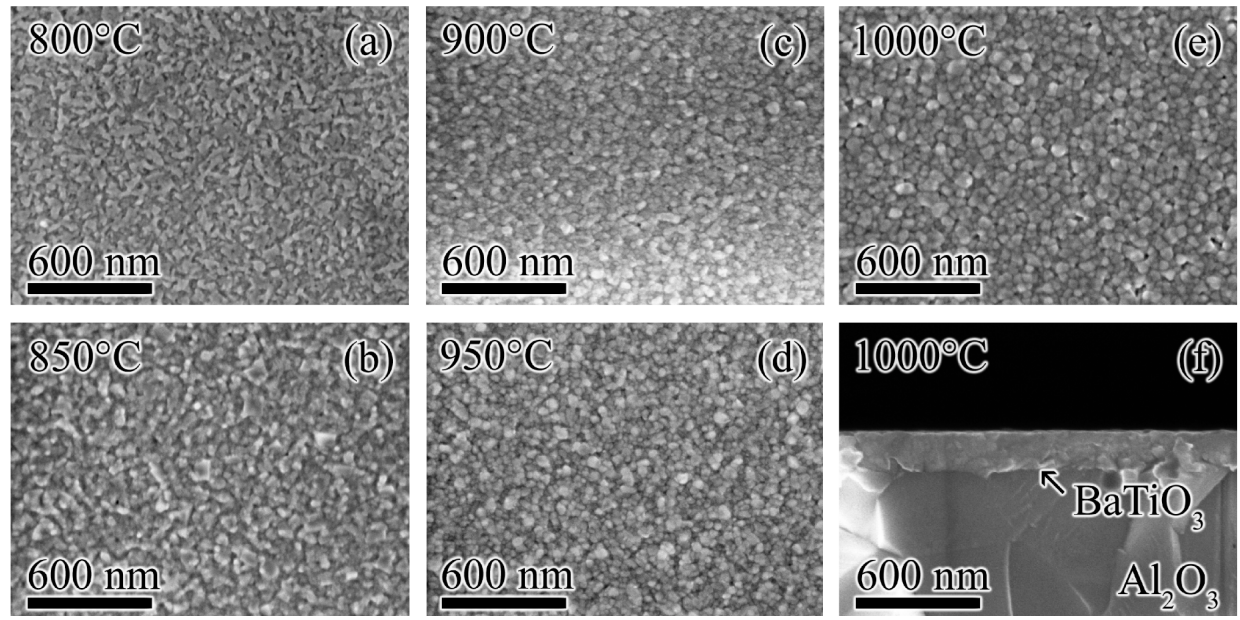

Figure 4.2: Plan-view scanning electron micrographs of polycrystalline $\mathrm{BaTiO}_{3}$ films annealed between (a) $800^{\circ} \mathrm{C}$ and (e) $1000^{\circ} \mathrm{C}$. (f) is a cross-sectional image of the $1000^{\circ} \mathrm{C}$ annealed $\mathrm{BaTiO}_{3}$ film.
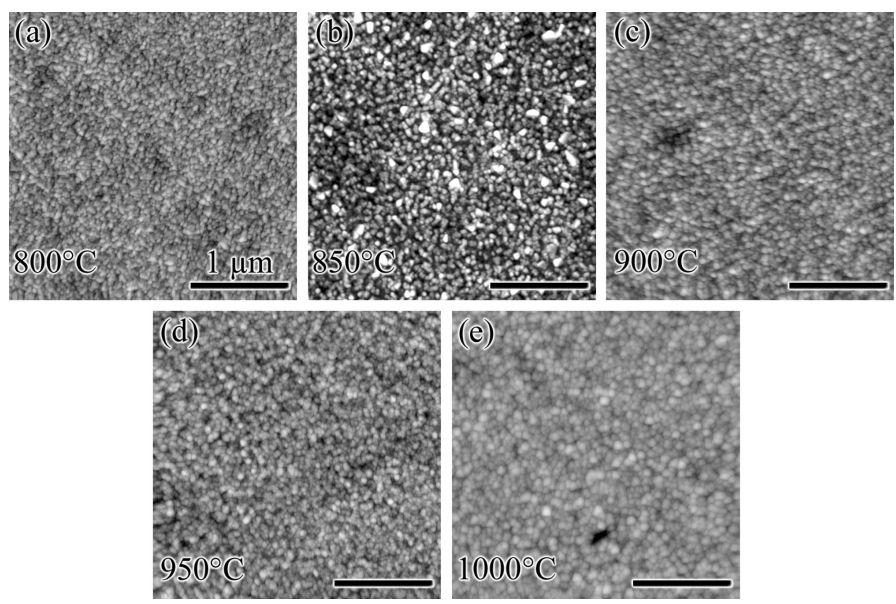

Figure 4.3: $3 \mu \mathrm{m} \times 3 \mu \mathrm{m}$ contact mode topographic atomic force microscope images of polycrystalline $\mathrm{BaTiO}_{3}$ films annealed at (a) $800^{\circ} \mathrm{C}\left(36 \pm 4.9 \mathrm{~nm}\right.$ grain), (b) $850^{\circ} \mathrm{C}\left(42 \pm 5.0 \mathrm{~nm}\right.$ grain), (c) $900^{\circ} \mathrm{C}\left(47 \pm 7.4 \mathrm{~nm}\right.$ grain), (d) $950^{\circ} \mathrm{C}$ $\left(52 \pm 5.1 \mathrm{~nm}\right.$ grain), and (e) $1000^{\circ} \mathrm{C}(63 \pm 2.7 \mathrm{~nm}$ grain $)$.

\subsection{Thermal Measurements and Phonon-Grain Boundary Interactions}

I measured the thermal conductivity of the nanograined films with time domain thermoreflectance [24-26]. The thermal conductivities of the nano-grained thin films were greatly reduced compared to bulk values. Furthermore, the average size of the grains in each sample significantly affected its thermal conductivity. A clear reduction in the thermal conductivity of the ng- $\mathrm{BaTiO}_{3}$ compared to the epitaxial film is observed even for grain sizes as large as $63 \mathrm{~nm}$. As expected, the epitaxial thin film sample shows a higher thermal conductivity than any nano-grained samples, but is a factor of two lower than the reported bulk values $[69,71]$, which is attributed mainly to film size effects. 
This coincides well with data taken by Foley et al. [94] on $\mathrm{SrTiO}_{3}$ showing a similar dependance of thermal conductivity with grain size and a similar reduction from bulk values of approximately 11 $\mathrm{W} \mathrm{m}^{-1} \mathrm{~K}^{-1}$.

In the analysis of the TDTR data, I assume literature values for the heat capacity of Al [279], $\mathrm{BaTiO}_{3}$ [280], and $\mathrm{Al}_{2} \mathrm{O}_{3}$ [281], as well as the thermal conductivity of $\mathrm{Al}_{2} \mathrm{O}_{3}$ [282]. I confirmed a more accurate thickness of $79 \pm 2 \mathrm{~nm}$ for the Al layer location with picosecond acoustics [246, 247]. I assume a reduced thermal conductivity of the $\mathrm{Al}$ film based on electrical resistivity measurements and the Weidemann-Franz law, though we are insensitive to this parameter in the TDTR analysis. In this analysis, I also treat the thermal boundary conductance for the $\mathrm{Al} / \mathrm{BaTiO}_{3}$ interface as a free parameter in our model fit, our system is relatively insensitive to this as well as the backside interface conductance. Reported error in the thermal conductivity measurement arrises from a combination of small variation of thicknesses of constituent materials, particularly in the Al transducer, and measurement of a number of different sites on the surface of each sample. Using laser spot sizes of $62.9 \mu \mathrm{m}$ and $13.2 \mu \mathrm{m}$ diameters for the pump and probe, respectively, allows a valid assumption of a one dimensional heating event [26]. Room temperature measurements, along with an analytical model for the grain size limited thermal conductivity are shown in Fig. 4.4. Additional measurements over temperature were performed through a transparent window in a cryostat cooled by liquid nitrogen, these are featured later in the chapter in Fig. 4.5.

The model of spectral phonon transport and grain boundary scattering in our $\mathrm{BaTiO}_{3}$ films is based on semi-classical formalisms of the kinetic theory expression for thermal conductivity discussed in Sect. 2.2. In this study, I approximate the phonon dispersion by a sine-type relation and only take into account contributions to the thermal conductivity from the acoustic branches of the phonon dispersion. The boundary scattering is taken into account in the total scattering time by Matthiessen's rule given by

$$
\frac{1}{\tau}=A T \omega^{2} \exp \frac{-B}{T}+D \omega^{4}+\frac{v}{d_{f i l m}}+\frac{v}{d_{g b}}
$$

In this expression, from left to right, we account for the phonon-phonon scattering, impurity scattering, film thickness scattering, and grain boundary scattering respectively. Here, $T$ is the sample temperature, $\omega$ is the phonon frequency, $d$ is the appropriate thickness or grain size, and $A, B$, and 


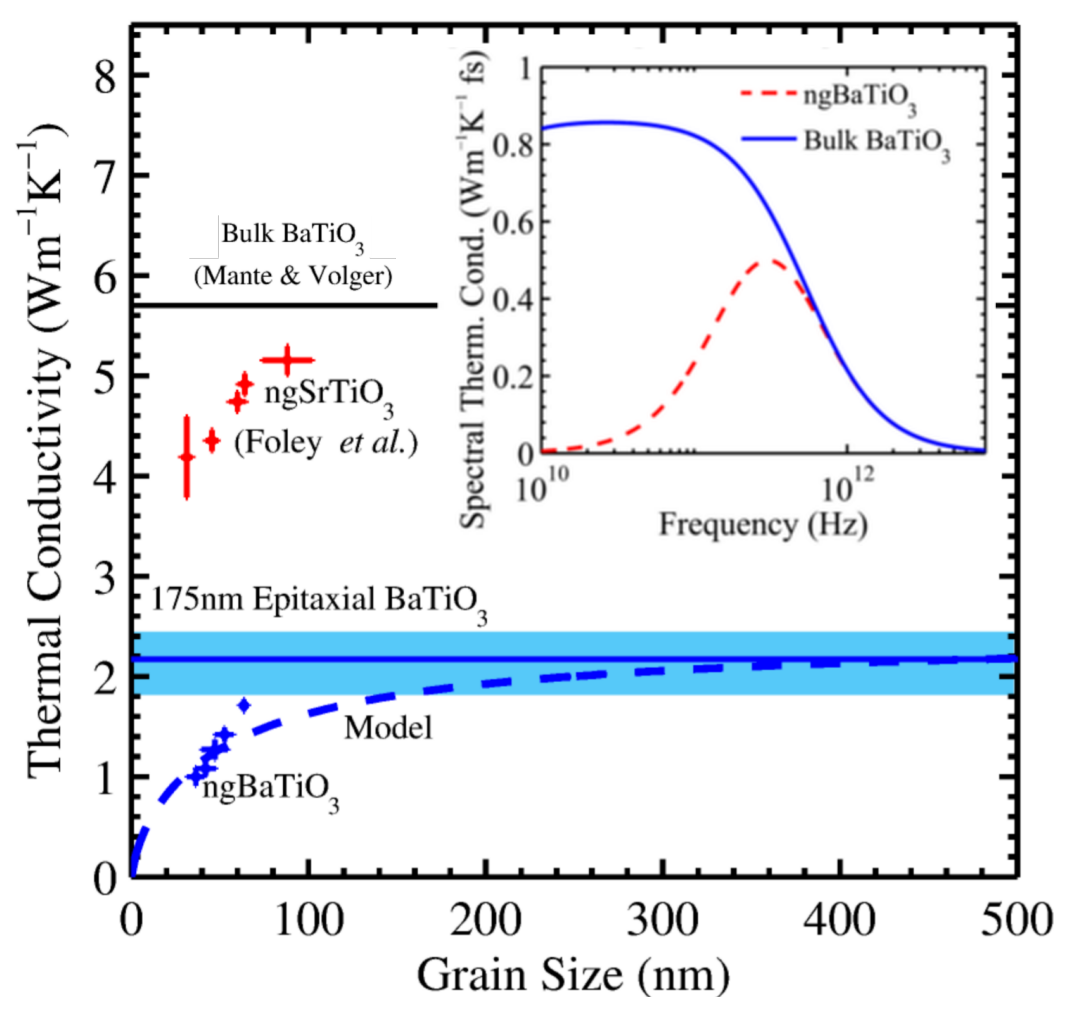

Figure 4.4: Room temperature thermal conductivity of $\mathrm{BaTiO}_{3}$ as a function of grain size along with the measured thermal conductivity of a single crystal film. For comparison, we also show the thermal conductivity of bulk, single crystal $\mathrm{BaTiO}_{3}$ reported by Mante and Volger [71]. The dashed line represents our model calculations for $\mathrm{BaTiO}_{3}$ as a function of grain size. Also included is similar data for another common perovskite, $\mathrm{SrTiO}_{3}$, from Foley et al. [94].

$D$ are fitting parameters associated with the bulk scattering processes. These fitting parameters are determined initially by fitting the model to bulk, single crystal data [71] without the additional grain boundary and interface scattering components and are given as $A=700 \times 10^{-17} \mathrm{~s} \mathrm{~K}^{-1}, B=165 \mathrm{~K}$, and $D=1 \times 10^{-35} \mathrm{~s}^{3}$.

The simple sine-type relation to model the phonon dispersion in this system [21] has been shown to be a good prediction for mean free path and thermal conductivity elsewhere in literature [283, 284]. This dispersion model yields a frequency dependance of

$$
\omega(k)=\omega_{c} \sin \left[\frac{k a}{2}\right]
$$

where $\omega$ is the phonon frequency, $\omega_{c}$ is the cutoff frequency, $k$ is the phonon wavevector, and $a$ is the lattice constant. I model the group velocity, $v_{g}$ correspondingly by 


$$
v_{g}=v_{o} \sqrt{1-\left(\frac{\omega}{\omega_{c}}\right)^{2}}
$$

where $v_{o}$ is the sound speed for a given phonon polarization in the material. This leads to a modeled density of states in the form of

$$
D(\omega)=\frac{2\left(\frac{2}{a} \sin ^{-1}\left(\frac{\omega}{\omega_{c}}\right)\right)^{2}}{\pi \omega_{c} \sqrt{1-\left(\frac{\omega}{\omega_{c}}\right)^{2}}}
$$

Using this density of states, phonon velocity, scattering time, and a Bose-Einstein distribution, I am able to accurately model the thermal conductivity of $\mathrm{BaTiO}_{3}$ and recreate temperature trends in the bulk data using the fitting parameters given above.

For comparison to the spectral model described above, I also include a gray model [270] in which there is a single mean free path of the phonons in a system at any given temperature. To determine the values of this gray mean free path, I turn to literature values for heat capacity and thermal conductivity and rearrange the equation

$$
\kappa=\frac{1}{3} C \nu \lambda
$$

to

$$
\lambda_{\text {gray }}(T)=\frac{\kappa(T)}{\frac{1}{3} C(T) \nu}
$$

This enables the use of temperature dependent literature values for thermal conductivity, $\kappa$, and heat capacity, $C$, along with the speed of sound, $\nu$, to determine the gray mean free path of phonons in bulk $\mathrm{BaTiO}_{3}$ as a function of temperature. By this process, at room temperature, the gray model predicts a phonon mean free path in bulk $\mathrm{BaTiO}_{3}$ of $\lambda_{b u l k}=2.3 \mathrm{~nm}$. This result in itself would suggest that introducing grain-boundaries from 36 - $63 \mathrm{~nm}$ grains should have no effect on the thermal conductivity in the system, which is very clearly not the case. To further illustrate this, and validate the spectral model of thermal conductivity in this system, I analyze the models over temperature. Using the same approach to formulate scattering time as discussed previously, I 
determine the total scattering predicted by the gray model by Matthiessen's rule.

$$
\frac{1}{\tau}=\nu\left(\frac{1}{\lambda_{\text {gray }}}+\frac{1}{d_{f i l m}}+\frac{1}{d_{g b}}\right)
$$

The resulting prediction for thermal conductivity of the thin film, nano-grained system, with 36 $\mathrm{nm}$ grains as an example, is shown in Fig. 4.5. This prediction does not accurately depict the data measured. The gray model predicts that the thin film, nano-grained $\mathrm{BaTiO}_{3}$ should show only a slight deviation from the bulk thermal conductivity at operating temperatures. The trend given by the gray model also helps to illustrate the quality of fit of the simple spectral model included in the manuscript to the data measured.

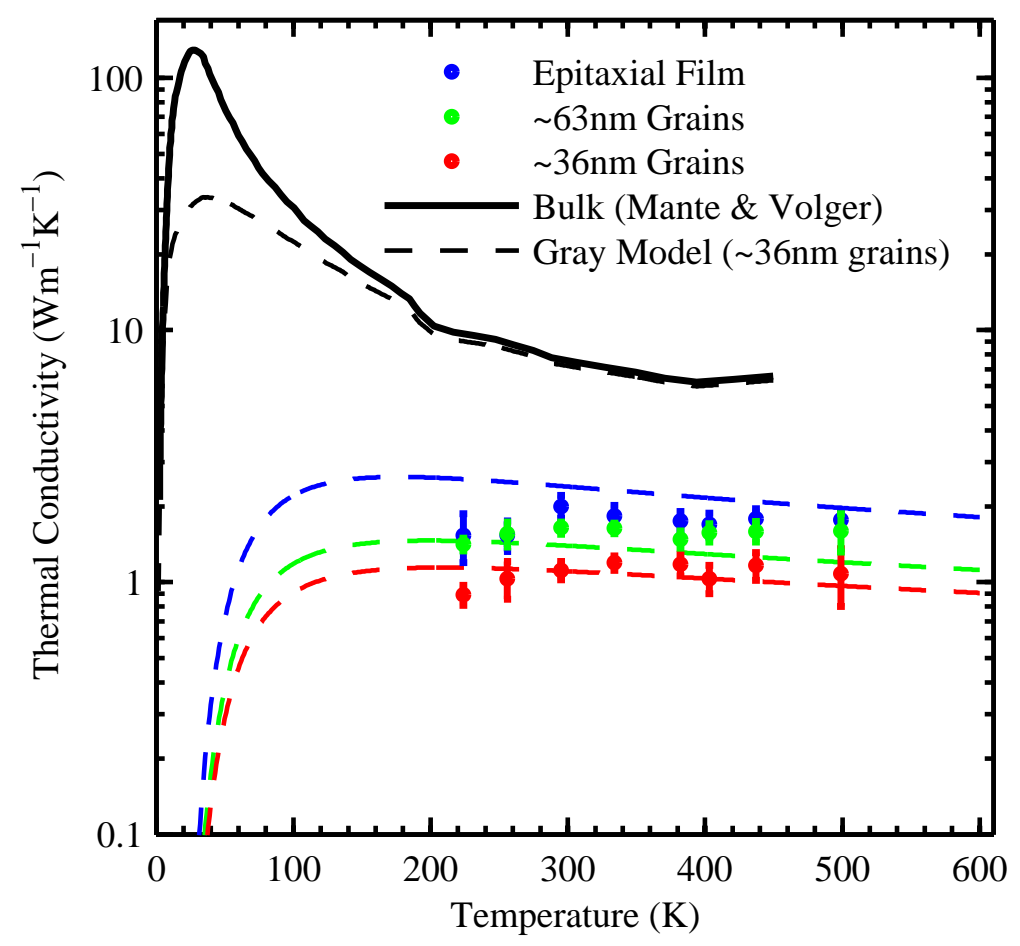

Figure 4.5: Thermal conductivity of $\mathrm{BaTiO}_{3}$ over varying sample temperature for two nano-grained and a single crystal thin film with respective modeled trends. Included is the prediction by the gray model for the $36 \mathrm{~nm}$ grain, thin film samples, showing very little deviation from bulk values [71] when neglecting the spectral nature of the phonons in the system.

The dependance of thermal conductivity on grain size shown in Fig. 4.4 illustrates limitation of the phonon mean free path spectrum by grain boundaries. With smaller grains, phonons with mean free paths greater than the grain sizes scatter more readily, translating into a reduced ability to conduct thermal energy. This is consistent with previous observations of nano-grained silicon [285] 
and our previous measurements of grain size dependence on the thermal conductivity of $\mathrm{SrTiO}_{3}$ [94]. The strong dependence of thermal conductivity on grain sizes larger than $40 \mathrm{~nm}$ implies phonons with mean free paths greater than $40 \mathrm{~nm}$ are conducting thermal energy. This directly conflicts with the picture that phonons in these complex oxides (e.g., $\mathrm{BaTiO}_{3}$ and $\mathrm{SrTiO}_{3}$ ) have non-spectral (i.e. gray) mean free paths that are $\sim 2 \mathrm{~nm}$, which has been used to analyze phonon transport in $\mathrm{SrTiO}_{3}$ previously [111]. The spectral nature of thermal conductivity in $\mathrm{BaTiO}_{3}$ is illustrated by the model used in this study, shown in the inset of Fig. 4.4. From this, it is clear that with the nano-structured sample the reduction in thermal conductivity arises from a limited contribution of the low frequency, long wavelength, phonons. At higher frequencies the contribution to thermal conductivity is identical to bulk behavior.

\section{Opportunites for Further Investigation:}

(1) A simple model for grain boundary scattering has captured the trends in the data relatively well. While this fit and theoretical form of phonon-boundary scattering leads to an accurate and efficient representation of the physical system, there is always room for improvement of models. This experiment is one where there may be an interesting investigation into the physical form of the phonon-grain boundary interaction. For instance, extension of the grain boundary scattering time to include a phonon wavelength dependence, as it is treated in some recent works in literature [286, 287], may yield some additional insight beyond the trends and confirmation of spectral scattering seen in this experiment.

(2) Thermal rectification, or the bias of heat flow in a forward direction, can be achieved by graded size effects. Using chemical solution deposition and controlled grain size, one could theoretically accomplish crossplane thermal rectification by incrementally layering $\mathrm{BaTiO}_{3}$ films of larger and larger grain size. This would establish a thermal conductivity gradient, forcing a significant difference in heat flux from the top of the film down as compared the bottom of the film up. This effect could be directly measured with TDTR using a Pt- $\mathrm{Al}_{2} \mathrm{O}_{3}$ substrate and measuring transport on opposite sides of the thermal rectifying film.

The included figures show that the spectral model I have presented fits the measured data trend well, is more appropriate than the gray model, and asymptotes towards our single crystalline measured values at large grains sizes. In this model, I assume both grain boundaries and film boundaries 
will scatter phonons equally, which accurately predicts that the epitaxial film data has a phonon mean free path limitation of the film thickness of the sample.

The thermal conductivity as a function of temperature of three samples is plotted in Fig. 4.5. Also for comparison in Fig. 4.5, I plotted the bulk data from Mante and Volger [71]. The temperature trends among the epitaxial thin film, and the two nano-grained samples (average grain sizes of 36 and $63 \mathrm{~nm}$ ) are nearly equivalent, which is the expected trend in boundary scattering limited transport and is illustrated by the included model of the system. The agreement of the spectral model of thermal conductivity and the measured data, especially when compared to a gray model, points clearly towards grain size effects directly limiting the phonon spectrum.

In bulk systems, low temperature thermal conductivity is limited by boundary and impurity scattering effects, and there is generally an increase in thermal conductivity until thermal energy increases the population of phonons in the system to the point where phonon-phonon scattering becomes dominant. This causes the characteristic hump and subsequent decrease of thermal conductivity that is inversely proportional to temperature. Upon introduction of nanoscale scattering sites, such as grain boundaries, the boundary scattering effects are so severe that the characteristic hump gets smeared out into the modeled trend shown in Fig. 4.5. At somewhat higher temperatures, the boundaries have essentially stabilized the temperature dependance of the system and hold the thermal conductivity relatively constant over that range in temperature. This trend has been well studied in silicon-based nano structures [4] and extends as well into complex oxides.

Another important variation from bulk temperature dependance of $\mathrm{BaTiO}_{3}$ seen in the nanograined samples is the behavior around phase transition temperatures. The bulk phase transition progression of $\mathrm{BaTiO}_{3}$ is well known [54], with transitions from the rhombohedral, to orthorhombic, to tetragonal, and finally to the cubic phase at $180 \mathrm{~K}, 270 \mathrm{~K}$, and $400 \mathrm{~K}$, respectively. In bulk systems, thermal conductivity and heat capacity have been shown to spike through these transition temperatures [71, 288], but in this nano-structured system I do not observe any measurable nonlinearities or inflections across these temperatures. It is well demonstrated in both bulk ceramics [6, 289, 290] and thin films [145] that as grain size is decreased below $1 \mu \mathrm{m}$, the phase transitions tend to pinch together and diminish in their amplitude. Using a metric of the dielectric constant, Frey et al. [291] were able to show a clear low temperature phase transition in $\mathrm{BaTiO}_{3}$ samples that had grain sizes on the order of $1 \mu \mathrm{m}$, yet the peak in dielectric constant for grain sizes below $100 \mathrm{~nm}$ is 
nearly completely suppressed. Accompanying this suppression of peak permittivity was a decrease in the latent heat of transition at the Curie temperature [289]. Ihlefeld et al. [145] show this same relative permittivity trend as observed in bulk ceramics for thin film samples similar to those used in this study, with nearly full peak suppression at grain sizes of about $76 \mathrm{~nm}$. While we know from previous work in similar systems [145] that these samples are still in a non-centrosymmetric crystal structured regime, since we observe no obvious influence of the orthorhombic to tetragonal or tetragonal to cubic phase transitions in both the single crystalline and nano-grained films, I hypothesize that the fine grain and thin film geometry is suppressing the influence of these phase transitions on thermal conductivity.

In summary, the nano-grained samples of barium titanate showed a significantly reduced thermal conductivity compared to bulk. The thermal conductivity trended lower with decreasing grain sizes showing a limitation of phonon mean free paths by grain boundaries. Over a range of industry and material relevant temperatures it is clear that the thermal conductivity of the samples no longer depends strongly on temperature, since the scattering component from grain boundaries dominates over any other contribution to overall phonon scattering and thermal conductivity. 


\section{$5 \quad$ Phonon Interactions with Extrinsically Controlled Point Defects in CdO}

In this chapter, I will show the tunability of the thermal conductivity of CdO using substitutional doping and control of other intrinsic defects. With the interaction between the extrinsic and intrinsic point defects, we are able to reduce total phonon scattering, increase total phonon scattering, increase electronic thermal conductivity, or decrease the total thermal conductivity simply by Dy dopant concentration. The work presented in this chapter has been published by Sachet et al. "Dysprosiumdoped cadmium oxide as a gateway material for mid-infrared plasmonics," Nature Materials (2015) [143] as well as Donovan et al. "Interplay between mass-impurity and vacancy phonon scattering effects on the thermal conductivity of doped cadmium oxide," Applied Physics Letters (2016) [144]. Featured in Donovan et al. [144], I include a model that captures the data in various doping regimes, indicating dominance of various defect interactions that occur over different dopant concentrations. This study demonstrates not only thermal tunability, but the use of thermal transport findings to further understand the complex defect interactions in oxides.

The impact of point defects on phonon dominated thermal transport has been largely addressed by experimental evidence garnered from studies of impurities in silicon and silicon-based materials [292294]. Going beyond impurity scattering in these silicon systems and looking towards oxides, previous works quantified the role of oxygen vacancy interactions with phonons in bulk materials, starting around the 1960's [78-80]. With recent advances in materials processing and characterization, a new level of capability and complexity has been introduced that is commonly being utilized in the form of thin film oxide materials. With the move to nanoscale dimensionality, the addition of phonon boundary scattering as a major concern for thermal engineering is another aspect that has been the topic of recent investigation $[82,94,95,139,147,295]$. Recent interest in thermoelectric materials as a viable source of energy scavenging along with concentration on oxides for thermal barrier coatings has encouraged more concentration on the ability to reduce thermal transport in oxides based on variability in sample geometry, nanostructure and stoichiometry. It can be even more insightful to leverage the complexity of these materials to fully tune thermal transport with the variety of controls available through advanced processing and characterization. 
Cadmium oxide is a relatively simple functional oxide material; it has a rock salt crystal structure and is used often in optical applications [296]. While the initial application pursued for these materials is in infrared plasmonics, we will see that the tuning of thermal transport via substitutional doping is quite significant and offers a wide array of opportunity in thermal engineering [143].

\subsection{CdO Synthesis and Defect Control}

CdO:Dy films were synthesized by colleagues in the Maria group at North Carolina State University via plasma assisted molecular beam epitaxy. The $\mathrm{CdO}$ samples studied in this work range in Dy doping levels from 0.0016 at.\% to 1.2 at.\% (near the solubility limit of 1.3 at.\%). Heteroepitaxial thin films were grown on $\mathrm{MgO}$ (100) substrates with a thickness of $500 \mathrm{~nm}$. Dy acts as an n-type dopant, replacing $\mathrm{Cd}$ atoms on the $\mathrm{Cd}$ site of the $\mathrm{CdO}$ crystal $\left(\mathrm{Dy}_{\mathrm{Cd}} \bullet\right.$ ). Doping with Dy encourages a reduction in oxygen vacancies in the $\mathrm{CdO}$ via Fermi-level pinning [143]. This results in a shift of the defect equilibrium to a state with a fixed substitutional impurity concentration. Considering that the difference in radius due to substitution of Dy on a Cd site is much less than that of a vacancy in an $\mathrm{O}$ site, the initial introduction of Dy into the system is accompanied with an increase in carrier mobility.

This shift in defect equilibrium is not only crucial to this work and the range of manipulation of thermal transport, but it also demonstrates the power of using functional oxide materials in thermal transport applications. In pure $\mathrm{CdO}$, there will be a baseline concentration of oxygen vacancies, the equilibrium reaction for which is given below.

$$
C d_{C d}{ }^{\times}+O_{O} \times \longleftrightarrow C d_{C d} \times+V_{O}^{\bullet \bullet}+2 n+\frac{1}{2} O_{2}(g)
$$

I have discussed the major concepts of intrinsic defect populations in functional oxides in Ch. 2.3 and will explore the impact of intrinsic defect on thermal transport in Ch. 6. In this study, using extrinsic defects, the addition of a substitutional dopant leads to promotion of oxygen atoms onto oxygen lattice sites.

$$
\mathrm{Dy}_{2} \mathrm{O}_{3} \stackrel{2 \mathrm{CdO}}{\longrightarrow} 2 \mathrm{D} y_{C d} \bullet+2 \mathrm{O}^{\times}+2 n+\frac{1}{2} \mathrm{O}_{2}(g)
$$

Given these known defect interactions, we can formulate the general trend of electronic and ther- 


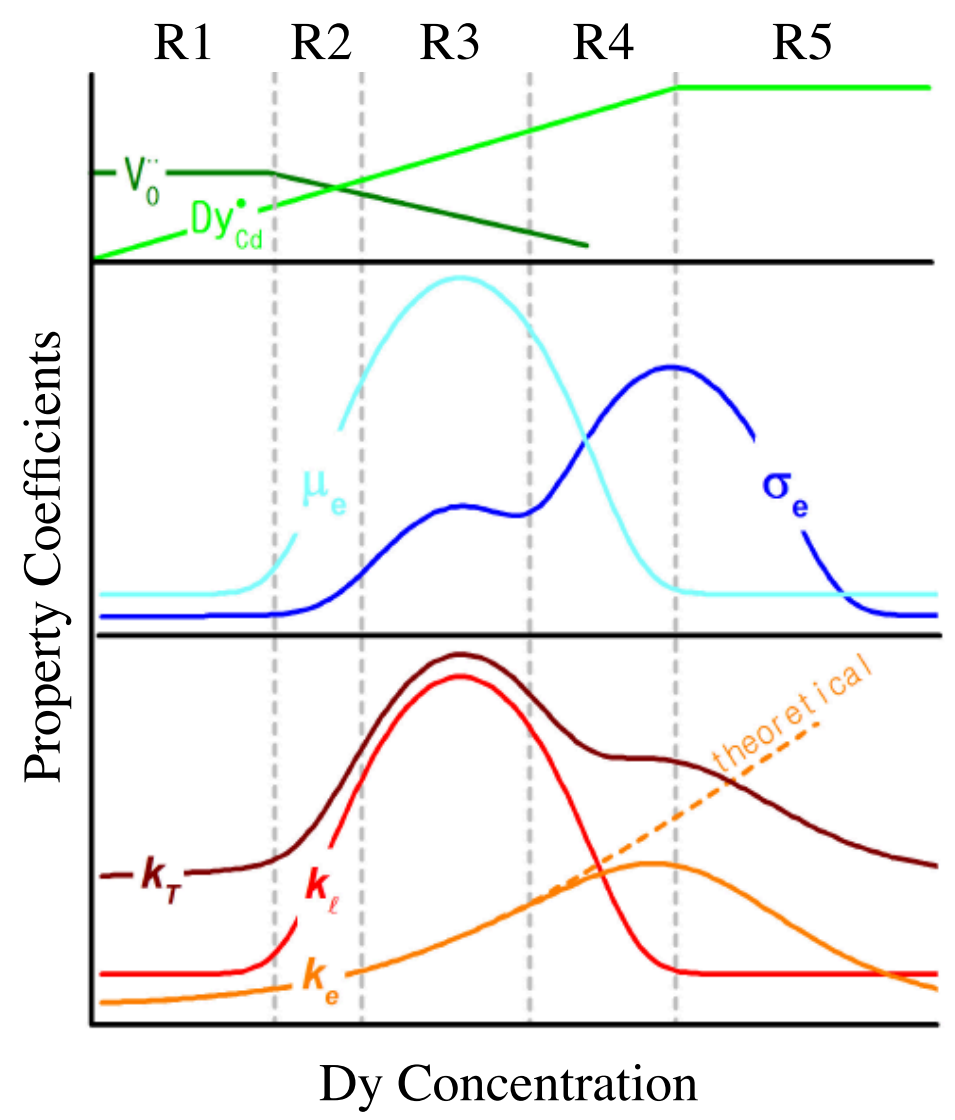

Figure 5.1: Qualitative illustration of the expected trend in electrical and thermal transport given interactions between increasing population of substitutional Dy defects and resulting decrease in oxygen vacancies [143].

mal transport with respect to Dy doping concentration. Published in the first of the two manuscripts associated with this work, Sachet et al. [143] illustrate these trends qualitatively in Fig. 5.1. In this conceptual representation, there are five major regions of varying transport.

The first region, labeled "R1", is the near intrinsic region, where transport is relatively unaffected compared to the baseline level of impurities and other point defects in the system. In the second region, "R2", the Dy impurities act to drive out the oxygen vacancies from the system. Replacing oxygen vacancy scattering centers with Dy substitutional impurities results in less overall scattering since the difference in radius of a Dy atom compared to a $\mathrm{Cd}$ atom is less than that of a vacant oxygen site compared to an occupied oxygen site, quantified in Table 4. This decrease in scattering results in an increase in both electronic and thermal mobility, thus increasing the respective conductivities. In addition, in this region we have started introducing a substantial population of electronic carriers since Dy is an electron donor, thus the electronic conductivity is expected to 
begin rising significantly. The third region, "R3", is where we start to see diminishing returns in thermal transport for the addition of Dy into the system. The substitutional defect concentration has now far exceeded the other defects in the system and the mobility can no longer increase due to scattering from the Dy dopants. While the thermal and electronic mobilities will begin to decrease in this region, the increasing dopant population will continue to introduce more free carriers into the system, thus continuing to increase the total electronic conductivity further than the peak in the mobility. Separating the phonon and electron contributions to thermal conductivity, we expect the phonon thermal conductivity to decrease significantly in this region while the electron thermal conductivity will trend with the electronic conductivity. In the fourth region, the electronic conductivity reaches its peak, no longer being able to rely on an increase in carrier population to make up for the decrease in mobility. Finally, in the fifth region, "R5", as we near the solubility limit for Dy in $\mathrm{CdO}$, all conductivities are expected to decrease from scattering of the large population of defects in the system.

\subsection{Modeling Phonon-Point Defect Interactions in CdO}

To quantify this impact on phonon scattering, I use the parameters for defect scattering time reviewed in Sect. 2.2

$$
\tau_{\text {Def }}^{-1}=A \omega^{4} x_{\text {Def }}\left[\left(\frac{\Delta M_{\text {Def }}}{M_{\text {Host }}}\right)^{2}+2\left[\left(\frac{\Delta G_{\text {Def }}}{G_{\text {Host }}}\right)-6.4 \gamma\left(\frac{\Delta \delta_{\text {Def }}}{\delta_{\text {Host }}}\right)\right]^{2}\right]
$$

where $A$ is a parameter determined by the lattice constant and phonon group velocity of the material, $\omega$ is the phonon frequency, and $x_{D e f}$ is the concentration of a given defect. The squared terms represent the scattering characteristics due to the mass change and strain change from a defect in the lattice. The mass difference is determined by the difference in mass of the defect from the host, $\Delta M_{D e f}$ divided by the average atomic mass of the host, $M_{D e f}$. The strain difference is determined using similar factors where $\Delta G_{D e f}$ is the change in bond stiffness, $\Delta \delta_{d e f}$ is the change in lattice parameter, and $\gamma$ is the Grüneisen parameter [168]. In the case of a vacancy, the factor accounting for the mass change takes the following form to account for potential energy differences with the absence of an atom [65] 
Table 4: Comparison of Factors Influencing Phonon Scattering Times in Dy:CdO

\begin{tabular}{c|c|c|c} 
Defect & $\frac{\Delta M_{\text {Def }}}{M_{\text {Host }}}$ & $\frac{\Delta G_{\text {Def }}}{G_{\text {Host }}}$ & $\frac{\Delta \delta_{\text {Def }}}{\delta_{\text {Host }}}$ \\
\hline \hline$D y_{C d} \bullet$ & 1.53 & -0.06 & 0.15 \\
\hline$V_{O}^{\bullet \bullet}$ & -2.24 & 1 & -0.67 \\
\hline
\end{tabular}

$$
\frac{\Delta M_{V a c}}{M_{\text {Host }}}=-\frac{M_{\text {Vac }}}{M_{\text {Host }}}-2
$$

where $M_{V a c}$ is the mass of the missing atom.

Given this formulation, the scattering between the defects in question can be compared by analyzing the $\Delta M_{D e f}, \Delta G_{D e f}$, and $\Delta \delta_{D e f}$ parameters for both types of defects, summarized in Table 4. The oxygen vacancy scattering is expected to be significantly higher for a given concentration compared to the Dy dopants.

Physically, this difference makes intuitive sense; the perturbation of an atom that is slightly larger but otherwise similar will be smaller than a site on the lattice where there should be a relatively large atom, but it is not present at all. The relaxation that will occur around this vacant site will then result in much different vibrational characteristics both from a perspective of the mass of this vibrating species and the strain field that will be present around the vacancy.

\subsection{Experimental Evidence of Phonon-Point Defect Interactions}

In order to observe this change experimentally, I measured the thermal conductivity of a series of thin films doped with Dy to isolate the role of phonon-vacancy and phonon-dopant scattering on thermal conductivity $[143,144]$. In addition, I compared the data to well-known theory and used these experimental findings to evaluate the accuracy of decade-old models for phonon-vacancy scattering effects on thermal conductivity [144].

I used time-domain thermoreflectance (TDTR) to measure the thermal conductivity of CdO thin films at room temperature and $80 \mathrm{~K}$ in order to experimentally identify the role of phonon-vacancy scattering on thermal conduction in $\mathrm{CdO}$. By the mechanism of the $80 \mathrm{~K}$ thermal conductivity measurements, I conclusively show that the behavior of the phonon and electron dominated thermal 
carrier regimes differ based on temperature, while the various point defect interactions obey relatively well-known trends exhibited in the literature [21, 81].

In these TDTR experiments, the metal transducer was $\mathrm{Al}$ with a thickness of $83 \pm 3 \mathrm{~nm}$, and the pump and probe spot radii were $42 \mu \mathrm{m}$ and $11 \mu \mathrm{m}$, respectively. I assume literature values for the heat capacity of the $\mathrm{Al}$ [279], $\mathrm{CdO}$ [297], $\mathrm{MgO}$ [298] and thermal conductivity of the $\mathrm{MgO}$ [62]. I fit for the thermal conductivity of the $\mathrm{CdO}$ layer and the thermal boundary resistance between the $\mathrm{Al}$ and $\mathrm{CdO}$. Given the relative magnitudes of the thermo-physical properties of the constituent materials and the modulation frequency of our heating event, we are not sensitive to the thermal boundary resistance between the $\mathrm{CdO}$ and the $\mathrm{MgO}$ substrate. The major source of error in the analysis arises from uncertainty in the thickness of the $\mathrm{Al}$ transducer. To minimize this uncertainty, I verified the Al film thickness with both X-ray reflectivity data (provided by Professor Maria's group at NC State) and picosecond acoustic analyses [246, 247].

In order to understand the physical mechanisms affecting the measured thermal conductivities in these materials, I conducted measurements at $80 \mathrm{~K}$ to compare to measurements at room temperature. Seen in Fig. 5.2, the room temperature data show distinct double peak characteristics, with local maxima in thermal conductivity occurring at Dy concentrations of $0.0182 \%$ and $0.083 \%$ [143]. This arises from the previously discussed interplay between various point defect populations and the introduction of sufficient electronic carriers to elevate the electronic contribution to the thermal conductivity to a significant level. In the low temperature data reported in this work, the electronic conductivity is reduced and the lattice thermal conductivity is enhanced. By comparing the $80 \mathrm{~K}$ measurements to the room temperature data, we observe clear signatures of the major contributions to the thermal conductivity over the different regimes of Dy doping, discussed below.

Three major regions of defect interaction are captured by the data shown in Fig. 5.2. Initially, in region "I" of Fig. 5.2, the addition of Dy results in a decrease in oxygen vacancies, thus decreasing the total scattering of thermal carriers and increasing the total thermal conductivity. In the second region, "II", the Dy concentration is high enough to significantly scatter the thermal carriers (i.e., both electrons and phonons) in the system and the only contribution acting to increase the thermal conductivity is the addition of free carriers from doping. The final data point in this region (0.08 at. $\%$ Dy) represents the maximum electrical contribution to the thermal conductivity, $\kappa_{\text {elec }}$, which leads to a second peak in the measured thermal conductivity at room temperature. In the final region, 

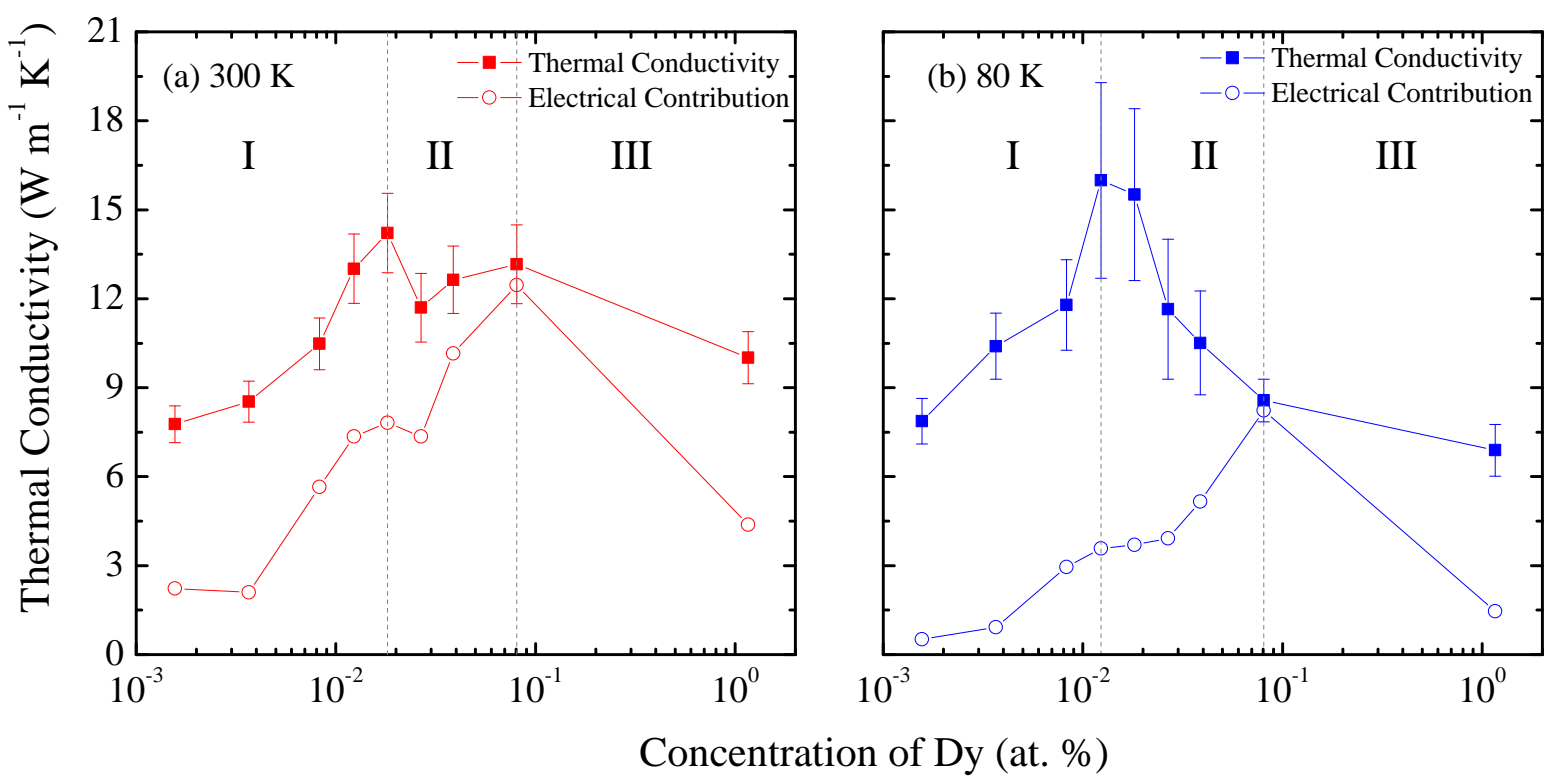

Figure 5.2: Measured thermal conductivity of CdO:Dy for various Dy concentrations. Markers and lines indicate total measured thermal conductivity and calculated electronic contribution to thermal conductivity, respectively. Measurements were taken at (a) $300 \mathrm{~K}$ and (b) $80 \mathrm{~K}$.

"III", the Dy concentration is high enough to effectively scatter all carriers and the total thermal conductivity decreases. This final region includes data from near the solubility limit of Dy in CdO, giving us an upper bound on the defect interactions, as we expect formation of secondary phases at this concentration.

In Fig. 5.2b, there are clear differences in the trends in thermal conductivity as a function of Dy concentration. This variation of thermal characteristics with temperature is crucial to understanding the dominant thermal transport mechanisms being affected in each region. Here, region "I", delineated by the total peak in the thermal conductivity, has shifted, which can be understood by a change in the phonon population (at low temperatures we expect less high frequency phonons which are more impacted by point defect scattering). The increase in the magnitude of this peak arises from a reduction in phonon-phonon scattering at low temperatures.

At $80 \mathrm{~K}$, the second peak that is present at room temperature, which arises from increase in electronic conductivity, has significantly diminished; this is due to the decrease in the electrical contribution to thermal conductivity at $80 \mathrm{~K}$ relative to room temperature. Included in this fig- 
ure is the calculated electronic contribution to thermal conductivity, $\kappa_{\text {elec}}$, determined using the Wiedemann-Franz law applied to our electrical resistivity measurements on these samples [143]. The Wiedemann-Franz law, which holds well for electrical conductors, states that the electrical contribution to the thermal conductivity, $\kappa_{e l}$ at a given temperature, $T$, is proportional to the electrical conductivity, $\sigma$ by a constant known as the Lorenz number, $L$ [129].

$$
\begin{aligned}
\kappa_{e l} & =\sigma L T \\
L & =2.44 \times 10^{-8} W \Omega K^{-2}
\end{aligned}
$$

To understand the effects of defect population modulation on the phonons in the system, the electronic contribution is subtracted from the total measured thermal conductivity and only the phonon thermal conductivity is analyzed, modeled by $\kappa_{p h}=1 / 3 C_{v} v^{2} \tau$, where $C_{v}$ is the heat capacity, $v$ is the phonon velocity, and $\tau$ is the overall scattering time given in Eq. 141. In this material system, we expect contributions to the phonon thermal resistance from anharmonic interactions, $\tau_{P h}$, natural impurity scattering, $\tau_{I m p}$, boundary scattering, $\tau_{\text {Bound }}$, dopant scattering, $\tau_{D e f}$, and vacancy scattering, $\tau_{\text {Vac }}$. This is combined via Matthiessen's rule, given by

$$
\frac{1}{\tau}=\frac{1}{\tau_{P h}}+\frac{1}{\tau_{I m p}}+\frac{1}{\tau_{\text {Bound }}}+\frac{1}{\tau_{D e f}}+\frac{1}{\tau_{V a c}}
$$

I used literature data to determine the heat capacity and phonon velocities as a function of wavelength from the acoustic branches of the phonon dispersion curve [299]. In the model for this study, I use a polynomial fit to the acoustic phonon branches of $\mathrm{CdO}$, seen in Fig. 5.3, and follow the modeling approach outlined in Sect. 2.2. I assume a spherical Brillouin zone with a radius of $k=\frac{2 \pi}{a}$, taken in the [100] direction. This corresponds to the rocksalt crystal structure, where the plane of symmetry that dictates the Brillouin zone edge is located $1 / 2$ of a lattice constant from the origin.

The data obtained in this study is then used to determine the impact and form of the major phonon scattering mechanisms introduced by doping $\mathrm{CdO}$ with Dy. Since the optical branches have a very low corresponding group velocity, the associated contributions to the thermal conductivity are negligible, and I ignore them in the model. 


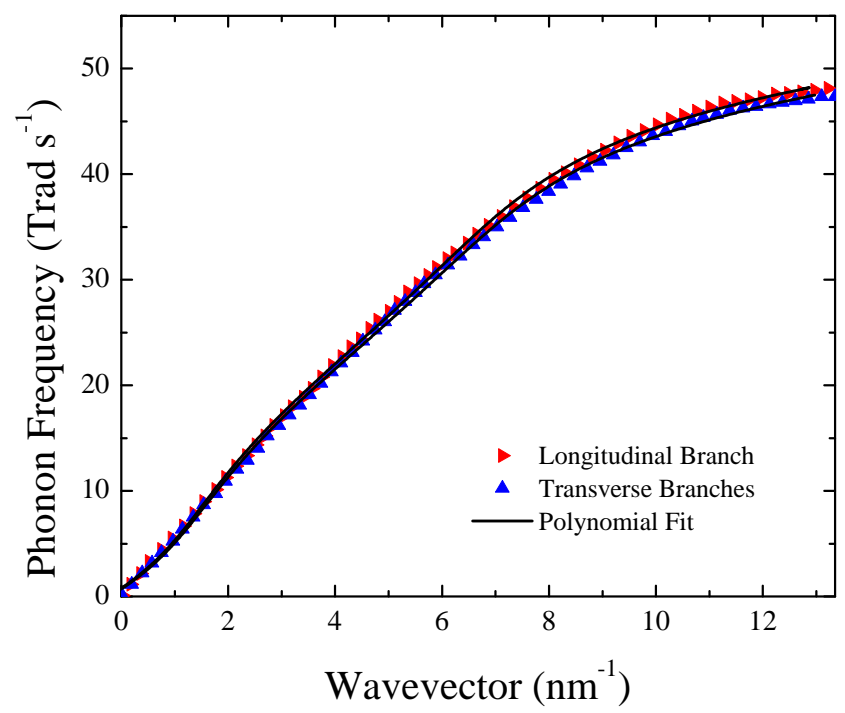

Figure 5.3: Literature values of acoustic phonon dispersion [299] for longitudinal (red) and transverse (blue) phonon polarizations along with the polynomial fits (black) used for the model of thermal transport in CdO.

The baseline scattering mechanisms, which are assumed to be constant with doping, are phononphonon scattering, natural impurity scattering, and film boundary scattering. To this extent I calibrate the scattering parameters using temperature dependent literature data for phonon-phonon scattering [300] and the undoped $\mathrm{CdO}$ film of our sample set for baseline impurity and film boundary scattering. I measured the temperature dependent thermal conductivity of the undoped sample and used these measurements, found in Fig. 5.4 along with the fitted model, to determine the baseline impurity and boundary scattering parameters.

Using the previously discussed formalism for defect scattering, I modeled the thermal conductivity of the measured $\mathrm{CdO}$ films given the known concentration of Dy dopants and assuming a concentration of oxygen vacancies based off of the electrical conductivity of the undoped films (estimated to be $2 \times 10^{19} \mathrm{~cm}^{-1}$ ) that decays an order of magnitude due to the substitutional Dy doping [143]. This initial model assumes the major changing defects in the films are only the Dy dopants and the oxygen vacancies.

This model, labeled as the "Dy Dominated" model in Fig. 5.5, captures the region "I" data rather well. The data shown in Fig. 5.5 is the measured thermal conductivity with the calculated electrical contribution subtracted off, leaving only the phonon thermal conductivity. It is interesting to note in 


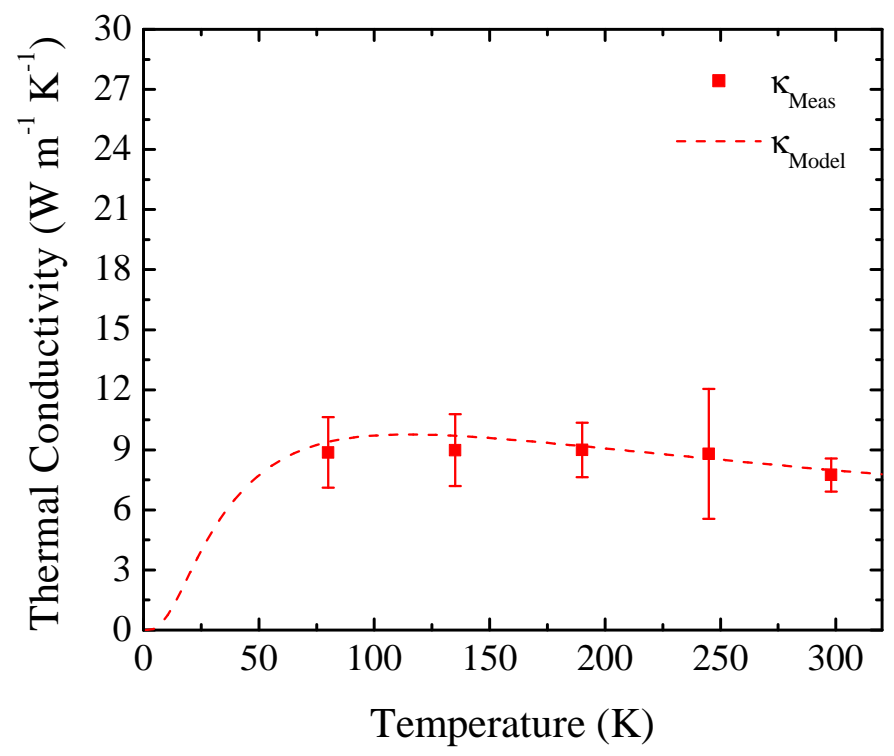

Figure 5.4: Measured thermal conductivity as a function of temperature of the undoped film for calibration of the model of thermal conductivity of $\mathrm{CdO}$ thin films (dashed line).

this model that the contribution to the thermal conductivity from the change in vacancy population is relatively low; neglecting oxygen vacancies all together results in a $25 \%$ difference in the thermal conductivity at low Dy concentrations, and the difference drops to less than $10 \%$ once the doping level exceeds $10^{-2}$.

The Dy dominated model fails to capture the data for the phonon thermal conductivity in the higher Dy doping concentrations (region "II"). Literature findings suggest that in highly doped oxide systems, substitutional dopants can be compensated by other vacancy mechanisms in order to reduce the overall energy of the system [301, 302]. In accordance with these findings, I investigated the phonon scattering effects of $\mathrm{Cd}$ vacancies with populations dictated by the substitutional doping concentration; for these calculations I rely on the model given in Eq. 137. This model, referred to in Fig. 5.5 as the "Vacancy Compensated" model agrees with the measured data significantly better at the higher Dy concentrations than the "Dy Dominated" model. This agreement, along with similar findings in other oxide systems found in the literature, serves to indicate that there is an introduction of other point defects compensating the Dy dopants at these high doping concentration levels, and it is these compensating defects that drastically reduce the phonon thermal conductivity.

The highest doping concentration of the measured data set does not follow this continued down- 


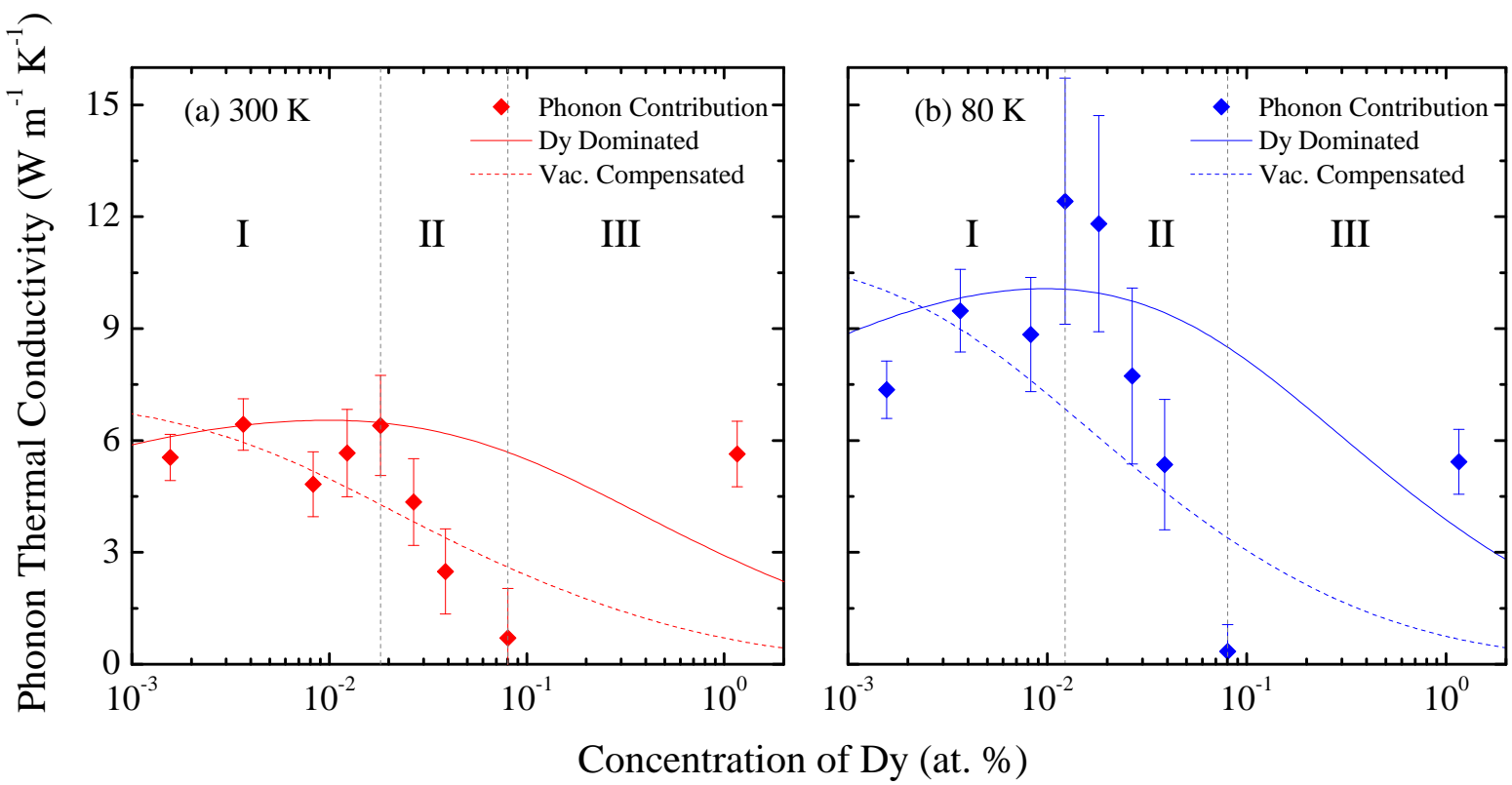

Figure 5.5: Phonon thermal conductivity of CdO:Dy at (a) $300 \mathrm{~K}$ and (b) $80 \mathrm{~K}$ along with bounding models for a system dominated by scattering from Dy doping (solid lines) and a model for a system with additional scattering from vacancies with concentrations pinned by the Dy concentration (dashed lines).

ward trend in the phonon thermal conductivity. This is easily understood when considering that this sample has exceeded the solubility of $\mathrm{Dy}$ in $\mathrm{CdO}$ and our characterization indicates that secondary phases have formed [143]. In this case, it is likely that there are multiple regions that will behave more similarly to dilute solutions of Dy in $\mathrm{CdO}$ rather than highly doped solutions. This hypothesis is supported by the relative agreement with the "Dy Dominated" model compared to the "Vacancy Compensated" model. Further characterization of the precipitated phases and their respective Dy concentrations would need to be carried out in order to verify this hypothesis, but is beyond the scope of this study.

There are a number of other components that could be at play to dictate the phonon thermal conductivity of these films such as minority defect populations, contributions to the thermal conductivity from optical phonons, and phonon drag from the free electrons in the system [160]. However, the agreement between the relatively simple models and the data measured, suggest that we have identified the major phonon scattering mechanisms in these materials. Furthermore, the agreement among the measured data in the various regimes of Dy doping suggests that the phonon thermal 
conductivity, and analytical modeling thereof, can be used to identify signs of complex defect interactions in these functional oxides. The presentation of both models, one taking into account only the known defects, and the other identifying the remaining scattering sites, serves as bounds for the major phonon scattering mechanisms in this material and significantly aids in the understanding of the phonon-related thermal conductivity tuning of CdO using Dy dopants.

In conclusion, the CdO:Dy material system illustrates intricate bi-directional tunability of thermal conductivity in complex oxide materials, offering potential next steps for thermal engineering using functional oxides. I have shown that with the introduction of Dy dopants, the thermal conductivity of $\mathrm{CdO}$ can be increased or decreased via the interplay among the electronic contribution to thermal conductivity and thermal carrier scattering due to point defect and vacancy concentrations present in the system. Decreasing the intrinsic oxygen vacancy concentrations increases the electronic mobility, leading to an increase in thermal conductivity at low Dy doping levels, but has a limited effect on the phonon thermal conductivity. At higher Dy doping levels, the lattice thermal conductivity is impacted. Using measurements at multiple temperatures, I have formulated a model for the phonon thermal conductivity that highlights the major scattering mechanisms present in the system including mass-impurity and vacancy scattering. I have shown that Dy mass-impurities contribute to thermal scattering at low Dy concentrations, as expected. However, at higher Dy concentrations, additional defects, namely cation vacancies, must be taken into account to capture the trend in the data. This results in models which act as bounds for the measured data as the system transitions from dilute dopant scattering, to scattering due to both dopant and vacancies at high Dy concentrations. 


\section{$6 \quad$ Phonon Interactions with Intrinsic Point Defects in $\mathrm{TiO}_{2}$}

In this chapter, I will concentrate on the interaction of phonons with intrinsic point defects. Control of intrinsic defect concentrations is achieved via annealing of bulk single crystals under various partial pressures of oxygen. Using TDTR, I measure various samples with a range of defect concentrations to determine the impact of point defect scattering on the thermal conductivity. This study will provide insight into the changing thermal properties during the dielectric breakdown process.

The material system of choice for this study is single crystal $\mathrm{TiO}_{2}$. As $\mathrm{TiO}_{2}$ is an oxide that has been studied for many years, there is a substantial collection of literature to refer to with respect to defect dynamics and resulting properties. The two most common forms of $\mathrm{TiO}_{2}$ are anatase and rutile. In this study, I will only concentrate on the rutile crystal structure, a body-centered tetragonal structure with titanium cations on the corners and body center position, and oxygen anions forming octahedra around each cation, as seen in Fig. 6.1.

\subsection{Applications and Background of $\mathrm{TiO}_{2}$}

Common applications of $\mathrm{TiO}_{2}$ range from a wide variety of optical applications to electronic applications. It is used as an ultraviolet radiation absorber in sunscreen [303] as well as a pigment for white paints, plastics, paper, and cosmetics [304-306]. $\mathrm{TiO}_{2}$ also has some very interesting photocatalytic behavior, leading to its use as photo-induced catalyst for chemical application as well as in photovoltaic applications [47, 307-309]. Nano-structured $\mathrm{TiO}_{2}$ particles, nanotubes, and nanoporous membranes are a key component to the operation of dye-sensitized solar cells, where the photoinduced electron-hole splitting occurs at the nano-structured $\mathrm{TiO}_{2}$ surface [310-312]. $\mathrm{TiO}_{2}$ is also used in optics, for example as a multi-layer dielectric mirror for laser line applications [313-315].

The electronic applications of $\mathrm{TiO}_{2}$ are particularly relevant to this study. As mentioned in Ch. 2.5, the major motivating application for the following experiment is based around understanding thermal transport during dielectric degradation.

Dielectric degradation and breakdown are major reliability issues in microelectronic devices [216, 317]. Since thermal buildup can significantly accelerate dielectric breakdown, understanding the 


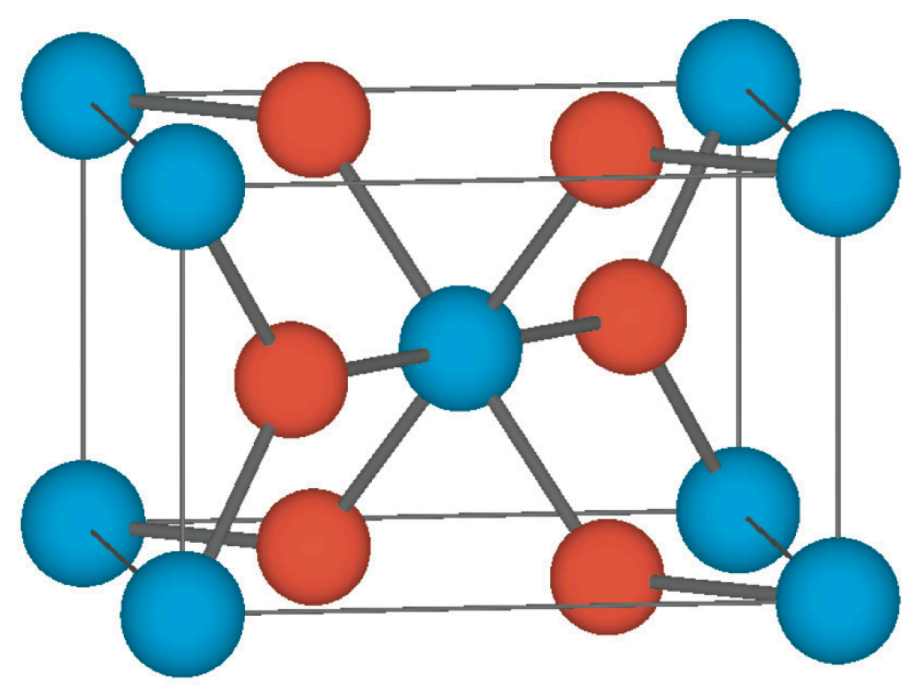

Figure 6.1: The unit cell of rutile $\mathrm{TiO}_{2}$ with $\mathrm{Ti}$ cations in blue and $\mathrm{O}$ anions in orange [316].

evolution of the intrinsic thermal properties of dielectric materials under prolonged electric fields is of critical importance to addressing this failure mechanism [209, 210].

With increased concentration of point defects, thermal carrier propagation will be disrupted and local hotspots may lead to increased probability of device failure [212, 215, 224]. In the region of the electrodes of a dielectric material under prolonged electric fields, point defect concentrations can increase well beyond initial, homogeneous concentrations and lead to highly defective layers that can transition into entirely new phases from the severe non-stoichiometry [197, 318-321].

The impact of this defect buildup on thermal transport can be assessed in a systematic way by analyzing systems with similar levels of homogenous defect concentrations. The literature clearly outlines the general trend of reduced phonon transport with high point defect concentrations in a crystal $[78,168]$. The analytical form of this increase in phonon scattering with point defects has been previously determined not only for elemental crystals, but for oxides as well [65, 80]. However, a systematic experimental study that takes into account the full series of equilibrium point defects and their effect on thermal conductivity in a dielectric crystal has yet to be demonstrated.

Control over defect profiles in oxides can be accomplished using control over the partial pressure of oxygen during annealing to dictate oxygen activity and alter relative equilibrium concentrations of point defects [53]. This technique can be used to fabricate single crystals with point defect 
concentrations that span the range of the variable defect concentrations found within a dielectric during degradation and breakdown. The information gleaned from this study can then be used to model the evolution of thermal transport under prolonged electric fields and determine the threshold at which the local thermal conductivity will have an impact on thermal runaway and dielectric breakdown.

In $\mathrm{TiO}_{2}$, defect accumulation has led to Magnéli phase formation at electrodes, indicating a significant change in stoichiometry driven by the addition of $\mathrm{Ti}^{3+}$ interstitials, which are known to be the mobile defects at room temperature [197]. The non-stoichiometry of this defected region, which has a thickness on the order of $200 \mathrm{~nm}$, is likely in the defect concentration range of $0.5-1$ at. $\%$.

This work aims to experimentally elucidate the impact of defect concentration on thermal transport systematically by manipulating bulk point defect concentrations in rutile $\mathrm{TiO}_{2}$. I use analytical models to further understand the trends in thermal transport and identify the threshold of the impact of defectivity on the thermal conductivity as well as the impact of new phase formation at very high defect concentrations. These findings will help serve as a guide for process conditions not only in the dielectric breakdown process, but in general dielectric fabrication as well.

\subsection{Measurement and Control of Reduced $\mathrm{TiO}_{2}$}

Using TDTR, I measure the change in thermal conductivity of rutile single crystals that have been processed to contain increasing concentrations of intrinsic point defects. In this study, the metal film used as a thermal transducer is $65 \mathrm{~nm}$ of $\mathrm{Al}$ deposited with electron-beam evaporation onto the crystal of interest. I use literature values of the volumetric heat capacities [298] and verify the thermal conductivity of the $\mathrm{Al}$ with a four point probe measurement. As the main source of error in the analysis arises from variation in the thickness of the Al, I use both picosecond acoustics and profilometry to measure a thickness of at $64 \pm 3 \mathrm{~nm}$.

The penetration depth of our thermal measurement is dependent on the frequency of the modulated pump heating event. The relationship between the $1 / e$ decay in the thermal gradient, the thermal properties of the material being studied, and the modulation frequencies is 


$$
d_{\text {therm }}=\sqrt{\frac{k}{\pi f C}}
$$

where $d_{\text {therm }}$ is the thermal penetration depth, $k$ is the thermal conductivity, $C$ is the volumetric heat capacity, and $f$ is the modulation frequency. In order to verify that the thermal measurements that we conduct are not dictated by the near-surface nature of our measurement, we probe various thermal penetration depths to ensure that there is no depth dependence in the measured thermal conductivity of the crystals. The modulation frequencies used range from $8.8 \mathrm{MHz}$ to $500 \mathrm{kHz}$, corresponding to thermal penetration depths in $\mathrm{TiO}_{2}$ ranging roughly from $300 \mathrm{~nm}$ to $1.3 \mu \mathrm{m}$.

The crystals were purchased from the MTI corporation and are $10 \mathrm{~mm} \times 10 \mathrm{~mm} \times 0.5 \mathrm{~mm}$ polished (100)-oriented and (001)-oriented rutile $\mathrm{TiO}_{2}$. Annealing was done both at the University of Virginia as well as at North Carolina State University in collaboration with Professor Dickey. We annealed each crystal at $1100^{\circ} \mathrm{C}$ for $36 \mathrm{~h}$ at varying partial pressures of oxygen $\left(p \mathrm{O}_{2}\right)$ in order to control the defect concentrations. In order to precisely control the $p \mathrm{O}_{2}$, we varied the flow rate of an $\mathrm{Ar} / \mathrm{H}_{2}$ forming gas mixture in the annealing furnace. Utilizing the interlock system at NCSU, we quenched the crystals in the forming gas environment to room temperature using a sealed interlock. We varied the $\mathrm{pO}_{2}$ during the anneal to range from $1.7 \times 10^{-12}$ to $8.4 \times 10^{-18} \mathrm{~atm}$.

Using literature values for the equilibrium mass action constants of the major intrinsic defects in $\mathrm{TiO}_{2}[302,309,322]$, I determined the concentration of various species of point defects dictated by the environmentally controlled anneals. The major populations of point defects present are oxygen vacancies, titanium interstitials, and titanium vacancies. The calculated concentrations of these defects, along with the concentrations of the electrons and holes can be seen in Fig. 6.2, calculated at $1100^{\circ} \mathrm{C}$ with an $\mathrm{Al}$ impurity concentration of $5 \times 10^{-5}$. The defect concentrations from this calculation will be used in the model for the phonon thermal conductivity to predict the change in phonon scattering by point defects in $\mathrm{TiO}_{2}$.

As discussed in Sect. 2.4, I expect the sub-stoichiometry of the reduced $\mathrm{TiO}_{2}$ to be severe enough to induce the ordering of point defects and form Magnéli phases. This will be the largest contribution to any departures from the model that is based on concentrations of homogeneously distributed atomic scale phonon scattering centers. 


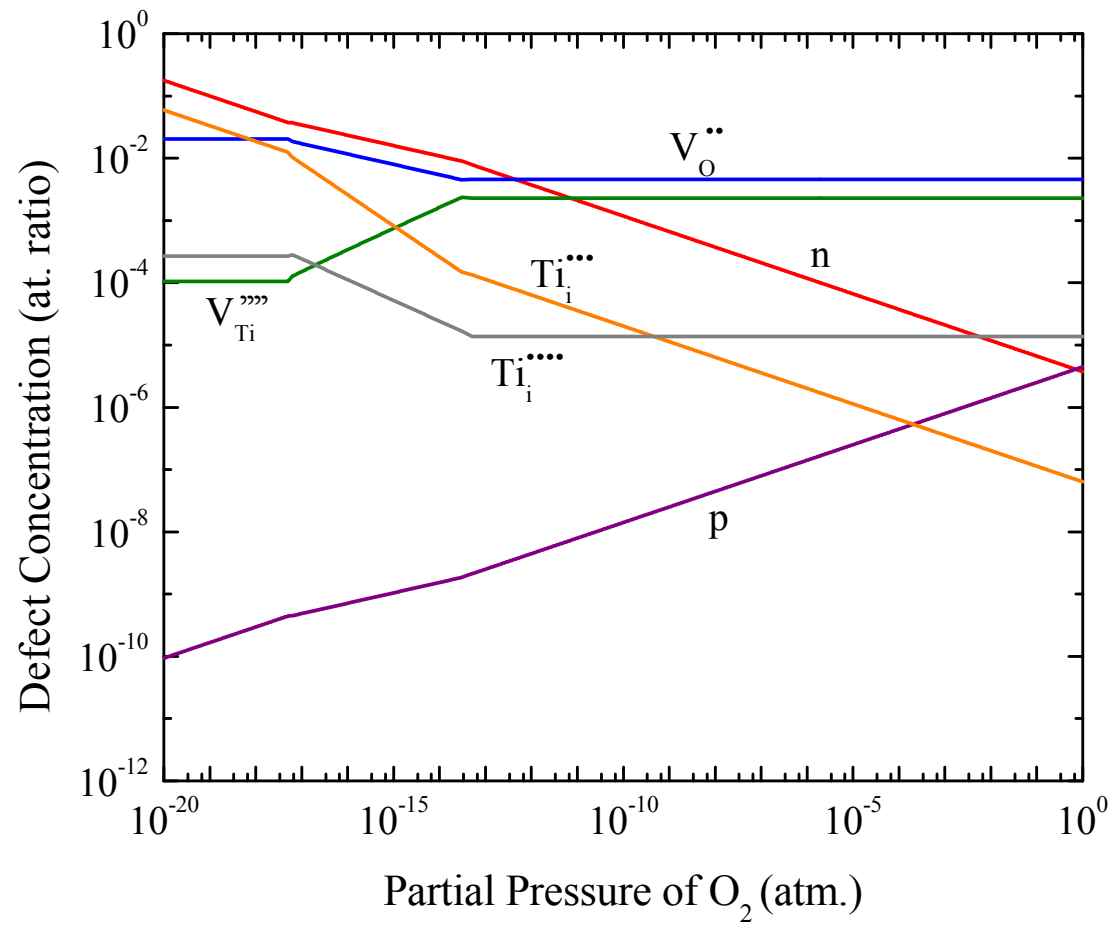

Figure 6.2: Concentration of primary point defects in $\mathrm{TiO}_{2}$ dictated by partial pressure of oxygen in $1100{ }^{\circ} \mathrm{C}$ anneal.

\subsection{Modeling Phonon Transport in Rutile $\mathrm{TiO}_{2}$}

In order to further understand the trends in the thermal conductivity of defected functional oxides, I developed an analytical model for the phonon thermal conductivity of $\mathrm{TiO}_{2}$. The model consists of an integration over phonon wave vectors for each branch in the phonon dispersion. Outlined previously, the thermal conductivity, $\kappa$, is the product of the heat capacity, $C$, the phonon group velocity, $v$, and the characteristic scattering time, $\tau$.

$$
\kappa=\sum_{i} \int C v^{2} \tau d k
$$

In the model used in this study, all of these components are modeled as phonon wave vector, $k$, dependent with relationships dictated by the phonon dispersion relationship for each branch, $i$. The rutile unit cell is made up of a tetragonal structure with a six point basis, with titanium on the $(0,0,0)$ and $(1 / 2,1 / 2,1 / 2)$ sites and oxygen on the $(1 / 4,1 / 4,0),(3 / 4,3 / 4,0),(1 / 4,3 / 4,1 / 2)$, and $(3 / 4,1 / 4,1 / 2)$ sites resulting in 18 total branches (3 acoustic and 15 optical). Since rutile is anisotropic, we must take into account both the $\Gamma \rightarrow \mathrm{X}$ and $\Gamma \rightarrow \mathrm{Z}$ directions of the Brillouin zone 


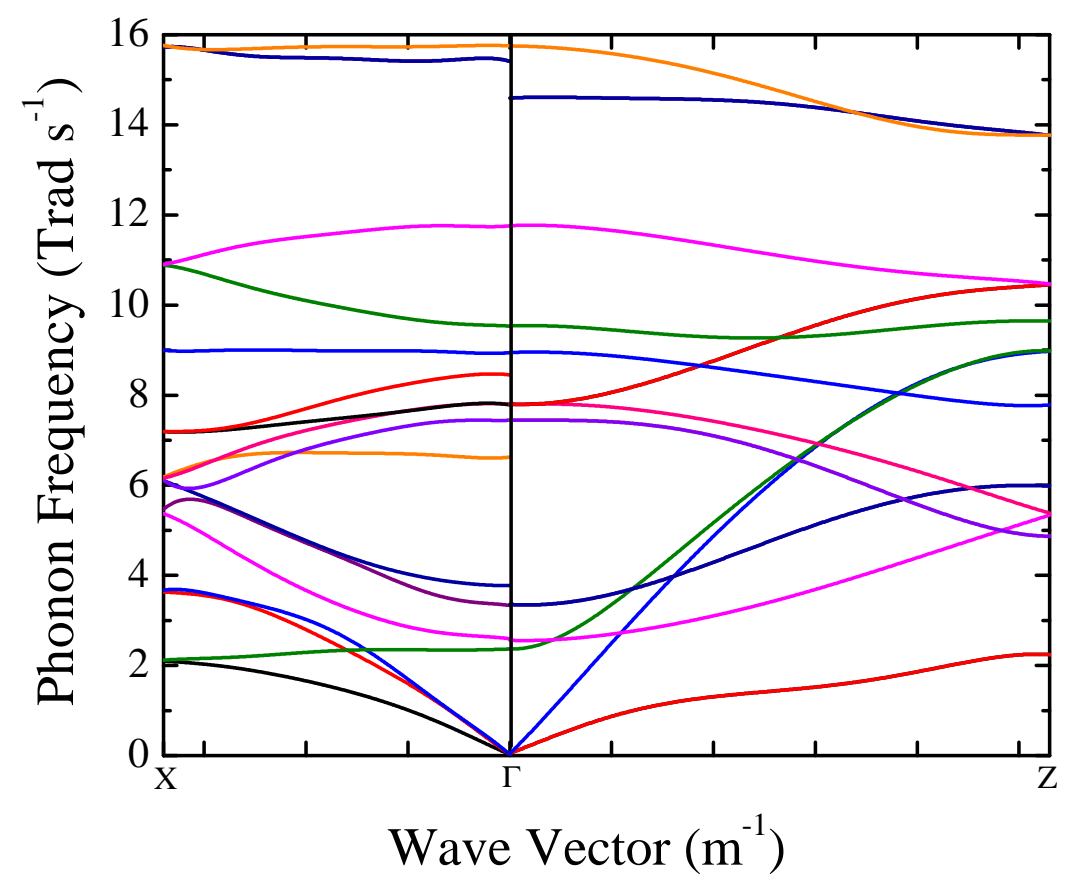

Figure 6.3: Phonon dispersion in the $\Gamma \rightarrow \mathrm{X}$ and $\Gamma \rightarrow \mathrm{Z}$ directions compiled from theoretical and experimental findings $[154,323]$.

when considering the dispersion diagram. As explained in the discussion below, in this case, I make an assumption of a cylindrical Brillouin zone instead of the typical spherical Brillouin zone which is assumed for an isotropic system. I fit the phonon dispersion which has been determined using lattice dynamics [154] using a fifth order polynomial for each branch, the result of which can be seen in Fig. 6.3.

The assumption of a cylindrical Brillouin zone leads to a definition of the phonon frequency, $\omega$, which depends on the cartesian wave vector relationships defined by the dispersion diagram as follows

$$
\omega=\omega\left(\sqrt{k_{x}^{2}+k_{y}^{2}+k_{z}^{2}}\right)
$$

where $k_{x}, k_{y}$, and $k_{z}$ are the phonon wave vectors in the $\Gamma \rightarrow X, \Gamma \rightarrow Y$, and $\Gamma \rightarrow Z$ direction, respectively. In the case of a cylindrically symmetric Brillouin zone the $\Gamma \rightarrow X$ and $\Gamma \rightarrow Y$ directions will be identical and Eq. 144 becomes 


$$
\omega=\omega\left(\sqrt{2 k_{x}^{2}+k_{z}^{2}}\right)
$$

As outlined in Ch. 2.2, the general formulation for the heat capacity of a crystal can be written as

$$
C=\frac{1}{8 \pi^{3}} \int_{k_{x}} \int_{k_{y}} \int_{k_{z}} \hbar \omega \frac{d F}{d T} d k_{x} d k_{y} d k_{z}
$$

where $\hbar \omega$ is the phonon energy dictated by the product of Planck's constant and the phonon frequency, and $\frac{d F}{d T}$ is the change in the Bose-Einstein distribution with temperature. I have defined this previously for a spherical Brillouin zone where

$$
\begin{aligned}
C & =\frac{1}{8 \pi^{3}} \int_{0}^{2 \pi} \int_{0}^{\pi} \int_{k} \hbar \omega \frac{d F}{d T} k^{2} \phi \sin (\theta) d k d \phi d \theta \\
& =\frac{1}{4 \pi^{2}} \int_{k} \hbar \omega \frac{d F}{d T} k^{2} d k
\end{aligned}
$$

where the only spatial term that dictates the heat capacity is the radius of the Brillouin zone, or the phonon wave vector.

In a tetragonal crystal, I approximate the Brillouin zone by a cylinder and the heat capacity is defined by

$$
C=\frac{1}{8 \pi^{3}} \int_{0}^{2 \pi} \int_{k_{r}} \int_{-k_{z}}^{k_{z}} \hbar \omega \frac{d F}{d T} k_{r} \phi d k_{z} d k_{r} d \phi
$$

where $k_{z}$ is the wave vector associated with the anisotropic axis, [001] in the case of $\mathrm{TiO}_{2}$, and $k_{r}$ is the wave vector in the isotropic plane, formulated as $k_{r}=\sqrt{k_{x}^{2}+k_{y}^{2}}$ where $k_{x}$ and $k_{y}$ are the wave vectors in the [100] and [010] directions in $\mathrm{TiO}_{2}$. By symmetry, this simplifies to

$$
C=\frac{1}{2 \pi^{2}} \int_{k_{r}} \int_{k_{z}} \hbar \omega \frac{d F}{d T} k_{r} d k_{z} d k_{r}
$$

The other components of the thermal conductivity are the phonon group velocity, which is determined by the first derivative of the phonon frequency with respect to wave number, and the phonon 
scattering time. Thus for each branch of the dispersion in the crystallographic direction of interest, the thermal conductivity in the anisotropic system is

$$
k_{i}=\frac{1}{2 \pi^{2}} \int_{k_{r}} \int_{k_{z}} \hbar \omega \frac{d F}{d T} v_{g}^{2} \tau k_{r} d k_{z} d k_{r}
$$

where the group velocity, $v_{g}=\partial \omega / \partial k$ is taken in the direction of transport. Thus if I am modeling the thermal conductivity in the [100] direction, I would use the group velocity dictated by the phonon dispersion in the $\Gamma \rightarrow X$ direction. The total phonon scattering time is modeled using Matthissein's rule, taking into account the following scattering times

$$
\frac{1}{\tau}=\frac{1}{\tau_{P h}}+\frac{1}{\tau_{\text {Imp }}}+\frac{1}{\tau_{\text {Bound }}}+\frac{1}{\tau_{\text {Instl }}}+\frac{1}{\tau_{\text {Vac }}}
$$

where $\tau_{P h}$ is the phonon-phonon scattering time, $\tau_{I m p}$ is the scattering of phonons with impurities, $\tau_{\text {Bound }}$ is the phonon-boundary scattering time, and the final components are the scattering of phonons with intrinsic point defects such as interstitials, $\tau_{\text {Instl }}$, and vacancies, $\tau_{V a c}$.

In this experiment, I assume that the first three scattering mechanisms are constant within the sample set and have a functional form as follows

$$
\frac{1}{\tau_{\text {Baseline }}}=\mathcal{A} T \omega^{2} \exp \left(\frac{-\mathcal{B}}{T}\right)+\mathcal{C} \omega^{4}+\frac{v}{d}
$$

where $T$ is the sample temperature, $d$ is the sample thickness, and $\mathcal{A}, \mathcal{B}$, and $\mathcal{C}$ are fitting parameters. We determine $\mathcal{A}$ and $\mathcal{B}$ using a least squares fit of literature values of measured thermal conductivity over a wide temperature range [15] and modify $\mathcal{C}$ to fit the measured values of the control sample in our specific sample set.

The formulation for scattering from known concentrations of point defects takes the form of Rayleigh scattering $\left(\propto \omega^{4}\right)$ and is determined by mass differences, size differences, and bond strength differences on the atomic level $[78,81,156,168]$ as

$$
\frac{1}{\tau_{D e f}}=\frac{a^{2} c}{4 \pi v_{g} v_{p}^{2}} x_{D e f} \omega^{4}\left[\left(\frac{\Delta M}{M}\right)^{2}+2\left[\left(\frac{\Delta G}{G}\right)-6.4 \gamma\left(\frac{\Delta \delta}{\delta}\right)\right]^{2}\right]
$$

where $a^{2} c$ is the tetragonal unit cell volume, $x_{D e f}$ is the atomic concentration of the defect of interest, $\gamma$ is the Grüneisen parameter, and $\Delta M / M, \Delta G / G$, and $\Delta \delta / \delta$ are the changes in mass, shear strength, 
and atomic radius, respectively, of the defect compared to the host. In this equation, the denominator of the prefactor contains the product of the group velocity, $v_{g}$, and the phase velocity, $v_{p}=\omega / k$, which is also directionally dependent. This is an important addition particularly in this model since we are considering contributions from the optical phonon branches in the full dispersion. Optical phonon branches will have a relatively low group velocity, but may have a much higher phase velocity.

In the case of a substitutional or interstitial defect, the mass difference parameter takes the form

$$
\frac{\Delta M}{M}=\frac{M_{D e f}-\bar{M}_{H o s t}}{\bar{M}_{H o s t}}
$$

where $M_{D e f}$ is the mass of the defect and $\bar{M}_{\text {Host }}$ is the average atomic mass of the host. In the case of a vacancy, this takes the form [80]

$$
\frac{\Delta M_{V a c}}{M}=-\frac{M_{V a c}}{\bar{M}_{\text {Host }}}-2
$$

where $M_{V a c}$ is the mass of the vacant atom.

In the case of the interstitial defects, the change in radius is determined by the difference between the radius of the interstitial site in rutile $\mathrm{TiO}_{2}$ [316] and the radius of the anion [324].

The values used for each type of defect accounted for in this model were taken from literature [324-326]. The impact of intrinsic defects on the thermal conductivity of rutile $\mathrm{TiO}_{2}$ can be seen in Fig. 6.4 for a wide range of concentrations of titanium interstitials $\left(\mathrm{Ti}^{3+}\right.$ and $\mathrm{Ti}^{4+}$ ), titanium vacancies, and oxygen vacancies (as well as a simple linear summation of all four). Comparing this model to the results from our measurements, we can use inputs determined by the equilibrium concentration at the anneal temperature, seen in Fig. 6.2, and determine the expected impact of intrinsic defects in highly reduced $\mathrm{TiO}_{2}$ on thermal transport.

The model of the thermal conductivity dependence on point defect concentration allows us to better understand the impact from each type of defect as the $\mathrm{pO}_{2}$ changes. In terms of thermal resistances, the phonon scattering times can be combined as thermal resistors in series. As this is the case, when deconvolving the effects of various scattering mechanisms, it is useful to analyze the thermal resistivity instead of its inverse, the thermal conductivity.

The contribution of each type of defect scattering mechanism can be computed by considering only one type of point defect at a time (at a concentration dictated by the equilibrium concentration) 


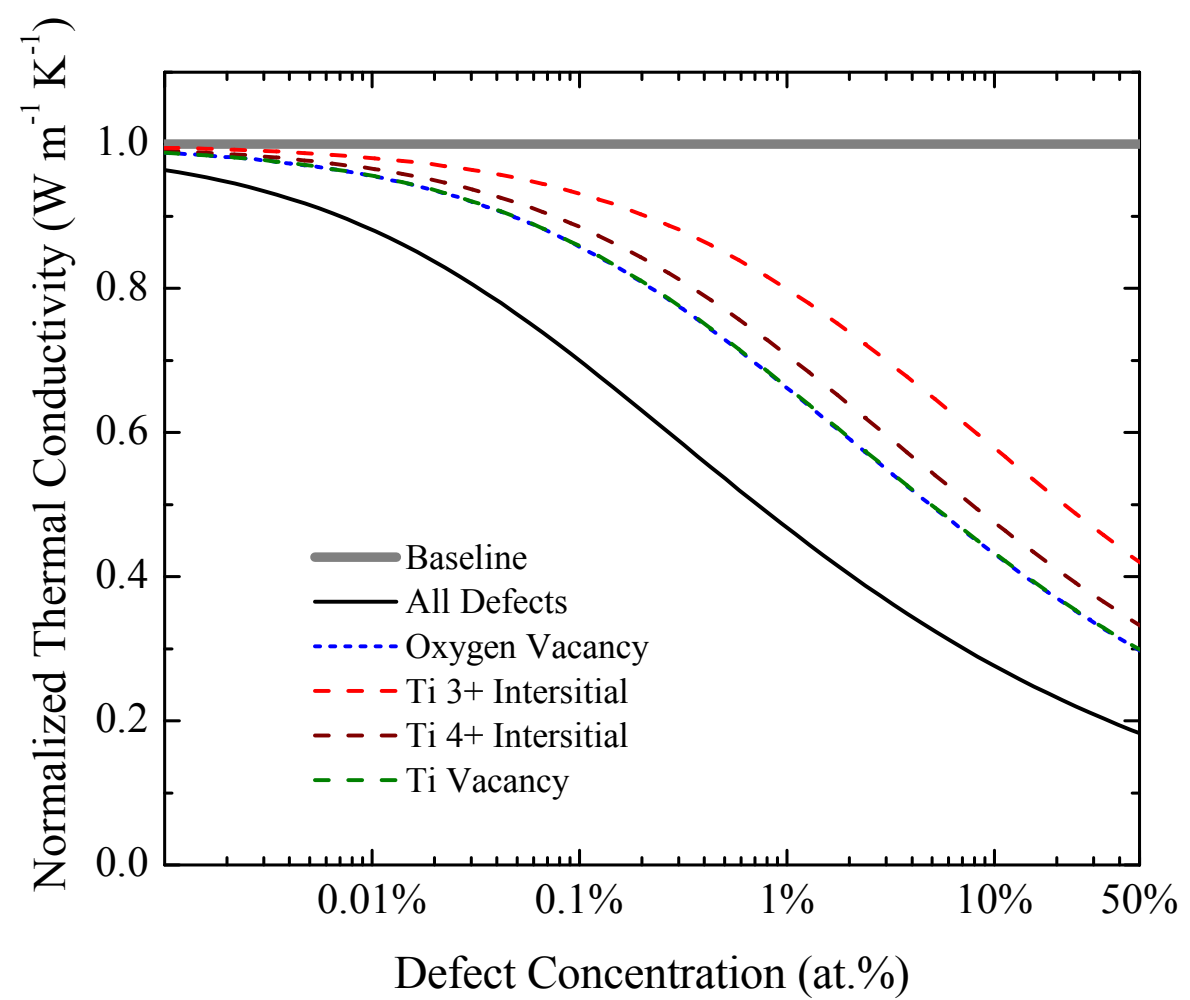

Figure 6.4: Normalized thermal conductivity with varying concentrations of titanium interstitials $\left(\mathrm{Ti}^{3+}\right.$ and $\left.\mathrm{Ti}^{4+}\right)$, titanium vacancies, and oxygen vacancies (as well as a simple linear summation of all four), determined by my analytical model for phonon thermal conductivity.

along with the baseline thermal scattering mechanisms, thus the scattering time for each type of defect is

$$
\begin{gathered}
\frac{1}{\tau}=\frac{1}{\tau_{P h}}+\frac{1}{\tau_{\text {Imp }}}+\frac{1}{\tau_{\text {Bound }}}+\frac{1}{\tau_{D O I}} \\
\frac{1}{\tau}=\frac{1}{\tau_{\text {Baseline }}}+\frac{1}{\tau_{D O I}}
\end{gathered}
$$

where $\tau_{D O I}$ is the scattering time for the defect of interest. This computation results in the thermal resistivities shown in Fig. 6.5, where we see that the major contribution to phonon scattering shift throughout the range of $\mathrm{pO}_{2}$. At high $\mathrm{pO}_{2}$ levels, the major defects impacting thermal transport are the oxygen and titanium vacancies. However, at very low $\mathrm{pO}_{2}$, the titanium interstitial concentration rises high enough to drive a significant decrease in the expected thermal resistivity. This has a profound implication in the process of dielectric breakdown, since it is the titanium interstitials that 
are the mobile defects under breakdown conditions, and this finding suggests that these defects do in fact significantly inhibit phonon transport at high concentrations.

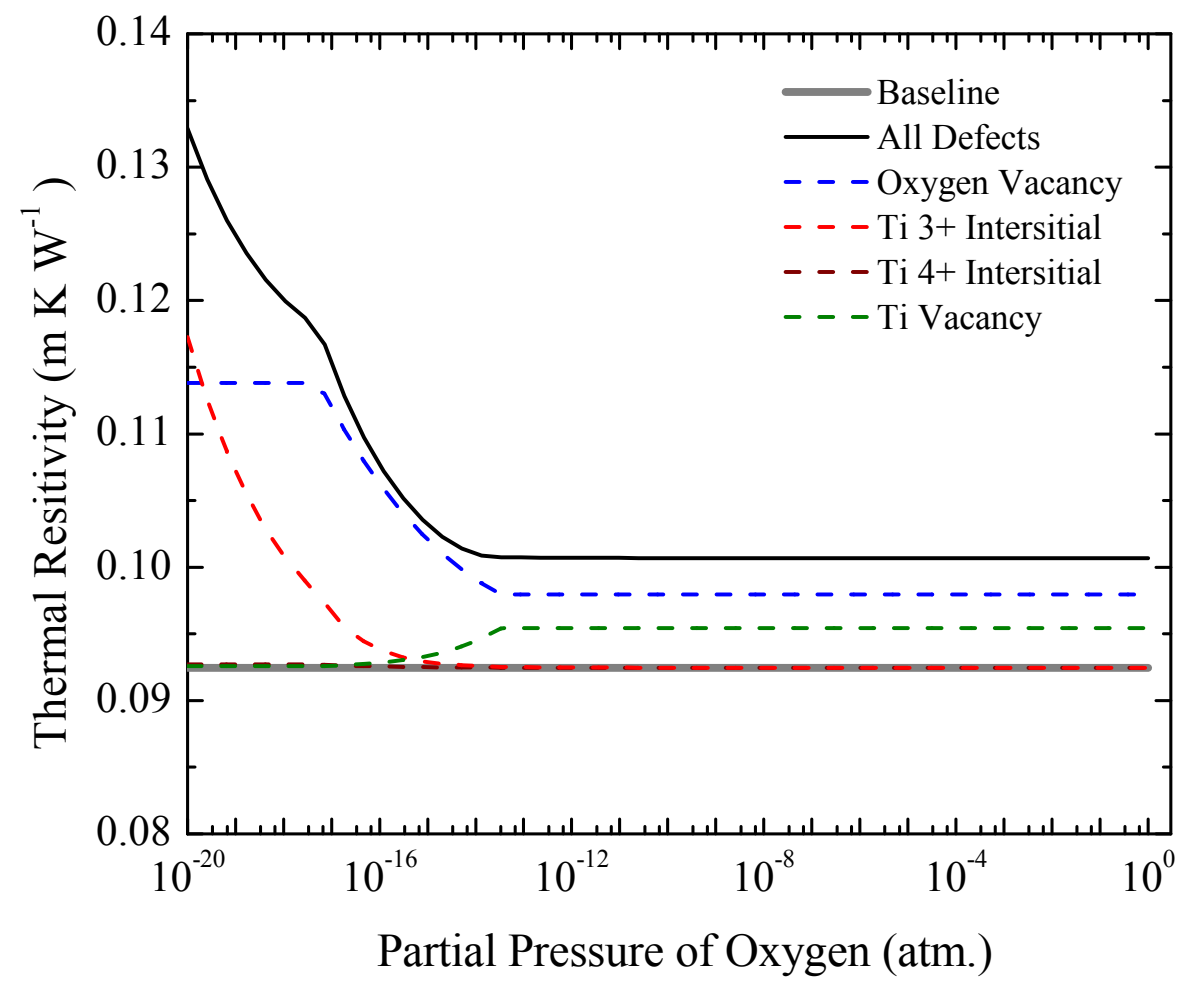

Figure 6.5: Thermal resistivity of rutile computed with contributions from equilibrium concentrations of known intrinsic defects along with the baseline thermal resistivity and the total thermal resistivity, which takes into account all of the intrinsic defects combined.

The lower valency cation interstitials $\left(\mathrm{Ti}^{4+}\right)$ play a negligible role in thermal transport as their concentrations are expected to be extremely low. At the transition into the reducing regime $\left(\sim 10^{-14}\right.$ atm.) the titanium vacancy concentration begins to decline and the contribution to the thermal resistivity becomes negligible by a $\mathrm{pO}_{2}$ of $\sim 10^{-16}$ atm. There is a distinct kink in the trend of the thermal resistivity with $\mathrm{pO}_{2}$ that corresponds to a change into extreme reducing environments expected at $5 \times 10^{-18} \mathrm{~atm}$. At this point the oxygen vacancy concentration is expected to level out and further reduction in the thermal conductivity comes from the increasing $\mathrm{Ti}^{3+}$ interstitial population.

\subsection{Impact of Defects on the Thermal Conductivity of Reduced $\mathrm{TiO}_{2}$}

The results of the model using the combination of equilibrium concentrations of defects, along with the measurements taken on crystals annealed at corresponding $\mathrm{pO}_{2}$ levels, are shown in Fig. 6.6. The 
thermal conductivity of the reduced rutile $\mathrm{TiO}_{2}$ decreases with decreasing partial pressure of oxygen. This effect does not come into play until the partial pressure of oxygen during the anneal is lower than $10^{-14} \mathrm{~atm}$. Total defect concentrations at partial pressures of oxygen below this level begin to exceed 0.7 at.\% as determined by the equilibrium concentrations of defects shown in Fig. 6.2. This defect concentration is into the regime where the thermal conductivity is significantly impacted and as the total concentration of defects is increased, the thermal conductivity drops compared to the baseline.

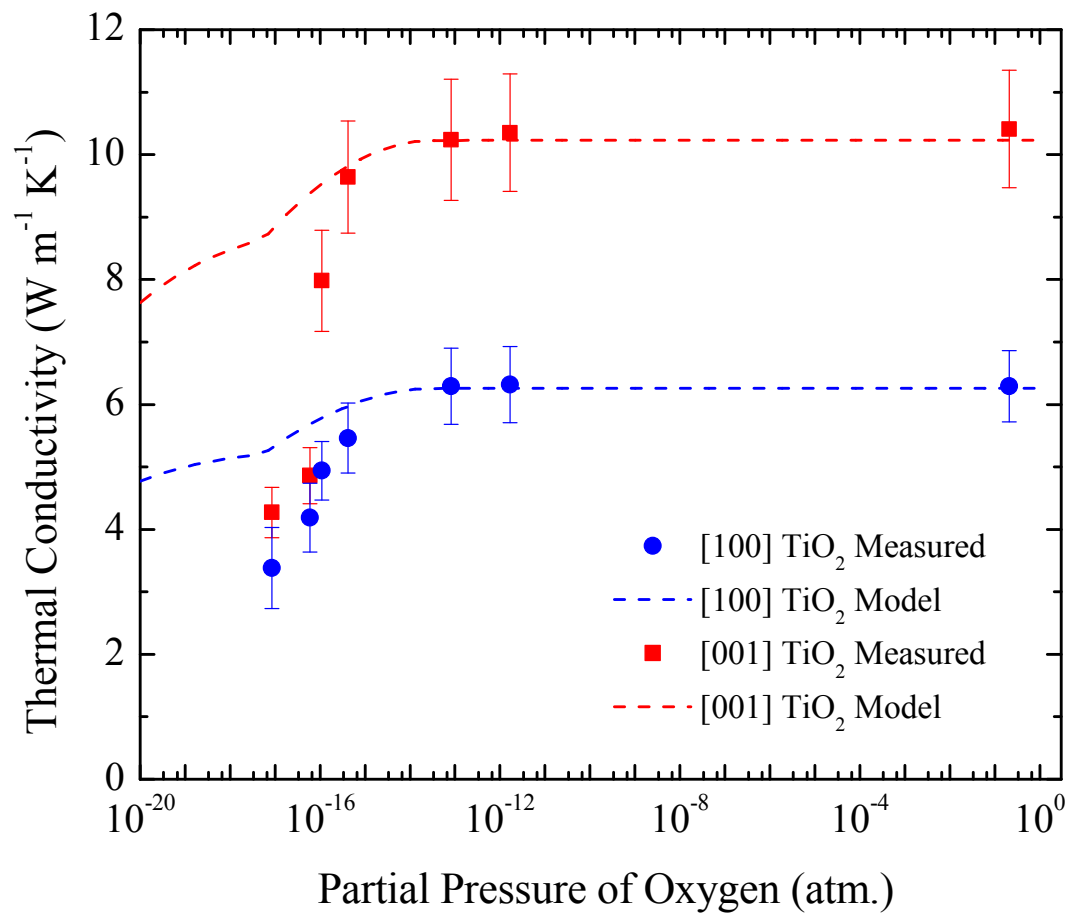

Figure 6.6: Thermal conductivity of [100]-oriented and [001]-oriented rutile $\mathrm{TiO}_{2}$ with various defect concentrations dictated by $\mathrm{pO}_{2}$ during anneals. The dotted lines indicate the modeled impact to thermal conductivity using defect concentrations found in Fig. 6.2.

This model is able to capture the onset of this decrease in the thermal conductivity as shown by the comparison to the data in Fig 6.6. At more extreme $\mathrm{pO}_{2}$ levels, the decrease in the measured thermal conductivity becomes more severe. With a total concentration of defects exceeding 1.5 at.\%, the system is sub-stoichiometric to a degree that we expect the formation of Magnéli phases. These low density, layered phases reduce the thermal conductivity well beyond the expected impact from non-interacting point defects. Furthermore, at the lowest $\mathrm{pO}_{2}$ levels that we could achieve, (as low as $8.4 \times 10^{-18} \mathrm{~atm}$.) the anisotropy in the thermal conductivity is no longer present. This suggests that 
the total thermal transport in the system is now being dominated by crystallites of an alternative phase of the reduced $\mathrm{TiO}_{2}$ and not the single crystal anisotropic rutile.

The expected threshold of sub-stoichiometry to induce a phase change into a Magnéli phase occurs at a O:Ti ratio of 1.97. Seen in Fig. 6.7, I have computed this ratio over the partial pressure of oxygen used to fabricate the samples in this study. I have included the expected $p \mathrm{O}_{2}$ thresholds considering all of the defects featured in the Brouwer diagram (Fig. 6.2) as well as considerations of only the oxygen and titanium-related defects. The calculation of this ratio is based off of the stoichiometric concentrations of each constituent atom modified by the concentrations of each defect as follows

$$
\mathrm{O}: \text { Ti ratio }=\frac{O\left(1-V_{O}\right)}{T i\left(1+T i_{i}^{3+}+T i_{i}^{4+}-V_{T i}\right)}
$$

where $O$ and $T i$ are the stoichiometric relative concentrations (2 and 1) and each other component is a concentration from the brouwer diagram.

From this analysis I would expect Magnéli phase formation to occur in the crystals that were annealed at $p \mathrm{O}_{2}$ levels below $6.4 \times 10^{-17}$ atm. If considering only the oxygen related defects, the $p \mathrm{O}_{2}$ threshold becomes somewhat lower $\left(2.1 \times 10^{-17} \mathrm{~atm}\right)$ and if only consider the titanium-related defects, the threshold is considerably higher $\left(1.7 \times 10^{-15} \mathrm{~atm}\right)$.

To investigate the change in phase that seems to occur at the lowest $p \mathrm{O}_{2}$ levels in our experiment, I turn to X-ray diffraction. The results of the X-ray diffraction scans shown in Fig. 6.8a show a distinct shift in peak location for the most severely reduced samples, corresponding to $p \mathrm{O}_{2}$ levels of $6.0 \times 10^{-17}$ and $8.4 \times 10^{-18}$. These samples are the two that show a total loss in anisotropy of the thermal conductivity. Additionally, these are the two samples that are below the threshold $p \mathrm{O}_{2}$ determined for Magnéli phase formation shown in Fig. 6.7. Since these samples are single crystals, the only $2 \theta$ peak that has strong intensity is the peak associated with the [002] direction (this particular set of X-ray diffraction scans was done on the c-axis oriented crystals). While this shift in the peak location does indicate a significant change in the crystal structure (change in the average lattice constant), the lack of any additional peaks stops us from making concrete conclusions that additional phases are forming. Figure $6.8 \mathrm{~b}$ shows the polycrystalline X-ray diffraction pattern taken from literature, comparing rutile to pure Magnéli phase powders [199]. From this its clear that the 


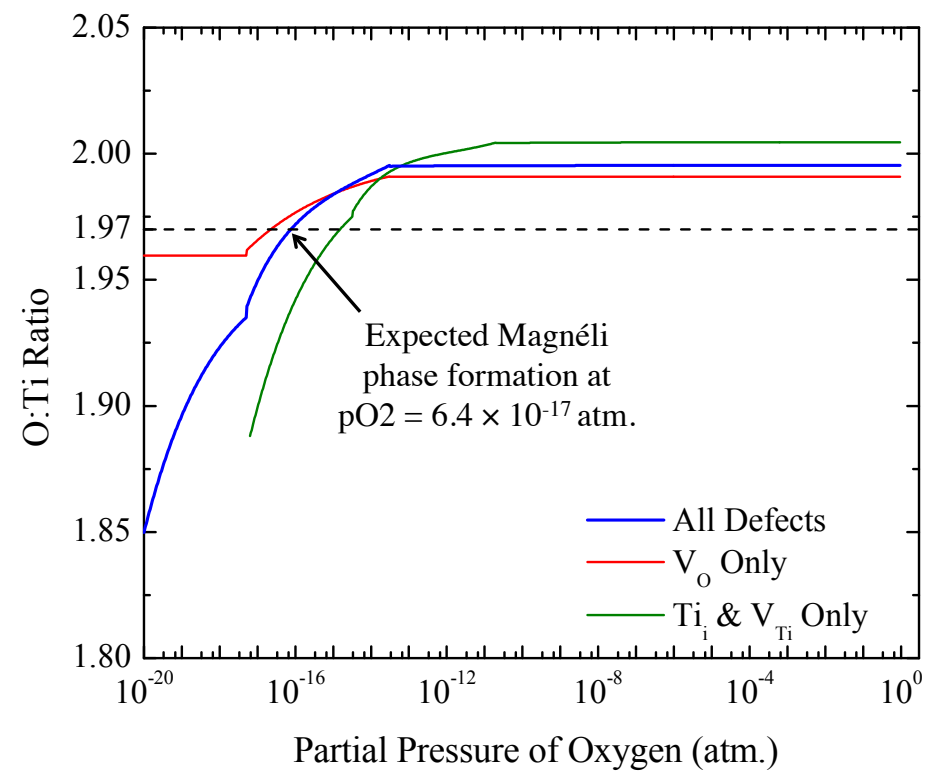

Figure 6.7: Ratio of oxygen to titanium in $\mathrm{TiO}_{2}$ dictated by the expected defect concentrations that arise from annealing at various partial pressures of oxygen. Included are the expected O:Ti ratios for all defects (blue) as well as just considering the oxygen (red) and titanium (green) related defects. The expected threshold for Magnéli phase formation occurs at a $\mathrm{O}$ :Ti ratio of 1.97 , corresponding to a $p \mathrm{O}_{2}$ level of $6.4 \times 10^{-17} \mathrm{~atm}$.

Magnéli phase featured has a peak that is shifted slightly higher in $2 \theta$ than the rutile [002] peak, indicating that the two samples at the lowest $p \mathrm{O}_{2}$ have started going through the phase transition.

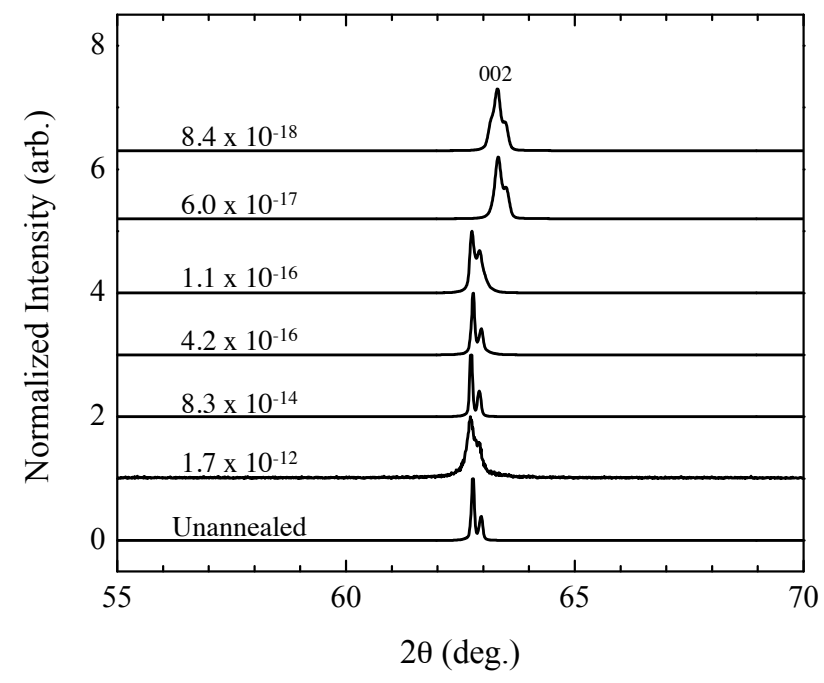

(a) X-ray diffraction scans of reduced $\mathrm{TiO}_{2}$

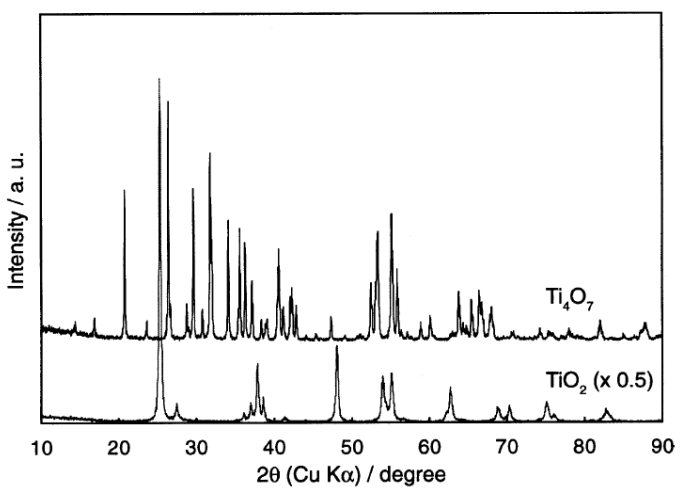

(b) X-ray diffraction pattern of $\mathrm{Ti}_{4} \mathrm{O}_{7}$

Figure 6.8: (a) X-ray diffraction data taken on all of the reduced $\mathrm{TiO}_{2}$ crystals used in this study and (b) literature values for diffraction scans from rutile and Magnéli phase powders [199].

With help from colleagues in Professor Dickey's group at North Carolina State University, we obtained electron diffraction patterns, which allow us to see additional crystallographic peaks around 


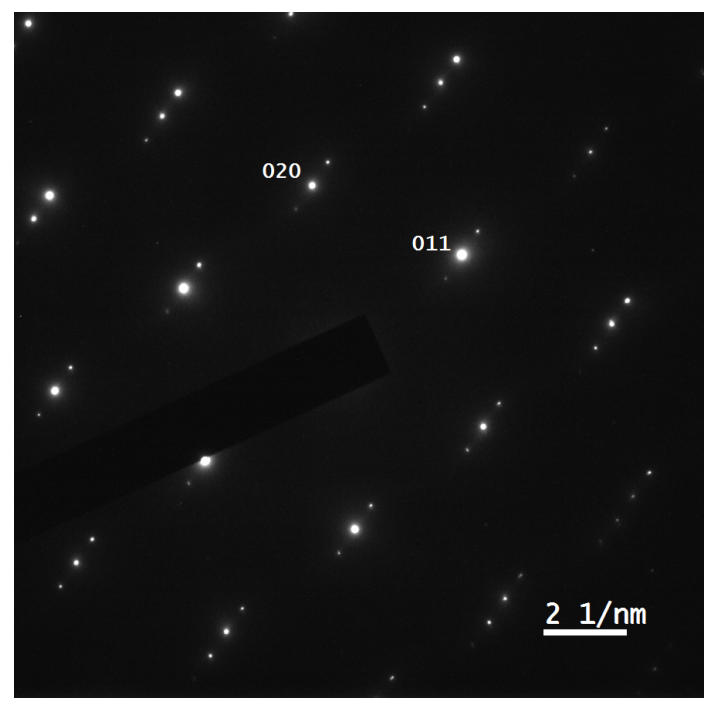

Figure 6.9: Electron diffraction patterns of the defected region of the $\mathrm{Ti}_{n} \mathrm{O}_{2 n-1}$ crystal that has been annealed at a $\mathrm{pO}_{2}$ level of $8.4 \times 10^{-18}$ atm. Taken along the [100] zone axis with a JEOL $2010 \mathrm{~F}$ at $200 \mathrm{kV}$.

the $\mathrm{TiO}_{2}$ peaks in the degraded crystal. Figure 6.9 shows the electron diffraction pattern of the rutile in the [100] direction, the primary intensity peaks are clearly defined and labeled. There is also a concrete signature of a layered secondary phase in the diffraction pattern in the form of smaller peaks around the primary peaks. This diffraction signature mimics that seen in other studies on Magnéli phase materials indicative of a superlattice structure aligned along the (075) direction with spacing between layers of $1.4 \mathrm{~nm}$. Additional diffraction patterns taken from this sample vary in the extent of this defect signature. Figure 6.10 shows two other diffraction scans that show very little signature of secondary phases or the signature of a highly defective crystal that has yet to form secondary phases. Since the spatial window of these images is on the order of magnitude of 100's $\mathrm{nm}$, our measurement (covering 10's $\mu \mathrm{m}$ ) would incorporate many of these regions, resulting in a spatial average that includes Magnéli phases as well as defected rutile.

We are able to identify the onset of the layered defects that lead to the new ordered phase with TEM analysis of a crystal that has been annealed at a $\mathrm{pO}_{2}$ level of $4.2 \times 10^{-16} \mathrm{~atm}$, which is the highest $\mathrm{pO}_{2}$ for which we see a reduction in the thermal conductivity from defects. Figure 6.11a shows the formation of extended defects from the high concentration of point defects and Fig. 6.11b shows the corresponding electron diffraction pattern for this region, with peak widening in the crystallographic directions that correspond to the extended defects. This bright field, phase contrast imaging allows us to directly see the crystallographic shear planes that form by the ordering of the stacking faults as 

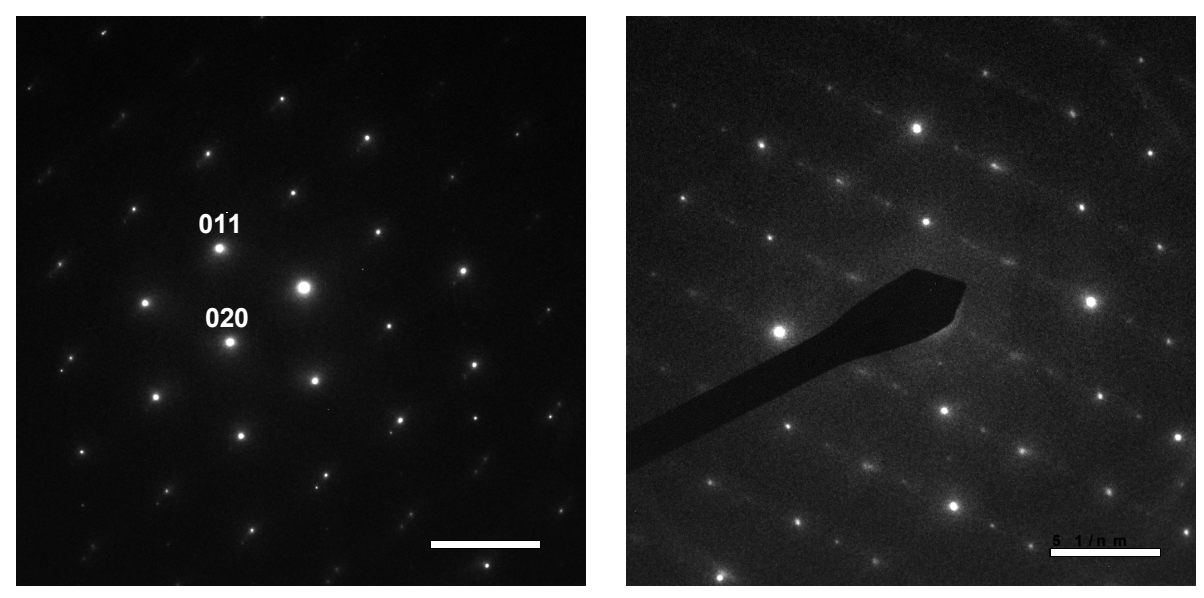

Figure 6.10: Electron diffraction patterns of other regions of the $\mathrm{Ti}_{n} \mathrm{O}_{2 n-1}$ crystal that has been annealed at a pO level of $8.4 \times 10^{-18} \mathrm{~atm}$ demonstrating the variety in extent of defect and secondary phase concentration. These were taken along the [100] with a JEOL 2000FX at $200 \mathrm{kV}$.

the titanium interstitials rearrange, discussed in Sect. 2.4 as growing along 132 planes. This growth direction corresponds to the smearing of the diffraction peaks seen in Fig. 6.11b which is accentuated in the highly defected region that has yet to form an ordered phase in Fig. 6.10. The TEM analysis from this additional sample gives an indication that, while we have not yet formed full Magnéli phases, the defect concentration is high enough that the point defects are beginning to interact even though the model that I have projected (based on non-interacting defects) still captures the data.

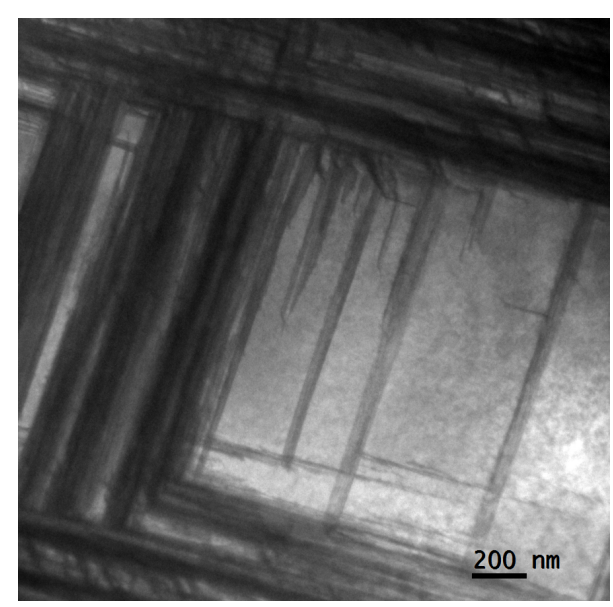

(a) Phase contrast TEM

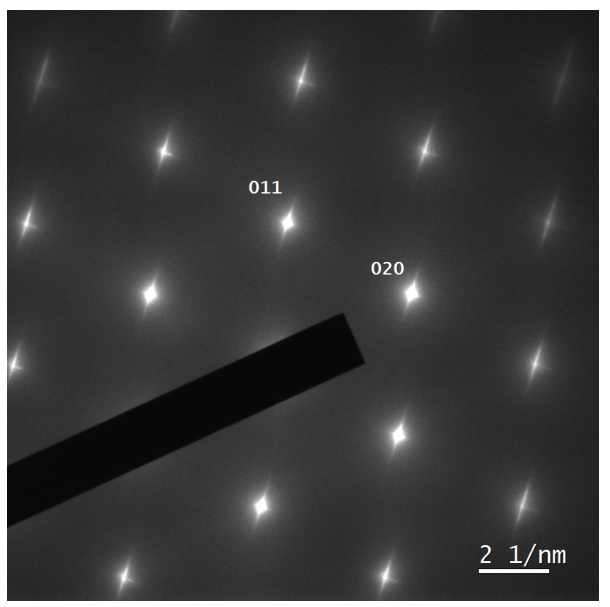

(b) Electron diffraction pattern

Figure 6.11: TEM analysis of a $\mathrm{TiO}_{2}$ crystal annealed at a $\mathrm{pO}_{2}$ level of $4.6 \times 10^{-16}$ atm. (a) Low resolution phase contrast image shows the presence of extended defects and (b) electron diffraction patterns show peak widening in the crystallographic directions that correspond to the extended defects. 
In conclusion, there is clear evidence of the interaction of intrinsic point defects with phonons in rutile $\mathrm{TiO}_{2}$. Control over the intrinsic point defect profile was accomplished using high temperature anneals and regulated partial pressures of oxygen. At low partial pressures of oxygen, the total intrinsic defect concentration is driven well beyond 1 at.\% resulting in a significant reduction in the thermal conductivity.

As the sub-stoichiometry becomes more severe, the rutile $\mathrm{TiO}_{2}$ undergoes a phase transition into a less dense, layered Magnéli phase. This phase transition results in a reduction in the thermal conductivity that is well beyond the expected reduction from a homogeneous, non-interacting profile of point defects.

Using an analytical model for the thermal conductivity, I have shown that the various contributions to phonon scattering by different types of intrinsic defects varies with different regimes of reduction in $\mathrm{TiO}_{2}$. The model indicates that the oxygen and titanium vacancies play the most significant role in intrinsic point defect scattering at high $\mathrm{pO}_{2}$, but at low $\mathrm{pO}_{2}$, the titanium interstitials $\left(\mathrm{Ti}^{3+}\right)$ begin to dominate the phonon scattering. These findings have significance particularly to the application of dielectric breakdown, showing the role of the various defects that drift and concentrate during prolonged application of high electric fields in dielectric crystals. 
Opportunity for Further Investigation: Driving a phase change into Magnéli phases of $\mathrm{TiO}_{2}$ using single crystals is not the common technique used in literature $[178,185,190,202]$; the initial $\mathrm{TiO}_{2}$ is usually polycrystalline. This offers a unique opportunity to further study the nature of the change in phase in a controlled way between defected rutile and a Magnéli phase $\operatorname{Ti}_{n} \mathrm{O}_{2 n-1}$ using the single crystals that were reduced in this study. Our initial investigation into this phase change offers concrete evidence that a change to a highly defected new phase is occurring, which is sufficient to understand the trend in the thermal data, but there are some interesting features that could be explored further. It seems from the TEM analysis that the formation of these phases is nucleating from within the crystal, while the near-surface region is less defected (near-surface in this case is relative to the dimension of the TEM lamella, the thermal probe used is deep enough to fully sample the defective region, as seen in the thermal conductivity data). Additionally, the shift in the X-ray diffraction peak would indicate that the lattice constant in the [001] direction is getting smaller, which seems to be counterintuitive as these phases are less dense than rutile. High resolution microscopy (TEM or STEM) could lend significant insight into these dynamics and result in an entirely new set of publishable findings on the nature of the phase transition from rutile to Magnéli phases. 


\section{$7 \quad$ Summary}

Advances in technology will continue to push constituent materials, such as functional oxides, to extreme environments, dimensionalities, and configurations. Understanding the interaction of thermal carriers with the defects that arise from these conditions is a major component in the materials design process that enables progress. With advances in processing, complex defect profiles in functional oxides can lead to new scattering mechanisms affecting thermal transport, with thermal conductivity dictated by defects ranging from boundaries to low concentration impurities. Under extreme operation, defect migration can also lead to altered profiles that induce a variety of thermal transport regimes. This work serves to address these material configurations and fully characterize the resulting impact on thermal transport at the nanoscale. Thermal measurements accompanied by modeling for three different material systems have been presented to illustrate the general interaction of phonons with defects in functional oxides.

The motivation behind studying thermal transport in functional oxides stems from a number of applications. In microelectronics applications, functional oxides are generally preferred to have higher thermal conductivities in order to dissipate built up heat. In applications such as thermal barrier coatings and thermoelectrics, desirable properties include very low thermal conductivities to minimize heat flux through the material. In some advanced applications, continuous tuning of the thermal conductivity is of interest and leads to some very interesting material design challenges.

In each regime of control of the thermal conductivity, understanding thermal transport and the interaction of phonons with defects is crucial. I outline the nature and analytical form of phonon transport in Sections $2.1 \& 2.2$. I discuss not only the method for modeling phonon transport, but also review the form of various defect scattering mechanisms including grain boundaries and point defects. The formation of point defects in oxides is crucial to the defect profiles present in these materials, as well as determining the processing conditions necessary to mimic exposure to extreme environments. I explain the thermodynamics behind the determination of defect equilibrium concentrations in Sect. 2.3. In Sect. 2.5, I address the major application that drives the final study in my dissertation which is dielectric breakdown. A key aspect of each of these studies is the accurate measurement of the thermal conductivity of these materials. I accomplish this using time domain 
thermoreflectance and describe the measurement and analysis technique in depth in Ch. 3.

I have shown experimentally that functional oxide defects can be used to control the spectrum of heat carrying phonons present in these materials. In Ch. 4, this is done using grain boundaries in $\mathrm{BaTiO}_{3}$. The thermal conductivity increases with increasing grain size in $\mathrm{BaTiO}_{3}$ thin films, up to a film boundary limited thermal conductivity. This result demonstrates the spectral nature of phonons in functional oxides and highlights the presence of size effects dictated by grain size. Boundary scattering is particularly pertinent in $\mathrm{BaTiO}_{3}$ as it is a primary constituent in multilayer ceramic capacitors; an application which requires thermal stability and is consistently being driven to smaller size scales. Also dictated by processing, extrinsic point defects can impact the thermal conductivity in complex ways through multiple defect interactions.

Shown in Ch. 5, the thermal conductivity of Dy doped CdO films can be increased and decreased both by electronic and phonon thermal conductivity using variations in Dy doping. As dopant concentration is increased initially, the substitutional Dy shifts the equilibrium concentration of oxygen vacancies down, resulting in less overall phonon scattering. Continuous increase in Dy defect concentrations leads to an overall decrease in the phonon scattering rate eventually, but this is also coupled with an increasing electronic population which results in a dual peak signature, the magnitude of which was manipulated by reducing the sample temperature to $80 \mathrm{~K}$. In this chapter, I show that the model for phonon-defect scattering can be used to gain additional information about the defect interactions through a comparison to the measured thermal conductivity. This simple analytical model indicates an interaction, supported by the literature, where high enough concentrations of Dy result in compensation by cation vacancies, significantly increasing the phonon scattering rate in the secondary regime shown in Fig. 5.5.

The intrinsic defect profile can also lead to interesting interactions between defects that dictate phonon scattering rates. I show the effect of increasing the intrinsic defect concentration on the thermal conductivity of $\mathrm{TiO}_{2}$ in $\mathrm{Ch}$. 6. In this case, the thermal conductivity is reduced after the introduction of a significant concentration of defects via low oxygen partial pressure annealing. The reduction in the thermal conductivity is accelerated by the formation of secondary layered phases at severe $\mathrm{Ti}_{n} \mathrm{O}_{2-n}$ sub-stoichiometry. The expected contribution of each type of defect can be identified using the analytical model for the thermal conductivity, and demonstrates a dominant scattering mechanism of oxygen vacancies at higher partial pressure of oxygen and titanium interstitials at very 
low partial pressure of oxygen. The results of this study will be critical in identifying the role that thermal transport plays in the dielectric breakdown process, especially under high enough electronic fields where significant defect migration takes place.

These three studies cover various aspects of defects that arise from processing and application of functional oxide materials. Along with the background presented in this dissertation, these conclusions frame a concrete understanding of the interaction of phonons with defects in functional oxide materials. The various fields that deal with functional oxide thermal engineering will be benefitted by the insights gained from these studies.

\subsection{Opportunities for Further Investigation}

Throughout this dissertation I have outlined a number of opportunities for further investigation relating to the study of thermal transport and thermal characterization. Along with these opportunities, which I have summarized below, there are direct extensions of each project outlined in Ch. 4, 5, and 6.

An extension of the $\mathrm{BaTiO}_{3}$ work is in the thermal boundary conductance across nano-grained boundaries with low thermal conductivity constituents. There is an effort in the field of solid-liquid thermal boundary conductance research to accurately determine this value experimentally. These material systems offer an interesting opportunity to test analysis techniques that are sensitive to boundaries adjacent to low thermal conductivity materials. If accurate analysis is possible, with the data that I have already taken, the understanding of spectral phonon scattering dictated by grain boundaries can be extended to a study of a limited phonon spectrum on one side of an interface that is controlled in the long wavelength limit by grain size.

The $\mathrm{CdO}$ work can be extended in two different ways. In terms of thermal measurements, modeling of the changes in electron transport could help to establish a full picture of the trends in the thermal conductivity without having to decompose the data before comparison to the model. In terms of the material system, the control over the growth and defect states of this system offer some interesting extensions. One which is being explored is the control of dislocation density in thin $\mathrm{CdO}$ films. Our collaborators at North Carolina State University are working on modulating dislocation density at the CdO-substrate interface, after which we may be able to measure thermal boundary conductance changes and study the effect of dislocations on thermal transport across an interface. 
The $\mathrm{TiO}_{2}$ study can be extended into direct application and in-stiu testing of dielectric breakdown. Now that there is clear evidence that these point defect concentrations in $\mathrm{TiO}_{2}$ significantly inhibit phonon transport, we can begin to test crystals which have been electrically degraded and attempt to resolve the known defected layer that results from defect migration, an experiment that is ideal for TDTR. If that experimental resolution is possible, the next step will be use the TDTR transducer as an electrode and monitor the change in the thermal conductance at or near the interface as point defects migrate towards, and build up at the electrode.

I will continue to work with Prof. Hopkins and the other collaborators that we have for each one of these projects over the next few years to develop these extensions. Additionally, I will help Prof. Hopkins pursue a number of the other opportunities for further research that have been discussed in this dissertation and are summarized below.

Pulsed-CW FDTR: The FDTR configuration featured in Fig. 3.1 is one that has been specifically built for some interesting potential experiments. Utilizing the pulsed-pump with the CW probe, it is theoretically possible to reconstruct the time domain signal of the pulsed heating event by analyzing the pulse frequency component of the CW probe. With a large enough frequency bandwidth on a lock-in detector along with a boxcar averaging system that can recreate the pulse window, one should be able to resolve the thermoreflectance decay between pulses.

Low thermal conductivity thermal boundary conductance: The sensitivity to the thermal boundary conductance adjacent to low thermal conductivity materials is generally low. For a boundary in series with a low thermal conductivity layer, the decay of the thermoreflectance will be dominated by the thermal conductivity of the layer and small changes in the thermal boundary conductance will result in small changes in the overall signal characteristics. This issue is often overlooked in measurements of thermal boundary conductance adjacent to thermally resistive materials such as solid-liquid thermal boundary conductance. There is an opportunity here to address this issue comprehensively and also use in depth analysis of measurement sensitivity to identify systems that can accurately be measured using multi-layer thermal diffusion techniques such as TDTR. Precise measurement can only be achieved in systems with very low thermal boundary conductances, in the case of liquids this would be a solid-liquid interface with a low work of adhesion 
such as a hydrophobic solid and water or an oleophobic solid and oil.

Analytical model for grain boundary scattering: A simple model for grain boundary scattering has captured the trends in the data relatively well. While this fit and theoretical form of phonon-boundary scattering leads to an accurate and efficient representation of the physical system, there is always room for improvement of models. Measurement of the thermal conductivity of nano-grained $\mathrm{BaTiO}_{3}$ is an experiment where there may be an interesting investigation into the physical form of the phonon-grain boundary interaction. For instance, extension of the grain boundary scattering time to include a phonon wavelength dependence, as it is treated in some recent works in literature [286, 287], may yield some additional insight beyond the trends and confirmation of spectral scattering seen in this experiment.

Grain size directed thermal rectification: Thermal rectification, or the bias of heat flow in a forward direction, can be achieved by graded size effects. Using chemical solution deposition and controlled grain size, one could theoretically accomplish cross-plane thermal rectification by incrementally layering $\mathrm{BaTiO}_{3}$ films of larger and larger grain size. This would establish a thermal conductivity gradient, forcing a significant difference in heat flux from the top of the film down as compared the the bottom of the film up. This effect could be directly measured with TDTR using a Pt-Sapphire substrate and measuring transport on opposite sides of the thermal rectifying film.

Exploration of Magnèli phase formation in single crystal rutile: Driving a phase change into Magnèli phases of $\mathrm{TiO}_{2}$ using single crystals is not the common technique used in literature $[178,185,190,202]$; the initial $\mathrm{TiO}_{2}$ is usually polycrystalline. This offers a unique opportunity to further study the nature of the change in phase in a controlled way between defected rutile and a Magnèli phase $\mathrm{Ti}_{n} \mathrm{O}_{2 n-1}$ using the single crystals that were reduced in this study. Our initial investigation into this phase change offers concrete evidence that a change to a highly defected new phase is occurring, which is sufficient to understand the trend in the thermal data, but there are some interesting features that could be explored further. It seems from the TEM analysis that the formation of these phases is nucleating from within the crystal, while the near-surface region is less defected (near-surface in this case is relative to the dimension of the TEM lamella, the thermal 
probe used is deep enough to fully sample the defective region, as seen in the thermal conductivity data). Additionally, the shift in the X-ray diffraction peak would indicate that the lattice constant in the [001] direction is getting smaller, which seems to be counterintuitive as these phases are less dense than rutile. High resolution microscopy (TEM or STEM) could lend significant insight into these dynamics and result in an entirely new set of publishable findings on the nature of the phase transition from rutile to Magnèli phases.

\subsection{Contributions to the Scientific Body of Knowledge}

The findings of the work presented in this dissertation will help to guide the path of thermal engineering in functional oxide materials in the future. Understanding the interplay of various defect mechanisms and the major thermal carriers in these materials will enable advances in applications ranging from energy generation to microelectronics. Furthermore, this experimental work serves as validation of the fundamental physics involved and helps to elucidate the nature of phonons in functional oxides.

With regards to impact within the field of nanoscale thermal characterization, the steps taken to obtain these measurements will serve as a guide to future thermal characterization of functional oxides. The experimental setup, quality of sample fabrication, and additional characterization done in these studies is exemplary in terms of achieving impactful and publishable results. Lastly, the work that I have done in setting up facilities including sample treatment and preparation, temperature variable testing, electrically variable testing, pulsed-CW FDTR, and TDTR in the Exsite Lab at UVA has had a significant impact on the productivity and diversity of projects that have taken place during my graduate studies and will continue to have an impact after I have graduated. 


\subsection{Published Work}

The work discussed in Ch. $4 \& 5$ has been published in the form of three manuscripts, two in Applied Physics Letters and one in Nature Materials. I am the first author on two of the three manuscripts. The work in Ch. 6 is currently in the process of being submitted as well. In addition to these manuscripts, I have been involved with additional published findings, all of which are related to nanoscale thermal transport. Some of the manuscripts listed here have directly impacted the design of the discussed projects.

15. B. F. Donovan, D. M. Long, A. Moballegh, E. C. Dickey, and P. E. Hopkins, "Impact of intrinsic point defect concentration on thermal transport in $\mathrm{TiO}_{2}, "$ In Preparation, 2016

14. B. F. Donovan, W. A. Jensen, N. Liu, J. A. Floro, and P. E. Hopkins, "Strain induced reduction of silicon thermal conductivity with aluminum inclusions," In Preparation, 2016

13. L. Chen, B. F. Donovan, P. E. Hopkins, and S. J. Poon, "Length scale dependence of the thermal conductivity accumulation in nanograined Si-Ge alloys," In Preparation, 2016

12. B. F. Donovan, C. J. Szwejkowski, A. Giri, R. Cheaito, J. T. Gaskins, and P. E. Hopkins, "Contrast of macro-scale wetting and nano-scale thermal interactions between solids and fluorinated liquids," In Preparation, 2016

11. B. F. Donovan, A. Giri, J. T. Gaskins, and P. E. Hopkins, "Localized thin film dewetting and sourced and monitored via ultra-fast optics," Under Review, 2016

10. L. D. Zarzar, B. S. Swartzentruber, B. F. Donovan, P. E. Hopkins, and B. Kaehr, "Using laser-induced thermal voxels to pattern diverse materials at the solid-liquid interface," Under Review, 2016

9. A. Giri, J.-P. Niemelä, T. Tynell, J. T. Gaskins, B. F. Donovan, M. Karppinen, and P. E. Hopkins, "Heat-transport mechanisms in molecular building blocks of inorganic/organic hybrid superlattices," Phys. Rev. B, vol. 93, p. 115310,112016

8. B. F. Donovan, E. Sachet, J.-P. Maria, and P. E. Hopkins, "Interplay between mass-impurity and vacancy phonon scattering effects on the thermal conductivity of doped cadmium oxide," Applied Physics Letters, vol. 108, no. 2, 021901, 2016

7. E. Sachet, C. T. Shelton, J. S. Harris, B. E. Gaddy, D. L. Irving, S. Curtarolo, B. F. Donovan, P. E. Hopkins, P. A. Sharma, A. L. Sharma, J. Ihlefeld, S. Franzen, and J.-P. Maria, "Dysprosium-doped cadmium oxide as a gateway material for mid-infrared plasmonics," Nature Materials, vol. 14, no. 4, pp. 414-420, Apr. 2015 
6. J. Loureiro, T. Mateus, S. Filonovich, M. Ferreira, J. Figueira, A. Rodrigues, B. F. Donovan, P. Hopkins, and I. Ferreira, "Hydrogenated nanocrystalline silicon thin films with promising thermoelectric properties," Applied Physics A, pp. 1-6, 2015

5. R. Cheaito, J. T. Gaskins, M. E. Caplan, B. F. Donovan, B. M. Foley, A. Giri, J. C. Duda, C. J. Szwejkowski, C. Constantin, H. J. Brown-Shaklee, J. F. Ihlefeld, and P. E. Hopkins, "Thermal boundary conductance accumulation and interfacial phonon transmission: measurements and theory," Phys. Rev. B, vol. 91, p. 035432,32015

4. C. J. Szwejkowski, N. C. Creange, K. Sun, A. Giri, B. F. Donovan, C. Constantin, and P. E. Hopkins, "Size effects in the thermal conductivity of gallium oxide $\left(\beta-\mathrm{G}_{2} 2 \mathrm{O}_{3}\right)$ films grown via open-atmosphere annealing of gallium nitride," Journal of Applied Physics, vol. 117, no. 8, 084308, p. 084 308, 2015

3. A. Giri, J. T. Gaskins, B. F. Donovan, C. Szwejkowski, R. J. Warzoha, M. A. Rodriguez, J. Ihlefeld, and P. E. Hopkins, "Mechanisms of nonequilibrium electron-phonon coupling and thermal conductance at interfaces," Journal of Applied Physics, vol. 117, no. 10, 105105, p. 105 105, 2015

2. B. F. Donovan, C. J. Szwejkowski, J. C. Duda, R. Cheaito, J. T. Gaskins, C.-Y. Peter Yang, C. Constantin, R. E. Jones, and P. E. Hopkins, "Thermal boundary conductance across metal-gallium nitride interfaces from 80 to 450 K," Applied Physics Letters, vol. 105, no. 20, 203502, p. 203502,2014

1. B. F. Donovan, B. M. Foley, J. F. Ihlefeld, J.-P. Maria, and P. E. Hopkins, "Spectral phonon scattering effects on the thermal conductivity of nano-grained barium titanate," Applied Physics Letters, vol. 105, no. 8, 082907, p. 082907,2014 


\section{References}

[1] D. G. Cahill, W. K. Ford, K. E. Goodson, G. D. Mahan, A. Majumdar, H. J. Maris, R. Merlin, and S. R. Phillpot, "Nanoscale thermal transport," Journal of Applied Physics, vol. 93, no. 2, pp. 793-818, 2003.

[2] D. G. Cahill, P. V. Braun, G. Chen, D. R. Clarke, S. Fan, K. E. Goodson, P. Keblinski, W. P. King, G. D. Mahan, A. Majumdar, H. J. Maris, S. R. Phillpot, E. Pop, and L. Shi, "Nanoscale thermal transport. II. 2003-2012," Applied Physics Reviews, vol. 1, no. 1, pp. $1-45,2014$.

[3] E. Pop, S. Sinha, and K. E. Goodson, "Heat generation and transport in nanometer-scale transistors," Proceedings of the IEEE, vol. 94, no. 8, pp. 1587-1601, 2006.

[4] A. M. Marconnet, M. Asheghi, and K. E. Goodson, "From the casimir limit to phononic crystals: 20 years of phonon transport studies using silicon-on-insulator technology," Journal of Heat Transfer, vol. 135, no. 6, p. 061601, May 2013.

[5] A. von Hippel, R. G. Breckenridge, F. G. Chesley, and L. Tisza, "High dielectric constant ceramics," Industrial and Engineering Chemistry, vol. 38, no. 11, pp. 1097-1109, 1946.

[6] G. Arlt, D. Hennings, and G. de With, "Dielectric properties of fine-grained barium titanate ceramics," Journal of Applied Physics, vol. 58, no. 4, pp. 1619-1625, 1985.

[7] J. Ho, T. Jow, and S. Boggs, "Historical introduction to capacitor technology," Electrical Insulation Magazine, IEEE, vol. 26, no. 1, pp. 20-25, 2010.

[8] M.-J. Pan and C. A. Randall, "A brief introduction to ceramic capacitors," Electrical Insulation Magazine, IEEE, vol. 26, no. 3, pp. 44-50, 2010.

[9] S. Trolier-McKinstry and P. Muralt, "Thin film piezoelectrics for MEMS," Journal of Electroceramics, vol. 12, no. 1, pp. $7-17,2004$.

[10] M. D. Maeder, D Damjanovic, and N Setter, "Lead free piezoelectric materials," Journal of Electroceramics, vol. 13, no. 1-3, pp. 385-392, 2004.

[11] S. R. Anton and H. A. Sodano, "A review of power harvesting using piezoelectric materials (2003-2006)," Smart materials and Structures, vol. 16, no. 3, R1, 2007.

[12] C. J. Barbé, F. Arendse, P. Comte, M. Jirousek, F. Lenzmann, V. Shklover, and M. Grätzel, "Nanocrystalline titanium oxide electrodes for photovoltaic applications," Journal of the American Ceramic Society, vol. 80, no. 12, pp. 3157-3171, 1997.

[13] M. Grätzel, "Solar energy conversion by dye-sensitized photovoltaic cells," Inorganic chemistry, vol. 44, no. 20, pp. 6841$6851,2005$.

[14] P. Ravirajan, A. M. Peiró, M. K. Nazeeruddin, M. Graetzel, D. D. C. Bradley, J. R. Durrant, and J. Nelson, "Hybrid polymer/zinc oxide photovoltaic devices with vertically oriented zno nanorods and an amphiphilic molecular interface layer," The Journal of Physical Chemistry B, vol. 110, no. 15, pp. 7635-7639, 2006.

[15] W. R. Thurber and A. J. H. Mante, "Thermal conductivity and thermoelectric power of rutile ( $\left.\mathrm{TiO}_{2}\right)$," Phys. Rev., vol. 139, A1655-A1665, 5A 1965.

[16] G. Mahan, "Good thermoelectrics," in, ser. Solid State Physics, vol. 51, Academic Press, 1997, pp. 81 -157.

[17] M. K. Nowotny, T. Bak, and J. Nowotny, "Electrical properties and defect chemistry of $\mathrm{TiO}_{2}$ single crystal. II. thermoelectric power," The Journal of Physical Chemistry B, vol. 110, no. 33, pp. 16 283-16 291, 2006, PMID: 16913754.

[18] G. J. Snyder and E. S. Toberer, "Complex thermoelectric materials," Nat Mater, vol. 7, no. 2, pp. 105-114, Feb. 2008.

[19] M. Zebarjadi, K. Esfarjani, M. S. Dresselhaus, Z. F. Ren, and G. Chen, "Perspectives on thermoelectrics: from fundamentals to device applications," Energy Environ. Sci., vol. 5, pp. 5147-5162, 12012. 
[20] S. Ogale, T. Venkatesan, and M. Blamire, Functional Metal Oxides: New Science and Novel Applications. Wiley, 2013.

[21] C. Kittel and P. McEuen, Introduction to solid state physics. Wiley New York, 1986, vol. 8.

[22] G. Srivastava, The Physics of Phonons. Taylor \& Francis, 1990.

[23] G. Chen, Nanoscale Energy Transport and Conversion : A Parallel Treatment of Electrons, Molecules, Phonons, and Photons: A Parallel Treatment of Electrons, Molecules, Phonons, and Photons. Oxford University Press, USA, 2005.

[24] D. G. Cahill, "Analysis of heat flow in layered structures for time-domain thermoreflectance," Review of Scientific Instruments, vol. 75, no. 12, pp. 5119-5122, 2004.

[25] A. J. Schmidt, X. Chen, and G. Chen, "Pulse accumulation, radial heat conduction, and anisotropic thermal conductivity in pump-probe transient thermoreflectance," Review of Scientific Instruments, vol. 79, no. 11, 114902, p. $114902,2008$.

[26] P. E. Hopkins, J. R. Serrano, L. M. Phinney, S. P. Kearney, T. W. Grasser, and C. T. Harris, "Criteria for cross-plane dominated thermal transport in multilayer thin film systems during modulated laser heating," Journal of Heat Transfer, vol. 132, no. 8, pp. 081 302-081302, May 2010.

[27] W. D. Callister and D. G. Rethwisch, Materials science and engineering: an introduction. Wiley New York, 2007, vol. 7.

[28] E. H. Nicollian, J. R. Brews, and E. H. Nicollian, MOS (metal oxide semiconductor) physics and technology. Wiley New York et al., 1982, vol. 1987.

[29] B. Svensson, S. Pearton, and C. Jagadish, Oxide Semiconductors, ser. Semiconductors and Semimetals. Elsevier Science, 2013.

[30] G. H. Kwei, A. C. Lawson, S. J. L. Billinge, and S. W. Cheong, "Structures of the ferroelectric phases of barium titanate," The Journal of Physical Chemistry, vol. 97, no. 10, pp. 2368-2377, 1993.

[31] W. J. Merz, "Domain formation and domain wall motions in ferroelectric $\mathrm{BaTiO}_{3}$ single crystals," Physical Review, vol. 95, no. 3, p. 690, 1954.

[32] N. Nuraje and K. Su, "Perovskite ferroelectric nanomaterials," Nanoscale, vol. 5, pp. 8752-8780, 2013.

[33] J. Smit and H. P. J. Wijn, Ferrites: physical properties of ferrimagnetic oxides in relation to their technical applications. Wiley, 1959.

[34] M. Julliere, "Tunneling between ferromagnetic films," Physics Letters A, vol. 54, no. 3, pp. 225 -226, 1975.

[35] S. Parkin, X. Jiang, C. Kaiser, A. Panchula, K. Roche, and M. Samant, "Magnetically engineered spintronic sensors and memory," Proceedings of the IEEE, vol. 91, no. 5, pp. 661-680, 2003.

[36] J. Coey, M Venkatesan, and C. Fitzgerald, "Donor impurity band exchange in dilute ferromagnetic oxides," Nature materials, vol. 4, no. 2, pp. 173-179, 2005.

[37] D. Wollman, D. Van Harlingen, W. Lee, D. Ginsberg, and A. Leggett, "Experimental determination of the superconducting pairing state in YBCO from the phase coherence of YBCO-Pb dc SQUIDs," Physical review letters, vol. 71, no. 13, p. 2134, 1993.

[38] G. Burns, High-temperature superconductivity. Academic Press San Diego, 1992.

[39] G Blatter, M. Feigel'Man, V. Geshkenbein, A. Larkin, and V. M. Vinokur, "Vortices in high-temperature superconductors," Reviews of Modern Physics, vol. 66, no. 4, p. 1125, 1994.

[40] F. Argall, "Switching phenomena in titanium oxide thin films," Solid-State Electronics, vol. 11, no. 5, pp. 535 -541, 1968.

[41] T. D. Lin, H. C. Chiu, P. Chang, L. T. Tung, C. P. Chen, M. Hong, J. Kwo, W. Tsai, and Y. C. Wang, "High-performance self-aligned inversion-channel $\mathrm{In}_{0} .53 \mathrm{Ga}_{0} .47 \mathrm{As}$ metal-oxide-semiconductor field-effect-transistor with $\mathrm{Al}_{2} \mathrm{O}_{3} \mathrm{Ga}_{2} \mathrm{O}_{3}\left(\mathrm{Gd}_{2} \mathrm{O}_{3}\right)$ as gate dielectrics," Applied Physics Letters, vol. 93, no. 3, pp. -, 2008.

[42] A. Majumdar, "Microscale heat conduction in dielectric thin films," Journal of Heat Transfer, vol. 115, no. 1, pp. 7-16, Feb. 1993. 
[43] J. McPherson and D. Baglee, "Acceleration factors for thin gate oxide stressing," in Reliability Physics Symposium, 1985. 23rd Annual, 1985, pp. 1-5.

[44] M. A. Panzer, M. Shandalov, J. A. Rowlette, Y. Oshima, Y. W. Chen, P. C. McIntyre, and K. E. Goodson, "Thermal properties of ultrathin hafnium oxide gate dielectric films," Electron Device Letters, IEEE, vol. 30, no. 12, pp. 1269-1271, 2009.

[45] S. Roberts, "Dielectric and piezoelectric properties of barium titanate," Phys. Rev., vol. 71, pp. 890-895, 12 1947.

[46] A. Hagfeldt and M. Gratzel, "Molecular photovoltaics," Accounts of Chemical Research, vol. 33, no. 5, pp. 269-277, 2000.

[47] L.-k. Tsui and G. Zangari, "Titania nanotubes by electrochemical anodization for solar energy conversion," Journal of The Electrochemical Society, vol. 161, no. 7, pp. D3066-D3077, 2014.

[48] L. E. Bell, "Cooling, heating, generating power, and recovering waste heat with thermoelectric systems," Science, vol. 321, no. 5895, pp. 1457-1461, 2008.

[49] W. Kim, "Strategies for engineering phonon transport in thermoelectrics," J. Mater. Chem. C, pp. -, 2015.

[50] J.-F. Li, W.-S. Liu, L.-D. Zhao, and M. Zhou, "High-performance nanostructured thermoelectric materials," NPG Asia Mater, vol. 2, pp. 152-158, Oct. 2010.

[51] J.-P. Niemela, A. Giri, P. E. Hopkins, and M. Karppinen, "Ultra-low thermal conductivity in $\mathrm{TiO}_{2}$ :C superlattices," J. Mater. Chem. A, vol. 3, pp. 11527-11 532, 212015.

[52] D. R. Gaskell, Introduction to the Thermodynamics of Materials. CRC Press, 2008.

[53] R. Swalin, Thermodynamics of solids, ser. Wiley series on the science and technology of materials. Wiley, 1962.

[54] A. von Hippel, "Ferroelectricity, domain structure, and phase transitions of barium titanate," Rev. Mod. Phys., vol. 22, pp. 221-237, 31950.

[55] I. H. Levin, "Synthesis of precious stones.," Journal of Industrial E Engineering Chemistry, vol. 5, no. 6, pp. 495-500, 1913.

[56] A. Cho and J. Arthur, "Molecular beam epitaxy," Progress in Solid State Chemistry, vol. 10, pp. 157 -191, 1975.

[57] W. D. Kingery, "Introduction to ceramics," 1960.

[58] C. J. Brinker and G. W. Scherer, Sol-gel science: the physics and chemistry of sol-gel processing. Academic press, 2013.

[59] G. H. Johnson, "Influence of impurities on electrical conductivity of rutile," Journal of the American Ceramic Society, vol. 36, no. 3, pp. 97-101, 1953.

[60] R. W. Millar, "The heat capacity at low temperatures of zinc oxide and of cadmium oxide1," Journal of the American Chemical Society, vol. 50, no. 10, pp. 2653-2656, 1928.

[61] K. A. McCarthy and S. S. Ballard, "New data on the thermal conductivity of optical crystals," J. Opt. Soc. Am., vol. 41, no. 12, pp. 1062-1063, 1951.

[62] G. A. Slack, "Thermal conductivity of $\mathrm{MgO}, \mathrm{Al}_{2} \mathrm{O}_{3}, \mathrm{MgAl}_{2} \mathrm{O}_{4}$, and $\mathrm{Fe}_{3} \mathrm{O}_{4}$ crystals from 3 to $300 \mathrm{~K}$," Phys. Rev., vol. 126, pp. 427-441, 21962.

[63] S. Srivastava and R. Singh, "Lattice vibrational spectra and grüneisen parameter of some metal oxides," Chemical Physics Letters, vol. 7, no. 3, pp. $377-381,1970$.

[64] H. Siebeneck, W. P. Minnear, R. C. Bradt, and D. P. H. Hasselman, "Thermal diffusivity of nonstoichiometric titanium dioxide," Journal of the American Ceramic Society, vol. 59, no. 1-2, pp. 84-84, 1976.

[65] P. G. Klemens, "Theory of heat conduction in nonstoichiometric oxides and carbides," High Temperatures - High Pressures, vol. 17 , no. 1 , pp. $41-45,1985$.

[66] J. D. Freire and R. S. Katiyar, "Lattice dynamics of crystals with tetragonal $\mathrm{BaTiO}_{3}$ structure," Phys. Rev. B, vol. 37, pp. 2074-2085, Feb. 1988.

[67] D. R. Clarke and S. R. Phillpot, "Thermal barrier coating materials," Materials Today, vol. 8, no. 6, pp. 22 -29, 2005. 
[68] M. R. Winter and D. R. Clarke, "Oxide materials with low thermal conductivity," Journal of the American Ceramic Society, vol. 90, no. 2, pp. 533-540, 2007.

[69] M. Tachibana, T. Kolodiazhnyi, and E. Takayama-Muromachi, "Thermal conductivity of perovskite ferroelectrics," Applied Physics Letters, vol. 93, no. 9, 092902, p. 092 902, 2008.

[70] Y. Suemune, "Thermal conductivity of $\mathrm{BaTiO}_{3}$ and $\mathrm{SrTiO}_{3}$ from $4.5 \mathrm{~K}$ to $300 \mathrm{~K}$," Journal of the Physical Society of Japan, vol. 20, no. 1, pp. 174-175, 1965.

[71] A. Mante and J. Volger, "The thermal conductivity of $\mathrm{BaTiO}_{3}$ in the neighbourhood of its ferroelectric transition temperatures," Physics Letters A, vol. 24, no. 3, pp. 139 -140, 1967.

[72] M. Tachibana and E. Takayama-Muromachi, "Thermal conductivity and heat capacity of the relaxor ferroelectric pmnpt," Phys. Rev. B, vol. 79, p. 100 104, 102009.

[73] M. Tachibana, K. Sasame, H. Kawaji, T. Atake, and E. Takayama-Muromachi, "Thermal signatures of nanoscale inhomogeneities and ferroelectric order in PZN-PT," Phys. Rev. B, vol. 80, p. 094 115, 92009.

[74] A. Knudsen, S. Delzer, and C. Langhoff, Laser flash thermal conductivity apparatus and method, US Patent 4,928,254, 1990.

[75] J. Callaway, "Model for lattice thermal conductivity at low temperatures," Phys. Rev., vol. 113, pp. 1046-1051, 4 1959.

[76] J. Callaway and H. C. von Baeyer, "Effect of point imperfections on lattice thermal conductivity," Phys. Rev., vol. 120, pp. 1149-1154, 41960.

[77] J. Callaway, "Theory of scattering in solids," Journal of Mathematical Physics, vol. 5, no. 6, pp. 783-798, 1964.

[78] P. G. Klemens, "The scattering of low-frequency lattice waves by static imperfections," Proceedings of the Physical Society. Section A, vol. 68, no. 12, p. 1113, 1955.

[79] P. G. Klemens, "Thermal resistance due to point defects at high temperatures," Phys. Rev., vol. 119, pp. 507-509, 2 1960.

[80] C. Ratsifaritana and P. Klemens, "Scattering of phonons by vacancies," English, International Journal of Thermophysics, vol. 8, no. 6, pp. $737-750,1987$.

[81] P. G. Klemens, "Phonon scattering by oxygen vacancies in ceramics," Physica B: Condensed Matter, vol. 263-264, no. 0, pp. $102-104,1999$.

[82] P. Klemens, "Theory of thermal conduction in thin ceramic films," English, International Journal of Thermophysics, vol. 22 , no. 1 , pp. $265-275,2001$.

[83] M. Sangster, G Peckham, and D. Saunderson, "Lattice dynamics of magnesium oxide," Journal of Physics C: Solid State Physics, vol. 3, no. 5, p. 1026, 1970.

[84] G. Lewis and C. Catlow, "Potential models for ionic oxides," Journal of Physics C: Solid State Physics, vol. 18, no. 6, p. $1149,1985$.

[85] P. K. Naicker, P. T. Cummings, H. Zhang, and J. F. Banfield, "Characterization of titanium dioxide nanoparticles using molecular dynamics simulations," The Journal of Physical Chemistry B, vol. 109, no. 32, pp. 15 243-15249, 2005.

[86] Z. Zheng, X. Chen, B. Deng, A. Chernatynskiy, S. Yang, L. Xiong, and Y. Chen, "Phonon thermal transport through tilt grain boundaries in strontium titanate," Journal of Applied Physics, vol. 116, no. 7, 073706, 2014.

[87] P. Thibaudeau and F. Gervais, "Ab initio investigation of phonon modes in the $\mathrm{MgAl}_{2} \mathrm{O}_{4}$ spinel," Journal of Physics: Condensed Matter, vol. 14, no. 13, p. 3543, 2002.

[88] D. Gryaznov, E. Blokhin, A. Sorokine, E. A. Kotomin, R. A. Evarestov, A. Bussmann-Holder, and J. Maier, "A comparative ab initio thermodynamic study of oxygen vacancies in $\mathrm{ZnO}$ and $\mathrm{SrTiO}_{3}$ : emphasis on phonon contribution," The Journal of Physical Chemistry C, vol. 117, no. 27, pp. 13776-13 784, 2013.

[89] D. G. Cahill, "Thermal conductivity measurement from 30 to $750 \mathrm{~K}$ : the $3 \omega$ method," Review of Scientific Instruments, vol. 61 , no. 2, pp. 802-808, 1990. 
[90] D. G. Cahill, J. R. Olson, H. E. Fischer, S. K. Watson, R. B. Stephens, R. H. Tait, T. Ashworth, and R. O. Pohl, "Thermal conductivity and specific heat of glass ceramics," Phys. Rev. B, vol. 44, pp. 12 226-12 232, 221991.

[91] K. Takahata, Y. Iguchi, D. Tanaka, T. Itoh, and I. Terasaki, "Low thermal conductivity of the layered oxide $(\mathrm{Na}, \mathrm{Ca}) \mathrm{Co}_{2} \mathrm{O}_{4}$ : another example of a phonon glass and an electron crystal," Phys. Rev. B, vol. 61, pp. 12 551-12 555, 19 2000 .

[92] S. Wiedigen, T. Kramer, M. Feuchter, I. Knorr, N. Nee, J. Hoffmann, M. Kamlah, C. A. Volkert, and C. Jooss, "Interplay of point defects, biaxial strain, and thermal conductivity in homoepitaxial $\mathrm{SrTiO}_{3}$ thin films," Applied Physics Letters, vol. 100, no. 6, 061904, 2012.

[93] B. M. Foley, H. J. Brown-Shaklee, M. J. Campion, D. L. Medlin, P. G. Clem, J. F. Ihlefeld, and P. E. Hopkins, "Glass-like thermal conductivity of (010)-textured lanthanum-doped strontium niobate synthesized with wet chemical deposition," Journal of the American Ceramic Society, 2014.

[94] B. M. Foley, H. J. Brown-Shaklee, J. C. Duda, R. Cheaito, B. J. Gibbons, D. Medlin, J. F. Ihlefeld, and P. E. Hopkins, "Thermal conductivity of nano-grained $\mathrm{SrTiO}_{3}$ thin films," Applied Physics Letters, vol. 101, no. 23, pp. 231 908-2319084, 2012 .

[95] B. F. Donovan, B. M. Foley, J. F. Ihlefeld, J.-P. Maria, and P. E. Hopkins, "Spectral phonon scattering effects on the thermal conductivity of nano-grained barium titanate," Applied Physics Letters, vol. 105, no. 8, 082907, p. $082907,2014$.

[96] Y. Toyoshima, H. Iwai, F. Matsuoka, H. Hayashida, K. Maeguchi, and K. Kanzaki, "Analysis on gate-oxide thickness dependence of hot-carrier-induced degradation in thin-gate oxide nmosfet's," Electron Devices, IEEE Transactions on, vol. 37 , no. 6 , pp. $1496-1503,1990$

[97] D Arnold, E Cartier, and D. DiMaria, "Acoustic-phonon runaway and impact ionization by hot electrons in silicon dioxide," Physical Review B, vol. 45, no. 3, p. 1477, 1992.

[98] L. T. Su, J. E. Chung, D. A. Antoniadis, K. E. Goodson, and M. I. Flik, "Measurement and modeling of self-heating in soi nmosfet's," Electron Devices, IEEE Transactions on, vol. 41, no. 1, pp. 69-75, 1994.

[99] B. H. Lee, L. Kang, R. Nieh, W.-J. Qi, and J. C. Lee, "Thermal stability and electrical characteristics of ultrathin hafnium oxide gate dielectric reoxidized with rapid thermal annealing," Applied Physics Letters, vol. 76, no. 14, pp. 1926-1928, 2000 .

[100] X. Zhao and D. Vanderbilt, "Phonons and lattice dielectric properties of zirconia," Physical Review B, vol. 65, no. 7, p. $075105,2002$.

[101] N Bresson, S Cristoloveanu, C Mazuré, F Letertre, and H. Iwai, "Integration of buried insulators with high thermal conductivity in soi mosfets: thermal properties and short channel effects," Solid-state electronics, vol. 49, no. 9, pp. 1522$1528,2005$.

[102] K. Maitra, M. M. Frank, V. Narayanan, V. Misra, and E. A. Cartier, "Impact of metal gates on remote phonon scattering in titanium nitride/hafnium dioxide n-channel metal-oxide-semiconductor field effect transistors-low temperature electron mobility study," Journal of Applied Physics, vol. 102, no. 11, 114507, 2007.

[103] X Hong, A Posadas, K Zou, C. Ahn, and J Zhu, "High-mobility few-layer graphene field effect transistors fabricated on epitaxial ferroelectric gate oxides," Physical review letters, vol. 102, no. 13, p. $136808,2009$.

[104] K. Zou, X. Hong, D. Keefer, and J. Zhu, "Deposition of high-quality $\mathrm{HfO}_{2}$ on graphene and the effect of remote oxide phonon scattering," Physical review letters, vol. 105, no. 12, p. 126601, 2010.

[105] D. Abraham and P. Trouilloud, Thermally-assisted magnetic random access memory (MRAM), US Patent 6,385,082, 2002 .

[106] I. L. Prejbeanu, M Kerekes, R. C. Sousa, H Sibuet, O Redon, B Dieny, and J. P. Nozieres, "Thermally assisted MRAM," Journal of Physics: Condensed Matter, vol. 19, no. 16, p. 165 218, 2007. 
[107] D. R. Clarke, "Materials selection guidelines for low thermal conductivity thermal barrier coatings," Surface and Coatings Technology, vol. 163-164, no. 0, pp. $67-74,2003$, Proceedings of the 29th International conference on Metallurgical Coatings and Thin Films.

[108] K. Schlichting, N. Padture, and P. Klemens, "Thermal conductivity of dense and porous yttria-stabilized zirconia," English, Journal of Materials Science, vol. 36, no. 12, pp. 3003-3010, 2001.

[109] P. K. Schelling and S. R. Phillpot, "Mechanism of thermal transport in zirconia and yttria-stabilized zirconia by moleculardynamics simulation," Journal of the American Ceramic Society, vol. 84, no. 12, pp. 2997-3007, 2001.

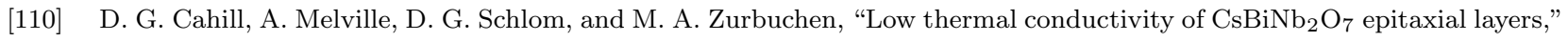
Applied Physics Letters, vol. 96, no. 12, 121903, 2010.

[111] Y. Wang, K. Fujinami, R. Zhang, C. Wan, N. Wang, Y. Ba, and K. Koumoto, "Interfacial thermal resistance and thermal conductivity in nanograined srtio 3," Applied Physics Express, vol. 3, no. 3, p. 031 101, 2010.

[112] M. N. Khan, H.-T. Kim, H. Minami, and H. Uwe, "Thermoelectric properties of niobium doped hexagonal barium titanate," Materials Letters, vol. 47, no. 1-2, pp. $95-101,2001$.

[113] T. C. Harman, P. J. Taylor, M. P. Walsh, and B. E. LaForge, "Quantum dot superlattice thermoelectric materials and devices," Science, vol. 297, no. 5590, pp. 2229-2232, 2002.

[114] W. Kim, J. Zide, A. Gossard, D. Klenov, S. Stemmer, A. Shakouri, and A. Majumdar, "Thermal conductivity reduction and thermoelectric figure of merit increase by embedding nanoparticles in crystalline semiconductors," Phys. Rev. Lett., vol. 96, p. $045901,42006$.

[115] M. S. Dresselhaus, G. Chen, M. Y. Tang, R. G. Yang, H. Lee, D. Z. Wang, Z. F. Ren, J.-P. Fleurial, and P. Gogna, "New directions for low-dimensional thermoelectric materials," Advanced Materials, vol. 19, no. 8, pp. 1043-1053, 2007.

[116] J. He, J. R. Sootsman, S. N. Girard, J.-C. Zheng, J. Wen, Y. Zhu, M. G. Kanatzidis, and V. P. Dravid, "On the origin of increased phonon scattering in nanostructured pbte based thermoelectric materials," Journal of the American Chemical Society, vol. 132, no. 25, pp. 8669-8675, 2010, PMID: 20524606.

[117] C. J. Vineis, A. Shakouri, A. Majumdar, and M. G. Kanatzidis, "Nanostructured thermoelectrics: big efficiency gains from small features," Advanced Materials, vol. 22, no. 36, pp. 3970-3980, 2010.

[118] J. Tang, H.-T. Wang, D. H. Lee, M. Fardy, Z. Huo, T. P. Russell, and P. Yang, "Holey silicon as an efficient thermoelectric material," Nano Letters, vol. 10, no. 10, pp. 4279-4283, 2010.

[119] J. Ravichandran, W. Siemons, D.-W. Oh, J. T. Kardel, A. Chari, H. Heijmerikx, M. L. Scullin, A. Majumdar, R. Ramesh, and D. G. Cahill, "High-temperature thermoelectric response of double-doped $\mathrm{SrTiO}_{3}$ epitaxial films," Phys. Rev. B, vol. 82, no. 16, 165126, p. 165126 , Oct. 2010.

[120] A. X. Levander, T. Tong, K. M. Yu, J. Suh, D. Fu, R. Zhang, H. Lu, W. J. Schaff, O. Dubon, W. Walukiewicz, D. G. Cahill, and J. Wu, "Effects of point defects on thermal and thermoelectric properties of inn," Applied Physics Letters, vol. 98, no. 1, 012108, 2011.

[121] S. R. Sarath Kumar, A. I. Abutaha, M. N. Hedhili, and H. N. Alshareef, "Effect of oxygen vacancy distribution on the thermoelectric properties of La-doped $\mathrm{SrTiO}_{3}$ epitaxial thin films," Journal of Applied Physics, vol. 112, no. 11, 114104, 2012.

[122] V. Kessler, D. Gautam, T. Hülser, M. Spree, R. Theissmann, M. Winterer, H. Wiggers, G. Schierning, and R. Schmechel, "Thermoelectric properties of nanocrystalline silicon from a scaled-up synthesis plant," Advanced Engineering Materials, vol. 15, no. 5, pp. 379-385, 2013.

[123] Y. Yan and J. A. Malen, "Periodic heating amplifies the efficiency of thermoelectric energy conversion," Energy Environ. Sci., vol. 6, pp. 1267-1273, 42013. 
[124] J.-H. Bahk and A. Shakouri, "Enhancing the thermoelectric figure of merit through the reduction of bipolar thermal conductivity with heterostructure barriers," Applied Physics Letters, vol. 105, no. 5, 052106, 2014.

[125] K. M. Hoogeboom-Pot, J. N. Hernandez-Charpak, X. Gu, T. D. Frazer, E. H. Anderson, W. Chao, R. W. Falcone, R. Yang, M. M. Murnane, H. C. Kapteyn, and D. Nardi, "A new regime of nanoscale thermal transport: collective diffusion increases dissipation efficiency," Proceedings of the National Academy of Sciences, 2015.

[126] J. Loureiro, T. Mateus, S. Filonovich, M. Ferreira, J. Figueira, A. Rodrigues, B. F. Donovan, P. Hopkins, and I. Ferreira, "Hydrogenated nanocrystalline silicon thin films with promising thermoelectric properties," Applied Physics A, pp. 1-6, 2015.

[127] J.-L. Lan, Y. Liu, Y.-H. Lin, C.-W. Nan, Q. Cai, and X. Yang, "Enhanced thermoelectric performance of $\mathrm{In}_{2} \mathrm{O}_{3}-$-based ceramics via nanostructuring and point defect engineering," Sci. Rep., vol. 5, Jan. 2015.

[128] S. O. Kasap, Principles of electronic materials and devices. McGraw-Hill, 2006.

[129] R. Franz and G. Wiedemann, "Ueber die warme-leitungsfahigkeit der metalle," Annalen der Physik, vol. 165, no. 8, pp. 497-531, 1853.

[130] J. Yahia, "Dependence of the electrical conductivity and thermoelectric power of pure and aluminum-doped rutile on equilibrium oxygen pressure and temperature," Phys. Rev., vol. 130, pp. 1711-1719, 51963.

[131] H. Wang, C. Wang, W. Su, J. Liu, Y. Zhao, H. Peng, J. Zhang, M. Zhao, J. Li, N. Yin, and L. Mei, "Enhancement of thermoelectric figure of merit by doping Dy in $\mathrm{La}_{0} \cdot 1 \mathrm{Sr}_{0} .9 \mathrm{TiO}_{3}$ ceramic," Materials Research Bulletin, vol. 45, no. 7 , pp. $809-812,2010$.

[132] S. Bhattacharya, A. Mehdizadeh Dehkordi, S. Tennakoon, R. Adebisi, J. R. Gladden, T. Darroudi, H. N. Alshareef, and T. M. Tritt, "Role of phonon scattering by elastic strain field in thermoelectric $\operatorname{Sr}_{1 x} \mathrm{Y}_{x} \mathrm{TiO} 3 \delta$," Journal of Applied Physics, vol. 115, no. 22, pp. -, 2014.

[133] J. Tang, W. Wang, G.-L. Zhao, and Q. Li, "Colossal positive seebeck coefficient and low thermal conductivity in reduced $\mathrm{TiO}_{2}$," Journal of Physics: Condensed Matter, vol. 21, no. 20, p. 205 703, 2009.

[134] S. Harada, K. Tanaka, and H. Inui, "Thermoelectric properties and crystallographic shear structures in titanium oxides of the magneli phases," Journal of Applied Physics, vol. 108, no. 8, 083703, pp. -, 2010.

[135] N. Mingo, D. Hauser, N. P. Kobayashi, M. Plissonnier, and A. Shakouri, "“"nanoparticle-in-alloy" approach to efficient thermoelectrics: silicides in SiGe," Nano Letters, vol. 9, no. 2, pp. 711-715, 2009.

[136] A. Vassighi and M. Sachdev, Thermal and Power Management of Integrated Circuits, ser. Integrated Circuits and Systems. Springer US, 2006.

[137] C. Dames, "Solid-state thermal rectification with existing bulk materials," Journal of Heat Transfer, vol. 131, no. 6, pp. $061301-061301$, Mar. 2009.

[138] C. W. Chang, D. Okawa, A. Majumdar, and A. Zettl, "Solid-state thermal rectifier," Science, vol. 314, no. 5802, pp. 1121$1124,2006$.

[139] M. N. Luckyanova, D. Chen, W. Ma, H. L. Tuller, G. Chen, and B. Yildiz, "Thermal conductivity control by oxygen defect concentration modification in reducible oxides: the case of $\operatorname{Pr}_{0} \cdot 1 \mathrm{Ce}_{0} .9 \mathrm{O}_{2}$ thin films," Applied Physics Letters, vol. 104, no. 6, p. $061911,2014$.

[140] C. M. Brooks, R. B. Wilson, A. Schafer, J. A. Mundy, M. E. Holtz, D. A. Muller, J. Schubert, D. G. Cahill, and D. G. Schlom, "Tuning thermal conductivity in homoepitaxial $\mathrm{SrTiO}_{3}$ films via defects," Applied Physics Letters, vol. 107 , no. 5 , pp.,- 2015.

[141] S. N. Ruddlesden and P. Popper, "New compounds of the $\mathrm{K}_{2} \mathrm{NiF}_{4}$ type," Acta Crystallographica, vol. 10, no. 8, pp. 538$539,1957$.

[142] — "The compound $\mathrm{Sr}_{3} \mathrm{Ti}_{2} \mathrm{O}_{7}$ and its structure," Acta Crystallographica, vol. 11, no. 1, pp. 54-55, 1958. 
[143] E. Sachet, C. T. Shelton, J. S. Harris, B. E. Gaddy, D. L. Irving, S. Curtarolo, B. F. Donovan, P. E. Hopkins, P. A. Sharma, A. L. Sharma, J. Ihlefeld, S. Franzen, and J.-P. Maria, "Dysprosium-doped cadmium oxide as a gateway material for mid-infrared plasmonics," Nature Materials, vol. 14, no. 4, pp. 414-420, Apr. 2015.

[144] B. F. Donovan, E. Sachet, J.-P. Maria, and P. E. Hopkins, "Interplay between mass-impurity and vacancy phonon scattering effects on the thermal conductivity of doped cadmium oxide," Applied Physics Letters, vol. 108, no. 2, 021901, 2016.

[145] J. F. Ihlefeld, A. M. Vodnick, S. P. Baker, W. J. Borland, and J.-P. Maria, "Extrinsic scaling effects on the dielectric response of ferroelectric thin films," Journal of Applied Physics, vol. 103, no. 7, 074112, p. 074 112, 2008.

[146] G. L. Brennecka, J. F. Ihlefeld, J.-P. Maria, B. A. Tuttle, and P. G. Clem, "Processing technologies for high-permittivity thin films in capacitor applications," Journal of the American Ceramic Society, vol. 93, no. 12, pp. 3935-3954, 2010.

[147] J. Ravichandran, A. K. Yadav, R. Cheaito, P. B. Rossen, A. Soukiassian, S. J. Suresha, J. C. Duda, B. M. Foley, C.-H. Lee, Y. Zhu, A. W. Lichtenberger, J. E. Moore, D. A. Muller, D. G. Schlom, P. E. Hopkins, A. Majumdar, R. Ramesh, and M. A. Zurbuchen, "Crossover from incoherent to coherent phonon scattering in epitaxial oxide superlattices," Nat Mater, vol. advance online publication, p. 3826, Dec. 2013.

[148] Y. Shen, D. R. Clarke, and P. A. Fuierer, "Anisotropic thermal conductivity of the aurivillus phase, bismuth titanate $\left(\mathrm{Bi}_{4} \mathrm{Ti}_{3} \mathrm{O}_{1} 2\right)$ : a natural nanostructured superlattice," Applied Physics Letters, vol. 93, no. 10, 2008.

[149] N. Roberts and D. Walker, "A review of thermal rectification observations and models in solid materials," International Journal of Thermal Sciences, vol. 50, no. 5, pp. 648-662, 2011.

[150] W. Kobayashi, Y. Teraoka, and I. Terasaki, "An oxide thermal rectifier," Applied Physics Letters, vol. 95, no. 17, 171905, 2009.

[151] Y. Wang, A. Vallabhaneni, J. Hu, B. Qiu, Y. P. Chen, and X. Ruan, "Phonon lateral confinement enables thermal rectification in asymmetric single-material nanostructures," Nano Letters, vol. 14, no. 2, pp. 592-596, 2014, PMID: 24393070.

[152] R. Chen, Y. Cui, H. Tian, R. Yao, Z. Liu, Y. Shu, C. Li, Y. Yang, T. Ren, G. Zhang, and R. Zou, "Controllable thermal rectification realized in binary phase change composites," Scientific Reports, vol. 5, 8884 EP -, Mar. 2015.

[153] J. W. Lynn, H. G. Smith, and R. M. Nicklow, "Lattice dynamics of gold," Phys. Rev. B, vol. 8, pp. 3493-3499, 8 1973.

[154] J. G. Traylor, H. G. Smith, R. M. Nicklow, and M. K. Wilkinson, "Lattice dynamics of rutile," Phys. Rev. B, vol. 3, pp. 3457-3472, 101971.

[155] M. G. Holland, "Analysis of lattice thermal conductivity," Phys. Rev., vol. 132, pp. 2461-2471, 61963.

[156] J. Chung, A. McGaughey, and M Kaviany, "Role of phonon dispersion in lattice thermal conductivity modeling," Journal of heat transfer, vol. 126, no. 3, pp. 376-380, 2004.

[157] E. Pop, R. W. Dutton, and K. E. Goodson, "Analytic band monte carlo model for electron transport in si including acoustic and optical phonon dispersion," Journal of Applied Physics, vol. 96, no. 9, pp. 4998-5005, 2004.

[158] P. Giannozzi, S. de Gironcoli, P. Pavone, and S. Baroni, "Ab initio calculation of phonon dispersions in semiconductors," Phys. Rev. B, vol. 43, pp. 7231-7242, 91991.

[159] P. Ghosez, E. Cockayne, U. V. Waghmare, and K. M. Rabe, "Lattice dynamics of $\mathrm{BaTiO}_{3}, \mathrm{PbTiO}_{3}$, and $\mathrm{PbZrO}_{3}$ : a comparative first-principles study," Phys. Rev. B, vol. 60, pp. 836-843, 21999.

[160] B. Liao, B. Qiu, J. Zhou, S Huberman, K Esfarjani, and G. Chen, "Significant reduction of lattice thermal conductivity by electron-phonon interaction in silicon with high carrier concentrations: a first-principles study," Phys. Rev. Lett., vol. 114, p. $115901,2015$.

[161] P. E. Hopkins, C. Adamo, L. Ye, B. D. Huey, S. R. Lee, D. G. Schlom, and J. F. Ihlefeld, "Effects of coherent ferroelastic domain walls on the thermal conductivity and kapitza conductance in bismuth ferrite," Applied Physics Letters, vol. 102, no. $12,2013$. 
[162] M. A. Weilert, M. E. Msall, A. C. Anderson, and J. P. Wolfe, "Phonon scattering from ferroelectric domain walls: phonon imaging in KDP," Phys. Rev. Lett., vol. 71, pp. 735-738, 51993.

[163] A. Mante and J. Volger, "Phonon transport in barium titanate," Physica, vol. 52, no. 4, pp. 577 -604, 1971.

[164] K. Sinha and U. Upadhyaya, "Phonon-magnon interaction in magnetic crystals," Physical Review, vol. 127, no. 2, p. 432, 1962.

[165] G. T. Hohensee, R. Wilson, J. P. Feser, and D. G. Cahill, "Magnon-phonon coupling in the spin-ladder compound $\mathrm{Ca}_{9} \mathrm{La}_{5} \mathrm{Cu}_{2} 4 \mathrm{O}_{4} 1$ measured by time-domain thermoreflectance," Physical Review B, vol. 89, no. 2, p. $024422,2014$.

[166] M. Asheghi, Y. K. Leung, S. S. Wong, and K. E. Goodson, "Phonon-boundary scattering in thin silicon layers," Applied Physics Letters, vol. 71, no. 13, pp. 1798-1800, 1997.

[167] R Berman, "The thermal conductivity of some polycrystalline solids at low temperatures," Proceedings of the Physical Society. Section A, vol. 65, no. 12, p. 1029, 1952.

[168] B. Abeles, "Lattice thermal conductivity of disordered semiconductor alloys at high temperatures," Phys. Rev., vol. 131, pp. 1906-1911, 51963.

[169] R. E. Peierls, Quantum theory of solids, 23. Oxford University Press, 1955.

[170] J. W. Strutt, "On the scattering of light by small particles," in Scientific Papers, vol. 1, Cambridge Books Online, Cambridge University Press, 1899, pp. 104-110.

[171] P. Klemens, "Phonon scattering and thermal resistance due to grain boundaries," International Journal of Thermophysics, vol. 15, no. 6, pp. 1345-1351, 1994.

[172] P. E. Hopkins, P. M. Norris, and R. J. Stevens, "Influence of inelastic scattering at metal-dielectric interfaces," Journal of Heat Transfer, vol. 130, no. 2, pp. 022 401-022 401, Feb. 2008.

[173] L. Boltzmann, "Zur theorie der elastischen nachwirkung," Annalen der Physik, vol. 241, no. 11, pp. 430-432, 1878.

[174] A. de Moivre, The doctrine of chances. H. Woodfall, 1738.

[175] P. Tzanetakis, J. Hillairet, and G. Revel, "The formation energy of vacancies in aluminium and magnesium," physica status solidi (b), vol. 75, no. 2, pp. 433-439, 1976.

[176] F. Kroger and H. Vink, "Relations between the concentrations of imperfections in crystalline solids," in, ser. Solid State Physics, F. Seitz and D. Turnbull, Eds., vol. 3, Academic Press, 1956, pp. $307-435$.

[177] N. Tallan, Electrical conductivity in ceramics and glass, ser. Electrical Conductivity in Ceramics and Glass v. 1. M. Dekker, 1974.

[178] S. Andersson, B. Collen, U. Kuylenstierna, A. Magnali, H Pestmalis, and S Asbrink, "Phase analysis studies on the titanium-oxygen system," Acta Chemica Scandinavica, vol. 11, no. 10, pp. 1641-1652, 1957.

[179] P. Waldner and G. Eriksson, "Thermodynamic modelling of the system titanium-oxygen," Calphad, vol. 23, no. 2, pp. 189 $-218,1999$.

[180] H. Okamoto, "O-Ti (oxygen-titanium)," Journal of Phase Equilibria and Diffusion, vol. 32, no. 5, pp. 473-474, 2011.

[181] G. J. Wood and L. A. Bursill, "The formation energy of crystallographic shear planes in $\operatorname{Ti}_{n} \mathrm{O}_{2 n-1}$," Proceedings of the Royal Society of London A: Mathematical, Physical and Engineering Sciences, vol. 375, no. 1760, pp. 105-125, 1981.

[182] L. A. Bursill, B. G. Hyde, O. Terasaki, and D. Watanabe, "On a new family of titanium oxides and the nature of slightly-reduced rutile," Philosophical Magazine, vol. 20, no. 164, pp. 347-359, 1969.

[183] L. Liborio and N. Harrison, "Thermodynamics of oxygen defective Magneli phases in rutile: a first-principles study," Phys. Rev. B, vol. 77, p. $104104,102008$.

[184] L. Bursill and B. Hyde, "Crystallographic shear in the higher titanium oxides: structure, texture, mechanisms and thermodynamics," Progress in Solid State Chemistry, vol. 7, pp. 177 -253, 1972. 
[185] A. A. Gusev, E. G. Avvakumov, A. Z. Medvedev, and A. I. Masliy, "Ceramic electrodes based on magneli phases of titanium oxides," Science of Sintering, vol. 39, 2007.

[186] L. A. Bursill and B. G. Hyde, "On the aggregation of wadsley defects in slightly reduced rutile," Philosophical Magazine, vol. 23, no. 181, pp. 3-15, 1971.

[187] L. A. Bursill, B. G. Hyde, and D. K. Philp, "New crystallographic shear families derived from the rutile structure, and the possibility of continuous ordered solid solution," Philosophical Magazine, vol. 23, no. 186, pp. 1501-1513, 1971.

[188] M. Aono and R. R. Hasiguti, "Interaction and ordering of lattice defects in oxygen-deficient rutile $\mathrm{TiO}_{2-x}$," Phys. Rev. $B$, vol. 48, pp. 12 406-12 414, 171993.

[189] Y. L. Page and P. Strobel, "Structural chemistry of magneli phases $\mathrm{Ti}_{n} \mathrm{O}_{2 n 1}$. i. cell and structure comparisons," Journal of Solid State Chemistry, vol. 43, no. 3, pp. $314-319,1982$.

[190] C. Tang, D. Zhou, and Q. Zhang, "Synthesis and characterization of magneli phases: reduction of $\mathrm{TiO}_{2}$ in a decomposed $\mathrm{NH}_{3}$ atmosphere," Materials Letters, vol. 79, pp. 42 -44, 2012.

[191] E. Finazzi, C. D. Valentin, and G. Pacchioni, "Nature of Ti interstitials in reduced bulk anatase and rutile $\mathrm{TiO}_{2}$," The Journal of Physical Chemistry C, vol. 113, no. 9, pp. 3382-3385, 2009.

[192] C. Acha, M. Monteverde, M. Nunez-Regueiro, A. Kuhn, and M. A. Alario Franco, "Electrical resistivity of the $\mathrm{Ti}_{4} \mathrm{O}_{7}$ magneli phase under high pressure," The European Physical Journal B - Condensed Matter and Complex Systems, vol. 34 , no. 4, pp. 421-428, 2003.

[193] W. Heckel, M. Wehlau, S. B. Maisel, T. Frauenheim, J. M. Knaup, and S. Müller, "How the aggregation of oxygen vacancies in rutile-based $\mathrm{TiO}_{2-\delta}$ phases causes memristive behavior," Phys. Rev. B, vol. 92, p. 214 104, 212015.

[194] K. Yoshida, T. Kawai, T. Nambara, S. Tanemura, K. Saitoh, and N. Tanaka, "Direct observation of oxygen atoms in rutile titanium dioxide by spherical aberration corrected high-resolution transmission electron microscopy," Nanotechnology, vol. 17, no. 15 , p. $3944,2006$.

[195] D.-H. Kwon, K. M. Kim, J. H. Jang, J. M. Jeon, M. H. Lee, G. H. Kim, X.-S. Li, G.-S. Park, B. Lee, S. Han, M. Kim, and C. S. Hwang, "Atomic structure of conducting nanofilaments in $\mathrm{TiO}_{2}$ resistive switching memory," Nat Nano, vol. 5, no. 2, pp. 148-153, Feb. 2010.

[196] R. Ciancio, E. Carlino, G. Rossi, C. Aruta, U. Scotti di Uccio, A. Vittadini, and A. Selloni, "Magneli-like phases in epitaxial anatase $\mathrm{TiO}_{2}$ thin films," Phys. Rev. B, vol. 86, p. 104110, 102012.

[197] A. Moballegh and E. C. Dickey, "Electric-field-induced point defect redistribution in single-crystal $\mathrm{TiO}_{2-x}$ and effects on electrical transport," Acta Materialia, vol. 86, pp. 352 -360, 2015.

[198] M. Marezio, D. McWhan, P. Dernier, and J. Remeika, "Structural aspects of the metal-insulator transitions in $\mathrm{Ti}_{4} \mathrm{O}_{7}$," Journal of Solid State Chemistry, vol. 6, no. 2, pp. 213 -221, 1973.

[199] T. Ioroi, Z. Siroma, N. Fujiwara, S.-i. Yamazaki, and K. Yasuda, "Sub-stoichiometric titanium oxide-supported platinum electrocatalyst for polymer electrolyte fuel cells," Electrochemistry Communications, vol. 7, no. 2, pp. 183 -188, 2005.

[200] T. Ioroi, H. Senoh, S.-i. Yamazaki, Z. Siroma, N. Fujiwara, and K. Yasuda, "Stability of corrosion-resistant magneli-phase $\mathrm{Ti}_{4} \mathrm{O}_{7}$-supported PEMFC catalysts at high potentials," Journal of The Electrochemical Society, vol. 155, no. 4, B321B326, 2008 .

[201] J. Graves, D. Pletcher, R. Clarke, and F. Walsh, "The electrochemistry of magneli phase titanium oxide ceramic electrodes part I. the deposition and properties of metal coatings," English, Journal of Applied Electrochemistry, vol. 21, no. 10, pp. 848-857, 1991.

[202] F. Walsh and R. Wills, "The continuing development of magneli phase titanium sub-oxides and Ebonex electrodes," Electrochimica Acta, vol. 55, no. 22, pp. 6342 -6351, 2010. 
[203] D. Regonini, V. Adamaki, C. Bowen, S. Pennock, J. Taylor, and A. Dent, "AC electrical properties of $\mathrm{TiO}_{2}$ and magneli phases, $\mathrm{Ti}_{n} \mathrm{O}_{2 n 1}$," Solid State Ionics, vol. 229, pp. 38 -44, 2012.

[204] C. He, S. Chang, X. Huang, Q. Wang, A. Mei, and P. K. Shen, "Direct synthesis of pure single-crystalline magneli phase $\mathrm{Ti}_{8} \mathrm{O}_{15}$ nanowires as conductive carbon-free materials for electrocatalysis," Nanoscale, vol. 7, pp. 2856-2861, 7 2015.

[205] L. Hao, Y. Lu, Y. Matsuda, K. Sagara, T. Otomitsu, and H. Yoshida, "Fabrication and thermoelectric properties of magneli phases by adding Ti into $\mathrm{TiO}_{2}$," in Advanced Materials, ICAMMP 2011, ser. Advanced Materials Research, vol. 415, Trans Tech Publications, Feb. 2012, pp. 1291-1296.

[206] M. Backhaus-Ricoult, J. R. Rustad, D. Vargheese, I. Dutta, and K. Work, "Levers for thermoelectric properties in titaniabased ceramics," Journal of Electronic Materials, vol. 41, no. 6, pp. 1636-1647, 2012.

[207] D. Portehault, V. Maneeratana, C. Candolfi, N. Oeschler, I. Veremchuk, Y. Grin, C. Sanchez, and M. Antonietti, "Facile general route toward tunable magneli nanostructures and their use as thermoelectric metal oxide/carbon nanocomposites," ACS Nano, vol. 5, no. 11, pp. 9052-9061, 2011, PMID: 21978378.

[208] C. Zener, "A theory of the electrical breakdown of solid dielectrics," Proceedings of the Royal Society of London. Series A, Containing Papers of a Mathematical and Physical Character, vol. 145, no. 855, pp. 523-529, 1934.

[209] S Whitehead and W Nethercot, "The breakdown of dielectrics under high voltage, with particular reference to thermal instability," Proceedings of the Physical Society, vol. 47, no. 5, p. 974, 1935.

[210] J. O'Dwyer, "Theory of dielectric breakdown in solids," Journal of The Electrochemical Society, vol. 116, no. 2, pp. 239$242,1969$.

[211] E. S. Anolick and G. R. Nelson, "Low field time dependent dielectric integrity," in Reliability Physics Symposium, 1979. 17th Annual, 1979, pp. 8-12.

[212] P. P. Budenstein, "On the mechanism of dielectric breakdown of solids," Electrical Insulation, IEEE Transactions on, vol. EI-15, no. 3, pp. 225-240, 1980.

[213] J. O'Dwyer, "Breakdown in solid dielectrics," Undetermined, IEEE Transactions on Electrical Insulation, vol. EI-17, no. 6, pp. $484-487,1982$.

[214] T. DiStefano, "Dielectric breakdown induced by sodium in MOS structures," Journal of Applied Physics, vol. 44, no. 1, pp. 527-528, 1973.

[215] J. McPherson, J.-Y. Kim, A. Shanware, and H. Mogul, "Thermochemical description of dielectric breakdown in high dielectric constant materials," Applied Physics Letters, vol. 82, no. 13, pp. 2121-2123, 2003.

[216] S. Blonkowski, "Filamentary model of dielectric breakdown," Journal of Applied Physics, vol. 107, no. 8, $084109,2010$.

[217] L. A. Dissado and P. J. J. Sweeney, "Physical model for breakdown structures in solid dielectrics," Phys. Rev. B, vol. 48, pp. $16261-16268,221993$.

[218] J. F. Scott, M. Azuma, C. A. P. de Araujo, L. D. McMillan, M. C. Scott, and T. Roberts, "Dielectric breakdown in high- $\epsilon$ films for ULSI DRAMs: II. barium-strontium titanate ceramics," Integrated Ferroelectrics, vol. 4, no. 1, pp. 61-84, 1994.

[219] H.-S. Kim, S. Campbell, and D. Gilmer, "Charge trapping and degradation in high-permittivity $\mathrm{TiO}_{2}$ dielectric films," Electron Device Letters, IEEE, vol. 18, no. 10, pp. 465-467, 1997.

[220] M. Danikas and G. Papaschinopoulos, "Thermal breakdown in solid dielectrics: a new approach," Journal of the Franklin Institute, vol. 335, no. 4, pp. $617-621,1998$.

[221] Y. Ye, S. Zhang, F. Dogan, E. Schamiloglu, J. Gaudet, P. Castro, M. Roybal, M. Joler, and C. Christodoulou, "Influence of nanocrystalline grain size on the breakdown strength of ceramic dielectrics," in Pulsed Power Conference, 2003. Digest of Technical Papers. PPC-2003. 14th IEEE International, vol. 1, 2003, 719-722 Vol.1.

[222] M. Roy, J. Nelson, R. MacCrone, L. Schadler, C. Reed, and R. Keefe, "Polymer nanocomposite dielectrics-the role of the interface," Dielectrics and Electrical Insulation, IEEE Transactions on, vol. 12, no. 4, pp. 629-643, 2005. 
[223] M. Touzin, D. Goeuriot, H.-J. Fitting, C. Guerret-Piécourt, D. Juvé, and D. Tréheux, "Relationships between dielectric breakdown resistance and charge transport in alumina materials - effects of the microstructure," Journal of the European Ceramic Society, vol. 27, no. 2-3, pp. $1193-1197,2007$.

[224] J. W. McPherson and H. C. Mogul, "Underlying physics of the thermochemical E model in describing low-field timedependent dielectric breakdown in $\mathrm{SiO}_{2}$ thin films," Journal of Applied Physics, vol. 84, no. 3, pp. 1513-1523, 1998.

[225] D. N. Nguyen, L. A. Emmert, D. Patel, C. S. Menoni, and W. Rudolph, "Transient phenomena in the dielectric breakdown of $\mathrm{HfO}_{2}$ optical films probed by ultrafast laser pulse pairs," Applied Physics Letters, vol. 97, no. 19, 191909, 2010.

[226] M. A. Wolak, A. S. Wan, J. S. Shirk, M. Mackey, A. Hiltner, and E. Baer, "Imaging the effect of dielectric breakdown in a multilayered polymer film," Journal of Applied Polymer Science, vol. 123, no. 4, pp. 2548-2557, 2012.

[227] K. Shubhakar, K. L. Pey, S. S. Kushvaha, S. J. O'Shea, N. Raghavan, M. Bosman, M. Kouda, K. Kakushima, and H. Iwai, "Grain boundary assisted degradation and breakdown study in cerium oxide gate dielectric using scanning tunneling microscopy," Applied Physics Letters, vol. 98, no. 7, 072902, 2011.

[228] K. Shubhakar, K. L. Pey, N. Raghavan, S. S. Kushvaha, M. Bosman, Z. Wang, and S. J. O'Shea, "Study of preferential localized degradation and breakdown of $\mathrm{HfO}_{2} / \mathrm{SiO}_{x}$ dielectric stacks at grain boundary sites of polycrystalline $\mathrm{HfO}_{2}$ dielectrics," Microelectronic Engineering, vol. 109, pp. 364 -369, 2013, Insulating Films on Semiconductors 2013.

[229] T. Nigam, A. Kerber, and P. Peumans, "Accurate model for time-dependent dielectric breakdown of high-k metal gate stacks," in Reliability Physics Symposium, 2009 IEEE International, 2009, pp. 523-530.

[230] L. Vandelli, A. Padovani, L. Larcher, G. Bersuker, J. Yum, and P. Pavan, "A physics-based model of the dielectric breakdown in hfo2 for statistical reliability prediction," in Reliability Physics Symposium (IRPS), 2011 IEEE International, 2011, GD.5.1-GD.5.4.

[231] G Bersuker, J Yum, L Vandelli, A Padovani, L Larcher, V Iglesias, M Porti, M Nafria, K McKenna, A Shluger, et al., "Grain boundary-driven leakage path formation in $\mathrm{HfO}_{2}$ dielectrics," Solid-State Electronics, vol. 65, pp. 146-150, 2011.

[232] A. J. Schmidt, R. Cheaito, and M. Chiesa, "A frequency-domain thermoreflectance method for the characterization of thermal properties," Review of Scientific Instruments, vol. 80, no. 9, 094901, pp. -, 2009.

[233] J. A. Malen, K. Baheti, T. Tao, Z. Yang, J. A. Hudgings, and A. Majumdar, "Optical measurement of thermal conductivity using fiber aligned frequency domain thermoreflectance," Journal of Heat Transfer, vol. 133, no. 8, 2011.

[234] Y. Wang, J. Y. Park, Y. K. Koh, and D. G. Cahill, "Thermoreflectance of metal transducers for time-domain thermoreflectance," Journal of Applied Physics, vol. 108, no. 4, 043507, 2010.

[235] J. Yang, C. Maragliano, and A. J. Schmidt, "Thermal property microscopy with frequency domain thermoreflectance," Review of Scientific Instruments, vol. 84, no. 10, 104904, p. 104904, 2013.

[236] B. Saleh and M. Teich, Fundamentals of Photonics, ser. Wiley Series in Pure and Applied Optics. Wiley, 2013.

[237] M. Ghotbi, M. Ebrahim-Zadeh, A. Majchrowski, E. Michalski, and I. V. Kityk, "High-average-power femtosecond pulse generation in the blue using $\mathrm{BiB}_{3} \mathrm{O}_{6}$," Opt. Lett., vol. 29, no. 21, pp. 2530-2532, 2004.

[238] M. Kaganov, I. Lifshitz, and L. Tanatarov, "Relaxation between electrons and the crystalline lattice," Soviet Physics JETP, vol. 4, no. 2, p. 174, 1957.

[239] S. Anisimov, B. Kapeliovich, and T. Perelman, "Electron emission from metal surfaces exposed to ultrashort laser pulses," Soviet Physics JETP, vol. 39, no. 2, p. 375, 1974.

[240] P. B. Allen, "Theory of thermal relaxation of electrons in metals," Phys. Rev. Lett., vol. 59, pp. 1460-1463, 13 1987.

[241] R. W. Schoenlein, W. Z. Lin, J. G. Fujimoto, and G. L. Eesley, "Femtosecond studies of nonequilibrium electronic processes in metals," Phys. Rev. Lett., vol. 58, pp. 1680-1683, 161987.

[242] H. E. Elsayed-Ali, T. B. Norris, M. A. Pessot, and G. A. Mourou, "Time-resolved observation of electron-phonon relaxation in copper," Phys. Rev. Lett., vol. 58, pp. 1212-1215, 121987. 
[243] R. H. M. Groeneveld, R. Sprik, and A. Lagendijk, "Effect of a nonthermal electron distribution on the electron-phonon energy relaxation process in noble metals," Phys. Rev. B, vol. 45, pp. 5079-5082, 91992.

[244] P. E. Hopkins, J. C. Duda, B. Kaehr, X. Wang Zhou, C.-Y. Peter Yang, and R. E. Jones, "Ultrafast and steady-state laser heating effects on electron relaxation and phonon coupling mechanisms in thin gold films," Applied Physics Letters, vol. 103, no. 21, 211910, 2013.

[245] A. Giri, J. T. Gaskins, B. F. Donovan, C. Szwejkowski, R. J. Warzoha, M. A. Rodriguez, J. Ihlefeld, and P. E. Hopkins, "Mechanisms of nonequilibrium electron-phonon coupling and thermal conductance at interfaces," Journal of Applied Physics, vol. 117, no. 10, 105105, p. 105 105, 2015.

[246] C. Thomsen, J. Strait, Z. Vardeny, H. J. Maris, J. Tauc, and J. J. Hauser, "Coherent phonon generation and detection by picosecond light pulses," Phys. Rev. Lett., vol. 53, pp. 989-992, 101984.

[247] C. Thomsen, H. T. Grahn, H. J. Maris, and J. Tauc, "Surface generation and detection of phonons by picosecond light pulses," Phys. Rev. B, vol. 34, pp. 4129-4138, 61986.

[248] H. Maris, "Picosecond ultrasonics," Scientific American, 1998.

[249] C. Thomsen, H. Maris, and J. Tauc, "Picosecond acoustics as a non-destructive tool for the characterization of very thin films," Thin Solid Films, vol. 154, no. 1-2, pp. $217-223,1987$.

[250] G. Tas and H. J. Maris, "Electron diffusion in metals studied by picosecond ultrasonics," Phys. Rev. B, vol. 49, pp. 15 04615 054, 211994.

[251] — - "Picosecond ultrasonic study of phonon reflection from solid-liquid interfaces," Phys. Rev. B, vol. 55, pp. 1852-1857, 31997.

[252] G. Tas, J. J. Loomis, H. J. Maris, A. A. Bailes, and L. E. Seiberling, "Picosecond ultrasonics study of the modification of interfacial bonding by ion implantation," Applied Physics Letters, vol. 72, no. 18, pp. 2235-2237, 1998.

[253] F. Decremps, M. Gauthier, S. Ayrinhac, L. Bove, L. Belliard, B. Perrin, M. Morand, G. L. Marchand, F. Bergame, and J. Philippe, "Picosecond acoustics method for measuring the thermodynamical properties of solids and liquids at high pressure and high temperature," Ultrasonics, no. 0, pp. -, 2014.

[254] G. T. Hohensee, W.-P. Hsieh, M. D. Losego, and D. G. Cahill, "Interpreting picosecond acoustics in the case of low interface stiffness," Review of Scientific Instruments, vol. 83, no. 11, 114902, pp. -, 2012.

[255] D. H. Hurley and K. L. Telschow, "Picosecond surface acoustic waves using a suboptical wavelength absorption grating," Phys. Rev. B, vol. 66, p. $153301,152002$.

[256] M. Nicoul, U. Shymanovich, A. Tarasevitch, D. von der Linde, and K. Sokolowski-Tinten, "Picosecond acoustic response of a laser-heated gold-film studied with time-resolved x-ray diffraction," Applied Physics Letters, vol. 98, no. 19, 191902, 2011.

[257] K. E. O'Hara, X. Hu, and D. G. Cahill, "Characterization of nanostructured metal films by picosecond acoustics and interferometry," Journal of Applied Physics, vol. 90, no. 9, pp. 4852-4858, 2001.

[258] M. Tomoda, O. Matsuda, O. Wright, and R. Voti, "Tomographic reconstruction of picosecond acoustic strain propagation," Applied Physics Letters, vol. 90, no. 4, p. 041 114, 2007.

[259] H. S. Carslaw and J. C. Jaeger, "Conduction of heat in solids," Oxford: Clarendon Press, 1959, 2nd ed., vol. $1,1959$.

[260] T. Bergman, A. Lavine, and F. Incropera, Fundamentals of Heat and Mass Transfer, 7th Edition. John Wiley \& Sons, Incorporated, 2011.

[261] R. Rosei and D. W. Lynch, "Thermomodulation spectra of Al, Au, and Cu," Phys. Rev. B, vol. 5, pp. 3883-3894, 10 1972.

[262] R. Bracewell, "The fourier transform and its applications," New York, vol. 5, 1965. 
[263] B. F. Donovan, C. J. Szwejkowski, J. C. Duda, R. Cheaito, J. T. Gaskins, C.-Y. Peter Yang, C. Constantin, R. E. Jones, and P. E. Hopkins, "Thermal boundary conductance across metal-gallium nitride interfaces from 80 to $450 \mathrm{~K}$," Applied Physics Letters, vol. 105, no. 20, 203502, p. 203 502, 2014.

[264] R. M. Costescu, M. A. Wall, and D. G. Cahill, "Thermal conductance of epitaxial interfaces," Phys. Rev. B, vol. 67, p. $054302,52003$.

[265] G. Jonker, "Some aspects of semiconducting barium titanate," Solid-State Electronics, vol. 7, no. 12, pp. 895 -903, 1964.

[266] H. Kishi, Y. Mizuno, and H. Chazono, "Base-metal electrode-multilayer ceramic capacitors: past, present and future perspectives," Japanese Journal of Applied Physics, vol. 42, no. Part 1, No. 1, pp. 1-15, 2003.

[267] S. M. Aygun, J. F. Ihlefeld, W. J. Borland, and J.-P. Maria, "Permittivity scaling in $\mathrm{Ba}_{1-x} \mathrm{Sr}_{x} \mathrm{TiO}_{3}$ thin films and ceramics," Journal of Applied Physics, vol. 109, no. 3, 034108, p. 034 108, 2011.

[268] A. Jezowski, J. Mucha, R. Pazik, and W. Strek, "Influence of crystallite size on the thermal conductivity in $\mathrm{BaTiO}_{3}$ nanoceramics," Applied Physics Letters, vol. 90, no. 11, 114104, p. 114 104, 2007.

[269] S. T. Davitadze, S. N. Kravchun, B. A. Strukov, B. M. Goltzman, V. V. Lemanov, and S. G. Shulman, "Specific heat and thermal conductivity of $\mathrm{BaTiO}_{3}$ polycrystalline thin films," Applied Physics Letters, vol. 80, no. 9, pp. 1631-1633, 2002.

[270] G. Chen, "Thermal conductivity and ballistic-phonon transport in the cross-plane direction of superlattices," Phys. Rev. $B$, vol. 57, pp. 14958-14973, Jun. 1998.

[271] Y. He, "Heat capacity, thermal conductivity, and thermal expansion of barium titanate-based ceramics," Thermochimica Acta, vol. 419, no. 1-2, pp. $135-141,2004$.

[272] C. T. Shelton, P. G. Kotula, G. L. Brennecka, P. G. Lam, K. E. Meyer, J.-P. Maria, B. J. Gibbons, and J. F. Ihlefeld, "Chemically homogeneous complex oxide thin films via improved substrate metallization," Advanced Functional Materials, vol. 22, no. 11, pp. 2295-2302, 2012.

[273] S. M. Aygun, P. Daniels, W. J. Borland, and J.-P. Maria, "In situ methods to explore microstructure evolution in chemically derived oxide thin films," Journal of Materials Research, vol. 25, pp. 427-436, 03 Mar. 2010.

[274] G. Koster, B. L. Kropman, G. J. H. M. Rijnders, D. H. A. Blank, and H. Rogalla, "Quasi-ideal strontium titanate crystal surfaces through formation of strontium hydroxide," Applied Physics Letters, vol. 73, pp. 2920-2922, 1998.

[275] A. R. Kortan, M. Hong, J. Kwo, J. P. Mannaerts, and N. Kopylov, "Structure of epitaxial $\mathrm{Gd}_{2} \mathrm{O}_{3}$ films grown on GaAs(100)," Phys. Rev. B, vol. 60, pp. 10 913-10918, 151999.

[276] H. Megaw, "Crystal structure of barium titanate," Nature, vol. 155, pp. 484-485, Apr. 1945.

[277] R. E. Newnham and Y. M. de Haan, "Refinement of the $\alpha \mathrm{Al}_{2} \mathrm{O}_{3}, \mathrm{Ti}_{2} \mathrm{O}_{3}, \mathrm{~V}_{2} \mathrm{O}_{3}$ and $\mathrm{Cr}_{2} \mathrm{O}_{3}$ structures," Zeitschrift für Kristallographie - Crystalline Materials, vol. 117, pp. 235-237, 1962.

[278] E112, Standard test methods for determining average grain size, ASTM, 2003.

[279] G. Furukawa, M. Reilly, and J. Gallagher, "Critical analysis of heat-capacity data and evaluation of thermodynamic properties of ruthenium, rhodium,palladium, iridium, and platinum from 0 to $300 \mathrm{~K}$," Journal of Physical and Chemical Reference Data, vol. 3, 1974.

[280] S. S. Todd and R. E. Lorenson, "Heat capacities at low temperatures and entropies at $298 \mathrm{~K}$ of metatitanates of barium and strontium," Journal of the American Chemical Society, vol. 74, no. 8, pp. 2043-2045, 1952.

[281] D. A. Ditmars, S. Ishihara, S. S. Chang, and G. Bernstein, "Enthalpy and heat-capacity standard reference material: synthetic sapphire $\left(\mathrm{a}_{-} \mathrm{Al}_{2} \mathrm{O}_{3}\right)$ from 10 to $2250 \mathrm{~K}$," Journal of Research of the National Bureau of Standards, vol. 87, no. 2, pp. 159-163, 1982.

[282] V. Pishchik, L. A. Lytvynov, and E. R. Dobrovinskaya, Sapphire - Material, Manufacturing, Applications, ser. Microand Opto-Electronic Materials, Structures, and Systems. ISBN 978-0-387-85695-7, 2009. 
[283] R. Yang and G. Chen, "Thermal conductivity modeling of periodic two-dimensional nanocomposites," Phys. Rev. B, vol. 69, p. $195316,192004$.

[284] P. E. Hopkins, P. M. Norris, and J. C. Duda, "Anharmonic phonon interactions at interfaces and contributions to thermal boundary conductance," Journal of Heat Transfer, vol. 133, no. 6, pp. 062 401-062 401, Mar. 2011.

[285] Z. Wang, J. E. Alaniz, W. Jang, J. E. Garay, and C. Dames, "Thermal conductivity of nanocrystalline silicon: importance of grain size and frequency-dependent mean free paths," Nano Letters, vol. 11, no. 6, pp. 2206-2213, 2011.

[286] A Maiti, G. Mahan, and S. Pantelides, "Dynamical simulations of nonequilibrium processes-heat flow and the kapitza resistance across grain boundaries," Solid State Communications, vol. 102, no. 7, pp. 517-521, 1997.

[287] P. Schelling, S. Phillpot, and P Keblinski, "Kapitza conductance and phonon scattering at grain boundaries by simulation," Journal of Applied Physics, vol. 95, no. 11, pp. 6082-6091, 2004.

[288] G. Shirane and A. Takeda, "Transition energy and volume change at three transitions in barium titanate," Journal of the Physical Society of Japan, vol. 7, no. 1, pp. 1-4, 1952.

[289] M. H. Frey and D. A. Payne, "Grain-size effect on structure and phase transformations for barium titanate," Phys. Rev. B, vol. 54, pp. 3158-3168, Aug. 1996.

[290] K. Kinoshita and A. Yamaji, "Grain-size effects on dielectric properties in barium titanate ceramics," Journal of Applied Physics, vol. 47, pp. 371-373, Jan. 1976.

[291] M. H. Frey, Z. Xu, P. Han, and D. A. Payne, "The role of interfaces on an apparent grain size effect on the dielectric properties for ferroelectric barium titanate ceramics," Ferroelectrics, vol. 206, no. 1, pp. 337-353, 1998.

[292] M. Asheghi, M. N. Touzelbaev, K. E. Goodson, Y. K. Leung, and S. S. Wong, "Temperature-dependent thermal conductivity of single-crystal silicon layers in soi substrates," Journal of Heat Transfer, vol. 120, no. 1, pp. 30-36, Feb. 1998.

[293] G. H. Zhu, H. Lee, Y. C. Lan, X. W. Wang, G. Joshi, D. Z. Wang, J. Yang, D. Vashaee, H. Guilbert, A. Pillitteri, M. S. Dresselhaus, G. Chen, and Z. F. Ren, "Increased phonon scattering by nanograins and point defects in nanostructured silicon with a low concentration of germanium," Phys. Rev. Lett., vol. 102, p. 196803, 192009.

[294] Y. Lee, S. Lee, and G. S. Hwang, "Effects of vacancy defects on thermal conductivity in crystalline silicon: a nonequilibrium molecular dynamics study," Phys. Rev. B, vol. 83, p. 125 202, 122011.

[295] J. F. Ihlefeld, B. M. Foley, D. A. Scrymgeour, J. R. Michael, B. B. McKenzie, D. L. Medlin, M. Wallace, S. TrolierMcKinstry, and P. E. Hopkins, "Room-temperature voltage tunable phonon thermal conductivity via reconfigurable interfaces in ferroelectric thin films," Nano Letters, vol. 15, no. 3, pp. 1791-1795, 2015, PMID: 25695423.

[296] B. Lokhande, P. Patil, and M. Uplane, "Studies on cadmium oxide sprayed thin films deposited through non-aqueous medium," Materials Chemistry and Physics, vol. 84, no. 2-3, pp. 238 -242, 2004.

[297] O. Madelung, U. Rössler, and M. Schulz, "Cadmium oxide (CdO) debye temperature, heat capacity, melting point, density," in II-VI and I-VII Compounds; Semimagnetic Compounds, ser. Landolt-Börnstein - Group III Condensed Matter, O. Madelung, U. Rössler, and M. Schulz, Eds., vol. 41B, Springer Berlin Heidelberg, 1999, pp. 1-3.

[298] M. Chase, "NIST-JANAF thermochemical tables," in Jouranl of Physical and Chemical Reference Data, Fourth, vol. 1, National Institute of Standards and Technology, 1998.

[299] L. N. A. Kandala, J. B. B. Rayappan, J. B. Gopalakrishnan, S. M. G. Manasai, and L. Nallathambi, "Effect of least variations in the lattice constant in the lattice dynamics of nanostructured CdO," Journal of Applied Sciences, vol. 12, 2012.

[300] Q. Lu, S. Wang, L. Li, J. Wang, S. Dai, W. Yu, and G. Fu, "Electrical and thermal transport properties of CdO ceramics," English, Science China Physics, Mechanics and Astronomy, pp. 1-5, 2014. 
[301] P. Kofstad, Nonstoichiometry, diffusion, and electrical conductivity in binary metal oxides. Wiley-Interscience New York, 1972 .

[302] J. Nowotny, T. Bak, M. K. Nowotny, and L. R. Sheppard, "Defect chemistry and electrical properties of titanium dioxide. 1. defect diagrams," The Journal of Physical Chemistry C, vol. 112, no. 2, pp. 590-601, 2008.

[303] A. Popov, A. Priezzhev, J Lademann, and R Myllyla, " $\mathrm{TiO}_{2}$ nanoparticles as an effective UV-B radiation skin-protective compound in sunscreens," Journal of Physics D: Applied Physics, vol. 38, no. 15, p. 2564, 2005.

[304] R. Blakey, "Evaluation of paint durability-natural and accelerated," progress in Organic Coatings, vol. 13, no. 5, pp. 279296, 1985.

[305] J. H. Braun, "Titanium dioxide's contribution to the durability of paint films," Progress in Organic Coatings, vol. 15, no. 3, pp. 249-260, 1987.

[306] Y. Kubota, C. Niwa, T. Ohnuma, Y. Ohko, T. Tatsuma, T. Mori, and A. Fujishima, "Protective effect of TiO 2 particles on UV light induced pyrimidine dimer formation," Journal of Photochemistry and Photobiology A: Chemistry, vol. 141, no. 2, pp. 225-230, 2001.

[307] O. Carp, C. L. Huisman, and A. Reller, "Photoinduced reactivity of titanium dioxide," Progress in solid state chemistry, vol. 32, no. 1, pp. 33-177, 2004.

[308] J. Yan, G. Wu, N. Guan, L. Li, Z. Li, and X. Cao, "Understanding the effect of surface/bulk defects on the photocatalytic activity of $\mathrm{TiO}_{2}$ : anatase versus rutile," Phys. Chem. Chem. Phys., vol. 15, pp. 10978-10988, 262013.

[309] J. Nowotny, M. A. Alim, T. Bak, M. A. Idris, M. Ionescu, K. Prince, M. Z. Sahdan, K. Sopian, M. A. Mat Teridi, and W. Sigmund, "Defect chemistry and defect engineering of $\mathrm{TiO}_{2}$-based semiconductors for solar energy conversion," Chem. Soc. Rev., vol. 44, pp. 8424-8442, 232015.

[310] G. K. Mor, K. Shankar, M. Paulose, O. K. Varghese, and C. A. Grimes, "Use of highly-ordered $\mathrm{TiO}_{2}$ nanotube arrays in dye-sensitized solar cells," Nano letters, vol. 6, no. 2, pp. 215-218, 2006.

[311] U. Bach, D Lupo, P Comte, J. Moser, F Weissortel, J Salbeck, H Spreitzer, and M Gratzel, "Solid-state dye-sensitized mesoporous $\mathrm{TiO}_{2}$ solar cells with high photon-to-electron conversion efficiencies," Nature, vol. 395, no. 6702, pp. 583-585, 1998.

[312] K. Zhu, N. R. Neale, A. Miedaner, and A. J. Frank, "Enhanced charge-collection efficiencies and light scattering in dye-sensitized solar cells using oriented $\mathrm{TiO}_{2}$ nanotubes arrays," Nano letters, vol. 7, no. 1, pp. 69-74, 2007.

[313] Z. Wu, D. Lee, M. F. Rubner, and R. E. Cohen, "Structural color in porous, superhydrophilic, and self-cleaning $\mathrm{SiO}_{2} / \mathrm{TiO}_{2}$ Bragg stacks," Small, vol. 3, no. 8, pp. 1445-1451, 2007.

[314] I. Pavlichenko, A. T. Exner, M. Guehl, P. Lugli, G. Scarpa, and B. V. Lotsch, "Humidity-enhanced thermally tunable $\mathrm{TiO}_{2} / \mathrm{SiO}_{2}$ bragg stacks," The Journal of Physical Chemistry C, vol. 116, no. 1, pp. 298-305, 2011.

[315] S. Y. Choi, M. Mamak, G. von Freymann, N. Chopra, and G. A. Ozin, "Mesoporous bragg stack color tunable sensors," Nano Letters, vol. 6, no. 11, pp. 2456-2461, 2006, PMID: 17090073.

[316] E. M. Golden, N. C. Giles, S. Yang, and L. E. Halliburton, "Interstitial silicon ions in rutile TiO 2 crystals," Phys. Rev. $B$, vol. 91, p. $134110,132015$.

[317] S. Lombardo, J. H. Stathis, B. P. Linder, K. L. Pey, F. Palumbo, and C. H. Tung, "Dielectric breakdown mechanisms in gate oxides," Journal of Applied Physics, vol. 98, no. 12, 121301, p. $121301,2005$.

[318] R. Waser, T. Baiatu, and K.-H. Härdtl, "Dc electrical degradation of perovskite-type titanates: I, ceramics," Journal of the American Ceramic Society, vol. 73, no. 6, pp. 1645-1653, 1990.

[319] - "Dc electrical degradation of perovskite-type titanates: II, single crystals," Journal of the American Ceramic Society, vol. 73, no. 6, pp. 1654-1662, 1990. 
[320] T. Baiatu, R. Waser, and K.-H. Härdtl, "Dc electrical degradation of perovskite-type titanates: III, a model of the mechanism," Journal of the American Ceramic Society, vol. 73, no. 6, pp. 1663-1673, 1990.

[321] J.-J. Wang, H.-B. Huang, T. J. Bayer, A. Moballegh, Y. Cao, A. Klein, E. C. Dickey, D. L. Irving, C. A. Randall, and L.-Q. Chen, "Defect chemistry and resistance degradation in Fe-doped $\mathrm{SrTiO}_{3}$ single crystal," Acta Materialia, vol. 108, pp. $229-240,2016$.

[322] M. Nowotny, T Bak, and J Nowotny, "Electrical properties and defect chemistry of $\mathrm{TiO}_{2}$ single crystal. III. equilibration kinetics and chemical diffusion," The Journal of Physical Chemistry B, vol. 110, no. 33, pp. 16 292-16 $301,2006$.

[323] Z.-G. Mei, Y. Wang, S.-L. Shang, and Z.-K. Liu, "First-principles study of lattice dynamics and thermodynamics of $\mathrm{TiO}_{2}$ polymorphs," Inorganic Chemistry, vol. 50, no. 15, pp. 6996-7003, 2011, PMID: 21714527.

[324] R. D. Shannon, "Revised effective ionic radii and systematic studies of interatomic distances in halides and chalcogenides," Acta Crystallographica Section A, vol. 32, no. 5, pp. 751-767, 1976.

[325] R. M. Hazen and L. W. Finger, "Bulk moduli and high-pressure crystal structures of rutile-type compounds," Journal of Physics and Chemistry of Solids, vol. 42, no. 3, pp. $143-151,1981$.

[326] D. G. Isaak, J. D. Carnes, O. L. Anderson, H. Cynn, and E. Hake, "Elasticity of $\mathrm{TiO}_{2}$ rutile to 1800K," English, Physics and Chemistry of Minerals, vol. 26, no. 1, pp. 31-43, 1998.

[327] B. F. Donovan, D. M. Long, A. Moballegh, E. C. Dickey, and P. E. Hopkins, "Impact of intrinsic point defect concentration on thermal transport in $\mathrm{TiO}_{2}$, , In Preparation, 2016.

[328] B. F. Donovan, W. A. Jensen, N. Liu, J. A. Floro, and P. E. Hopkins, "Strain induced reduction of silicon thermal conductivity with aluminum inclusions," In Preparation, 2016.

[329] L. Chen, B. F. Donovan, P. E. Hopkins, and S. J. Poon, "Length scale dependence of the thermal conductivity accumulation in nanograined Si-Ge alloys," In Preparation, 2016.

[330] B. F. Donovan, C. J. Szwejkowski, A. Giri, R. Cheaito, J. T. Gaskins, and P. E. Hopkins, "Contrast of macro-scale wetting and nano-scale thermal interactions between solids and fluorinated liquids," In Preparation, 2016.

[331] B. F. Donovan, A. Giri, J. T. Gaskins, and P. E. Hopkins, "Localized thin film dewetting and sourced and monitored via ultra-fast optics," Under Review, 2016.

[332] L. D. Zarzar, B. S. Swartzentruber, B. F. Donovan, P. E. Hopkins, and B. Kaehr, "Using laser-induced thermal voxels to pattern diverse materials at the solid-liquid interface," Under Review, 2016.

[333] A. Giri, J.-P. Niemelä, T. Tynell, J. T. Gaskins, B. F. Donovan, M. Karppinen, and P. E. Hopkins, "Heat-transport mechanisms in molecular building blocks of inorganic/organic hybrid superlattices," Phys. Rev. B, vol. 93, p. 115310,11 2016.

[334] R. Cheaito, J. T. Gaskins, M. E. Caplan, B. F. Donovan, B. M. Foley, A. Giri, J. C. Duda, C. J. Szwejkowski, C. Constantin, H. J. Brown-Shaklee, J. F. Ihlefeld, and P. E. Hopkins, "Thermal boundary conductance accumulation and interfacial phonon transmission: measurements and theory," Phys. Rev. B, vol. 91, p. $035432,32015$.

[335] C. J. Szwejkowski, N. C. Creange, K. Sun, A. Giri, B. F. Donovan, C. Constantin, and P. E. Hopkins, "Size effects in the thermal conductivity of gallium oxide $\left(\beta-\mathrm{G}_{2} 2 \mathrm{O}_{3}\right)$ films grown via open-atmosphere annealing of gallium nitride," Journal of Applied Physics, vol. 117, no. 8, 084308, p. $084308,2015$. 